PAULO OTÁVIO MOREIRA DOS SANTOS

\title{
ALGORITMOS EFICIENTES PARA ESTIMAÇÃO DE IMAGENS ACÚSTICAS
}

SÃO PAULO 

PAULO OTÁVIO MOREIRA DOS SANTOS

\title{
ALGORITMOS EFICIENTES PARA ESTIMAÇÃO DE IMAGENS ACÚSTICAS
}

\author{
Versão Corrigida
}

Dissertação apresentada à Escola Politécnica da Universidade de São Paulo para obtenção do Título de Mestre em Ciências. 



\title{
ALGORITMOS EFICIENTES PARA ESTIMAÇÃO DE IMAGENS ACÚSTICAS
}

\author{
Versão Corrigida
}

Dissertação apresentada à Escola Politécnica da Universidade de São Paulo para obtenção do Título de Mestre em Ciências.

Área de Concentração: Sistemas Eletrôni$\cos$

Orientador: Prof. Dr. Vítor Heloiz Nascimento 
Autorizo a reprodução e divulgação total ou parcial deste trabalho, por qualquer meio convencional ou eletrônico, para fins de estudo e pesquisa, desde que citada a fonte.

Este exemplar foi revisado e corrigido em relação à versão original, sob responsabilidade única do autor e com a anuência de seu orientador.

São Paulo, de de

Assinatura do autor:

Assinatura do orientador:

\section{Catalogação-na-publicação}

Santos, Paulo

Algoritmos eficientes para estimação de imagens acústicas / P. Santos -versão corr. -- São Paulo, 2021.

$142 \mathrm{p}$.

Dissertação (Mestrado) - Escola Politécnica da Universidade de São Paulo. Departamento de Engenharia de Sistemas Eletrônicos.

1.Processamento digital de sinais 2.Acústica 3.Transformadas rápidas I.Universidade de São Paulo. Escola Politécnica. Departamento de Engenharia de Sistemas Eletrônicos II.t. 


\section{Agradecimentos}

À minha família.

À Escola Politécnica da Universidade de São Paulo e a todos os seus professores e funcionários com os quais tive contato no decorrer das disciplinas.

Ao Prof. Dr. José Humberto Araújo Monteiro, ao Prof. Dr. Roger Fredy Larico Chavez e ao Prof. Dr. Omar Alexander Chura Vilcanqui que me incentivaram a ingressar no mestrado.

Ao orientador, Prof. Dr. Vítor Heloiz Nascimento, pela disposição em orientar o trabalho.

Aos membros da banca que se dispuseram gentilmente a participar.

À Fundação de Amparo à Pesquisa do Estado de São Paulo (FAPESP), através do processo nº 2017/22226-1, e à Coordenação de Aperfeiçoamento de Pessoal de Nível Superior (CAPES) por apoiar o trabalho. As opiniões, hipóteses e conclusões ou recomendações expressas neste material são de responsabilidade do autor e não necessariamente refletem a visão da FAPESP e da CAPES. 



\section{Resumo}

O problema de imageamento acústico consiste em mapear as direções e intensidades de fontes sonoras usando um arranjo de microfones. Vários métodos foram desenvolvidos para reduzir o custo computacional da estimação de imagens acústicas, a maioria deles é baseada na transformada de arranjo de Kronecker (KAT) ou a transformada rápida de Fourier para amostras que não são igualmente espaçadas (NFFT). Neste trabalho, mostramos como aplicar a KAT a métodos convencionais e também métodos mais avançados de otimização. A aplicação da KAT resulta numa redução significativa no uso de memória e no custo computacional para uma grande variedade de métodos. Estendemos o uso da KAT para novos algoritmos de otimização esparsa e desenvolvemos uma nova classe de algoritmos de homotopia para aplicações de imagens acústicas. A KAT também foi combinada com outros métodos eficientes para solução de problemas de mínimos quadrados, resultando em implementações eficientes de algoritmos bem establecidos como orthogonal matching pursuit (OMP). Finalmente, exploramos métodos encontrados na literatura que eram conceitualmente similares à KAT para chegar a uma nova maneira de estimar imagens acústicas usando produtos de Schur-Hadamard em conjunto com a KAT na solução eficiente de problemas de mínimos quadrados.

Palavras-chave: imagens acústicas. arranjos de microfones. beamforming. processamento de sinais. otimização esparsa. 



\section{Abstract}

The acoustic imaging problem consists of mapping the directions and intensities of sound sources using a microphone array. Several methods were developed for reducing the computational cost of acoustic image estimation, most of which are based either on the Kronecker Array Transform (KAT) or the non-equispaced fast Fourier transform (NFFT). In this work, we show how to apply the KAT to conventional methods and to more advanced optimization methods. The application of the KAT results in a significant reduction in memory usage and computational cost of a wide variety of methods. We extended the usage of the KAT to new sparse optimization algorithms and developed a new class of homotopy algorithms for acoustic image applications. The KAT was also combined with other efficient methods to solve least squares problems to arrive at very eficient implentations of well stabilished algorithms such as orthogonal matching pursuit (OMP). Finally, we explored methods found in the literatute that were conceptually similar to the KAT to arrive at a new way to estimate acoustic images using Schur-Hadamard products to solve least squares efficiently in conjunction with the KAT.

Keywords: acoustic images. microphone array. beamforming. signal processing. sparse optimization. 



\section{Lista de ilustrações}

Figura 1 - Arranjo com $N$ elementos. Imagem recriada com base em [1]. . . . . . 28

Figura 2 - Array com processamento linear. Imagem recriada com base em [1] . . 29

Figura 3 - Conformador de feixes atrasa-e-soma, comumente chamado de delayand-sum beamformer ou DAS. Imagem recriada com base em [1]. . . . 32

Figura 4 - Exemplo de imagem acústica gerada na análise de ruído de veículos. Imagem modificada de $[2] \ldots$. . . . . . . . . . . . . . . . . 44

Figura 5 - Exemplo de imagem acústica gerada na análise de reflexão de uma sala de concertos [3]. . . . . . . . . . . . . . . . . 45

Figura 6 - Sistema de coordenadas esféricas. . . . . . . . . . . . . . 46

Figura 7 - Amostragem do espaço $U[4] \ldots \ldots \ldots$. . . . . . . . . . . 47

Figura 8 - Geometrias de arranjos separáveis compostas de subarranjos lineares não uniformes e não redundantes. Cada ponto representa a posição de um microfone. . . . . . . . . . . . . . . . . . . . . . . . . . 67

Figura 9 - Aceleração média relativa do tempo de cálculo de X-KAT em relação a X-DAS para uma distribuição com três fontes pontuais e $N=64$. . . .

Figura 10 - Comparação dos tempos de cálculo de uma mesma imagem acústica utilizando CSM-DAS, CSM-KAT e NFFT baseada em CSM. As curvas correspondem a arranjos separáveis com $N=36$ (a) e $N=64$ (b) microfones. Cada ponto corresponde a uma média de 100 realizações de uma distribuição com três fontes na frequência de $10 \mathrm{kHz}$. . . . . . . . 74

Figura 11 - Comparação dos tempos de cálculo para quatro implementações diferentes de DAMAS: produto matricial; convolução bidimensional por PSF; convolução bidimensional acelerada por FFT; e acelerada por KAT. As curvas correspondem a arranjos separáveis com $N=36$ (a) e $N=64$ (b) microfones. Cada ponto corresponde a uma média de 100 realizações de uma distribuição com três fontes na frequência de $10 \mathrm{kHz}$ e foram realizadas 100 iterações do algoritmo para cada realização. A FFT bidimensional foi implementada com aplicações sequenciais de FFTs unidimensionais de tamanhos múltiplos de potências de dois. . .

Figura 12 - Memória ocupada pelas principais matrizes utilizadas nos métodos de estimação de imagens acústicas. O fato de algumas dessas matrizes serem hermitianas foi levado em consideração no cálculo da memória. 77 
Figura 13 - Comparação dos tempos de execução para MP-X considerando as acelerações conseguidas com aplicação da KAT. Todos as imagens foram calculadas na frequência de $11 \mathrm{kHz}$ com arranjos de geometria separável para uma distribuição de 10 fontes pontuais e cada ponto corresponde a uma média de 100 realizações. As rotinas foram implementadas em $\mathrm{C} / \mathrm{C}++$ e chamadas no Matlab na forma de funções MEX. . . . . . . . 84

Figura 14 - Comparação dos tempos de execução para OMP-X considerando as acelerações conseguidas com aplicação da KAT e de implementações recursivas baseadas nas decomposições de Cholesky e QR. Todos as imagens foram calculadas na frequência de $11 \mathrm{kHz}$ com arranjos de geometria separável e cada ponto corresponde a uma média de 100 realizações. As rotinas foram implementadas em $\mathrm{C} / \mathrm{C}++$ e chamadas no Matlab na forma de funções MEX. . . . . . . . . . . . . . . . . . . 88

Figura 15 - Porcentagem do tempo de total de cálculo devida a cada uma das tarefas principais realizadas pelo método de compressive beamforming para estimar uma imagem acústica em função da quantidade de iterações $K$. São mostrados dois casos com arranjos de geometria separável com $N=100$ e $N=64$. Todas as imagens possuíam uma distribuição com 30 fontes pontuais na frequência de $11 \mathrm{kHz}$ e $M_{x}=M_{y}=100$. A quantidade de iterações foi escolhida de forma que o algoritmo ainda não atingiu a convergência, executando todas as operações mencionadas a cada iteração . . . . . . . . . . . . . . . . . . . . . . . . . . . 991

Figura 16 - Porcentagem do tempo de total de cálculo devida a cada uma das tarefas principais realizadas pelo método de compressive beamforming para estimar uma imagem acústica em função do tamanho da imagem $M_{x}=M_{y}$. São mostrados dois casos com arranjos de geometria separável com $N=100$ e $N=64$. Todas as imagens possuíam uma distribuição com 30 fontes pontuais na frequência de $11 \mathrm{kHz}$ e $K=100$. A quantidade de iterações foi escolhida de forma que o algoritmo ainda não atingiu a convergência, executando todas as operações mencionadas a cada iteração. 92

Figura 17 - Comparação dos tempos de execução para compressive beamforming considerando as acelerações conseguidas com a aplicação das KATs direta e adjunta. Todas as imagens possuíam uma distribuição com 30 fontes pontuais na frequência de $11 \mathrm{kHz}$ e correspondem exatamente aos casos analisados nas Figuras 15 e 16. A opera de projeção foi implementada em $\mathrm{C}$ e chamada pelo Matlab [5]. total se refere ao tempo total de cálculo e prod mat ao tempo gasto com os produtos matriz-vetor. . . . . . . . . . . . . . . . . . . . 93 
Figura 18 - Porcentagem do tempo de total de cálculo devida a cada uma das tarefas principais realizadas pelo método de ajuste de covariância reescrito como um problema do tipo BPDN para estimar uma imagem acústica em função da quantidade de iterações $K$. São mostrados dois casos com arranjos de geometria separável com $N=100$ e $N=64$. Todas as imagens possuíam uma distribuição com 30 fontes pontuais na frequência de $11 \mathrm{kHz}$ e $M_{x}=M_{y}=100$. A quantidade de iterações foi escolhida de forma que o algoritmo ainda não atingiu a convergência, executando todas as operações mencionadas a cada iteração. . . . . . . . . . . . . . 94

Figura 19 - Porcentagem do tempo de total de cálculo devida a cada uma das tarefas principais realizadas pelo método de ajuste de covariância reescrito como um problema do tipo BPDN para estimar uma imagem acústica em função do tamanho da imagem $M_{x}=M_{y}$. São mostrados dois casos com arranjos de geometria separável com $N=100$ e $N=64$. Todas as imagens possuíam uma distribuição com 30 fontes pontuais na frequência de $11 \mathrm{kHz}$ e $M_{x}=M_{y}=100$. A quantidade de iterações foi escolhida de forma que o algoritmo ainda não atingiu a convergência, executando todas as operações mencionadas a cada iteração. . . . . . . . . . . . . .

Figura 20 - Comparação dos tempos de execução para ajuste de covariância reescrito como um problema BPDN considerando as acelerações conseguidas com a aplicação das KATs direta e adjunta. Todas as imagens possuíam uma distribuição com 30 fontes pontuais na frequência de $11 \mathrm{kHZ}$ e correspondem exatamente aos casos analisados nas Figuras 18 e 19. A operação de projeção foi implementada em C e chamada pelo Matlab [5]. total se refer ao tempo total de cálculo e prod mat ao tempo gasto com os produtos matriz-vetor. . . . . . . . . . . . . . . . . . . 96

Figura 21 - Comparação dos tempos de execução para TVAL3 considerando as acelerações conseguidas com a aplicação das CSM KATs direta e adjunta. Todas as imagens possuíam uma distribuição com 10 fontes pontuais na frequência de $11 \mathrm{kHz}$ e $K$ representa a quantidade de iterações. . . . 98

Figura 22 - Comparação dos tempos de execução para TVAL3 considerando as acelerações conseguidas com a aplicação das KATs direta e adjunta. Todas as imagens possuíam uma distribuição com 10 fontes pontuais na frequência de $11 \mathrm{kHz}$ e $K$ representa a quantidade de iterações. . . .

Figura 23 - Comparação dos tempos de execução para o algoritmo de homotopia $\ell_{0}$ para matriz decomponível em produtos de Kronecker considerando a aceleração conseguida com a KAT direta-adjunta. Todas as imagens foram calculadas na frequência de $10 \mathrm{kHz}$ e $\gamma=0,9 \ldots$. . . . . . . 108 
Figura 24 - Comparação de duas possíveis amostragens com o mesmo número de direções $M=120$ para KAT, uma restrita à região visível e outra espalhada por todo o espaço $U$, e uma possível amostragem para CZT. No caso da KAT, as direções foram distribuídas uniformemente nos retângulos $[-1,1]^{2}$ e $[-1 / \sqrt{2}, 1 / \sqrt{2}]^{2}$. No caso da CZT, as direções são distribuídas uniformemente no ângulo de elevação e uniformemente no cosseno do ângulo de azimute. Cada circunferência representa uma elevação e cada semi-reta partindo da origem representa um azimute. . 113

Figura 25 - Comparação do número de operações requeridas e memória em função da quantidade de direções pelos métodos XKAT e CZT com um mesmo arranjo de $N=400$ microfones arranjados numa grade $N_{x}=N_{y}=20$. Tomamos $M_{A}=M_{E}=M_{x}=M_{y}$ por simplicidade. . . . . . . . . 115

Figura 26 - Comparação do tempo de execução para OMP acelerado por SchurHadamard, as outras soluções baseadas em mínimos quadrados e a implementação rápida baseada em decomposição de Cholesky. Todas as rotinas foram escritas em $\mathrm{C} / \mathrm{C}++$ e chamadas no Matlab como funções MEX. São mostrados casos para dois arranjos separáveis, $N=49$ e $N=64$ e todas as imagens foram calculadas na frequência de $10 \mathrm{kHz} . ~ .121$

Figura 27 - Amostragem das direções de visada para $M_{x}=M_{y}=8 \ldots$. . . . . . 125

Figura 28 - Método de segmentação dos sinais de entrada de cada microfone em quadros de $K$ amostras cada. . . . . . . . . . . . . . . . . . 126

Figura 29 - Imagens acústicas geradas pelo simulador para um campo de $M=4096$ direções com três fontes pontuais em campo distante na frequência de $6 \mathrm{kHz}$. . . . . . . . . . . . . . . . . . . . . . . 128

Figura 30 - Imagens acústicas geradas pelo simulador para um campo de $M=1024$ direções com duas fontes pontuais em campo distante na frequência de $8 \mathrm{kHz}$. . . . . . . . . . . . . . . . . . . . 129

Figura 31 - Imagens acústicas geradas pelo simulador para um campo de $M=4096$ direções com quatro fontes pontuais em campo distante na frequência de $6 \mathrm{kHz}$ para diferentes números de iterações de DAMAS. . . . . . . . 130

Figura 32 - Imagens acústicas geradas pelo simulador para um campo de dimensões $M_{x}=M_{y}=32 \mathrm{com}$ duas fontes pontuais em campo distante na frequência de $11 \mathrm{kHz}$ para vários algoritmos diferentes de otimização esparsa implementados no sumulador. A posição das fontes é a mesma da Figura 30 porém uma das fontes possui aproximadamete a metade da potência da outra. . . . . . . . . . . . . . . . . . 131

Figura 33 - PSNR em função da frequência para as distribuições de fontes das Figuras 29 (a) e 30 (b). 


\section{Lista de tabelas}

Tabela 1 - Memória ocupada pelas principais matrizes complexas usadas no cálculo de imagens acústicas com precisão double. . . . . . . . . . . . . 76 



\section{Lista de abreviaturas e siglas}

KAT Kronecker array transform ou transformada de arranjo de Kronecker

LIT Linear e invariante no tempo

DAS Delay-and-sum beamformer ou beamformer convencional

NAH Nearfield acoustic holography ou holografia de campo próximo

PSF Point spread function ou função de resposta espacial

FFT Fast Fourier transform ou transformada rápida de Fourier

CSM Cross spectral matrix ou matriz espectral

MVDR Minimum variance distortionless response

MPDR Minimum power distortionless response

MMSE Minimum mean square error

SNR Signal-to-noise ratio ou relação sinal-ruído

DAMAS Deconvolution approach for the mapping of acoustic sources

CLEAN Algoritmo de deconvolução de imagens

NNFFT Nonequispaced in time and frequency fast Fourier transform

NFFT Nonequispaced fast Fourier transform

MP $\quad$ Matching pursuit

OMP Orthogonal matching pursuit

CB Compressive beamforming

MUSIC Multiple signal classification

ESPRIT Estimation of signal parameters via rotational invariant techniques

DOA Direction of arrival ou direção de chegada

BP Basis pursuit

BPDN Basis pursuit denoising 
CVX Pacote de software para otimização convexa

SPGL1 Pacote de software para resolução de problemas esparsos de mínimos quadrados

TV total variation ou variação total

TVAL3 TV minimization by augmented lagrangian and alternating direction algorithms

CD Coordinate descent ou coordenadas descendentes

DCD Dichotomous coordinate descent ou coordenadas descendentes dicotômicas

CZT $\quad$ chirp-Z transform ou transformada chirp-Z

AWGN Additive white Gaussian noise

DFT discrete Fourier transform ou transformada discreta de Fourier

MSE mean squared error ou erro quadrático médio

PSNR Peak signal-to-noise-ratio 


\section{Lista de símbolos}

$\mathbf{A}^{T}$

$\mathbf{A}^{*}$

$\mathbf{A}^{H}$

$\mathbf{A}^{\dagger}$

$\mathbf{A}_{:, k}$

$\mathbf{A}_{k,:}$

$\mathbf{A}_{\Lambda}$

I

$\operatorname{Re}\{\cdot\} \quad$ parte real

$\operatorname{Im}\{\cdot \quad$ parte imaginária

$E\{\cdot\} \quad$ valor esperado

$\operatorname{vec}\{\cdot\} \quad$ retorna um vetor coluna empilhando as colunas de uma matriz da esquerda para a direita

$\operatorname{vecd}\{\cdot\} \quad$ retorna um vetor coluna contendo a diagonal de uma matriz

$\operatorname{tr}\{\cdot \quad$ traço de uma matriz

$\otimes \quad$ operador do produto de Kronecker

$\odot$

$\circ$

$*$

$\mathbb{R}$

$\mathbb{C}$

$|x|$

$\|\mathbf{x}\|_{p}$

operador do produto de Khatri-Rao

operador do produto de Schur-Hadamard

operador de convolução

corpo dos números reais

corpo dos números complexos

valor absoluto de $x$

norma $\ell_{p}$ de um vetor $\mathbf{x}$ 
$\|\mathbf{x}\|_{2},\|\mathbf{x}\| \quad$ norma euclidiana de um vetor $\mathbf{x}$

$\|\mathbf{x}\|_{0} \quad$ pseudonorma $\ell_{0}$ de um vetor $\mathbf{x}$

$\|\mathbf{A}\|_{F} \quad$ norma de Frobenius da matriz A

$\|\mathbf{Y}\|_{B V} \quad$ variação total isotrópica de $\mathbf{Y}$

$\nabla_{x}, \nabla_{y} \quad$ operadores de primeira diferença

$\mid \mathcal{X} \quad$ cardinalidade do conjunto $\mathcal{X}$

$\mathcal{R}\{\cdot\}, \mathcal{N}\{\cdot\} \quad$ subespaços fundamentais 


\section{Sumário}

INTRODUÇÃo $\ldots \ldots \ldots \ldots \ldots \ldots \ldots$

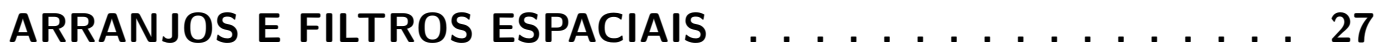

2.1 Resposta frequência-número de onda e padrões de radiação . . . . . 27

2.2 Considerações sobre largura de banda . . . . . . . . . . . . . 34

3 PROCESSOS ESPAÇOTEMPORAIS . . . . . . . . . . 37

3.1 Modelos snapshot no domínio da frequência . . . . . . . . . . 37

$3.2 \quad$ Modelo gaussiano . . . . . . . . . . . . . . . . 39

3.3 Representação em frequência-número de onda . . . . . . . . . . . 40

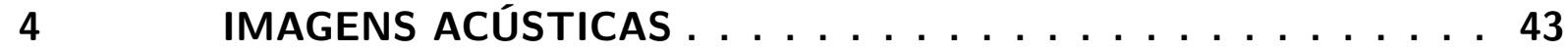

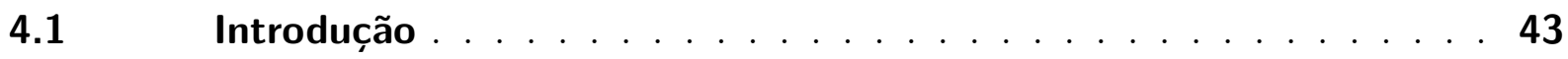

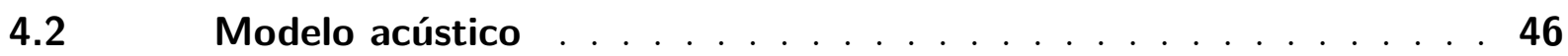

4.3 Métodos para imageamento acústico . . . . . . . . . . . . . 51

$4.3 .1 \quad$ Filtragem espacial . . . . . . . . . . . . . . . . . . . 51

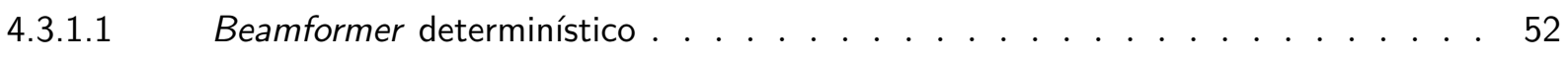

4.3.1.2 Beamformers ótimos . . . . . . . . . . . . . . . . 53

4.3.2 Métodos de deconvolução . . . . . . . . . . . . . . . . . 54

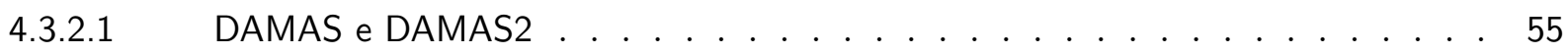

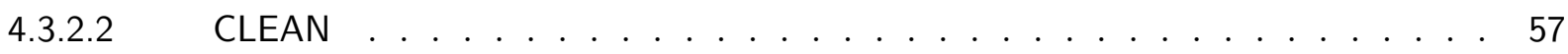

5 MÉTOdOS ACELERADOS PARA IMAGEAMENTO ACÚSTICO . 59

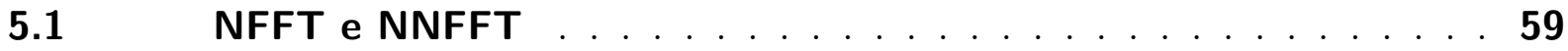

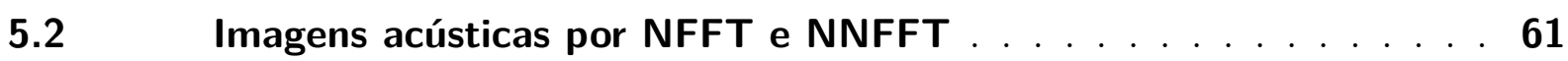

5.3 Transformada de arranjo de Kronecker . . . . . . . . . . . 64

5.3 .1 DAS acelerado por KAT . . . . . . . . . . . . . . 71

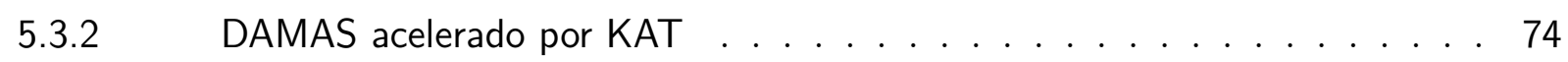

5.3.3 Implementações com a matriz A . . . . . . . . . . . . 75

6 IMAGEAMENTO POR OTIMIZAÇÃO REGULARIZADA . . . . . 79

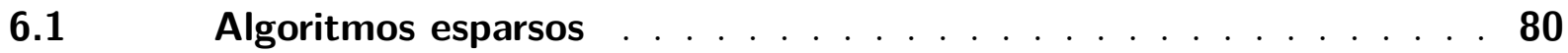

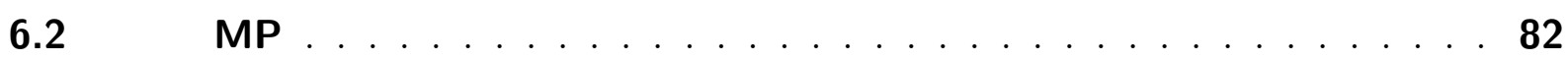

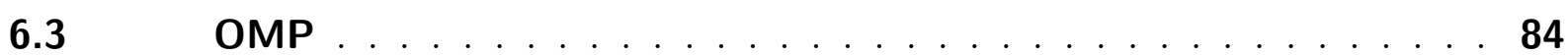

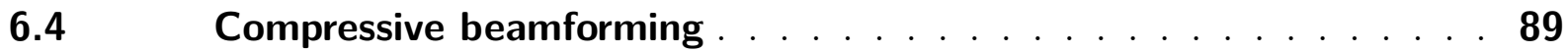

$6.5 \quad$ Ajuste de covariância com regularização $\ell_{1} \ldots \ldots \ldots 2$

6.6 Ajuste de covariância com regularização por variação total . . . . 96 
6.7 Ajuste do sinal com regularização por variação total . . . . . . . . . 98

6.8 Métodos de coordenadas descendentes . . . . . . . . . . . . 99

6.9 Homotopia para dicionários decomponíveis em produtos de Kronecker103

7 MÉTOdOS SIMILARES À KAT . . . . . . . . . . . . . . . 109

$7.1 \quad$ Transformada chirp $-Z \ldots \ldots$. . . . . . . . . . . . . . . . .

7.2 Imagens acústicas por Least Squares acelerado com produto de Schur-Hadamard . . . . . . . . . . . . . . . . . . . . 115

7.3 OMP acelerado por produto de Schur-Hadamard e KAT . . . . . . . 118

8 SIMULADOR PARA ESTIMAÇÃO DE IMAGENS ACÚSTICAS . . 123

8.1 Geometria do arranjo . . . . . . . . . . . . . . . . 123

$8.2 \quad$ Campo incidente e sinais nos microfones . . . . . . . . . . . . . . 123

8.3 Modelo de snapshots . . . . . . . . . . . . . . . . . 125

$8.4 \quad$ Geração das matrizes V e A . . . . . . . . . . . . . . . . . 127

8.5 Geração de imagens acústicas . . . . . . . . . . . . . . . . . . . 127

8.6 Comparação de qualidade das imagens obtidas . . . . . . . . . . . 131

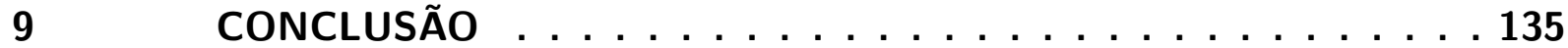

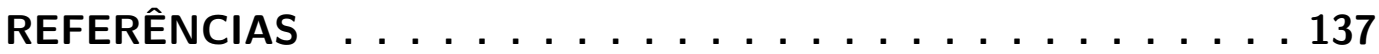




\section{Introdução}

Uma imagem acústica é gerada codificando-se os níveis de intensidade sonora em um mapa de cores, isto é, uma imagem na qual a escala de níveis de potência ou intensidade sonora é mapeada em uma escala de cores dos pixels.

O problema de imageamento acústico consiste no mapeamento de direções e intensidades de fontes sonoras utilizando um arranjo de microfones. O principal problema resolvido com o uso dessas imagens é a caracterização e detecção de fontes sonoras.

Com esse método, podem ser determinadas a presença e as direções de fontes em relação a um arranjo de microfones. Os níveis de intensidade podem ser determinados com o uso de filtragem espacial adequada à geometria do arranjo e às direções das fontes ou com uso de métodos de otimização regularizada. Também é possível estimar diretamente o sinal e até reconstruí-lo no domínio do tempo utilizando muitas vezes os mesmos algoritmos que estimam intensidade. O modelo de ondas planas é comumente utilizado.

Neste trabalho consideraremos algoritmos eficientes para a estimação de imagens acústicas visando análises de eficiência computacional e comparações entre diferentes tipos de algoritmos.

Existem três principais métodos de estimação de imagens acústicas: o mais simples computacionalmente e que apresenta resultados piores é beamforming, ou filtragem espacial; o segundo é o uso de deconvolução para melhorar os resultados obtidos por beamforming; o terceiro consiste em utilizar algoritmos mais avançados de otimização capazes de reconstruir a imagem de forma mais precisa, porém com custo computacional mais elevado.

O método convencional de beamforming tem a desvantagem de produzir imagens desfocadas e de baixa resolução, já que apresenta grandes lóbulos laterais, porém o custo computacional é baixo envolvendo apenas um produto matriz-vetor para obter a imagem completa. Podemos melhorar os resultados conseguidos com beamforming ao aplicar métodos iterativos de deconvolução resultando numa imagem menos desfocada, porém o custo computacional passa a ser um produto matriz-vetor por iteração. Por fim, os métodos avançados de otimização podem envolver o ajuste da matriz de covariância do sinal, otimização esparsa e métodos heurísticos; esses métodos possuem custo computacional geralmente mais elevado e acarretam em geral mais de um produto matriz-vetor por iteração.

O custo computacional elevado da estimação de imagens acústicas se deve principalmente às matrizes de grande dimensão envolvidas nos cálculos até mesmo para imagens de tamanho médio. A dimensão elevada das matrizes envolvidas também faz da estimação 
de imagens acústicas uma atividade que requer muita memória.

Neste contexto, exploramos o uso da transformada de arranjo de Kronecker (KAT) [6] que é capaz de reduzir o custo computacional de estimar imagens acústicas em algumas ordens de magnitude e o custo de memória em várias ordens de magnitude. A KAT é uma transformada rápida obtida ao escolher uma amostragem separável do espaço das possíveis direções de fontes sonoras e ao usar arranjos de microfones com geometria separável. Uma vez obedecidos os requisitos de geometria e amostragem, as matrizes envolvidas passam a possuir uma estrutura que pode ser decomposta em produtos de Kronecker, permitindo a derivação e aplicação de várias versões da KAT.

Neste trabalho, mostramos como derivar e aplicar a KAT a vários algoritmos para estimação de imagens acústicas e veremos que ela se aplica a todas as três classes de algoritmos mencionadas. Estendemos o uso da KAT para novos algoritmos de estimação esparsa para os quais ela ainda não havia sido aplicada e a combinamos com outros métodos acelerados resultando em implementações eficientes de métodos heurísticos. Em especial, desenvolvemos um novo algoritmo de homotopia $\ell_{0}$ que leva em consideração a decomposição de Kronecker das matrizes envolvidas. Também mostramos outras transformadas rápidas que podem ser aplicadas nos casos em que a geometria ou a amostragem não são compatíveis com a KAT.

Parte deste trabalho foi publicada em [7], em que apresentamos uma nova forma de estimar imagens baseada em soluções de mínimos quadrados aceleradas por produtos de Schur-Hadamard aplicados em conjunto com a KAT. Além disso, combinamos a KAT e o método de Schur-Hadamard para acelerar ainda mais a estimação de imagens por orthogonal matching pursuit (OMP).

Também exploramos métodos similares à KAT encontrados na literatura para fins de comparação e derivação de novas classes de algoritmos eficientes para estimação de imagens acústicas.

Por fim, descrevemos o simulador de imagens acústicas desenvolvido em ambiente Matlab que foi utilizado para implementar a estimação de imagens acústicas.

A organização do texto é dada a seguir. O capítulo 2 apresenta conceitos básicos de arranjos e define filtragem espacial. O capítulo 3 traz uma introdução aos processos aleatórios espaçotemporais que permite abordar os casos em que os sinais do arranjo estão sujeitos ao ruído. O capítulo 4 apresenta a definição de imagem acústica e trata dos principais métodos de beamforming e deconvolução. No capítulo 5, mostramos as transformadas rápidas para o cálculo de imagens acústicas e discutimos a redução do custo computacional e do impacto na memória. O capítulo 6 trata de vários algoritmos de otimização esparsa, como eles podem se beneficiar da KAT, e apresenta algumas aplicações da KAT a algoritmos novos. Também apresentamos uma nova classe de algoritmos de 
homotopia com baixo custo de memória que se valem da KAT e da estrutura decomponível das matrizes envolvidas. O capítulo 7 trata dos métodos conceitualmente simulares à KAT e resulta na combinação da KAT com um novo método acelerado em problemas de estimação da intensidade sonora para casos esparsos. O capítulo 8 trata da implementação do simulador de imagens acústicas que foi desenvolvido. 



\section{Arranjos e filtros espaciais}

Um arranjo de sensores consiste em um grupo de sensores distribuídos de determinada forma em uma região do espaço. Estes sensores são utilizados para medir campos de ondas propagantes, que podem ser eletromagnéticas, sísmicas ou acústicas. Arranjos de sensores encontram aplicações em qualquer campo que envolva estimação de ondas. Algumas aplicações são: aquisição de imagens médicas por ultrassom; detecção e rastreamento de fenômenos meteorológicos; controle de tráfego aéreo; redes de telefonia celular; medição de ondas sísmicas; detecção e rastreamento de ondas subaquáticas; medições de campo acústico; aquisição de voz; interligação de rádio-telescópios; entre outras aplicações [8].

Os objetivos do processamento de sinais com arranjos de sensores são: melhorar a razão sinal-ruído; caracterizar o campo propagante determinando o número de fontes, a localização destas fontes e as formas de onda emitidas; rastrear fontes conforme elas se movem no espaço [9].

Assume-se que múltiplos sinais se propaguem por alguma região do espaço e que também exista interferência (ruído) em alguma outra região do espaço, sendo que a região de propagação dos sinais e a de existência de ruído podem se interceptar. Sob tais condições, um arranjo é utilizado para filtrar um campo no tempo e no espaço. No domínio da frequência, este processo de filtragem consiste em combinar as saídas dos sensores do arranjo aplicando ganhos complexos que amplificam ou atenuam os sinais de acordo com sua dependência espacial. Em geral é necessário amplificar sinais de direções específicas e rejeitar ruído presente em outras direções.

Existem três aspectos que determinam a performance dos arranjos como filtros espaciais: a sua geometria; a escolha dos ganhos complexos de cada sensor; e a resposta espacial de cada sensor do arranjo. A geometria do arranjo geralmente é determinada por questões físicas, de fabricação e de projeto [1], e a escolha dos ganhos complexos determina a característica de filtragem espacial do arranjo para uma geometria específica.

Neste capítulo serão expostos os conceitos básicos de arranjos tendo como base a exposição de [1]. Os conceitos mais importantes abordados serão o vetor diretor do arranjo, que representa sua característica espacial, e o conceito de filtragem espacial.

\subsection{Resposta frequência-número de onda e padrões de radiação}

Seja um arranjo composto de um conjunto de $N$ sensores isotrópicos localizados nas posições $\mathbf{p}_{0}, \mathbf{p}_{1}, \ldots, \mathbf{p}_{N-1}$. A geometria do arranjo é arbitrária e as coordenadas $\mathbf{p}_{n}$ se

encontram no $\mathbb{R}^{3}$, como mostrado na Figura 1. Assumindo um campo de onda incidindo 


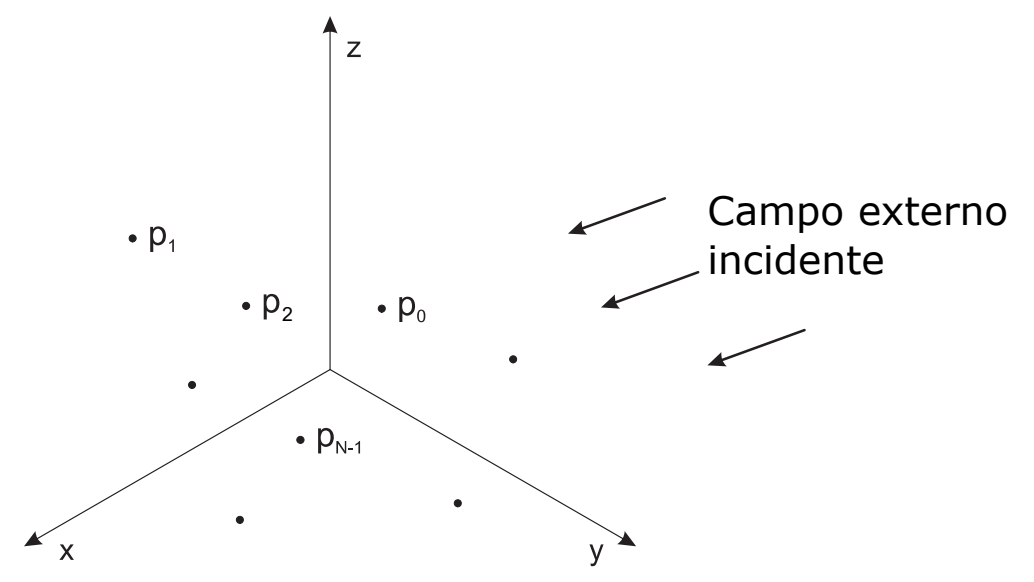

Figura 1 - Arranjo com $N$ elementos. Imagem recriada com base em [1].

no arranjo, temos um conjunto de sinais nas posições dos sensores dado por

$$
\mathbf{f}(t, \mathbf{p})=\left[\begin{array}{c}
f\left(t, \mathbf{p}_{0}\right) \\
f\left(t, \mathbf{p}_{1}\right) \\
\vdots \\
f\left(t, \mathbf{p}_{N-1}\right)
\end{array}\right]
$$

A saída de cada sensor é processada por um filtro linear invariante no tempo $\left(\mathrm{LIT}^{1}\right)$ com resposta ao impulso $h_{n}(\tau)$ e todas as saídas são somadas para se obter $y(t)$, a saída do arranjo. Esse processo é ilustrado pelo diagrama de blocos da Figura 2.

Da teoria dos sistemas LIT e do diagrama da Figura 2, sabe-se que a saída $y(t)$ pode ser escrita na forma de uma convolução dada por

$$
y(t)=\sum_{n=0}^{N-1} \int_{-\infty}^{\infty} h_{n}(t-\tau) f\left(\tau, \mathbf{p}_{n}\right) d \tau .
$$

Essa última expressão pode ser reescrita como

$$
y(t)=\int_{-\infty}^{\infty}\left\{h_{0}(t-\tau) f\left(\tau, \mathbf{p}_{0}\right)+h_{1}(t-\tau) f\left(\tau, \mathbf{p}_{1}\right)+\ldots h_{N-1}(t-\tau) f\left(\tau, \mathbf{p}_{N-1}\right)\right\} d \tau
$$

que pode ser simplificada para

$$
y(t)=\int_{-\infty}^{\infty}\left[\begin{array}{llll}
h_{0}(t-\tau) & h_{1}(t-\tau) & \cdots & h_{N-1}(t-\tau)
\end{array}\right]\left[\begin{array}{c}
f\left(\tau, \mathbf{p}_{0}\right) \\
f\left(\tau, \mathbf{p}_{1}\right) \\
\vdots \\
f\left(\tau, \mathbf{p}_{N-1}\right)
\end{array}\right] d \tau
$$

Resultando na notação vetorial para a saída $y(t)$ dada por

$$
y(t)=\int_{-\infty}^{\infty} \mathbf{h}^{T}(t-\tau) \mathbf{f}(\tau, \mathbf{p}) d \tau
$$

\footnotetext{
1 Em inglês, LTI ou linear time-invariant.
} 


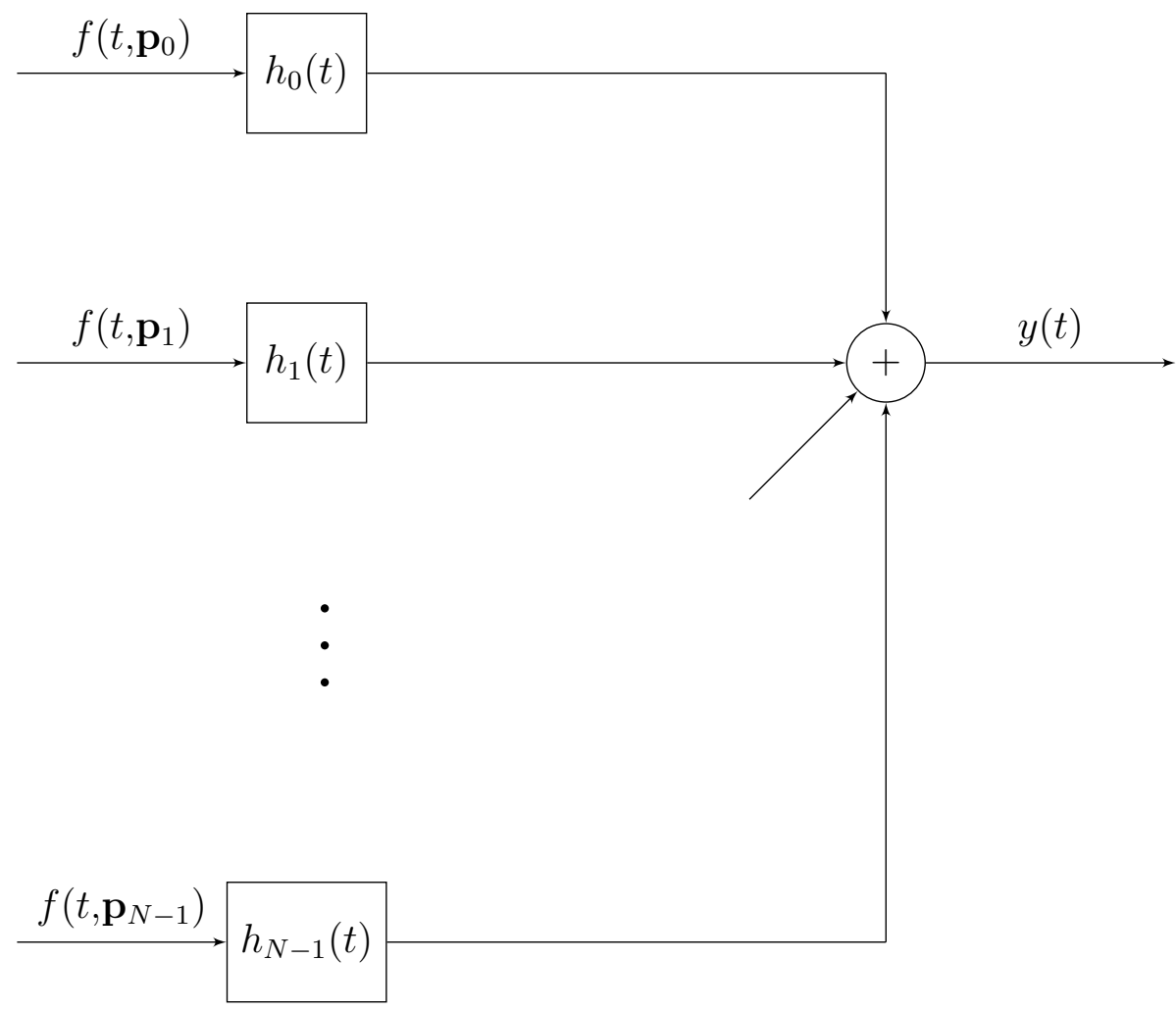

Figura 2 - Array com processamento linear. Imagem recriada com base em [1].

em que

$$
\mathbf{h}(t)=\left[\begin{array}{c}
h_{0}(t) \\
h_{1}(t) \\
\vdots \\
h_{N-1}(t)
\end{array}\right]
$$

é o vetor de respostas a impulso do sistema para cada entrada correspondente aos $N$ sensores do arranjo.

Também da teoria de sistemas LIT, sabemos que podemos tomar a transformada de Fourier da equação (2.5) para obter uma representação do sistema no domínio da frequência:

$$
\begin{aligned}
y(\omega) & =\int_{-\infty}^{\infty} y(t) e^{-j \omega t} d t \\
& =\int_{-\infty}^{\infty}\left[\mathbf{h}^{T}(t) * \mathbf{f}(t, \mathbf{p})\right] e^{-j \omega t} d t \\
& =\mathbf{h}^{T}(\omega) \mathbf{f}(\omega, \mathbf{p}),
\end{aligned}
$$

em que $\mathbf{h}(\omega)$ e $\mathbf{f}(\omega, \mathbf{p})$ são as transformadas de Fourier de $\mathbf{h}(t)$ e $\mathbf{f}(t, \mathbf{p})$, respectivamente.

Consideremos agora o caso mostrado na Figura 1, porém com o campo de onda externo incidente modelado por uma onda plana se propagando na direção a com frequência $\omega$. 
Seja $f(t)$ o sinal que é recebido na origem do sistema de coordenadas adotado. Podemos escrever

$$
\mathbf{f}(t, \mathbf{p})=\left[\begin{array}{c}
f\left(t-\tau_{0}\right) \\
f\left(t-\tau_{1}\right) \\
\vdots \\
f\left(t-\tau_{N-1}\right)
\end{array}\right]
$$

em que

$$
\tau_{n}=\frac{\mathbf{a}^{T} \mathbf{p}_{n}}{c}
$$

$c$ é a velocidade de propagação no meio e a pode ser expresso em coordenadas esféricas como

$$
\mathbf{a}=\left[\begin{array}{c}
-\sin \theta \cos \phi \\
-\sin \theta \sin \phi \\
-\cos \theta
\end{array}\right]
$$

O sinal de menos indica a direção de a, que é contrária à direção de visada do arranjo

$$
\mathbf{u}=-\mathbf{a}
$$

e podemos escrever

$$
\tau_{n}=-\frac{\mathbf{u}^{T} \mathbf{p}_{n}}{c}
$$

Note que a é um vetor unitário que corresponde à direção de propagação da onda. Já u possui a mesma direção de a, porém com sentido oposto, e corresponde à direção de visada do arranjo. Note também que o produto interno da direção de propagação da onda com a posição de um microfone, $\mathbf{a}^{T} \mathbf{p}_{n}$, representa a distância a ser percorrida pela onda para ir da origem até o sensor $\mathbf{p}_{n}$ e, se esse valor for negativo, significa que o sinal num sensor específico está adiantado em relação ao sinal captado na origem. Dividir essa distância pela velocidade de propagação $c$ nos dá o atraso $\tau_{n}$.

Tomando a transformada de Fourier da equação (2.8), teremos

$$
\mathbf{f}(\omega)=\int_{-\infty}^{\infty} e^{-j \omega t} \mathbf{f}(t, \mathbf{p}) d t=\left[\begin{array}{c}
f(\omega) e^{-j \omega \tau_{0}} \\
f(\omega) e^{-j \omega \tau_{1}} \\
\vdots \\
f(\omega) e^{-j \omega \tau_{N-1}}
\end{array}\right]
$$

em que

$$
\omega \tau_{n}=\frac{\omega}{c} \mathbf{a}^{T} \mathbf{p}_{n}
$$

Para ondas planas se propagando em meio homogêneo, o vetor de número de onda é definido como

$$
\mathbf{k}=\frac{\omega}{c} \mathbf{a}=\frac{2 \pi}{\lambda} \mathbf{a}=-\frac{2 \pi}{\lambda} \mathbf{u}
$$


em que $\lambda$ denota o comprimento de onda. A solução da equação da onda obriga que ${ }^{2}$

$$
\|\mathbf{k}\|_{2}=\frac{\omega}{c}=\frac{2 \pi}{\lambda}
$$

Portanto, apenas a direção de $\mathbf{k}$, e não sua magnitude, varia para uma determinada frequência $\omega$. Substituir a equação (2.15) na equação (2.9) leva a

$$
\omega \tau_{n}=\mathbf{k}^{T} \mathbf{p}_{n}
$$

Também podemos expressar $\omega \tau_{n}$ em função de $\mathbf{u}$ fazendo

$$
\mathbf{k}=-\frac{\omega}{c} \mathbf{u}
$$

o que resulta em

$$
\omega \tau_{n}=-\frac{\omega}{c} \mathbf{u}^{T} \mathbf{p}_{n}
$$

Se definirmos

$$
\mathbf{v}_{\mathbf{k}}(\mathbf{k})=\left[\begin{array}{c}
e^{-j \mathbf{k}^{T} \mathbf{p}_{0}} \\
e^{-j \mathbf{k}^{T} \mathbf{p}_{1}} \\
\vdots \\
e^{-j \mathbf{k}^{T} \mathbf{p}_{N-1}}
\end{array}\right]
$$

então podemos escrever

$$
\begin{aligned}
\mathbf{f}(\omega) & =\left[\begin{array}{c}
f(\omega) e^{-j \omega \tau_{0}} \\
f(\omega) e^{-j \omega \tau_{1}} \\
\vdots \\
f(\omega) e^{-j \omega \tau_{N-1}}
\end{array}\right]=\left[\begin{array}{c}
f(\omega) e^{-j \mathbf{k}^{T} \mathbf{p}_{0}} \\
f(\omega) e^{-j \mathbf{k}^{T} \mathbf{p}_{1}} \\
\vdots \\
f(\omega) e^{-j \mathbf{k}^{T} \mathbf{p}_{N-1}}
\end{array}\right] \\
= & f(\omega) \mathbf{v}_{\mathbf{k}}(\mathbf{k}) .
\end{aligned}
$$

$\mathrm{O}$ vetor $\mathbf{v}_{\mathbf{k}}(\mathbf{k})$ é chamado de vetor de resposta ou vetor diretor ${ }^{3}$. Note que esse desenvolvimento permite escrever $\mathbf{f}(\omega)$ como o produto do sinal escalar da onda medido na origem por um vetor que carrega as características espacias de propagação da onda e de posicionamento do arranjo.

Agora, analisemos o que acontece caso as entradas de cada sensor sejam atrasadas ou adiantadas de forma que estejam todas alinhadas no tempo e posteriormente somadas, incluindo a aplicação de um fator de normalização $1 / N$. Fazendo isso, obtém-se $f(t)$ como saída, conforme mostrado na Figura 3. Neste caso, a resposta a impulso de cada atraso no tempo é

$$
h_{n}(t)=\frac{1}{N} \delta\left(t+\tau_{n}\right)
$$

$\mathrm{e}$

$$
y(t)=f(t)
$$




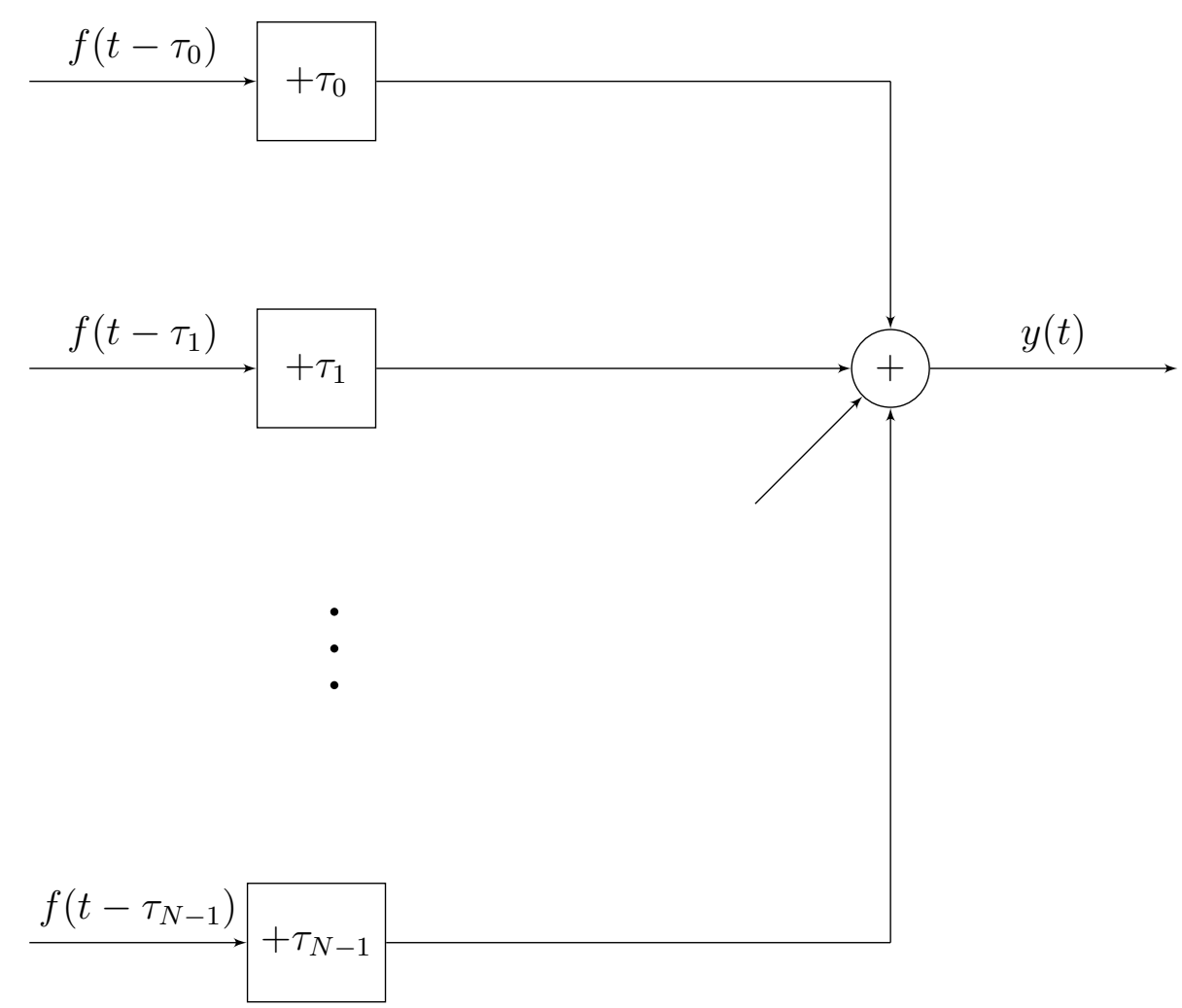

Figura 3 - Conformador de feixes atrasa-e-soma, comumente chamado de delay-and-sum beamformer ou DAS. Imagem recriada com base em [1].

Essa operação é conhecida como delay-and-sum beamformer (DAS) ou beamformer ${ }^{4}$ convencional. Para que a operação mostrada na Figura 3 seja realizável, são aplicados atrasos a todos os sinais de forma a alinhá-los no tempo, e não adiantamentos como mostrado no diagrama.

Podemos expressar esta operação em forma vetorial como

$$
\mathbf{h}^{T}(\omega)=\frac{1}{N} \mathbf{v}_{\mathbf{k}}\left(\mathbf{k}_{s}\right)
$$

em que $\mathbf{k}_{s}$ é o vetor de número de onda da onda plana de interesse. O beamformer DAS é o mais simples e tem um papel fundamental no desenvolvimento deste trabalho, além de servir como ponto de partida para algoritmos mais eficientes para estimação de imagens acústicas que serão discutidos no capítulo 4.

Se considerarmos o problema mais geral de encontrar a resposta do arranjo para um campo de entrada arbitrário $f(t, \mathbf{p})$, que pode possuir várias fontes, então podemos encontrá-la por convolução utilizando a teoria de sistemas LIT estendida para o caso de várias variáveis. No entanto, é mais fácil encontrar uma solução para uma única onda plana em função de sua frequência temporal $\omega$ e seu vetor de número de onda $\mathbf{k}$ e utilizar

$2 \quad\|\cdot\|_{2}$ denota a norma euclidiana.

3 A nomenclatura mais comumente encontrada na literatura para vetor diretor é array manifold vector.

4 Conformador ou formador de feixe, em português. Neste trabalho optou-se por utilizar a nomenclatura em inglês para o termo beamformer. 
os resultados da análise de Fourier para representar a resposta de um sistema LIT quando as entradas são exponenciais complexas.

Aplicando ainda a teoria de sistemas LIT, porém estendida para sinais no tempo e no espaço, pode-se analisar a resposta do sistema em termos da superposição de funções exponenciais complexas que formam uma base de um espaço vetorial. As funções da base são ondas da forma

$$
f_{n}\left(t, \mathbf{p}_{n}\right)=\exp \left[j\left(\omega t-\mathbf{k}^{T} \mathbf{p}_{n}\right)\right]
$$

$\mathrm{Ou}$

$$
f_{n}\left(t, \mathbf{p}_{n}\right)=e^{j \omega t} e^{-j \mathbf{k}^{T} \mathbf{p}_{n}}
$$

portanto

$$
\mathbf{f}(t, \mathbf{p})=e^{j \omega t} \mathbf{v}_{\mathbf{k}}(\mathbf{k})
$$

ou seja, estamos considerando apenas uma onda com frequência temporal $\omega$ e com características de propagação definidas por seu vetor $\mathbf{k}$.

Da teoria de sistemas LIT, sabemos que a resposta para uma exponencial complexa também é uma exponencial complexa de mesma frequência [10]. Sendo assim, a resposta do processador mostrado na Figura 2 a uma onda plana é

$$
y(t, \mathbf{k})=\mathbf{h}^{T}(\omega) \mathbf{v}_{\mathbf{k}}(\mathbf{k}) e^{j \omega t} .
$$

Escrever a última equação no domínio da frequência leva a

$$
y(\omega, \mathbf{k})=\mathbf{h}^{T}(\omega) \mathbf{v}_{\mathbf{k}}(\mathbf{k}) .
$$

O processamento temporal e espacial realizado pelo arranjo é completamente descrito pela equação anterior. Definimos

$$
\Upsilon(\omega, \mathbf{k}) \triangleq \mathbf{h}^{T}(\omega) \mathbf{v}_{\mathbf{k}}(\mathbf{k})
$$

que chamamos função de resposta em frequência-número de onda. Tal função descreve o ganho de um arranjo para uma onda plana de entrada e é análoga a uma resposta em frequência de um sistema LIT. O primeiro termo $\mathbf{h}(\omega)$ corresponde a um filtro LIT aplicado aos sinais captados pelo arranjo e o segundo termo $\mathbf{v}_{\mathbf{k}}(\mathbf{k})$ é o vetor diretor do arranjo.

O padrão de radiação ${ }^{5}$ de um arranjo é definido para ondas planas se propagando em um meio homogêneo, isto é, a condição de restrição de k é dada pela equação (2.16), que deriva diretamente da equação da onda. Ele é obtido avaliando a resposta frequêncianúmero de onda em relação à direção de visada ou de propagação, parametrizada por

$$
\mathbf{k}(\theta, \phi)=\frac{2 \pi}{\lambda} \mathbf{a}(\theta, \phi)=-\frac{2 \pi}{\lambda} \mathbf{u}(\theta, \phi)
$$

\footnotetext{
5 Em inglês, beam pattern.
} 
em que $\mathbf{a}(\theta, \phi)$ é a direção de propagação dada em coordenadas esféricas em função dos ângulos $\theta$ e $\phi$ definidos como na Figura 6. Em suma, o padrão de radiação é a função de frequência-número de onda avaliada em uma esfera de raio $2 \pi / \lambda$ :

$$
B(\omega, \theta, \phi)=\left.\Upsilon(\omega, \mathbf{k})\right|_{\mathbf{k}=\frac{2 \pi}{\lambda} \mathbf{a}(\theta, \phi)}
$$

\subsection{Considerações sobre largura de banda}

Consideremos o caso de sinais passa-faixa, isto é,

$$
f\left(t, \mathbf{p}_{n}\right)=\sqrt{2} \operatorname{Re}\left\{\tilde{f}\left(t, \mathbf{p}_{n}\right) e^{j \omega_{c} t}\right\}
$$

em que $\omega_{c}$ é a frequência da portadora e $\tilde{f}\left(t, \mathbf{p}_{n}\right)$ é o envelope complexo. Assumindo o envelope complexo limitado em banda à região

$$
\left|\omega-\omega_{c}\right| \leq \pi B_{s}
$$

em que $\pi B_{s}$ é uma constante especificando a largura de banda máxima do envelope complexo, teremos, para a onda plana descrita pela equação (2.8),

$$
f\left(t, \mathbf{p}_{n}\right)=\sqrt{2} \operatorname{Re}\left\{\tilde{f}\left(t-\tau_{n}\right) e^{j \omega_{c}\left(t-\tau_{n}\right)}\right\} .
$$

Consideremos agora o tempo de propagação de um sinal através do arranjo. Defina $\Delta T_{n m}(\mathbf{u})$ como o tempo de propagação entre o $n$-ésimo e o $m$-ésimo elementos do arranjo para uma onda plana que se propaga na direção u. Então,

$$
\Delta T_{\max } \triangleq \max _{n, m=0,1, \ldots, N-1}\left\{\Delta T_{n m}(\mathbf{u})\right\}
$$

é o máximo tempo de propagação entre quaisquer dois elementos do arranjo.

Agora, assumindo que a origem esteja localizada no centro de gravidade do arranjo, isto é,

$$
\sum_{n=0}^{N-1} \mathbf{p}_{n}=0
$$

Então, todos os $\tau_{n}$ satisfazem

$$
\tau_{n} \leq \Delta T_{\max }
$$

ou seja, o atraso entre qualquer sinal nos pontos $\mathbf{p}_{n}$ e a origem para uma onda plana propagando na direção u é menor que o atraso máximo entre quaisquer dois elementos do arranjo.

Vamos assumir que a largura de banda do envelope é pequena o suficiente para valer que

$$
\tilde{f}\left(t-\tau_{n}\right) \approx \tilde{f}(t), n=0,1, \ldots, N-1
$$


Para que a aproximação anterior seja válida, é necessário que

$$
B_{s} \Delta T_{\max } \ll 1 \text {. }
$$

Os sinais que obedecem à restrição da equação (2.40) são ditos de banda estreita. Utilizando esta condição, chega-se a

$$
f\left(t, \mathbf{p}_{n}\right)=\sqrt{2} \operatorname{Re}\left\{\tilde{f}(t) e^{-j \omega_{c} \tau_{n}} e^{j \omega_{c} t}\right\} .
$$

Quando esta restrição é verdadeira, o atraso pode ser aproximado por uma simples mudança de fase. Esta implementação é conhecida como phased array.

Este desenvolvimento é importante no contexto de processamento de sinais de banda larga, já que os beamformers de banda larga geralmente são implementados como bancos de beamformers de banda estreita [8]. 



\section{Processos espaçotemporais}

Neste capítulo será apresentada a linguagem utilizada para caracterizar processos aleatórios espaçotemporais. Esta abordagem estatística permite abordar os casos em que os sinais recebidos pelo arranjo são constituídos de sinais desejados, sinais causando interferência e ruído. O material apresentado nessa seção se baseia principalmente em $[1,8,11]$.

\subsection{Modelos snapshot no domínio da frequência}

Muitas aplicações práticas implementam a operação de beamforming no domínio da frequência, usualmente envolvendo três etapas: converter o vetor de entrada dos sensores para o domínio da frequência; processar o vetor de entrada a fim de obter uma função escalar no domínio da frequência; e converter a função escalar da frequência obtida no passo anterior para o domínio do tempo. O objetivo da primeira parte, ou do primeiro processador $^{1}$, é gerar um conjunto de vetores complexos chamados snapshots, ou blocos.

Para poder gerar esses vetores, primeiro divide-se o intervalo total de observação $T$ em $K$ pequenos intervalos disjuntos de comprimento $\Delta T$. Estes intervalos são indexados em $k$ e dados por

$$
(k-1) \Delta T \leq t<k \Delta T, k=1,2, \ldots, k, \ldots, K
$$

Agora, é necessário estabelecer um critério de escolha de $\Delta T$ que seja apropriado. A primeira restrição em $\Delta T$ é ser significativamente maior que o tempo de propagação de um sinal através do arranjo. Definindo

$$
\Delta T_{\text {max }} \triangleq \max _{d=1, \ldots, D}\left\{\max _{i, j}\left\{\Delta T_{i j}^{(d)}\right\}\right\},
$$

em que $\Delta T_{i j}^{(d)}$ denota o tempo de propagação entre o $i$-ésimo e o $j$-ésimo elementos para o $d$-ésimo sinal. Então, restringe-se

$$
\Delta T \gg \Delta T_{\max }
$$

de forma que o $\Delta T$ apropriado deve ser muito maior que o máximo tempo de propagação entre dois elementos quaisquer do arranjo para o sinal de entrada que demora mais para se propagar dentre todos os sinais incidentes no arranjo.

A segunda restrição a ser imposta está relacionada com com a largura de banda dos sinais de entrada e o formato de seus espectros. Assumindo um sinal de entrada

1 Neste trabalho, o termo processador deve ser entendido como um bloco de processamento de sinais ou uma etapa do processamento de sinal de algum sistema, e não no sentido usual de processador de computadores. 
como um processo estocástico passa-faixa com largura de banda $B_{s}$ de média zero com espectro de formato retangular e centrado na frequência $\omega_{c}$, denotando o sinal como $\mathbf{x}(t)$ e considerando o intervalo $[0, \Delta T)$, procedendo como [12] e [13], define-se

$$
\mathbf{x}_{\Delta T}\left(\omega_{m}\right)=\frac{1}{\sqrt{\Delta T}} \int_{0}^{\Delta T} \mathbf{x}(t) e^{-j\left(\omega_{c}+m \omega_{\Delta}\right) t} d t . m=-\frac{M-1}{2}, \ldots, 0, \ldots, \frac{M-1}{2},
$$

Na equação anterior, $\mathbf{x}_{\Delta T}\left(\omega_{m}\right)$ denota um vetor de dimensão $N$ de coeficientes de uma série de Fourier na $m$-ésima frequência $\omega_{m}=\omega_{c}+m \omega_{\Delta}$ para cada elemento do arranjo e $\mathbf{x}(t)$ é o vetor de funções amostradas do processo estocástico em cada ponto no qual existe um elemento do arranjo. A resolução dessa transformada de Fourier é

$$
\omega_{\Delta}=\frac{2 \pi}{\Delta T} \text {. }
$$

Estamos interessados em estabelecer um critério para escolha de $\Delta T$ de forma que os coeficientes de Fourier em diferentes frequências e diferentes snapshots sejam descorrelacionados. Para isso, precisamos de uma expressão da matriz de covariância entre $\mathbf{x}_{\Delta T}\left(\omega_{m_{1}}\right)$ e $\mathbf{x}_{\Delta T}\left(\omega_{m_{2}}\right)$, isto é,

$$
\mathbf{S}_{\mathbf{x}_{\Delta T}}\left(m_{1}, m_{2}\right) \triangleq E\left[\mathbf{x}_{\Delta T}\left(\omega_{m_{1}}\right) \mathbf{x}_{\Delta T}^{H}\left(\omega_{m_{2}}\right)\right]
$$

em que $E[\cdot]$ denota o valor esperado e $[\cdot]^{H}$ denota a conjugada transposta. Então, aplicando a equação (3.4), temos

$$
\mathbf{S}_{\mathbf{x}_{\Delta T}}\left(m_{1}, m_{2}\right)=\frac{1}{\Delta T} \int_{0}^{\Delta T} \int_{0}^{\Delta T} \mathbf{R}_{\mathbf{x}}(t-u) e^{-j \omega_{c}(t-u)} e^{-j m_{1} \omega_{\Delta} t+j m_{2} \omega_{\Delta} u} d t d u
$$

em que $\mathbf{R}_{\mathbf{x}}$ denota autocorrelação.

Reescrevendo a equação anterior, chega-se a

$$
\mathbf{S}_{\mathbf{x}_{\Delta T}}\left(m_{1}, m_{2}\right)=\frac{1}{\Delta T} \int_{-\infty}^{\infty} \int_{0}^{\Delta T} \int_{0}^{\Delta T} \frac{1}{2 \pi} \mathbf{S}_{\mathbf{x}}(\omega) e^{j\left(\omega-\omega_{c}\right)(t-u)} e^{-j m_{1} \omega_{\Delta} t} e^{j m_{2} \omega_{\Delta} u} d t d u d \omega,
$$

em que $\mathbf{S}_{\mathbf{x}}$ é a matriz espectral do processo. Definindo $\omega_{L}=\omega-\omega_{c}$, fazendo $m_{1}=m_{2}=m$ e depois de alguma manipulação algébrica teremos que

$$
\mathbf{S}_{\mathbf{x}_{\Delta T}}(m, m)=\int_{-\infty}^{\infty} \mathbf{S}_{\mathbf{X}}\left(\omega_{L}+\omega_{c}\right)\left[\frac{\operatorname{sinc}^{2}\left\{\pi\left(\frac{\omega_{L}}{\omega_{\Delta}}-m\right)\right\}}{\omega_{\Delta}}\right] d \omega_{L}
$$

No limite, com $\Delta T \rightarrow \infty$, o termo entre colchetes da equação (3.9) tende a um impulso, portanto

$$
\lim _{\Delta T \rightarrow \infty} \mathbf{S}_{\mathbf{x}_{\Delta T}}(m, m)=\mathbf{S}_{\mathbf{x}}\left(\omega_{c}+m \omega_{\Delta}\right) .
$$

Este resultado é o Teorema de Wiener-Khinchin². Pode-se mostrar que o resultado é válido para todos os termos da matriz, tanto os da diagonal quanto os termos fora da diagonal $[1,12]$.

2 O teorema afirma que a densidade espectral de potência de um processo estocástico estacionário é dada pela transformada de Fourier da função de autocorrelação. Veja [11] para mais detalhes. 
Baseado na equação (3.10) sabe-se que $\mathbf{S}_{\mathbf{x}}$, a matriz espectral do processo, pode ser aproximada arbitrariamente por $\mathbf{S}_{\mathbf{x}_{\Delta T}}$. Porém, é necessário um critério de escolha de $\Delta T$ para que a aproximação seja válida. O autor de [1] mostra que um produto $B_{S} \cdot \Delta T \geq 16$ é uma condição suficiente para que a aproximação seja precisa.

Existe ainda outra questão a ser analisada. Assumimos um espectro de formato retangular, isto é, um espectro plano. Porém, na prática os espectros de interesse não são planos. Os autores de [12] mostraram que basta que o espectro seja constante num intervalo $\pm 2 \omega_{\Delta}$ a $\pm 3 \omega_{\Delta}$ para que a aproximação ainda seja boa.

Portanto, cada pequeno intervalo deve ser suficientemente longo para garantir um espectro aproximadamente constante na vizinhança de cada frequência central $\omega_{c}$. E, nestas condições, os autores de [12] mostraram que os snapshots correspondentes a frequências diferentes serão aproximadamente descorrelacionados fazendo com que possam ser processados isoladamente, isto é, que o modelo seja válido.

\subsection{Modelo gaussiano}

A literatura de processamento de sinais com arranjos costuma utilizar vetores aleatórios gaussianos para modelar sinais, interferências e ruído. Se supusermos que o sinal $\mathbf{x}(t)$ na entrada do arranjo é um processo estocástico gaussiano real, pode ser mostrado que o snapshot $\mathbf{x}_{\Delta T}\left(\omega_{m}\right)$ é um funcional linear de $\mathbf{x}(t)$, portanto suas amostras também são variáveis aleatórias conjuntamente gaussianas. Da discussão da seção anterior, decorre que $\mathbf{x}_{\Delta T}\left(\omega_{m}\right)$ é um vetor de variáveis aleatórias circulares complexas [14, 15].

Sejam

$$
\begin{aligned}
\mathbf{m}_{\Delta T}\left(\omega_{m}, k\right) & =E\left\{\mathbf{x}_{\Delta T}\left(\omega_{m}, k\right)\right\} \\
\mathbf{S}_{\mathbf{x}, \Delta T}\left(\omega_{m}, k\right) & =E\left\{\mathbf{x}_{\Delta T}\left(\omega_{m}, k\right) \mathbf{x}_{\Delta T}^{H}\left(\omega_{m}, k\right)\right\} \\
\mathbf{K}_{\mathbf{x}, \Delta T}\left(\omega_{m}, k\right) & =E\left\{\left[\mathbf{x}_{\Delta T}\left(\omega_{m}, k\right)-\mathbf{m}_{\Delta T}\left(\omega_{m}, k\right)\right]\left[\mathbf{x}_{\Delta T}^{H}\left(\omega_{m}, k\right)-\mathbf{m}_{\Delta T}^{H}\left(\omega_{m}, k\right)\right]\right\}
\end{aligned}
$$

a média, correlação e covariância do vetor aleatório $\mathbf{x}_{\Delta T}\left(\omega_{m}, k\right)$. Sua distribuição, para média zero e média diferente de zero, é, então, dada por [1]

$$
\begin{aligned}
& p_{\mathbf{x}, \Delta T}=\frac{1}{\pi^{N}\left|\mathbf{S}_{\mathbf{x}, \Delta T}\right|} \exp \left\{-\mathbf{x}_{\Delta T}^{H} \mathbf{S}_{\mathbf{x}, \Delta T}^{-1} \mathbf{x}_{\Delta T}\right\} \\
& p_{\mathbf{x}, \Delta T}=\frac{1}{\pi^{N}\left|\mathbf{K}_{\mathbf{x}, \Delta T}\right|} \exp \left\{-\left[\mathbf{x}_{\Delta T}-\mathbf{m}_{\Delta T}\right]^{H} \mathbf{K}_{\mathbf{x}, \Delta T}^{-1}\left[\mathbf{x}_{\Delta T}-\mathbf{m}_{\Delta T}\right]\right\} .
\end{aligned}
$$

A partir dessas definições, pode-se estabelecer o modelo de ondas planas na linguagem de processo aleatórios. Suponhamos que a saída do arranjo seja

$$
\begin{aligned}
\mathbf{x}(t) & =\mathbf{x}_{s}(t)+\boldsymbol{\eta}(t), \\
\mathbf{x}\left(\omega_{m}\right) & =\mathbf{x}_{s}\left(\omega_{m}\right)+\boldsymbol{\eta}\left(\omega_{m}\right),
\end{aligned}
$$


em que $\mathbf{x}_{s}(t)$ representa um sinal determinístico e $\boldsymbol{\eta}(t)$ representa ruído modelado como um processo gaussiano. Supondo que o sinal foi gerado por uma fonte de ondas planas com espectro $f_{s}\left(\omega_{m}\right)$ na direção $\mathbf{k}_{s}$, temos

$$
\begin{aligned}
\mathbf{x}\left(\omega_{m}\right) & =\mathbf{v}\left(\omega_{m}, \mathbf{k}_{s}\right) f_{s}\left(\omega_{m}\right), \\
p_{\mathbf{x}}(\mathbf{x}) & =\frac{1}{\pi^{N}\left|\mathbf{S}_{n}\right|} \exp \left\{-\left[\mathbf{x}-\mathbf{v}\left(\mathbf{k}_{s}\right) f_{s}\right]^{H} \mathbf{S}_{n}^{-1}\left[\mathbf{x}-\mathbf{v}\left(\mathbf{k}_{s}\right) f_{s}\right]\right\},
\end{aligned}
$$

em que $\mathbf{S}_{n}\left(\omega_{m}\right)$ é a matriz espectral de potência de $\boldsymbol{\eta}(t)$.

\subsection{Representação em frequência-número de onda}

Seja um processo aleatório escalar e complexo $f(t, \mathbf{p})$ definido no espaço e no tempo com $t \in \mathbb{R}$ e $\mathbf{p} \in \mathbb{R}^{3}$. Definimos

$$
\begin{aligned}
m_{f}(t, \mathbf{p}) & =E\{f(t, \mathbf{p})\}, \\
K_{f}\left(t_{1}, t_{2}, \mathbf{p}_{1}, \mathbf{p}_{2}\right) & =E\left\{\left[f\left(t_{1}, \mathbf{p}_{1}\right)-m_{f}\left(t_{1}, \mathbf{p}_{1}\right)\right]\left[f^{*}\left(t_{2}, \mathbf{p}_{2}\right)-m_{f}^{*}\left(t_{2}, \mathbf{p}_{2}\right)\right]\right\},
\end{aligned}
$$

que são, respectivamente, a média e a correlação espaçotemporal do processo. Admitindo que vale

$$
K_{f}\left(t_{1}, t_{2}, \mathbf{p}_{1}, \mathbf{p}_{2}\right)=K_{f}\left(t_{1}-t_{2}, \mathbf{p}_{1}-\mathbf{p}_{2}\right)=K_{f}(\tau, \Delta \mathbf{p}),
$$

isto é, que o processo é estacionário no tempo e homogêneo no espaço, poderemos usar a transformada de Fourier para definir funções nas variáveis $\tau$ e $\Delta \mathbf{p}$ que levam aos domínios de $\omega$ e k. Perceba que, de acordo com o capítulo 2, isso equivale a uma representação no domínio frequência-número de onda.

Como esse problema possui variáveis nos domínios temporais e espaciais, podemos aplicar a Transformada de Fourier nos dois domínios separadamente, em ambos ao mesmo tempo ou um seguido do outro. Definimos então as funções espectro em frequência-correlação espacial $S_{f}(\omega, \Delta \mathbf{p})$, correlação temporal-espectro em número de onda $F_{f}(\tau, \mathbf{k})$ e espectro em frequência-número de onda $P_{f}(\omega, \mathbf{k})$ como

$$
\begin{aligned}
S_{f}(\omega, \Delta \mathbf{p}) & =\int_{-\infty}^{\infty} K_{f}(\tau, \Delta \mathbf{p}) e^{-j \omega \tau} d \tau, \\
F_{f}(\tau, \mathbf{k}) & =\int_{\mathbb{R}^{3}} K_{f}(\tau, \Delta \mathbf{p}) e^{j \mathbf{k}^{T} \Delta \mathbf{p}} d \Delta \mathbf{p}, \\
P_{f}(\omega, \mathbf{k}) & =\int_{\mathbb{R}^{3}} S_{f}(\omega, \Delta \mathbf{p}) e^{j \mathbf{k}^{T} \Delta \mathbf{p}} d \Delta \mathbf{p} .
\end{aligned}
$$

As inversas dessas equações são obtidas através da aplicação análoga da transformada inversa de Fourier:

$$
\begin{aligned}
& K_{f}(\tau, \Delta \mathbf{p})=\frac{1}{2 \pi} \int_{-\infty}^{\infty} S_{f}(\omega, \Delta \mathbf{p}) e^{j \omega \tau} d \omega, \\
& K_{f}(\tau, \Delta \mathbf{p})=\frac{1}{(2 \pi)^{3}} \int_{\mathbb{R}^{3}} F_{f}(\tau, \mathbf{k}) e^{-j \mathbf{k}^{T} \Delta \mathbf{p}} d \mathbf{k} \\
& S_{f}(\omega, \Delta \mathbf{p})=\frac{1}{(2 \pi)^{3}} \int_{\mathbb{R}^{3}} P_{f}(\omega, \mathbf{k}) e^{-j \mathbf{k}^{T} \Delta \mathbf{p}} d \mathbf{k} .
\end{aligned}
$$


Essas definições, segundo [8], permitem generalizar o teorema de Wiener-Khinchin e mostrar que o espectro em frequência-número de onda tomado em retângulos disjuntos ${ }^{3}$ é descorrelacionado, provando que os processos estacionários no tempo e homogêneos no espaço podem ser representados como uma superposição de ondas planas descorrelacionadas. Os desenvolvimentos do capítulo 4, como veremos mais adiante, utilizarão esse fato para modelagem das fontes acústicas.

3 Na verdade, na generalização de retângulos formados pelo produto cartesiano de quatro intervalos reais, formando um volume no espaço $\mathbb{R}^{4}$. Veja [8] para o desenvolvimento da prova. 



\section{Imagens acústicas}

Este capítulo introduz a definição de imagem acústica como uma versão discretizada do espectro frequência-número de onda de um processo aleatório. Depois o modelo de sinal é apresentado, do qual obtemos as equações principais usadas nos algoritmos de cálculo de imagens acústicas. Depois a vetorização das equações é discutida. Por fim, discutimos dois métodos de estimação de imagens acústicas: beamforming e deconvolução. Para cada método, alguns dos algoritmos mais comuns são discutidos. A maior parte do material apresentado aqui é baseado em $[8,16,17,18]$.

\subsection{Introdução}

Seja um processo aleatório espaçotemporal estacionário no tempo e homogêneo no espaço. De acordo com [8], sua correlação espaçotemporal $K(\tau, \Delta \mathbf{p})$, seu espectro frequência-correlação espacial $S(\omega, \Delta \mathbf{p})$ e seu espectro frequência-número de onda $P(\omega, \mathbf{k})$ são dados pelas equações

$$
\begin{aligned}
S(\omega, \Delta \mathbf{p}) & =\int_{-\infty}^{\infty} K(\tau, \Delta \mathbf{p}) e^{-j \omega \tau} d \tau, \\
P(\omega, \mathbf{k}) & =\int_{\mathbb{R}^{3}} S(\omega, \Delta \mathbf{p}) e^{j \mathbf{k}^{T} \Delta \mathbf{p}} d \Delta \mathbf{p}, \\
K(\tau, \Delta \mathbf{p}) & =\frac{1}{2 \pi} \int_{-\infty}^{\infty} S(\omega, \Delta \mathbf{p}) e^{j \omega \tau} d \omega, \\
S(\omega, \Delta \mathbf{p}) & =\frac{1}{(2 \pi)^{3}} \int_{\mathbb{R}^{3}} P(\omega, \mathbf{k}) e^{-j \mathbf{k}^{T} \Delta \mathbf{p}} d \mathbf{k} .
\end{aligned}
$$

Uma versão discretizada de $P(\omega, \mathbf{k})$, para $\omega$ fixo e $\mathbf{k}$ amostrado sobre uma região de interesse, de um processo com correlação espaçotemporal $K(\tau, \Delta \mathbf{p})$, é chamada imagem acústica. No entanto, $\mathbf{k}$ deve ser parametrizado em apenas duas coordenadas para que a imagem acústica seja bidimensional [8]. Um exemplo de imagem acústica com aplicação industrial é mostrado na Figura 4.

De forma mais rigorosa, $P(\omega, \mathbf{k})$ representa a densidade espectral de potência do processo, portanto é real e não negativa. Como os processos estacionários e homogêneos podem ser representados como uma superposição de ondas planas descorrelacionadas em frequência e direção de propagação [1], então $P(\omega, \mathbf{k})$ é proporcional à potência por frequência e por unidade de ângulo sólido que deve ser emitida por uma distribuição de fontes localizadas no campo distante. Portanto, uma discretização de $P(\omega, \mathbf{k})$ equivale a uma discretização da distribuição de fontes de um processo estacionário espaçotemporal supondo válida a hipótese de campo distante [8]. 

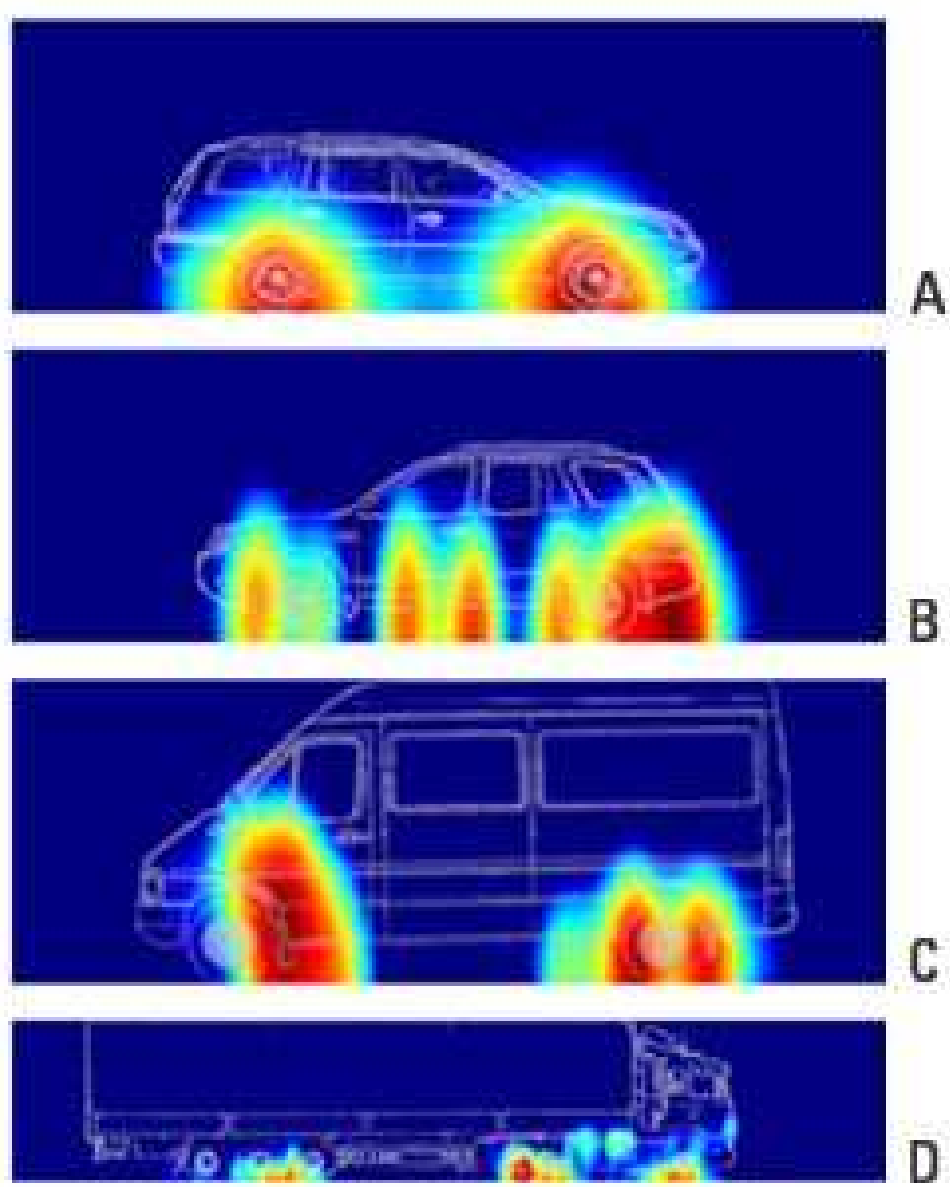

Figura 4 - Exemplo de imagem acústica gerada na análise de ruído de veículos. Imagem modificada de [2].

Existem outras técnicas para estimação de campos acústicos. A mais conhecida é chamada holografia de campo próximo $\left(\mathrm{NAH}^{1}\right)[19,8]$. Este método estima um campo acústico tridimensional no interior de um volume realizando medidas sobre uma superfície que envolva esse mesmo volume e consiste em resolver equações diferenciais parciais de campo próximo usando os dados como condições de contorno. No entanto, este método exige um grande número de amostras, precisão no posicionamento dos sensores, a amostragem deve ser feita o mais próximo possível das fontes e o processo deve ser estacionário durante as medições. Todas essa exigências tornam a aplicação de NAH inconveniente e até impossível para algumas aplicações, além de limitarem a precisão do método [8].

Já no caso das imagens acústicas, o arranjo pode estimar tanto em campo próximo quanto em campo distante. O autor de [8] compara uma imagem acústica a uma fotografia, pois o registro de ondas incidentes em um intervalo de tempo dá origem a uma imagem. Outros trabalhos utilizam arranjos esféricos e decomposição em ondas planas para gerar colormaps [20, 21, 22, 3, 23], isto é, diagramas de intensidade sonora mapeados para cores 


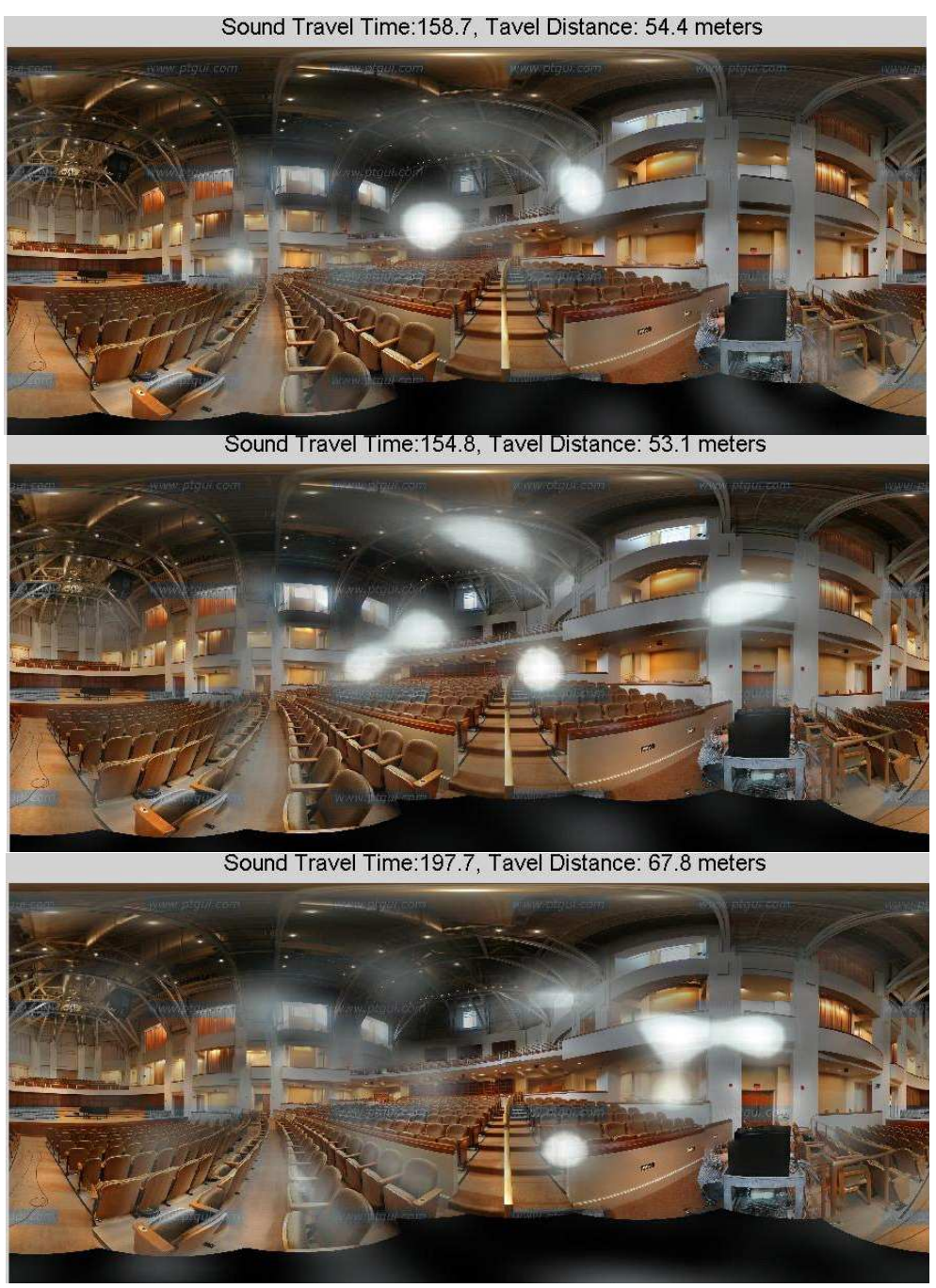

Figura 5 - Exemplo de imagem acústica gerada na análise de reflexão de uma sala de concertos [3].

ou intensidades luminosas em um imagem preto e branca. Esse método permite sobrepor os diagramas de intensidade com imagens do ambiente, possibilitando a verificação visual das direções de propagação do som com maior potência conforme Figura 5, em que os pontos brancos representam as direções de propagação das ondas sonoras chegando a um arranjo de geometria esférica. Estes dados permitem realizar localização dinâmica de fontes, análise de espalhamentos, reflexões, etc [8].

Imagens acústicas são ainda utilizadas na indústria em aplicações como: distribuição de ruído em túneis de vento devido ao fluxo de ar [24, 25]; medição de ruído gerado por turbinas de avião [26]; medição de ruído gerado por turbinas de vento [27]; e medição de ruído e detecção de turbulências de aviões em vôo [28, 29]. 


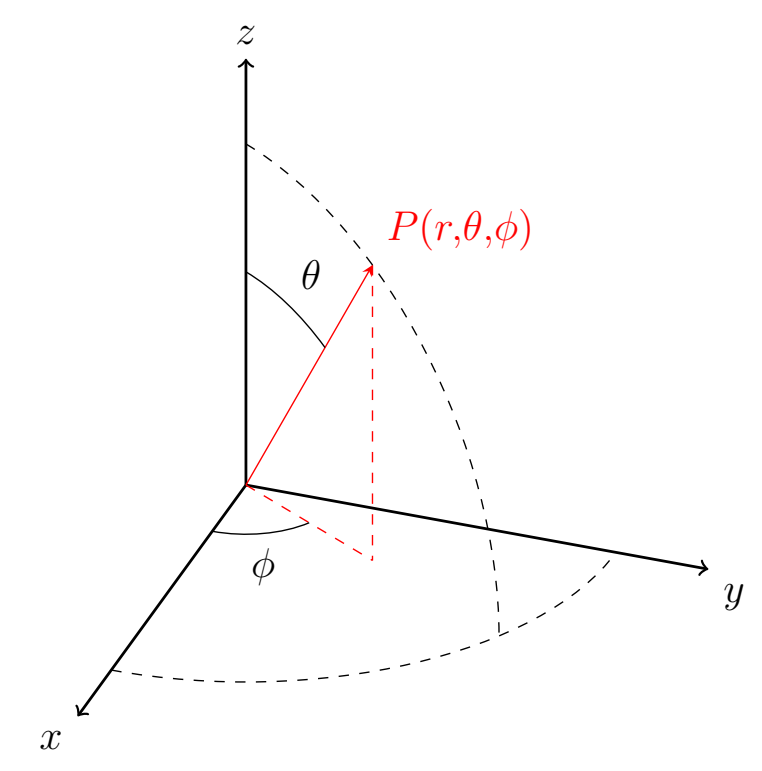

Figura 6 - Sistema de coordenadas esféricas.

\subsection{Modelo acústico}

Para gerar imagens acústicas utilizamos dois parâmetros para obter uma correspondência entre a parametrização de $\mathbf{k}$ e as coordenadas de um pixel da imagem. Lembremos que $\mathbf{k}$ está relacionado com $\mathbf{u}$, conforme definido na seção 2.1, e que os arranjos possuem suas características espaciais incorporadas no vetor diretor $\mathbf{v}_{\mathbf{k}}(\mathbf{k})$.

A direção de visada do arranjo foi definida como

$$
\mathbf{u}=\left[\begin{array}{c}
\sin \theta \cos \phi \\
\sin \theta \sin \phi \\
\cos \theta
\end{array}\right]
$$

em que $\theta$ é o ângulo de elevação e $\phi$ é o ângulo de azimute indicados na Figura 6.

Fazendo

$$
\begin{aligned}
& u_{x}(\theta, \phi)=\sin \theta \cos \phi, \\
& u_{y}(\theta, \phi)=\sin \theta \sin \phi,
\end{aligned}
$$

chegamos a

$$
\mathbf{u}=\left[\begin{array}{c}
u_{x} \\
u_{y} \\
\sqrt{1-u_{x}^{2}-u_{y}^{2}}
\end{array}\right], u_{x}^{2}+u_{y}^{2} \leq 1
$$

Essa parametrização permite representar as direções de chegada com as coordenadas $\left(u_{x}, u_{y}\right) \in U=[-1,1]^{2}, u_{x}^{2}+u_{y}^{2} \leq 1$. Nos referimos ao conjunto $U$ como espaço $U$.

Uma parametrização uniforme - tomada em uma grade retangular por exemplo — no espaço $U$ é desejável, pois torna o padrão de potência ${ }^{2}$ do beamformer, que foi $\overline{2}$ Isto é, sua resposta impulsiva espacial também chamada point spread function (PSF). 


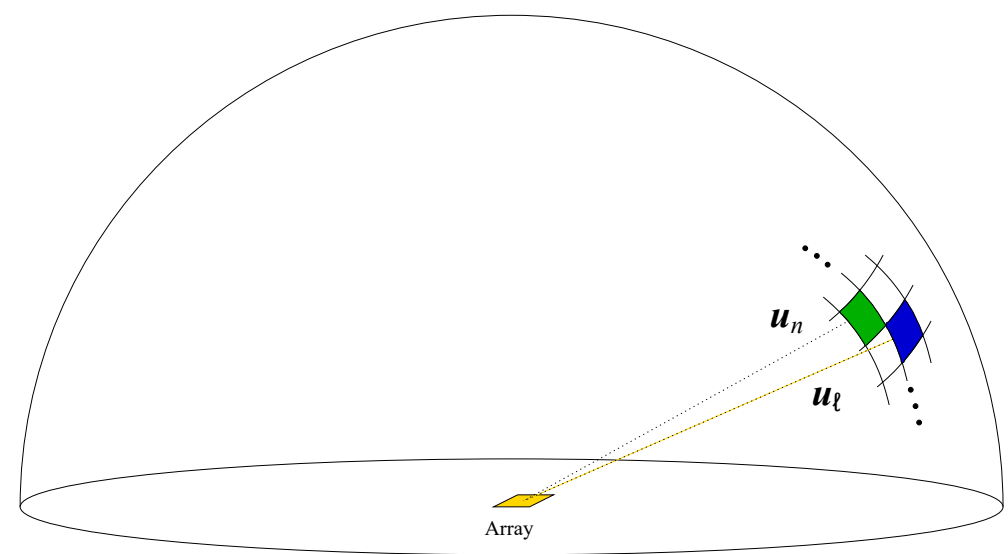

Figura 7 - Amostragem do espaço $U[4]$.

utilizado na formação da imagem, invariante a translações. Isto permite a utilização de transformadas rápidas para o cálculo de imagens acústicas [16]. No entanto, existem ainda otimizações que necessitam apenas de parametrizações cartesianas que não precisam ser uniformes. Portanto, a parametrização escolhida é um importante fator que determina os métodos de aceleração de cálculo de imagens acústicas e deve ser cuidadosamente considerada dependendo da aplicação.

Considerando um arranjo de $N$ sensores acústicos com coordenadas $\mathbf{p}_{0}, \mathbf{p}_{1}, \ldots, \mathbf{p}_{N-1} \in$ $\mathbb{R}^{3}$ e fazendo $\mathbf{k}=-\frac{\omega}{c} \mathbf{u}$ na equação $(2.20)$, chega-se ao vetor diretor do arranjo dado por

$$
\mathbf{v}(\omega, \mathbf{u})=\left[\begin{array}{c}
e^{j \omega \mathbf{u}^{T} \mathbf{p}_{0} / c} \\
e^{j \omega \mathbf{u}^{T} \mathbf{p}_{1} / c} \\
\vdots \\
e^{j \omega \mathbf{u}^{T} \mathbf{p}_{N-1} / c}
\end{array}\right]
$$

Discretizamos o espaço de possíveis direções de visada $\mathbf{u}$ do arranjo e associamos a cada direção escolhida um modelo de fonte pontual (Figura 7). Daí, quanto maior for o número de direções escolhido, ou quando o número for suficientemente grande (a ser determinado pela aplicação), obteremos uma representação fiel do campo acústico incidente com uma margem de erro aceitável (também a ser determinada pela aplicação).

Considere uma discretização com $M$ direções $\mathbf{u}_{0}, \mathbf{u}_{1}, \ldots, \mathbf{u}_{M-1}$. Podemos definir uma matriz cujas colunas correspondem aos vetores diretores para cada direção $\mathbf{u}$ amostrada no espaço $U=[-1,1]^{2}$ com uma parametrização definida por

$$
\mathbf{V}(\omega)=\left[\begin{array}{llll}
\mathbf{v}\left(\omega, \mathbf{u}_{0}\right) & \mathbf{v}\left(\omega, \mathbf{u}_{1}\right) & \cdots & \mathbf{v}\left(\omega, \mathbf{u}_{M-1}\right)
\end{array}\right]
$$

em que $\mathbf{V}(\omega)$ possui dimensões $N \times M$. 
Seja

$$
\mathbf{f}(\omega)=\left[\begin{array}{c}
f_{0}(\omega) \\
f_{1}(\omega) \\
\vdots \\
f_{M-1}(\omega)
\end{array}\right]
$$

o sinal emitido pelas $M$ fontes pontuais no domínio da frequência para uma determinada frequência $\omega$. O sinal $\mathbf{x}(\omega)$ medido nos microfones será a soma dos sinais de todas as fontes atrasados de acordo com as posições dos microfones:

$$
\mathbf{x}(\omega)=\left[\begin{array}{llll}
\mathbf{v}\left(\omega, \mathbf{u}_{0}\right) & \mathbf{v}\left(\omega, \mathbf{u}_{1}\right) & \cdots & \mathbf{v}\left(\omega, \mathbf{u}_{M-1}\right)
\end{array}\right]\left[\begin{array}{c}
f_{0}(\omega) \\
f_{1}(\omega) \\
\vdots \\
f_{M-1}(\omega)
\end{array}\right]=\mathbf{V}(\omega) \mathbf{f}(\omega)
$$

em que $\mathbf{x}(\omega)$ possui dimensões $N \times 1$.

Considere que os sinais nos microfones são amostrados e segmentados em $L$ quadros com $K$ amostras cada e a FFT das amostras é tomada. Assuma que existe um ruído aditivo, então o vetor de saída do arranjo para uma única frequência $\omega_{k}$ é dado por

$$
\mathbf{x}\left(\omega_{k}\right)=\mathbf{V}\left(\omega_{k}\right) \mathbf{f}\left(\omega_{k}\right)+\boldsymbol{\eta}\left(\omega_{k}\right), k=0,1, \ldots, K-1
$$

em que

$$
\boldsymbol{\eta}\left(\omega_{k}\right)=\left[\begin{array}{c}
\eta_{0}\left(\omega_{k}\right) \\
\eta_{1}\left(\omega_{k}\right) \\
\vdots \\
\eta_{N-1}\left(\omega_{k}\right)
\end{array}\right]
$$

é o ruído aditivo.

A matriz espectral do arranjo para cada frequência $k$ é dada por

$$
\mathbf{S}_{\mathbf{x}}\left(\omega_{k}\right)=E\left\{\mathbf{x}\left(\omega_{k}\right) \mathbf{x}^{H}\left(\omega_{k}\right)\right\}
$$

Sejam $\mathbf{x}_{0}\left(\omega_{k}\right), \ldots, \mathbf{x}_{L-1}\left(\omega_{k}\right)$ os sinais medidos nos sensores para os $L$ quadros no domínio da frequência, também chamados de snapshots, então a matriz espectral pode ser estimada usando

$$
\mathbf{S}_{\mathbf{x}}\left(\omega_{k}\right) \cong \frac{1}{L} \sum_{l=0}^{L-1} \mathbf{x}_{l}\left(\omega_{k}\right) \mathbf{x}_{l}^{H}\left(\omega_{k}\right)
$$

Substituir a equação (4.12) na equação (4.14) leva a

$$
\mathbf{S}_{\mathbf{x}}\left(\omega_{k}\right)=\mathbf{V}\left(\omega_{k}\right) E\left\{\mathbf{f}\left(\omega_{k}\right) \mathbf{f}^{H}\left(\omega_{k}\right)\right\} \mathbf{V}^{H}\left(\omega_{k}\right)+\sigma^{2} \mathbf{I},
$$

em que $\sigma^{2}=E\left\{\eta_{i}\left(\omega_{k}\right) \eta_{i}^{*}\left(\omega_{k}\right)\right\}$, assumindo ruído branco e descorrelacionado com as fontes. 
Assuma que as fontes pontuais localizadas nas direções $\mathbf{u}_{m}$ são descorrelacionadas. Ao admitir que as fontes são descorrelacionadas, estamos dizendo que os termos fora da diagonal de $\mathbf{S}_{\mathbf{f}}\left(\omega_{k}\right)=E\left\{\mathbf{f}\left(\omega_{k}\right) \mathbf{f}^{H}\left(\omega_{k}\right)\right\}$ são iguais a zero, ou seja, a matriz $\mathbf{S}_{\mathbf{f}}\left(\omega_{k}\right)$ é diagonal. Perceba que os termos da diagonal são da forma $\left[\mathbf{S}_{\mathbf{f}}\left(\omega_{k}\right)\right]_{m m}=E\left\{\left|f_{m}\left(\omega_{k}\right)\right|^{2}\right\}$, portanto representam a potência emitida por cada uma das fontes e são podem ser mapeados diretamente para a imagem acústica.

Seja $\mathbf{u}_{0}, \mathbf{u}_{1}, \ldots, \mathbf{u}_{M-1}$ uma enumeração de todas as coordenadas de direções no espaço $U$ numa grade $M_{x} \times M_{y}$ (totalizando $M=M_{x} M_{y}$ direções), $\mathbf{v}\left(\mathbf{u}_{m}\right)$ o vetor diretor do arranjo na direção $\mathbf{u}_{m}$ e $y\left(\mathbf{u}_{m}\right)=E\left\{\left|f_{m}\left(\omega_{k}\right)\right|^{2}\right\}$ a potência da fonte com direção $\mathbf{u}_{m}$. Então, a partir de (4.16), podemos escrever

$$
\mathbf{S}=\sum_{m=0}^{M-1} y\left(\mathbf{u}_{m}\right) \mathbf{v}\left(\mathbf{u}_{m}\right) \mathbf{v}^{H}\left(\mathbf{u}_{m}\right),
$$

em que $\mathbf{S}=\mathbf{S}_{\mathbf{x}}$ e omitimos a frequência $\omega_{k}$ para simplificar a notação.

Seja $\mathbf{Y}$ uma imagem digital $M_{x} \times M_{y}$ representando os valores de $y\left(\mathbf{u}_{m}\right)$ (a imagem acústica) organizados a partir de uma parametrização (ordenação) das direções $\mathbf{u}_{m}$. As colunas de $\mathbf{Y}$ correspondem a linhas verticais de pixels amostrados arbitrariamente e suas linhas correspondem a linhas horizontais de pixels amostrados arbitrariamente. Definimos

$$
\mathbf{y}=\operatorname{vec}\{\mathbf{Y}\}=\operatorname{vecd}\left\{\mathbf{S}_{\mathbf{f}}\right\}=\left[\begin{array}{c}
y\left(\mathbf{u}_{0}\right) \\
y\left(\mathbf{u}_{1}\right) \\
\vdots \\
y\left(\mathbf{u}_{M-1}\right)
\end{array}\right]
$$

e

$$
\mathbf{s}=\operatorname{vec}\{\mathbf{S}\}
$$

em que os operadores $\operatorname{vec}\{\cdot\}$ e vecd $\{\cdot\}$ são definidos a seguir.

Definição 1 operador vec cria um vetor coluna a partir de uma matriz $\mathbf{A}$ empilhando os vetores coluna de $\mathbf{A}=\left[\begin{array}{llll}\mathbf{a}_{1} & \mathbf{a}_{2} & \cdots & \mathbf{a}_{n}\end{array}\right]$ um sobre o outro:

$$
\operatorname{vec}\{\mathbf{A}\}=\left[\begin{array}{c}
\mathbf{a}_{1} \\
\mathbf{a}_{2} \\
\vdots \\
\mathbf{a}_{n}
\end{array}\right] \text {. }
$$

Definição 2 O operador vecd cria um vetor coluna a partir de uma matriz diagonal

$$
\mathbf{A}=\left[\begin{array}{llll}
a_{11} & & & \\
& a_{22} & & \\
& & \ddots & \\
& & & a_{n n}
\end{array}\right]
$$


empilhando os elementos da diagonal de $\mathbf{A}$ um sobre o outro:

$$
\operatorname{vecd}\{\mathbf{A}\}=\left[\begin{array}{c}
a_{11} \\
a_{22} \\
\vdots \\
a_{n n}
\end{array}\right]
$$

Vetorizar a equação (4.17) leva a

$$
\begin{aligned}
\mathbf{s}=y\left(\mathbf{u}_{0}\right) \operatorname{vec}\left\{\mathbf{v}\left(\mathbf{u}_{0}\right) \mathbf{v}^{H}\left(\mathbf{u}_{0}\right)\right\}+y\left(\mathbf{u}_{1}\right) \operatorname{vec}\left\{\mathbf{v}\left(\mathbf{u}_{1}\right) \mathbf{v}^{H}\left(\mathbf{u}_{1}\right)\right\} & +\ldots \\
& +y\left(\mathbf{u}_{M-1}\right) \operatorname{vec}\left\{\mathbf{v}\left(\mathbf{u}_{M-1}\right) \mathbf{v}^{H}\left(\mathbf{u}_{M-1}\right)\right\}
\end{aligned}
$$

ou, em forma matricial,

$$
\mathbf{s}=\left[\operatorname{vec}\left\{\mathbf{v}\left(\mathbf{u}_{0}\right) \mathbf{v}^{H}\left(\mathbf{u}_{0}\right)\right\} \quad \cdots \quad \operatorname{vec}\left\{\mathbf{v}\left(\mathbf{u}_{M-1}\right) \mathbf{v}^{H}\left(\mathbf{u}_{M-1}\right)\right] \mathbf{y} .\right.
$$

Podemos, então, escrever a equação (4.17) como uma transformação linear, tal que

$$
\mathrm{s}=\mathrm{Ay}
$$

A equação (4.23) é o ponto de partida para vários métodos de cálculo de imagens acústicas, inclusive métodos eficientes e acelerados por transformadas, e a matriz $\mathbf{A}$ pode ser expressa em termos do vetor diretor da matriz [16].

A partir da equação (4.18) e considerando a equação (4.23), é interessante expressar A em forma fechada a partir do vetor diretor do arranjo $\mathbf{v}\left(\mathbf{u}_{m}\right)$, pois $\mathbf{A}$ será utilizada no cálculo de quase todos os algoritmos abordados nas próximas seções.

Como

$$
\operatorname{vec}\left\{\mathbf{v}\left(\mathbf{u}_{m}\right) \mathbf{v}^{H}\left(\mathbf{u}_{m}\right)\right\}=\left[\begin{array}{c}
\mathbf{v}\left(\mathbf{u}_{m}\right) v_{0}^{*}\left(\mathbf{u}_{m}\right) \\
\mathbf{v}\left(\mathbf{u}_{m}\right) v_{1}^{*}\left(\mathbf{u}_{m}\right) \\
\vdots \\
\mathbf{v}\left(\mathbf{u}_{m}\right) v_{N-1}^{*}\left(\mathbf{u}_{m}\right)
\end{array}\right]
$$

então podemos escrever

$$
\begin{aligned}
\mathbf{s} & =\mathbf{A y} \\
& =\left[\begin{array}{cccc}
\mathbf{v}\left(\mathbf{u}_{0}\right) v_{0}^{*}\left(\mathbf{u}_{0}\right) & \mathbf{v}\left(\mathbf{u}_{1}\right) v_{0}^{*}\left(\mathbf{u}_{1}\right) & \cdots & \mathbf{v}\left(\mathbf{u}_{M-1}\right) v_{0}^{*}\left(\mathbf{u}_{M-1}\right) \\
\mathbf{v}\left(\mathbf{u}_{0}\right) v_{1}^{*}\left(\mathbf{u}_{0}\right) & \mathbf{v}\left(\mathbf{u}_{1}\right) v_{1}^{*}\left(\mathbf{u}_{1}\right) & \cdots & \mathbf{v}\left(\mathbf{u}_{M-1}\right) v_{1}^{*}\left(\mathbf{u}_{M-1}\right) \\
\vdots & \vdots & & \vdots \\
\mathbf{v}\left(\mathbf{u}_{0}\right) v_{N-1}^{*}\left(\mathbf{u}_{0}\right) & \mathbf{v}\left(\mathbf{u}_{1}\right) v_{N-1}^{*}\left(\mathbf{u}_{1}\right) & \cdots & \mathbf{v}\left(\mathbf{u}_{M-1}\right) v_{N-1}^{*}\left(\mathbf{u}_{M-1}\right)
\end{array}\right] \mathbf{y} \\
& =\left[\begin{array}{llll}
\mathbf{v}^{*}\left(\mathbf{u}_{0}\right) \otimes \mathbf{v}\left(\mathbf{u}_{0}\right) & \mathbf{v}^{*}\left(\mathbf{u}_{1}\right) \otimes \mathbf{v}\left(\mathbf{u}_{1}\right) & \cdots & \mathbf{v}^{*}\left(\mathbf{u}_{M-1}\right) \otimes \mathbf{v}\left(\mathbf{u}_{M-1}\right)
\end{array}\right] \mathbf{y},
\end{aligned}
$$

em que $v_{n}(\mathbf{u})$ é o $n$-ésimo elemento do vetor diretor $\mathbf{v}(\mathbf{u})$ e $\otimes$ é o produto de Kronecker, definido a seguir [30]. 
Definição 3 O produto de Kronecker, também denominado produto direto e produto tensorial de matrizes, de $\mathbf{A}=\left[a_{i j}\right] \in M_{m, n}(\mathbb{F})$ e $\mathbf{B}=\left[b_{i j}\right] \in M_{p, q}(\mathbb{F})$ denotado por $\mathbf{A} \otimes \mathbf{B}$ é definido como a matriz de bloco

$$
\mathbf{A} \otimes \mathbf{B}=\left[\begin{array}{ccc}
a_{11} \mathbf{B} & \cdots & a_{1 n} \mathbf{B} \\
\vdots & \ddots & \vdots \\
a_{m 1} \mathbf{B} & \cdots & a_{m n} \mathbf{B}
\end{array}\right] \in M_{m p, n q}(\mathbb{F})
$$

em que $M_{m, n}(\mathbb{F})$ é o espaço de matrizes $m \times n$ definido sobre o corpo $\mathbb{F}$. Em geral, $\mathbb{F}=\mathbb{C}$ ou $\mathbb{R}$.

Cada coluna da matriz A, de acordo com a equação (4.25), consiste no produto de Kronecker das colunas correspondentes das matrizes $\mathbf{V}^{*}$ e $\mathbf{V}$. Essa estrutura corresponde a um produto matricial específico chamado produto de Khatri-Rao [31, 6], definido a seguir.

Definição 4 Considere as matrizes $\mathbf{A}$ de ordem $p \times r$ e $\mathbf{B}$ de ordem $q \times r$; sejam $\mathbf{a}_{1}, \ldots, \mathbf{a}_{r}$ $e \mathbf{b}_{1}, \ldots, \mathbf{b}_{r}$ vetores que correspondem às colunas de $\mathbf{A} e \mathbf{B}$, respectivamente. O produto de Khatri-Rao é definido como a matriz de bloco

$$
\mathbf{A} \odot \mathbf{B}=\left[\begin{array}{llll}
\mathbf{a}_{1} \otimes \mathbf{b}_{1} & \mathbf{a}_{2} \otimes \mathbf{b}_{2} & \cdots & \mathbf{a}_{r} \otimes \mathbf{b}_{r}
\end{array}\right]
$$

que é uma matriz de ordem $p q \times r$.

Portanto, podemos escrever

$$
\mathbf{A}=\mathbf{V}^{*} \odot \mathbf{V}
$$

e

$$
\mathbf{s}=\left(\mathbf{V}^{*} \odot \mathbf{V}\right) \mathbf{y}
$$

\subsection{Métodos para imageamento acústico}

Baseado em $[4,8]$, nesta seção serão apresentados os métodos mais comuns para imageamento acústico: filtragem espacial e métodos de deconvolução, nos quais está incluído o estado da arte.

\subsubsection{Filtragem espacial}

Um filtro espacial é implementado como a soma ponderada dos sinais capturados pelos microfones do arranjo tal que a saída do sistema é

$$
Z=\mathbf{w}^{H} \mathbf{x}
$$


em que $\mathbf{w}=\left[\begin{array}{llll}w_{0} & w_{1} & \cdots & w_{N-1}\end{array}\right]^{T}$ é um vetor de pesos complexos. Veja que a equação anterior corresponde a uma soma ponderada escrita em forma vetorial com $\mathbf{w}^{H}$ sendo um vetor linha e $\mathbf{x}$ um vetor coluna, ambos com o mesmo número de elementos, portanto, seu produto é um escalar escrito na forma de somas de produtos de um elemento de $\mathbf{w}^{H}$ e um elemento de $\mathbf{x}$.

\subsubsection{Beamformer determinístico}

Existem diversas maneiras de se calcular w, sendo a mais simples delas o uso de beamformers determinísticos. No chamado beamformer convencional ou de Bartlett, w é escolhido de forma a maximizar a saída do filtro espacial quando uma onda plana incide no arranjo vinda da direção $\mathbf{u}$.

Assumindo o sinal vindo da direção $\mathbf{u}_{m}$ corrompido por ruído espacial branco teremos

$$
\mathbf{x}(t)=\mathbf{v}\left(\mathbf{u}_{m}\right) f(t)+\boldsymbol{\eta}(t)
$$

De acordo com [32], o problema de maximizar a potência de saída pode ser formulado como

$$
\max _{\mathbf{w}} E\left\{\mathbf{w}^{H} \mathbf{x}(t) \mathbf{x}^{H}(t) \mathbf{w}\right\}=\max _{\mathbf{w}}\left\{E|f(t)|^{2}\left|\mathbf{w}^{H} \mathbf{v}(\mathbf{u})\right|^{2}+\sigma_{n}^{2}\|\mathbf{w}\|^{2}\right\}
$$

Para evitar a solução trivial $\|\mathbf{w}\| \rightarrow \infty$, adiciona-se a restrição $\|\mathbf{w}\|=1$, e, através do uso da desigualdade de Cauchy-Schwarz, chega-se ao resultado

$$
\mathbf{w}_{B F}(\mathbf{u})=\frac{\mathbf{v}(\mathbf{u})}{\|\mathbf{v}(\mathbf{u})\|}=\frac{\mathbf{v}(\mathbf{u})}{\sqrt{N}} .
$$

Em resumo, o beamformer de Bartlett aplica um atraso aos sinais capturados pelos sensores do arranjo de forma que os sinais chegando da direção u são adicionados construtivamente. Como este método não depende das estatísticas do sinal incidente, mas somente da geometria do arranjo e da direção de chegada, então é determinístico.

O beamformer DAS discutido na seção 2.1 é equivalente ao beamformer de Bartlett a não ser por um ganho escalar. Ele busca compensar o atraso relativo entre cada sensor e então calcula a média dos sinais resultantes:

$$
\mathbf{w}_{D A S}(\mathbf{u})=\frac{1}{N} \mathbf{v}(\mathbf{u})=\frac{\mathbf{v}(\mathbf{u})}{\mathbf{v}^{H}(\mathbf{u}) \mathbf{v}(\mathbf{u})}
$$

Para obter a imagem acústica com a aplicação desses métodos é necessário estimar a intensidade sonora vindo de cada direção u em uma amostragem prévia do espaço, isto é, uma grade pré-definida, para obter um vetor de estimativa da imagem acústica $\hat{\mathbf{y}}$, ou equivalentemente $\hat{\mathbf{Y}}$. Usando um beamformer fixo para cada direção amostrada e assumindo uma estimativa perfeita do sinal, temos

$$
\hat{y}\left(\mathbf{u}_{m}\right)=E\left\{\left|\mathbf{w}^{H}\left(\mathbf{u}_{m}\right) \mathbf{x}\right|^{2}\right\}=\mathbf{w}^{H}\left(\mathbf{u}_{m}\right) E\left\{\mathbf{x x}^{H}\right\} \mathbf{w}\left(\mathbf{u}_{m}\right)
$$


O valor esperado é aproximado estimando-se $\mathbf{x}$ para um número de $L$ quadros com $K$ amostras cada e tomando a média de $\left|\mathbf{w}^{H}\left(\mathbf{u}_{m}\right) \mathbf{x}\right|^{2}$ considerando os $L$ quadros.

Depois de estabelecidas algumas identidades matriciais, mostraremos na seção 5.3.1 que a equação (4.33), no caso de DAS, equivale a

$$
\hat{\mathbf{y}}=\frac{1}{N^{2}} \mathbf{A}^{H} \mathbf{s}
$$

e que Bartlett é equivalente a

$$
\hat{\mathbf{y}}=\frac{1}{N} \mathbf{A}^{H} \mathbf{s}
$$

Nos referimos a essa formulação como CSM-DAS ${ }^{3}$.

Portanto, a implementação de beamforming convencional, seja ele DAS ou Bartlett, corresponde à implementação direta das duas equações anteriores. Num ambiente de simulação seria necessário estimar o vetor s, calcular a matriz A que depende apenas da geometria do arranjo e proceder com o cálculo direto a partir das equações. Porém, essa formulação vetorizada é conveniente para o uso de transformadas rápidas no cálculo da imagem acústica. Tradicionalmente, a equação (4.33) seria utilizada, como descrito anteriormente.

Também é possível estimar os sinais das fontes $\mathbf{f}$ diretamente fazendo apenas a aplicação do beamformer:

$$
\hat{f}_{m}=\frac{1}{N} \mathbf{v}^{H}\left(\mathbf{u}_{m}\right) \mathbf{x}
$$

ou, em forma matricial,

$$
\hat{\mathbf{f}}=\frac{1}{N} \mathbf{V}^{H} \mathbf{x}
$$

Nos referimos a essa última formulação como X-DAS.

\subsubsection{Beamformers ótimos}

Os beamformers convencionais possuem a vantagem de serem extremamente simples de implementar, requerendo operações e equações simples. Porém, a grande desvantagem destes métodos é a baixa resolução da imagem obtida, isto é, se duas fontes estão posicionadas muito próximas uma da outra, o método não será capaz de discriminá-las. Uma das soluções possíveis para este problema é o uso de beamformers que são estatisticamente ótimos. Usando as estatísticas do campo sonoro incidente no arranjo, representadas através da matriz de autocorrelação dos sinais, estes métodos são capazes de reduzir a influência de fontes próximas entre si resultando em uma imagem de melhor resolução [4].

Em [1] é mostrado que para uma grande classe de beamformers ótimos - incluindo os beamformers minimum variance distortionless response (MVDR), minimum power distortionless response (MPDR), minimum mean square error (MMSE) e maximum signal

3 CSM, do inglês cross spectral matrix. 
to noise (SNR) - o filtro espacial resultante é um beamformer MVDR seguido de um filtro escalar que depende do critério de otimização utilizado.

O beamformer MVDR minimiza a variância do sinal $Z(\omega)$ recebido pelo arranjo e ao mesmo tempo mantém um ganho unitário na direção de interesse, isto é, $\mathbf{w}^{H} \mathbf{v}(\mathbf{u})=1$. Este é um problema de otimização que pode ser resolvido com multiplicadores de Lagrange, como feito em [1], e resulta em

$$
\mathbf{w}_{M V D R}^{H}(\mathbf{u})=\frac{\mathbf{v}^{H}(\mathbf{u}) \mathbf{S}_{n}^{-1}}{\mathbf{v}^{H}(\mathbf{u}) \mathbf{S}_{n}^{-1} \mathbf{v}(\mathbf{u})},
$$

em que $\mathbf{S}_{n}$ é a matriz de autocorrelação do ruído.

A obtenção de $\mathbf{S}_{n}$ para uma imagem acústica em uma aplicação prática necessita de uma medição separada [33]. Se esta medição não for possível de ser realizada, pode-se utilizar o beamformer MPDR substituindo $\mathbf{S}_{n}$ por $\mathbf{S}+\lambda \mathbf{I}$, em que $\lambda \geq 0$ é um parâmetro de regularização escolhido apropriadamente para melhorar o condicionamento da matriz $\mathbf{S}$, tal que

$$
\mathbf{w}_{M P D R}^{H}(\mathbf{u})=\frac{\mathbf{v}^{H}(\mathbf{u})[\mathbf{S}+\lambda \mathbf{I}]^{-1}}{\mathbf{v}^{H}(\mathbf{u})[\mathbf{S}+\lambda \mathbf{I}]^{-1} \mathbf{v}(\mathbf{u})},
$$

em que $\mathbf{S}=E\left\{\mathbf{x x}^{H}\right\}$ é a matriz espectral do sinal recebido pelo arranjo, incluindo o ruído.

A imagem acústica pode ser aproximada pela potência na saída do beamformer MVDR. Com $\mathbf{w}=\mathbf{w}_{M V D R}$ temos

$$
\begin{aligned}
y\left(\mathbf{u}_{m}\right) & =E\left\{\left|\mathbf{w}^{H}\left(\mathbf{u}_{m}\right) \mathbf{x}\right|^{2}\right\}=\mathbf{w}^{H}\left(\mathbf{u}_{m}\right) E\left\{\mathbf{x} \mathbf{x}^{H}\right\} \mathbf{w}\left(\mathbf{u}_{m}\right) \\
& =\frac{\mathbf{v}^{H}\left(\mathbf{u}_{m}\right) \mathbf{S}_{n}^{-1} \mathbf{S} \mathbf{S}_{n}^{-1} \mathbf{v}^{H}\left(\mathbf{u}_{m}\right)}{\left[\mathbf{v}^{H}\left(\mathbf{u}_{m}\right) \mathbf{S}_{n}^{-1} \mathbf{v}\left(\mathbf{u}_{m}\right)\right]^{2}}
\end{aligned}
$$

O caso para MPDR é análogo, bastando fazer $\mathbf{w}=\mathbf{w}_{M P D R}$. Veja que a imagem acústica é gerada da mesma maneira que no caso convencional (Equação (4.33)).

\subsubsection{Métodos de deconvolução}

Como provado por [8], sob hipóteses de fontes descorrelacionadas em campo distante e amostragem uniforme no espaço $U$, a reconstrução de imagens acústicas por beamforming é uma operação aproximadamente linear e invariante por deslocamentos.

Todo sistema linear e invariante por deslocamentos ${ }^{4}$ pode ser representado pela convolução de um sinal de entrada com a resposta a impulso do sistema [34, 10]. Portanto, a imagem acústica reconstruída com uso de técnicas de beamforming consiste na distribuição

$\overline{4}$ De forma rigorosa todo sistema $\sigma$-linear e invariante por deslocamentos pode ser representado por uma convolução de sua entrada com a resposta ao impulso do sistema. Mas nem todo sistema linear invariante por deslocamentos possui essa propriedade. Para definição de $\sigma$-linearidade e provas matematicamente rigorosas veja [34]. 
de fontes sonoras no espaço convoluída com a resposta impulsiva espacial do beamformer, também chamada point spread function ou PSF.

Considerando uma única onda viajando na direção $-\mathbf{u}_{m}$, que o pixel correspondente à direção $\mathbf{u}_{m^{\prime}}$ é estimado pela equação (4.33), e o modelo da equação (4.17) chega-se a

$$
\hat{y}\left(\mathbf{u}_{m^{\prime}}\right)=y\left(\mathbf{u}_{m}\right) \mathbf{w}^{H}\left(\mathbf{u}_{m^{\prime}}\right) \mathbf{v}\left(\mathbf{u}_{m}\right) \mathbf{v}^{H}\left(\mathbf{u}_{m}\right) \mathbf{w}\left(\mathbf{u}_{m^{\prime}}\right) \triangleq y\left(\mathbf{u}_{m}\right) P\left(\mathbf{u}_{m^{\prime}}, \mathbf{u}_{m}\right)
$$

em que $y\left(\mathbf{u}_{m}\right)$ é a potência da fonte que se propaga na direção $-\mathbf{u}_{m}$.

O termo $P\left(\mathbf{u}_{m^{\prime}}, \mathbf{u}_{m}\right)$, que é função apenas de $\mathbf{u}_{m}$ com $\mathbf{u}_{m^{\prime}}$ fixado, é a resposta espacial do arranjo ou sua PSF e descreve o ganho aplicado pelo arranjo a uma onda que se propaga na direção $-\mathbf{u}_{m}$. Apesar de um modelo discreto ter sido considerado, $P\left(\mathbf{u}_{m^{\prime}}, \mathbf{u}_{m}\right)$ é definido para todo o espaço e pode ser interpretado como uma função de amostragem espacial que idealmente deveria ser dada por

$$
P\left(\mathbf{u}_{m^{\prime}}, \mathbf{u}_{m}\right)=\delta_{\mathbf{u}_{m^{\prime}}, \mathbf{u}_{m}}
$$

em que $\delta_{\mathbf{u}_{m^{\prime}}, \mathbf{u}_{m}}$ é tal que $\delta_{\mathbf{u}_{m^{\prime}}, \mathbf{u}_{m}}=1, \mathbf{u}_{m}=\mathbf{u}_{m^{\prime}}$ e $\delta_{\mathbf{u}_{m^{\prime}}, \mathbf{u}_{m}}=0, \mathbf{u}_{m} \neq \mathbf{u}_{m^{\prime}}$. Isto é válido para uma amostragem discreta no espaço $U$; no caso contínuo, trabalharíamos com impulsos de Dirac fazendo $P\left(\mathbf{u}_{m^{\prime}}, \mathbf{u}_{m}\right)=\delta\left(\mathbf{u}_{m^{\prime}}-\mathbf{u}_{m}\right)$. No entanto, como os arranjos de microfones construídos com a tecnologia atual possuem apenas um número limitado de sensores, no máximo algumas centenas, suas PSFs apresentam uma grande largura de feixe e, consequentemente, geram uma imagem desfocada $[4,8]$.

Além disso, PSFs típicas têm aspecto similar a um filtro passa-baixas, sendo esta uma característica altamente indesejável, pois reduz a resolução das imagens e impede a determinação precisa de níveis de potência na presença de fontes distribuídas ou muito próximas umas das outras [8].

Para reduzir o desfocamento apresentado no uso de beamforming, vários métodos de deconvolução foram propostos [35, 36, 37, 38]. Todos usam como entradas a PSF do arranjo e a imagem obtida com uso de beamforming DAS e, em geral, produzem uma aproximação melhor do que aquela obtida apenas usando beamforming [4].

\subsubsection{DAMAS e DAMAS2}

DAMAS ou deconvolution approach for the mapping of acoustic sources é um método muito empregado para deconvolução de imagens acústicas [39]. Assumindo fontes descorrelacionadas, é possível estabelecer uma relação linear entre a imagem obtida com um beamformer DAS e a imagem limpa formada apenas pelas fontes [8].

A partir da equação (4.17), sua forma vetorizada dada pela equação (4.23) tal que 
$y_{m}=y\left(\mathbf{u}_{m}\right)$ e seja $\breve{\mathbf{Y}}$ a imagem estimada com DAS com $\breve{\mathbf{y}}=\operatorname{vec}(\breve{\mathbf{Y}})$, então

$$
\begin{aligned}
\breve{y}_{m^{\prime}} & =\mathbf{v}^{H}\left(\mathbf{u}_{m^{\prime}}\right) \mathbf{S} \mathbf{v}\left(\mathbf{u}_{m^{\prime}}\right) \\
& =\mathbf{v}^{H}\left(\mathbf{u}_{m^{\prime}}\right)\left[\sum_{m=0}^{M-1} y_{m} \mathbf{v}\left(\mathbf{u}_{m}\right) \mathbf{v}^{H}\left(\mathbf{u}_{m}\right)\right] \mathbf{v}\left(\mathbf{u}_{m^{\prime}}\right)
\end{aligned}
$$

tal que

com

$$
\left[\begin{array}{c}
\breve{y}_{0} \\
\breve{y}_{1} \\
\vdots \\
\breve{y}_{M-1}
\end{array}\right]=\left[\begin{array}{cccc}
B_{0,0} & B_{0,1} & \cdots & B_{0, M-1} \\
B_{1,0} & B_{1,1} & \cdots & B_{1, M-1} \\
\vdots & \vdots & & \vdots \\
B_{M-1,0} & B_{M-1,1} & \cdots & B_{M-1, M-1}
\end{array}\right]\left[\begin{array}{c}
y_{0} \\
y_{1} \\
\vdots \\
y_{M-1}
\end{array}\right]
$$

$$
B_{m^{\prime}, m}=\left|\mathbf{v}^{H}\left(\mathbf{u}_{m^{\prime}}\right) \mathbf{v}\left(\mathbf{u}_{m}\right)\right|^{2} .
$$

O algoritmo DAMAS resolve iterativamente a equação (4.44) usando o método de Gauss-Seidel com $\mathbf{y}^{(0)}=0$. Se $\mathbf{B}$ tiver diagonal dominante ou for positiva definida, então $\mathbf{y}^{(k+1)} \rightarrow \mathbf{y}$, isto é, as condições de convergência do método de Gauss-Seidel são satisfeitas e o algoritmo converge. No entanto, B sempre é singular e, por isso, é necessário forçar $\mathbf{y}^{(k+1)}$ a ser não negativo a cada iteração, pois a equação (4.44) não tem solução única caso não haja regularização.

DAMAS produz um resultado significativamente melhor que beamforming convencional, porém demanda muitos recursos computacionais e a convergência geralmente é lenta $[8]$.

Uma melhoria do método foi proposta em [36] e foi denominada DAMAS2. O novo método assume que a imagem obtida com DAS seja a convolução da imagem limpa com a PSF do beamformer.

Se levarmos em conta o fato de que $P$ é invariante a deslocamentos para o caso de distribuição uniforme no espaço $U[8,36,40]$, vale que

$$
P\left(\mathbf{u}_{m^{\prime}}, \mathbf{u}_{m}\right)=P\left(\mathbf{u}_{m^{\prime}}+\Delta, \mathbf{u}_{m}+\Delta\right)
$$

e podemos escrever

$$
\hat{\mathbf{Y}}=\mathbf{Y} * \mathbf{P}
$$

em que * denota a convolução bidimensional e $\mathbf{P}=\mathbf{P}\left(\mathbf{u}_{m}\right)$ é uma representação matricial da PSF que obedece o mesmo mapeamento de direções que a matriz $\mathbf{Y}$ para todas as direções $\mathbf{u}_{m^{\prime}}$. Tomamos $\mathbf{P}$ como a PSF correspondente a uma fonte se propagando na direção central $\mathbf{u}_{c}=\left[\begin{array}{lll}0 & 0 & 1\end{array}\right]^{T}$ e deslocada de acordo com a equação (4.46). Isso é uma aproximação, já que faltam pixels da PSF quando fazemos o deslocamento para o cálculo da convolução bidimensional na versão discretizada da PSF. Nos casos em que a direção $\mathbf{u}_{c}$ não esteja presente na amostragem específica do espaço $U$, basta utilizar a direção mais próxima do centro da imagem [40]. 
Sejam $\breve{\mathbf{Y}}$ a imagem obtida com DAS, $\mathbf{P}$ uma versão discretizada da PSF do beamformer em forma de matriz, $\mathbf{Y}$ a imagem limpa e $\breve{\mathbf{Y}}=\mathbf{P} * \mathbf{Y}$, DAMAS2 aproxima $\mathbf{Y}$ iterando

$$
\mathbf{Y}^{(k+1)}=\max \left\{\mathbf{Y}^{(k)}+\frac{1}{a}\left[\breve{Y}-\left(\mathbf{P} * \mathbf{Y}^{(k)}\right)\right], 0\right\}
$$

em que $\max \{\cdot, \cdot\}$ retorna o máximo ponto a ponto, $a=\sum_{i, j}[\mathbf{P}]_{i, j}, \mathbf{Y}^{(0)}=\mathbf{0}$ e a convolução usa zero padding.

DAMAS2 é muito mais eficiente que DAMAS pois atualiza todas as variáveis ao mesmo tempo e exige apenas uma convolução, que pode ser acelerada por FFT, para atualizar o sistema todo. Além disso, DAMAS2 é capaz de produzir uma imagem deconvoluída e precisa mesmo no caso de fontes distribuídas, algo que não era possível com beamforming [4]. DAMAS2 é considerado o estado da arte para estimar imagens acústicas de forma eficiente computacionalmente.

DAMAS2 também pode ser implementado a partir da matriz A discutida anteriormente. Para isso, observa-se que, para o beamformer DAS,

$$
P\left(\mathbf{u}_{m^{\prime}}, \mathbf{u}_{m}\right)=\frac{1}{N}\left|\mathbf{v}^{H}\left(\mathbf{u}_{m^{\prime}}\right) \mathbf{v}\left(\mathbf{u}_{m}\right)\right|^{2} .
$$

Pode-se montar a matriz $\left[\mathbf{P}\left(\mathbf{u}_{m}\right)\right]_{i j}=P\left(\mathbf{u}_{i j}, \mathbf{u}_{m}\right)$, em que $\mathbf{u}_{m}$ é a direção de propagação da onda incidente e $\mathbf{u}_{m^{\prime}}$ são as direções $\left\{\mathbf{u}_{0}, \mathbf{u}_{1}, \ldots, \mathbf{u}_{M-1}\right\}$ mapeadas por $m^{\prime}=m^{\prime}(i, j)$ em ordem correspondente aos índices de $\mathbf{Y}$, de forma que

$$
\mathbf{A}^{H} \mathbf{A}=\left[\operatorname{vec}\left\{\mathbf{P}\left(\mathbf{u}_{0}\right)\right\} \quad \operatorname{vec}\left\{\mathbf{P}\left(\mathbf{u}_{1}\right)\right\} \quad \cdots \quad \operatorname{vec}\left\{\mathbf{P}\left(\mathbf{u}_{M-1}\right)\right\}\right] .
$$

Agora, o cálculo da convolução $\mathbf{B}=\mathbf{P} * \mathbf{Y}^{(k)}$, depois de se fazer $\mathbf{b}=\operatorname{vec}(\mathbf{B})$, se torna

$$
\mathbf{b}=\mathbf{A}^{H} \mathbf{A} \operatorname{vec}(\mathbf{Y})=\mathbf{A}^{H} \mathbf{A y} .
$$

Resta decidir o valor adequado do parâmetro $a$, que tem influência direta na velocidade de convergência do algoritmo. Como a matriz $\mathbf{A}^{H} \mathbf{A}$ possui $M$ PSFs - uma para cada coluna - faz sentido redefinir a como

$$
a=\frac{1}{M} \sum_{i, j=0}^{M-1}\left[\mathbf{A}^{H} \mathbf{A}\right]_{i j},
$$

isto é, a média da soma de todas as PSFs.

\subsubsection{CLEAN}

Este algoritmo foi inicialmente proposto para reconstrução de imagens astronômicas por [41] e depois estendido para banda larga usando o modelo de snapshots por [38].

CLEAN recebe a imagem suja $\breve{\mathbf{Y}}$, a PSF $\mathbf{P}$ do arranjo e um escalar $0<\gamma \ll 1$ e retorna uma estimativa da imagem limpa Y. O algoritmo estima iterativamente as 
coordenadas da fonte sonora dominante utilizando o máximo de $\mathbf{G}=\mathbf{P} * \breve{\mathbf{Y}}$. A cada iteração, uma fração $\gamma$ do valor máximo de $\mathbf{G}_{n_{\max }, m_{\max }}$, com $\left(n_{\max }, m_{\max }\right)$ correspondendo ao índice do elemento máximo, é adicionada a $\mathbf{Y}_{n_{\max }, m_{\max }}$. Depois, a imagem suja é atualizada subtraindo $\gamma \mathbf{G}_{n_{\max }, m_{\max }}$ de $\breve{\mathbf{Y}}_{n_{\max }, m_{\max }}$.

Este método funciona bem apenas para fontes pontuais, no entanto, imagens acústicas em aplicações práticas são caracterizadas por fontes distribuídas. Além disso, CLEAN tende a causar muitos artefatos na imagem, funcionando bem apenas quando as fontes se encontram na região central da imagem [8]. 


\section{Métodos acelerados para imageamento acústico}

O problema principal, relativo ao custo computacional, dos métodos para cálculo de imagens acústicas é que eles requerem repetidos cálculos de produtos de matrizes da forma Ay e $\mathbf{V x}$. Como a matriz A é grande para aplicações em tempo real, esses métodos se tornam muito pesados computacionalmente. Por exemplo, usando 64 sensores e estimando uma imagem com $256 \times 256$ pixels, a matriz A seria $64^{2} \times 256^{2}=4096 \times 65536$. Uma alternativa é utilizar as propriedades de $\mathbf{A}$ para calcular os produtos de forma mais eficiente. Já a matriz $\mathbf{V}$, mesmo sendo consideravelmente menor que a matriz $\mathbf{A}$, também possui propriedades que permitem uma redução significativa na quantidade de operações aritméticas.

Serão apresentados três métodos para aceleração dos cálculos de imagens acústicas: a NNFFT ou nonequispaced in time and frequency fast Fourier transform, que pode ser aplicada a qualquer geometria de arranjo ou esquema de amostragem; a NFFT ou nonequispaced fast Fourier transform, válida para qualquer arranjo plano e amostragem uniforme das direções de visada; e a transformada de arranjo de Kronecker ou KAT ${ }^{1}$, válida para arranjos planos e de geometria separável e amostragem separável de direções de visada.

\subsection{NFFT e NNFFT}

Neste trabalho utilizaremos a biblioteca NFFT3 [42] para o cálculo das transformadas NFFT e NNFFT. A NFFT é um algoritmo rápido aproximado para o cálculo da NDFT (nonequispaced discrete Fourier transform). Antes de aplicar o algoritmo ao cálculo de imagens acústicas, precisamos definir as transformadas. Seguiremos a notação de [42, 8].

Seja o toro de dimensão $d$

$$
\mathbb{T}^{d} \triangleq\left\{\mathbf{x} \in \mathbb{R}^{d}:-\frac{1}{2} \leq x_{l}<\frac{1}{2}, l=0,1, \ldots, d\right\}=\left[-\frac{1}{2}, \frac{1}{2}\right)^{d}
$$

O conjunto de pontos espaciais $\mathcal{X} \triangleq\left\{\mathbf{x}_{i} \in \mathbb{T}^{d}: i=0,1, . . N-1\right\}$ obtidos a partir do toro $\mathbb{T}^{d}$ tal que $|\mathcal{X}|=N$ serão os nós nos quais a transformada será amostrada. As frequências $\mathbf{k} \in \mathbb{Z}^{d}$ são representadas pelo conjunto de índices

$$
I_{\mathbf{M}} \triangleq\left\{\mathbf{k} \in \mathbb{Z}^{d}:-\frac{M_{t}}{2} \leq k_{t}<\frac{M_{t}}{2}, t=0,1, \ldots, d-1\right\}=\mathbb{Z}^{d} \cap \prod_{t=0}^{d-1}\left[-\frac{M_{t}}{2}, \frac{M_{t}}{2}\right),
$$

\footnotetext{
1 Do inglês Kronecker array transform.
} 
porém a biblioteca NFFT3 requer a especificação de um vetor de largura de banda $\mathbf{M}$ tal que $M_{t} \in 2 \mathbb{N}, t=0, . ., d$ para o cálculo de $I_{\mathbf{M}}$, que é realizado automaticamente. Essa restrição nos elementos de $\mathbf{M}$ implicará que devemos escolher os números de direções $M_{x}$ e $M_{y}$ pares para o cálculo de imagens acústicas utilizando a NFFT. Note que $\left|I_{\mathbf{M}}\right|=\prod_{t=0}^{d-1} M_{t}=M$.

Sejam $\hat{h}_{\mathbf{k}} \in \mathbb{C}$ os coeficientes de Fourier dados como entrada para o algoritmo. A NDFT é definida como o cálculo do polinômio trigonométrico $h: \mathbb{T}^{d} \rightarrow \mathbb{C}$ tal que $h \in T_{\mathbf{M}} \triangleq \operatorname{span}\left\{e^{-j 2 \pi \mathbf{k}}: \mathbf{k} \in I_{\mathbf{M}}\right\}$ no conjunto de nós $\mathcal{X}$. No nó $\mathbf{x}_{i}$, temos

$$
h_{i}=h\left(\mathbf{x}_{i}\right) \triangleq \sum_{\mathbf{k} \in I_{\mathbf{M}}} \hat{h}_{\mathbf{k}} e^{-j 2 \pi \mathbf{k}^{T} \mathbf{x}_{i}}, i=0,1, \ldots, N-1
$$

Observando as equações para todos os $\mathbf{x}_{i}$, podemos escrever o cálculo em forma matricial:

$$
\mathbf{h}=\mathbf{A} \hat{\mathbf{h}}
$$

em que

$$
\mathbf{h}=\left[\begin{array}{c}
h_{0} \\
h_{1} \\
\vdots \\
h_{N-1}
\end{array}\right], \quad \hat{\mathbf{h}}=\left[\begin{array}{c}
\hat{h}_{0} \\
\hat{h}_{1} \\
\vdots \\
\hat{h}_{M-1}
\end{array}\right], \quad[\mathbf{A}]_{i,:}=\left[\begin{array}{c}
e^{-j 2 \pi \mathbf{k}^{T} \mathbf{x}_{0}} \\
e^{-j 2 \pi \mathbf{k}^{T} \mathbf{x}_{1}} \\
\vdots \\
e^{-j 2 \pi \mathbf{k}^{T} \mathbf{x}_{N-1}}
\end{array}\right]
$$

Note que A é de dimensão $N \times M$.

Muitos dos algoritmos de imagens acústicas vão requerer o cálculo da NFFT adjunta dada por

$$
\hat{\mathrm{g}}=\mathbf{A}^{H} \mathbf{h}, \quad \hat{g}_{\mathbf{k}}=\sum_{i=0}^{N-1} h_{i} e^{j 2 \pi \mathbf{k}^{T} \mathbf{x}_{i}}
$$

Note que a matriz A geralmente não é quadrada, mas mesmo nos casos em que ela é quadrada ela não necessariamente possui inversa, portanto o problema de inversão da NFFT não é trivial e por isso lidamos com a transformada adjunta [42]. A biblioteca NFFT3 inclusive inclui a implementação da transformada adjunta de forma que não precisamos derivar as equações para o caso adjunto: basta apenas utilizar $\mathbf{h}$ como entrada ao invés de $\hat{\mathbf{h}}$.

O cálculo direto da NDFT, por produto matricial, requer $\mathcal{O}(M N)$ MACs complexas. O algoritmo de NFTT implementado na biblioteca NFFT3 consegue reduzir a complexidade para $\mathcal{O}\left(M \log M+|\log \epsilon|^{d} N\right)$, em que $\epsilon$ é a precisão dos cálculos e a constante que multiplica a complexidade depende da quantidade disponível de memória para os cálculos realizados na inicialização do algoritmo. Além disso, a NFFT introduz erros no cálculo da NDFT para reduzir o custo computacional portanto não é um algoritmo exato [42].

Se tivermos $N=M$ nós espaçados uniformemente, o algoritmo se reduz a uma DFT de dimensão $d$. Esse caso seria extremamente eficiente computacionalmente, porém é de pouca utilidade prática para o cálculo de imagens acústicas, já que o número de 
direções amostradas geralmente é muito maior que o número de microfones e a restrição das localizações do nós restringe a geometria do arranjo.

Na definição da NFFT, assumimos posicionamento arbitrário dos nós $\mathbf{x}_{i}$ e restringimos os elementos de $I_{\mathbf{M}}$. No entanto, o caso em que ambos são arbitrários é interessante para algumas aplicações e é conhecido como NNFFT (fast Fourier transform for nonequispaced data in space and frequency domain). A definição de NNFFT, dada por [42, 8], é

$$
h_{i}=\sum_{m=0}^{M-1} \hat{h}_{m} e^{-j 2 \pi\left(\mathbf{v}_{m} \circ \mathbf{M}\right)^{T} \mathbf{x}_{i}}, \quad i=0,1, \ldots, N-1,
$$

em que o representa o produto de Schur-Hadamard, $\mathbf{x}_{i}, \mathbf{v}_{m} \in \mathbb{T}^{d}$ e $\mathbf{M}$ é o vetor de largura de banda. Seja $\mathcal{V} \triangleq\left\{\mathbf{v}_{m} \in \mathbb{T}^{d}: m=0,1, \ldots, M-1\right\}$. Note que, essencialmente, os vetores $\mathbf{k} \in I_{\mathbf{M}}$ foram substituídos por $\mathbf{v}_{m} \circ \mathbf{N}$. O fato da NNFFT não restringir a escolha dos elementos $\mathbf{v}_{\ell}$ implicará que a transformada será válida para uma amostragem arbitrária no espaço $U$, além de valer para geometrias arbitrárias. A complexidade computacional da NNFFT é da ordem de $\mathcal{O}\left(M \log M+|\log \epsilon|^{d}(M+N)\right)$, em que $\epsilon$ é a precisão dos cálculos, $d$ é a dimensão da transformada e o termo constante que multiplica essa ordem é diferente da NFFT. Para um tratamento dos algoritmos envolvidos na implementação da NNFFT, consultar $[43,44,45]$.

\subsection{Imagens acústicas por NFFT e NNFFT}

Vamos mostrar que os cálculos de imagens acústicas, tanto os métodos baseados nos sinais dos microfones quanto na matriz espectral, podem ser implementados por NFFT. Em geral, os algoritmos baseados na KAT são mais rápidos que os baseados em NFFT, porém requerem geometrias de arranjos e esquemas de amostragem mais restritos.

Seja a enumeração de direções $\mathbf{u}_{0}, \mathbf{u}_{1}, \ldots, \mathbf{u}_{M-1}$ arranjadas em uma grade retangular $M_{x} \times M_{y}\left(M_{x}, M_{y} \in 2 \mathbb{N}\right)$ tal que7

$$
\mathbf{u}_{m}=\mathbf{u}_{q+r M_{y}}=\left[\begin{array}{c}
u_{x}(q) \\
u_{y}(r) \\
\sqrt{1-u_{x}^{2}(q)-u_{y}^{2}(r)}
\end{array}\right],
$$

em que $-1 \leq u_{x}(q), u_{y}(r) \leq 1,0 \leq q<M_{x}, 0 \leq r<M_{y}$ e $M=M_{x} M_{y}$. Sejam os microfones do arranjo plano dispostos numa grade retangular nas posições $\mathbf{p}_{0}, \mathbf{p}_{1}, \ldots, \mathbf{p}_{N-1}$ tais que

$$
\mathbf{p}_{n}=\mathbf{p}_{\ell+i N_{y}}=\left[\begin{array}{c}
p_{x}(\ell) \\
p_{y}(i) \\
0
\end{array}\right]
$$

em que $0 \leq \ell<N_{x}, 0 \leq i<N_{y}$ e $N=N_{x} \times N_{y}$. 
Considere as equações do método X-DAS:

$$
\begin{aligned}
\mathbf{x} & =\mathbf{V} \mathbf{f} \\
\hat{\mathbf{f}} & =\frac{1}{N} \mathbf{V}^{H} \mathbf{x} .
\end{aligned}
$$

Expandindo a primeira equação, chegamos a

$$
\left[\begin{array}{c}
x_{0} \\
x_{1} \\
\vdots \\
x_{N-1}
\end{array}\right]=\left[\begin{array}{cccc}
e^{j \frac{\omega}{c} \mathbf{u}_{0}^{T} \mathbf{p}_{0}} & e^{j \frac{\omega}{c} \mathbf{u}_{1}^{T} \mathbf{p}_{0}} & \cdots & e^{j \frac{\omega}{c} \mathbf{u}_{M-1}^{T} \mathbf{p}_{0}} \\
e^{j \frac{\omega}{c} \mathbf{u}_{0}^{T} \mathbf{p}_{1}} & e^{j \frac{\omega}{c} \mathbf{u}_{1}^{T} \mathbf{p}_{1}} & \cdots & e^{j \frac{\omega}{c} \mathbf{u}_{M-1}^{T} \mathbf{p}_{1}} \\
\vdots & \vdots & & \vdots \\
e^{j \frac{\omega}{c} \mathbf{u}_{0}^{T} \mathbf{p}_{N-1}} & e^{j \frac{\omega}{c} \mathbf{u}_{1}^{T} \mathbf{p}_{N-1}} & \cdots & e^{j \frac{\omega}{c} \mathbf{u}_{M-1}^{T} \mathbf{p}_{N-1}}
\end{array}\right]\left[\begin{array}{c}
f_{0} \\
f_{1} \\
\vdots \\
f_{M-1}
\end{array}\right] .
$$

Note que a equação 5.12 possui estrutura parecida com a equação 5.4. Se pudermos especificar os nós $\mathbf{x}_{i}$ e as frequências $\mathbf{k}$, especificando consequentemente os conjuntos $\mathcal{X}$ e $I_{\mathbf{M}}$, usando os termos da matriz $\mathbf{V}$, poderemos obter imagens acústicas por NFFT.

Temos então

$$
\begin{aligned}
x_{n} & =\sum_{m=0}^{M-1} f_{m} e^{j \frac{\omega}{c} \mathbf{u}_{m}^{T} \mathbf{p}_{n}} \\
x_{n} & =\sum_{m=0}^{M-1} f_{m} \exp \left[j \frac{\omega}{c} u_{x}(m) p_{x}(n)+j \frac{\omega}{c} u_{y}(m) p_{y}(n)\right] .
\end{aligned}
$$

Como $n=n(\ell, y)=\ell+i N_{y}$ e $m=m(q, r)=q+r M_{y}$, podemos fazer

$$
x_{\ell, i}=\sum_{q, r} f_{q, r} \exp \left[j \frac{\omega}{c} u_{x}(q) p_{x}(\ell)+j \frac{\omega}{c} u_{y}(r) p_{y}(i)\right] .
$$

Escolhendo

$$
\begin{aligned}
& u_{x}(q)=\frac{2}{M_{x}}\left(-\frac{M_{x}}{2}+q\right)=-1+\frac{2 q}{M_{x}} \\
& u_{y}(r)=\frac{2}{M_{y}}\left(-\frac{M_{y}}{2}+r\right)=-1+\frac{2 r}{M_{y}}
\end{aligned}
$$

teremos amostragem uniforme no espaço $U$. Substituir essa amostragem do espaço $U$ na equação 5.15 leva a

$$
\begin{aligned}
x_{\ell, i}=\sum_{q, r} f_{q, r} \exp \left[-j 2 \pi\left(-\frac{\omega}{2 \pi c} \frac{2}{M_{x}} p_{x}(\ell)\right)\right. & \left(-\frac{M_{x}}{2}+q\right) \\
& \left.-j 2 \pi\left(-\frac{\omega}{2 \pi c} \frac{2}{M_{y}} p_{y}(i)\right)\left(-\frac{M_{y}}{2}+r\right)\right] .
\end{aligned}
$$

Note que os termos dentro da exponencial na equação 5.16 pertencem a $\mathcal{X}$ e $I_{\mathbf{M}}$ :

$$
\begin{array}{r}
{\left[\begin{array}{r}
-\frac{\omega}{2 \pi c} \frac{2}{M_{x}} p_{x}(\ell) \\
-\frac{\omega}{2 \pi c} \frac{2}{M_{y}} p_{y}(i)
\end{array}\right]} \\
{\left[\begin{array}{l}
-\frac{M_{x}}{2}+q \\
-\frac{M_{y}}{2}+r
\end{array}\right]}
\end{array}
$$


Portanto, se escolhermos

$$
\begin{aligned}
\mathcal{X} & =\left\{\mathbf{x}_{i}=-\frac{\omega}{\pi c}\left[\begin{array}{ll}
M_{x}^{-1} & M_{y}^{-1}
\end{array}\right] \circ \mathbf{p}_{i}: 0 \leq i<N\right\} \\
\mathbf{M} & =\left[\begin{array}{l}
M_{x} \\
M_{y}
\end{array}\right] \\
\mathbf{h} & =\mathbf{x}
\end{aligned}
$$

como entradas da biblioteca NFFT3 para o caso adjunto, teremos como saída o vetor $\hat{\mathrm{g}}=\mathbf{A}^{H} \mathbf{h}=N \mathbf{f}$. Ao dividir o vetor final por $N$, obtemos a mesma imagem acústica que seria obtida por X-DAS, porém realizando menos operações aritméticas. Note que o fato do arranjo ser plano fez com que o cálculo da imagem acústica fosse equivalente a uma NFFT 2D, isto é, $d=2$. Note também que não precisamos deduzir as equações para o caso adjunto, pois a biblioteca já o implementa automaticamente.

O método CSM-DAS, baseado na matriz espectral também pode ser implementado utilizando a NFTT. Considere a equação

$$
\begin{aligned}
& \mathbf{s}=\mathbf{A y} \\
& \mathbf{s}=\left[\begin{array}{llll}
\mathbf{v}_{\mathbf{u}_{0}}^{*} \otimes \mathbf{v}_{\mathbf{u}_{0}} & \mathbf{v}_{\mathbf{u}_{1}}^{*} \otimes \mathbf{v}_{\mathbf{u}_{1}} & \cdots & \mathbf{v}_{\mathbf{u}_{M-1}}^{*} \otimes \mathbf{v}_{\mathbf{u}_{M-1}}
\end{array}\right] \mathbf{y} \\
& {\left[\begin{array}{c}
s_{0} \\
s_{1} \\
\vdots \\
s_{N-1} \\
\vdots \\
s_{N^{2}-1}
\end{array}\right]=\left[\begin{array}{ccc}
e^{-j \omega \mathbf{u}_{0}^{T}\left(\mathbf{p}_{0}-\mathbf{p}_{0}\right) / c} & \cdots & e^{-j \omega \mathbf{u}_{M-1}^{T}\left(\mathbf{p}_{0}-\mathbf{p}_{0}\right) / c} \\
e^{-j \omega \mathbf{u}_{0}^{T}\left(\mathbf{p}_{0}-\mathbf{p}_{1}\right) / c} & \cdots & e^{-j \omega \mathbf{u}_{M-1}^{T}\left(\mathbf{p}_{0}-\mathbf{p}_{1}\right) / c} \\
\vdots & & \vdots \\
e^{-j \omega \mathbf{u}_{0}^{T}\left(\mathbf{p}_{0}-\mathbf{p}_{N-1}\right) / c} & \cdots & e^{-j \omega \mathbf{u}_{M-1}^{T}\left(\mathbf{p}_{0}-\mathbf{p}_{N-1}\right) / c} \\
\vdots & & \vdots \\
e^{-j \omega \mathbf{u}_{0}^{T}\left(\mathbf{p}_{N-1}-\mathbf{p}_{N-1}\right) / c} & \cdots & e^{-j \omega \mathbf{u}_{M-1}^{T}\left(\mathbf{p}_{N-1}-\mathbf{p}_{N-1}\right) / c}
\end{array}\right]\left[\begin{array}{c}
y_{0} \\
y_{1} \\
\vdots \\
y_{M-1}
\end{array}\right] .}
\end{aligned}
$$

Temos que

$$
\begin{aligned}
& s_{n}=\sum_{m=0}^{M-1} y_{m} e^{-j \omega \mathbf{u}_{m}^{T}\left(\mathbf{p}_{\lfloor n / N\rfloor}-\mathbf{p}_{\bmod (n, N)}\right) / c} \\
& s_{n}=\sum_{m=0}^{M-1} y_{m} \exp \left\{-j 2 \pi \frac{\omega}{2 \pi c} \mathbf{u}_{m}^{T}\left(\mathbf{p}_{\lfloor n / N\rfloor}-\mathbf{p}_{\bmod (n, N)}\right)\right\} \\
& s_{n}=\sum_{q, r} y_{q, r} \exp \left\{-j 2 \pi \frac{\omega}{2 \pi c}\left[\frac{2}{M_{x}}\left(-\frac{M_{x}}{2}+q\right) \frac{2}{M_{y}}\left(-\frac{M_{y}}{2}+r\right)\right]\left(\mathbf{p}_{\lfloor n / N\rfloor}-\mathbf{p}_{\bmod (n, N)}\right)\right\} \\
& s_{n}=\sum_{q, r} y_{q, r} \exp \left\{-j 2 \pi\left[\begin{array}{ll}
-\frac{M_{x}}{2}+q & -\frac{M_{y}}{2}+r
\end{array}\right]\right. \\
& \left.\left(\frac{\omega}{\pi c}\left[\begin{array}{ll}
M_{x}^{-1} & M_{y}^{-1}
\end{array}\right]^{T} \circ\left(\mathbf{p}_{\lfloor n / N\rfloor}-\mathbf{p}_{\bmod (n, N)}\right)\right)\right\} .
\end{aligned}
$$


Portanto basta escolher

$$
\begin{aligned}
& \mathcal{X}=\left\{\mathbf{x}_{i}=\frac{\omega}{\pi c}\left[\begin{array}{ll}
M_{x}^{-1} & M_{y}^{-1}
\end{array}\right]^{T} \circ\left(\mathbf{p}_{\lfloor i / N\rfloor}-\mathbf{p}_{\bmod (i, N)}\right): i=0,1, \ldots, N^{2}-1\right\} \\
& \mathbf{M}=\left[\begin{array}{l}
M_{x} \\
M_{y}
\end{array}\right] \\
& \mathbf{h}=\mathbf{s}
\end{aligned}
$$

que produziremos a mesma imagem acústica obtida por CSM-DAS, porém utilizando NFFT e com menor custo computacional. Note que a imagem acústica é novamente equivalente a uma NFFT 2D devido ao arranjo ser plano.

Podemos também deduzir a equação do método CSM-DAS com amostragem arbitrária no espaço $U$. Temos

$$
\begin{aligned}
s_{n}= & \sum_{m=0}^{M-1} y_{m} e^{-j \omega \mathbf{u}_{m}^{T}\left(\mathbf{p}_{\lfloor n / N\rfloor}-\mathbf{p}_{\bmod (n, N)}\right) / c} \\
s_{n}= & \sum_{q, r} y_{q, r} \exp \left\{-j 2 \pi\left(\left[\begin{array}{ll}
\frac{u_{x}(m)}{2} & \frac{u_{y}(m)}{2}
\end{array}\right] \circ\right.\right. \\
& {\left.\left[\begin{array}{ll}
M_{x} & M_{y}
\end{array}\right]\right) } \\
& \left.\left(\frac{\omega}{\pi c}\left[\begin{array}{ll}
M_{x}^{-1} & M_{y}^{-1}
\end{array}\right]^{T} \circ\left(\mathbf{p}_{\lfloor n / N\rfloor}-\mathbf{p}_{\bmod (n, N)}\right)\right)\right\},
\end{aligned}
$$

portanto basta escolher

$$
\begin{aligned}
\mathcal{X} & =\left\{\mathbf{x}_{i}=\frac{\omega}{\pi c}\left[\begin{array}{ll}
M_{x}^{-1} & M_{y}^{-1}
\end{array}\right]^{T} \circ\left(\mathbf{p}_{\lfloor i / N\rfloor}-\mathbf{p}_{\bmod (i, N)}\right): i=0,1, \ldots, N^{2}-1\right\} \\
\mathcal{V} & =\left\{\mathbf{v}_{m}=\left[\begin{array}{ll}
\frac{u_{x}(m)}{2} & \frac{u_{y}(m)}{2}
\end{array}\right]: m=0,1, \ldots, M-1\right\} \\
\mathbf{M} & =\left[\begin{array}{l}
M_{x} \\
M_{y}
\end{array}\right] \\
\mathbf{h} & =\mathbf{s}
\end{aligned}
$$

que obteremos a imagem acústica por NNFFT.

Apesar da NNFFT e da NFFT serem mais lentas que a KAT, porém mais rápidas que os métodos diretos por produto matricial, elas ainda são úteis pois podem ser aplicadas em casos nos quais a KAT não é aplicável. A NNFFT é uma transformada rápida para geometrias e amostragens arbitrárias; a NFFT é uma transformada rápida para geometrias de arranjo arbitrárias; e a KAT somente pode ser aplicada ao casos de amostragem separável e geometria separável.

\subsection{Transformada de arranjo de Kronecker}

Veremos a seguir que as matrizes $\mathbf{A}$ e $\mathbf{V}$, envolvidas no problema de estimação de imagens acústicas, apresentam uma certa estrutura quando escolhemos uma amostragem 
separável do espaço de possíveis direções das fontes e quando usamos um arranjo com geometria separável. Uma vez satisfeitas essas duas hipóteses, as matrizes A e V podem ser decompostas em produtos de Kronecker de matrizes menores. Essas matrizes da decomposição possuem dimensão bem menor que as matrizes originais, tendo em vista a própria definição do produto de Kronecker apresentada anteriormente, daí a grande redução do custo computacional e de memória.

Considere um arranjo plano com $N$ microfones dispostos em uma grade retangular $N_{x} \times N_{y}$ localizados nas coordenadas $\mathbf{p}_{0}, \mathbf{p}_{1}, \ldots, \mathbf{p}_{N-1} \in \mathbb{R}^{3}$ dadas por

$$
\mathbf{p}_{n}=\mathbf{p}_{\ell+i N_{y}}=\left[\begin{array}{c}
p_{x}(\ell) \\
p_{y}(i) \\
0
\end{array}\right]
$$

em que $0 \leq \ell<N_{x}, 0 \leq i<N_{y}$.

As direções de visada $\mathbf{u}_{0}, \mathbf{u}_{1}, \ldots, \mathbf{u}_{M-1}$ são escolhidas na semiesfera superior e são mapeadas para uma grade retangular $M_{x} \times M_{y}$ tal que

$$
\mathbf{u}_{m}=\mathbf{u}_{q+r M_{y}}=\left[\begin{array}{c}
u_{x}(q) \\
u_{y}(r) \\
\sqrt{1-u_{x}^{2}(q)-u_{y}^{2}(r)}
\end{array}\right]
$$

em que $-1 \leq u_{x}(q), u_{y}(r) \leq 1,0 \leq q<M_{x}, 0 \leq r<M_{y}$.

Quando os sensores do arranjo estão posicionados de acordo com a equação (5.38) (caso este no qual diz-se que o arranjo possui geometria separável) e as direções de visada também são escolhidas numa grade retangular, como na equação (5.39), a matriz $\mathbf{V}$ pode ser escrita como o produto de Kronecker de duas matrizes menores.

O elemento $(n, m)$ de $\mathbf{V}$ para uma frequência $\omega_{k}$ é

$$
[\mathbf{V}]_{n, m}=e^{j \frac{\omega_{k}}{c} \mathbf{u}_{m}^{T} \mathbf{p}_{n}}=[\mathbf{V}]_{\ell+i N_{y}, q+r M_{y}}=e^{j \omega_{k} u_{x}(q) p_{x}(\ell) / c} e^{j \omega_{k} u_{y}(r) p_{y}(i) / c}
$$

Se definirmos duas matrizes diretoras $\mathbf{V}_{x}$ e $\mathbf{V}_{y}$ com elementos

$$
\begin{aligned}
& {\left[\mathbf{V}_{x}\right]_{\ell, q}=e^{j \omega_{k} u_{x}(q) p_{x}(\ell) / c}} \\
& {\left[\mathbf{V}_{y}\right]_{i, r}=e^{j \omega_{k} u_{y}(r) p_{y}(i) / c},}
\end{aligned}
$$


teremos

$$
\begin{aligned}
\mathbf{V}_{x} & =\left[\begin{array}{llll}
\mathbf{v}_{x}\left(u_{x}(0)\right) & \mathbf{v}_{x}\left(u_{x}(1)\right) & \cdots & \mathbf{v}_{x}\left(u_{x}\left(M_{x}-1\right)\right)
\end{array}\right] \\
& =\left[\begin{array}{ccc}
e^{j \omega_{k}(-1) p_{x}(0) / c} & \cdots & e^{j \omega_{k}(1) p_{x}(0) / c} \\
e^{j \omega_{k}(-1) p_{x}(1) / c} & \cdots & e^{j \omega_{k}(1) p_{x}(1) / c} \\
\vdots & & \vdots \\
e^{j \omega_{k}(-1) p_{x}\left(N_{x}-1\right) / c} & \cdots & e^{j \omega_{k}(1) p_{x}\left(N_{x}-1\right) / c}
\end{array}\right] \\
\mathbf{V}_{y} & =\left[\begin{array}{llll}
\mathbf{v}_{y}\left(u_{y}(0)\right) & \mathbf{v}_{y}\left(u_{y}(1)\right) & \cdots & \mathbf{v}_{y}\left(u_{y}\left(M_{x}-1\right)\right)
\end{array}\right] \\
& =\left[\begin{array}{ccc}
e^{j \omega_{k}(-1) p_{y}(0) / c} & \cdots & e^{j \omega_{k}(1) p_{y}(0) / c} \\
e^{j \omega_{k}(-1) p_{y}(1) / c} & \cdots & e^{j \omega_{k}(1) p_{y}(1) / c} \\
\vdots & & \vdots \\
e^{j \omega_{k}(-1) p_{y}\left(N_{y}-1\right) / c} & \cdots & e^{j \omega_{k}(1) p_{y}\left(N_{y}-1\right) / c}
\end{array}\right],
\end{aligned}
$$

em que $\mathbf{V}_{x}$ possui dimensões $N_{x} \times M_{x}$ e a matriz $\mathbf{V}_{y}$ possui dimensões $N_{y} \times M_{y}$.

Nessas condições, é fácil verificar que

$$
\mathbf{V}=\mathbf{V}_{x} \otimes \mathbf{V}_{y}
$$

e os produtos matriciais $\mathbf{V} \mathbf{x}, \mathbf{V}^{H} \mathbf{x}, \mathbf{A y}, \mathbf{A}^{H} \mathbf{s}, \mathbf{A}^{H} \mathbf{A y}$, etc podem ser calculados de forma mais eficiente [4].

Não só a matriz $\mathbf{V}$ pode ser escrita como um produto de Kronecker no caso de geometria e amostragem separáveis, mas também o próprio vetor diretor do arranjo possui essa propriedade:

$$
\mathbf{v}\left(\mathbf{u}_{m}\right)=\mathbf{v}\left(\mathbf{u}_{q+r M_{y}}\right)=\mathbf{v}_{x}\left(u_{x}(q)\right) \otimes \mathbf{v}_{y}\left(u_{y}(r)\right)
$$

em que

$$
\begin{aligned}
& \mathbf{v}_{x}\left(u_{x}(q)\right) \triangleq\left[\begin{array}{c}
e^{j \omega_{k} u_{x}(q) p_{x}(0) / c} \\
e^{j \omega_{k} u_{x}(q) p_{x}(1) / c} \\
\vdots \\
e^{j \omega u_{x}(q) p_{x}\left(N_{x}-1\right) / c}
\end{array}\right], \\
& \mathbf{v}_{y}\left(u_{y}(q)\right) \triangleq\left[\begin{array}{c}
e^{j \omega_{k} u_{y}(q) p_{y}(0) / c} \\
e^{j \omega_{k} u_{y}(q) p_{y}(1) / c} \\
\vdots \\
e^{j \omega u_{y}(q) p_{y}\left(N_{y}-1\right) / c}
\end{array}\right] .
\end{aligned}
$$

A equação (5.44) é a própria definição de arranjos separáveis. Portanto, quando dizemos que um arranjo é separável, queremos dizer que seu vetor diretor obedece a esta propriedade [16]. 

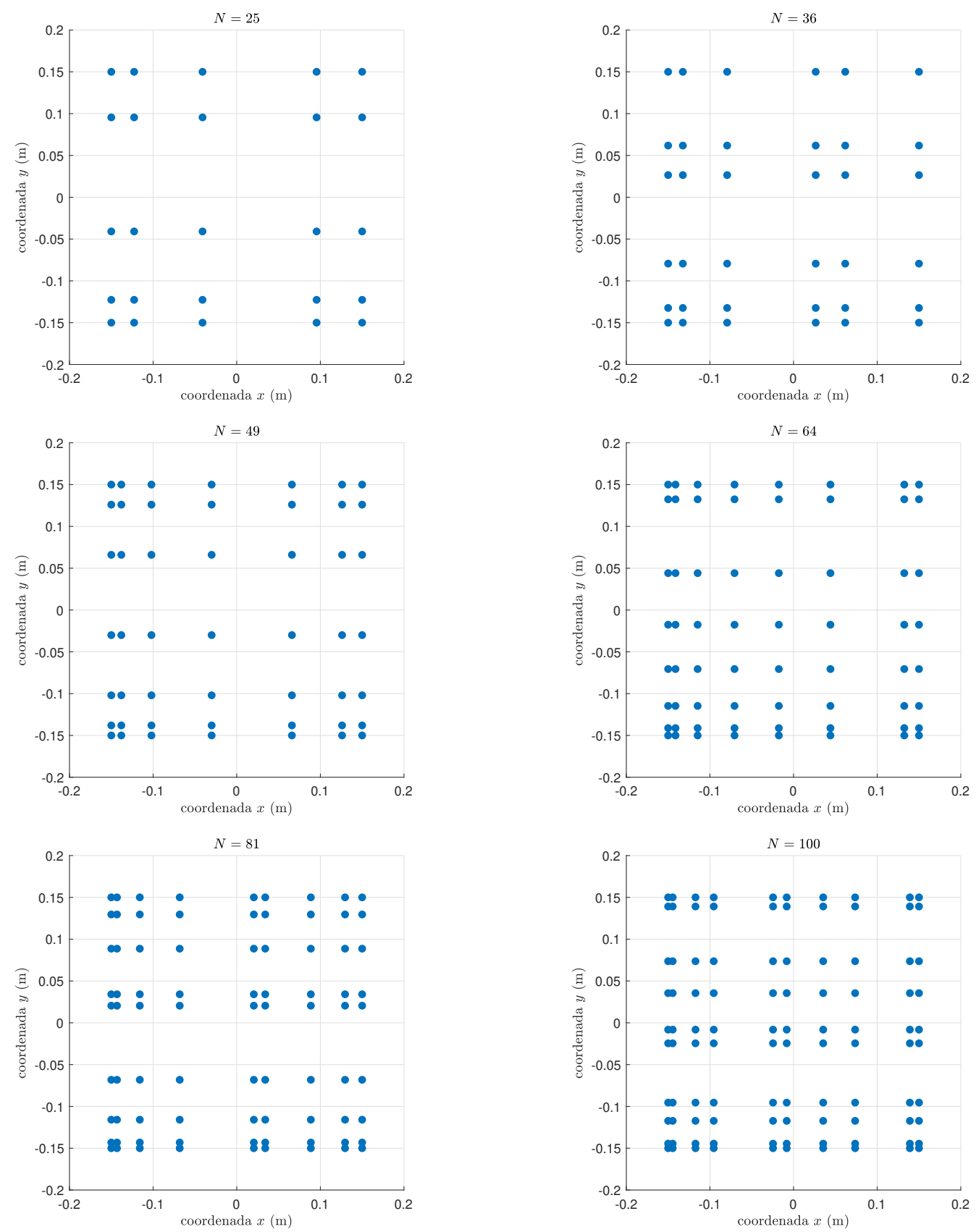

Figura 8 - Geometrias de arranjos separáveis compostas de subarranjos lineares não uniformes e não redundantes. Cada ponto representa a posição de um microfone.

A Figura 8 mostra exemplos de arranjos planos com geometria separável em que cada subarrajo linear é não-redundante (NRA) com um mínimo de diferenças ausentes (minimum missing lags). A geometria NRA é usada pois é capaz de conseguir a maior resolução para uma dada quantidade de elementos [46] e possui melhor performance em problemas de reconstrução esparsa [47]. Além disso, a escolha de uma geometria nãouniforme elimina a restrição de frequência máxima de operação de arranjos uniformes limitada pelo teorema de Nyquist [8]. 
Como mostrado anteriormente, o beamformer DAS é equivalente a

$$
\hat{\mathbf{f}}=\frac{1}{N} \mathbf{V}^{H} \mathbf{x}
$$

Ao aplicar a decomposição da matriz $\mathbf{V}=\mathbf{V}_{x} \otimes \mathbf{V}_{y}$, temos

$$
\hat{\mathbf{f}}=\frac{1}{N}\left(\mathbf{V}_{x} \otimes \mathbf{V}_{y}\right)^{H} \mathbf{x}
$$

Aplicar a identidade

$$
\operatorname{vec}\left\{\mathbf{B Q A}^{T}\right\}=(\mathbf{A} \otimes \mathbf{B}) \operatorname{vec}\{\mathbf{Q}\}
$$

leva a

$$
\hat{\mathbf{F}}=\mathbf{V}_{y}^{H} \mathbf{X} \mathbf{V}_{x}^{*}
$$

em que $\hat{\mathbf{f}}=\operatorname{vec}\{\hat{\mathbf{F}}\}$ e $\mathbf{x}=\operatorname{vec}\{\mathbf{X}\}$. Podemos também obter uma estimativa de $\mathbf{y}$ calculando a equação (5.46) e elevando ao quadrado o módulo dos termos do vetor $\hat{\mathbf{f}}$. A equação (5.49) é chamada KAT adjunta.

Em alguns algoritmos, como no caso de compressive beamforming, também é necessário calcular produtos da forma $\mathbf{x}=\mathbf{V f}$. Aplicar a decomposição de $\mathbf{V}$ leva a

$$
\mathbf{x}=\left(\mathbf{V}_{x} \otimes \mathbf{V}_{y}\right) \mathbf{f}
$$

Ao aplicar a identidade (5.48), temos

$$
\mathbf{X}=\mathbf{V}_{y} \mathbf{F} \mathbf{V}_{x}^{T}
$$

A equação (5.51) é chamada KAT direta.

Pode-se, ainda, combinar a KAT direta com a KAT adjunta para calcular produtos da forma $\hat{\mathbf{f}}=\mathbf{V}^{H} \mathbf{V f}$ e formar a chamada KAT direta-adjunta. Temos

$$
\hat{\mathbf{f}}=\left[\left(\mathbf{V}_{x} \otimes \mathbf{V}_{y}\right)^{H}\left(\mathbf{V}_{x} \otimes \mathbf{V}_{y}\right)\right] \mathbf{f} .
$$

Aplicamos a identidade

$$
(\mathbf{A} \otimes \mathbf{B})(\mathbf{C} \otimes \mathbf{D})=\mathbf{A C} \otimes \mathbf{B D}
$$

e chegamos em

$$
\hat{\mathbf{f}}=\left[\left(\mathbf{V}_{x}^{H} \mathbf{V}_{x}\right) \otimes\left(\mathbf{V}_{y}^{H} \mathbf{V}_{y}\right)\right] \mathbf{f} .
$$

Por fim, aplicamos a identidade (5.48) resultando em

$$
\hat{\mathbf{F}}=\mathbf{V}_{y}^{H} \mathbf{V}_{y} \mathbf{F} \mathbf{V}_{x}^{T} \mathbf{V}_{x}^{*}
$$

As três versões da KAT (direta, adjunta e direta-adjunta) deduzidas até agora são aplicáveis a problemas que envolvem o uso do vetor $\mathbf{x}$ diretamente, no entanto vários algoritmos para estimar imagens acústicas trabalham com a matriz espectral do arranjo, 
definida anteriormente como $\mathbf{S}_{\mathbf{x}}=E\left\{\mathbf{x x}^{H}\right\}$. Portanto definiremos a seguir versões da KAT que utilizam a matriz espectral.

Considere agora a equação (4.16) repetida a seguir:

$$
\mathbf{S}_{\mathbf{x}}\left(\omega_{k}\right)=\mathbf{V}\left(\omega_{k}\right) E\left\{\mathbf{f}\left(\omega_{k}\right) \mathbf{f}^{H}\left(\omega_{k}\right)\right\} \mathbf{V}^{H}\left(\omega_{k}\right)+\sigma^{2} \mathbf{I}
$$

Definir $\mathbf{S}_{\mathbf{f}}=E\left\{\mathbf{f}\left(\omega_{k}\right) \mathbf{f}^{H}\left(\omega_{k}\right)\right\}$ como a matriz espectral de $\mathbf{f}$ e ignorar o ruído leva a

$$
\mathbf{S}_{\mathbf{x}}=\mathbf{V S}_{\mathbf{f}} \mathbf{V}^{H}
$$

Como assumimos anteriormente, as fontes são descorrelacionadas e, consequentemente, $\mathbf{S}_{\mathbf{f}}$ é uma matriz diagonal. Pode-se, então, aplicar a identidade proposta por [48], isto é,

$$
\operatorname{vec}\left\{\mathbf{B P A}^{T}\right\}=(\mathbf{A} \odot \mathbf{B}) \operatorname{vecd}\{\mathbf{P}\}
$$

em que $\mathbf{P}$ é uma matriz diagonal e vecd $\{\mathbf{A}\}$ é um operador que retorna os elementos da diagonal de uma matriz quadrada qualquer A como um vetor coluna.

Aplicar a identidade (5.57) à equação (5.56) leva a

$$
\operatorname{vec}\left\{\mathbf{S}_{\mathbf{x}}\right\}=\left(\mathbf{V}^{*} \odot \mathbf{V}\right) \operatorname{vecd}\left\{\mathbf{S}_{\mathbf{f}}\right\}
$$

Note que os elementos da diagonal de $\mathbf{S}_{\mathbf{f}}$ correspondem aos valores dos pixels da imagem acústica, conforme discutido anteriormente, $\mathbf{y}=\operatorname{vec}\{\mathbf{Y}\}=\operatorname{vecd}\left\{\mathbf{S}_{\mathbf{f}}\right\}, \mathbf{A}=\left(\mathbf{V}^{*} \odot \mathbf{V}\right)$ e $\mathbf{s}=\operatorname{vec}\left\{\mathbf{S}_{\mathbf{x}}\right\}$. Como $\mathbf{V}=\mathbf{V}_{x} \otimes \mathbf{V}_{y}$, então

$$
\mathbf{A}=\mathbf{V}^{*} \odot \mathbf{V}=\left(\mathbf{V}_{x} \otimes \mathbf{V}_{y}\right)^{*} \odot\left(\mathbf{V}_{x} \otimes \mathbf{V}_{y}\right)
$$

O teorema a seguir permite reescrever a equação (5.59) [6]:

Teorema 1 Sejam as matrizes de bloco compativeis

$$
\begin{aligned}
\mathbf{A} & =\left[\begin{array}{llll}
\mathbf{a}_{1} & \mathbf{a}_{2} & \cdots & \mathbf{a}_{p}
\end{array}\right] \\
\mathbf{B} & =\left[\begin{array}{llll}
\mathbf{b}_{1} & \mathbf{b}_{2} & \cdots & \mathbf{b}_{q}
\end{array}\right] \\
\mathbf{C} & =\left[\begin{array}{llll}
\mathbf{c}_{1} & \mathbf{c}_{2} & \cdots & \mathbf{c}_{p}
\end{array}\right] \\
\mathbf{D} & =\left[\begin{array}{llll}
\mathbf{d}_{1} & \mathbf{d}_{2} & \cdots & \mathbf{d}_{q}
\end{array}\right],
\end{aligned}
$$

então

$$
(\mathbf{A} \otimes \mathbf{B}) \odot(\mathbf{C} \otimes \mathbf{D})=\Xi[(\mathbf{A} \odot \mathbf{C}) \otimes(\mathbf{B} \odot \mathbf{D})]
$$

em que $\Xi$ é uma matriz de permutação. 
A matriz de permutação $\Xi$ é obtida observando a seguinte propriedade de permutação do produto de Kronecker [49]

$$
\mathbf{P}_{m, n}(\mathbf{a} \otimes \mathbf{b})=(\mathbf{b} \otimes \mathbf{a}),
$$

em que a, b são vetores coluna de dimensões $m \times 1$ e $n \times 1$, respectivamente; $\mathbf{P}_{m, n}$ é uma matriz quadrada de tamanho $m n \times m n$ composta por um arranjo de $n \times m$ blocos $\mathbf{S}_{i, j}$ de dimensão $m \times n$ tal que o elemento $\left[\mathbf{S}_{i, j}\right]_{j, i}=1$ e todos os outros elementos do mesmo bloco são iguais a zero.

Da prova da identidade (5.61), mostrada em [6], temos que

$$
\Xi=\mathbf{I}_{N_{x}} \otimes \mathbf{P}_{N_{y}, N_{x}}^{T} \otimes \mathbf{I}_{N_{y}}
$$

em que $\mathbf{I}_{N_{x}}$ e $\mathbf{I}_{N_{y}}$ são matrizes identidade. Note que a dimensão da matriz de permutação $\mathbf{P}_{N_{y}, N_{x}}$ é $N_{x} N_{y} \times N_{x} N_{y}$, ou seja, depende apenas do número de microfones.

Ao aplicar a relação (5.61) à equação (5.59), temos

$$
\mathbf{A}=\left(\mathbf{V}_{x} \otimes \mathbf{V}_{y}\right)^{*} \odot\left(\mathbf{V}_{x} \otimes \mathbf{V}_{y}\right)=\Xi\left[\left(\mathbf{V}_{x}^{*} \odot \mathbf{V}_{x}\right) \otimes\left(\mathbf{V}_{y}^{*} \odot \mathbf{V}_{y}\right)\right]=\Xi\left(\tilde{\mathbf{V}}_{x} \otimes \tilde{\mathbf{V}}_{y}\right)
$$

em que $\tilde{\mathbf{V}}_{x} \triangleq\left(\mathbf{V}_{x}^{*} \odot \mathbf{V}_{x}\right)$ e $\tilde{\mathbf{V}}_{y} \triangleq\left(\mathbf{V}_{y}^{*} \odot \mathbf{V}_{y}\right)$.

Temos

$$
\operatorname{vec}\left\{\mathbf{S}_{\mathbf{x}}\right\}=\mathbf{\Xi}\left(\tilde{\mathbf{V}}_{x} \otimes \tilde{\mathbf{V}}_{y}\right) \operatorname{vecd}\left\{\mathbf{S}_{\mathbf{f}}\right\}
$$

Definimos

$$
\operatorname{vec}\{\mathbf{Z}\}=\left(\tilde{\mathbf{V}}_{x} \otimes \tilde{\mathbf{V}}_{y}\right) \operatorname{vecd}\left\{\mathbf{S}_{\mathbf{f}}\right\} .
$$

Aplicar a identidade (5.48) leva a

$$
\mathbf{Z}=\tilde{\mathbf{V}}_{y} \mathbf{Y} \tilde{\mathbf{V}}_{x}^{T}, \quad \operatorname{vec}\left\{\mathbf{S}_{\mathbf{x}}\right\}=\boldsymbol{\Xi} \operatorname{vec}\{\mathbf{Z}\}
$$

Essa equação define a chamada CSM KAT direta.

Para calcular expressões da forma $\hat{\mathbf{y}}=\mathbf{A}^{H} \mathbf{s}$, utilizamos a CSM KAT adjunta. Temos

$$
\operatorname{vec}\{\hat{\mathbf{Y}}\}=\left[\Xi\left(\tilde{\mathbf{V}}_{x} \otimes \tilde{\mathbf{V}}_{y}\right)\right]^{H} \operatorname{vec}\left\{\mathbf{S}_{\mathbf{x}}\right\}=\left(\tilde{\mathbf{V}}_{x}^{H} \otimes \tilde{\mathbf{V}}_{y}^{H}\right) \boldsymbol{\Xi}^{T} \operatorname{vec}\left\{\mathbf{S}_{\mathbf{x}}\right\} .
$$

Definimos

$$
\operatorname{vec}\{\mathbf{Z}\}=\mathbf{\Xi}^{T} \operatorname{vec}\left\{\mathbf{S}_{\mathbf{x}}\right\}
$$

e aplicamos a identidade (5.48) para obter

$$
\operatorname{vec}\{\mathbf{Z}\}=\mathbf{\Xi}^{T} \operatorname{vec}\left\{\mathbf{S}_{\mathbf{x}}\right\}, \quad \mathbf{Y}=\tilde{\mathbf{V}}_{y}^{H} \mathbf{Z} \tilde{\mathbf{V}}_{x}^{*}
$$

A combinação da CSM KAT direta com a adjunta produzirá a CSM KAT diretaadjunta, usada para acelerar os cálculos de expressões da forma $\hat{\mathbf{y}}=\mathbf{A}^{H} \mathbf{A y}$. Temos

$$
\operatorname{vec}\{\hat{\mathbf{Y}}\}=\left(\tilde{\mathbf{V}}_{x}^{H} \otimes \tilde{\mathbf{V}}_{y}^{H}\right) \Xi^{T} \Xi\left(\tilde{\mathbf{V}}_{x} \otimes \tilde{\mathbf{V}}_{y}\right) \operatorname{vec}\{\mathbf{Y}\}
$$


Como a matriz de permutação é ortogonal, $\Xi^{T} \Xi$ é igual a matriz identidade, portanto

$$
\hat{\mathbf{Y}}=\tilde{\mathbf{V}}_{y}^{H} \tilde{\mathbf{V}}_{y} \mathbf{Y} \tilde{\mathbf{V}}_{x}^{T} \tilde{\mathbf{V}}_{x}^{*}
$$

Como os elementos de $\mathbf{V}_{x}$ e $\mathbf{V}_{y}$ são números complexos de módulo igual a 1 (veja equação (5.45)), a NFFT e a NNFFT podem ser usadas para acelerar ainda mais os produtos nas equações (5.70) e (5.72). No entanto, o número de microfones no arranjo e o número de direções de visada deve ser grande o suficiente para causar algum ganho [4].

\subsubsection{DAS acelerado por KAT}

Como mostrado anteriormente, existem duas implementações principais de DAS: a baseada na matriz espectral e a da equação (5.46). Na seção 5.3, mostrou-se que ambas as implementações podem ser aceleradas pela KAT.

Utilizando as propriedades do produto de Kronecker, mostraremos que a formulação baseada na forma vetorizada da matriz espectral s denominada CSM-DAS é dada por

$$
\mathbf{y}=\frac{1}{N^{2}} \mathbf{A}^{H} \mathbf{s}
$$

Partindo da equação (4.33), temos que

$$
\begin{aligned}
\hat{y}\left(\mathbf{u}_{m}\right) & =\frac{1}{N^{2}} \mathbf{v}^{H}\left(\mathbf{u}_{m}\right) \mathbf{S v}\left(\mathbf{u}_{m}\right) \\
& =\frac{1}{N^{2}}\left(\mathbf{v}^{T}\left(\mathbf{u}_{m}\right) \otimes \mathbf{v}^{H}\left(\mathbf{u}_{m}\right)\right) \operatorname{vec}\{\mathbf{S}\},
\end{aligned}
$$

em que usamos a identidade (5.48). Como $(\mathbf{A} \otimes \mathbf{B})^{H}=\mathbf{A}^{H} \otimes \mathbf{B}^{H}$, então

$$
\begin{aligned}
\hat{y}\left(\mathbf{u}_{m}\right) & =\frac{1}{N^{2}}\left(\mathbf{v}^{*}\left(\mathbf{u}_{m}\right) \otimes \mathbf{v}\left(\mathbf{u}_{m}\right)\right)^{H} \mathbf{s} \\
& =\frac{1}{N^{2}} \mathbf{a}_{m}^{H} \mathbf{s}
\end{aligned}
$$

em que $\mathbf{a}_{m}$ é a $m$-ésima coluna de $\mathbf{A}$. Logo, basta escrever a equação anterior em forma matricial para y que concluímos a demonstração.

Já na formulação de X-DAS, da equação (5.46), dada por

$$
\mathbf{f}=\frac{1}{N} \mathbf{V}^{H} \mathbf{x}
$$

é importante lembrar que o vetor $\mathbf{y}$, a imagem acústica em si, pode ser obtido facilmente de $\mathbf{f}$ no método X-DAS.

De acordo com a seção 5.3, denominaremos X-KAT a versão acelerada correspondente ao método X-DAS, dada pela equação (5.49):

$$
\mathbf{F}=\mathbf{V}_{y}^{H} \mathbf{X} \mathbf{V}_{x}^{*}
$$


Denominaremos CSM-KAT a versão acelerada correspondente ao método CSM-DAS, dada pela equação (5.70):

$$
\operatorname{vec}(\mathbf{Z})=\mathbf{\Xi}^{T} \mathbf{s}, \quad \mathbf{Y}=\tilde{\mathbf{V}}_{y}^{H} \mathbf{Z} \tilde{\mathbf{V}}_{x}^{*}
$$

em que $\Xi^{T}$ é um matriz de permutação.

Como $\mathbf{V}^{H}$ possui dimensões $M_{x} M_{y} \times N_{x} N_{y}$ e $\mathbf{x}$ possui dimensões $N_{x} N_{y} \times 1$, então o cálculo de uma imagem acústica utilizando X-DAS requer $M_{x} M_{y} N_{x} N_{y}$ MACs. Por outro lado, $\mathbf{V}_{y}^{H}$ é $M_{y} \times N_{y}, \mathbf{V}_{x}^{*}$ é $N_{x} \times N_{x}$ e $\mathbf{X}$ é $N_{y} \times N_{x}$; portanto o cálculo de uma imagem utilizando X-KAT requer $N_{y} N_{x} M_{y}+N_{x} M_{y} M_{x}$ MACs se os produtos forem executados da esquerda para a direita ou $N_{y} M_{x} M_{y}+N_{x} N_{y} M_{x}$ se os produtos forem executados da direita para a esquerda. Se escolhermos $M_{x}=M_{y}=\sqrt{M}$ (imagem quadrada) e $N_{y}=N_{x}=\sqrt{N}$, a aceleração relativa, em termos de número de MACs, é de

$$
\frac{M_{x} M_{y} N_{x} N_{y}}{N_{y} N_{x} M_{y}+N_{x} M_{y} M_{x}}=\frac{M N}{N \sqrt{M}+M \sqrt{N}} \approx \frac{M N}{M \sqrt{N}}=\sqrt{N},
$$

em que a aproximação decorre de o número de pixels da imagem ser geralmente muito maior que o número de microfones, isto é, $M \gg N$.

A Figura 9 mostra a aceleração relativa do tempo de cálculo de uma mesma imagem acústica utilizando X-DAS e X-KAT. Cada ponto corresponde a uma média de 100 realizações de uma distribuição com duas fontes na frequência de $6000 \mathrm{~Hz}$ e um arranjo separável com $N=64$ microfones.

Note que os ganhos no tempo de execução são maiores que $\sqrt{N}$ devido a vários fatores, dentre eles: alocação de memória ao criar uma variável que salva os resultados do cálculo na memória; arquiteturas modernas empregam unidades aritméticas paralelas; em um dos casos se aloca uma quantidade muito menor de memória do que no outro, como será mostrado na seção 5.3.3. Portanto a aceleração relativa em termos de operações MACs serve apenas como uma aproximação do custo computacional e depende do hardware específico utilizado.

A matriz $\mathbf{A}^{H}$ possui dimensões $M_{x} M_{y} \times N_{x}^{2} N_{y}^{2}$ e o vetor $\mathbf{s}$ possui dimensões $N_{x}^{2} N_{y}^{2} \times 1$, então o cálculo utilizando CSM-DAS requer $M_{x} M_{y} N_{x}^{2} N_{y}^{2}$ MACs. A matriz $\tilde{\mathbf{V}}_{y}^{H}$ é $M_{y} \times N_{y}^{2}$, a matriz $\tilde{\mathbf{V}}_{x}^{*}$ é $N_{y}^{2} \times M_{y}$ e a matriz $\mathbf{Z}$ é $N_{y}^{2} \times N_{x}^{2}$; portanto o cálculo utilizando CSM-DAS requer $M_{y} N_{y}^{2} N_{x}^{2}+M_{y} N_{x}^{2} M_{x}$ MACs, se os produtos forem executados da esquerda para a direita, e $N_{y}^{2} N_{x}^{2} M_{x}+M_{y} N_{y}^{2} M_{x}$ MACs, se os produtos forem executados da direita para esquerda. Considerando novamente que $N_{x}=N_{y}=\sqrt{N}$ e $M_{x}=M_{y}=\sqrt{N}$, teremos uma aceleração relativa, em número de MACs, da ordem de

$$
\frac{M_{x} M_{y} N_{x}^{2} N_{y}^{2}}{M_{y} N_{y}^{2} N_{x}^{2}+M_{y} N_{x}^{2} M_{x}}=\frac{M N^{2}}{\sqrt{M} N^{2}+M N} \approx \frac{M N^{2}}{M N}=N,
$$

em que a hipótese $M \gg N$ é tida como verdadeira. 


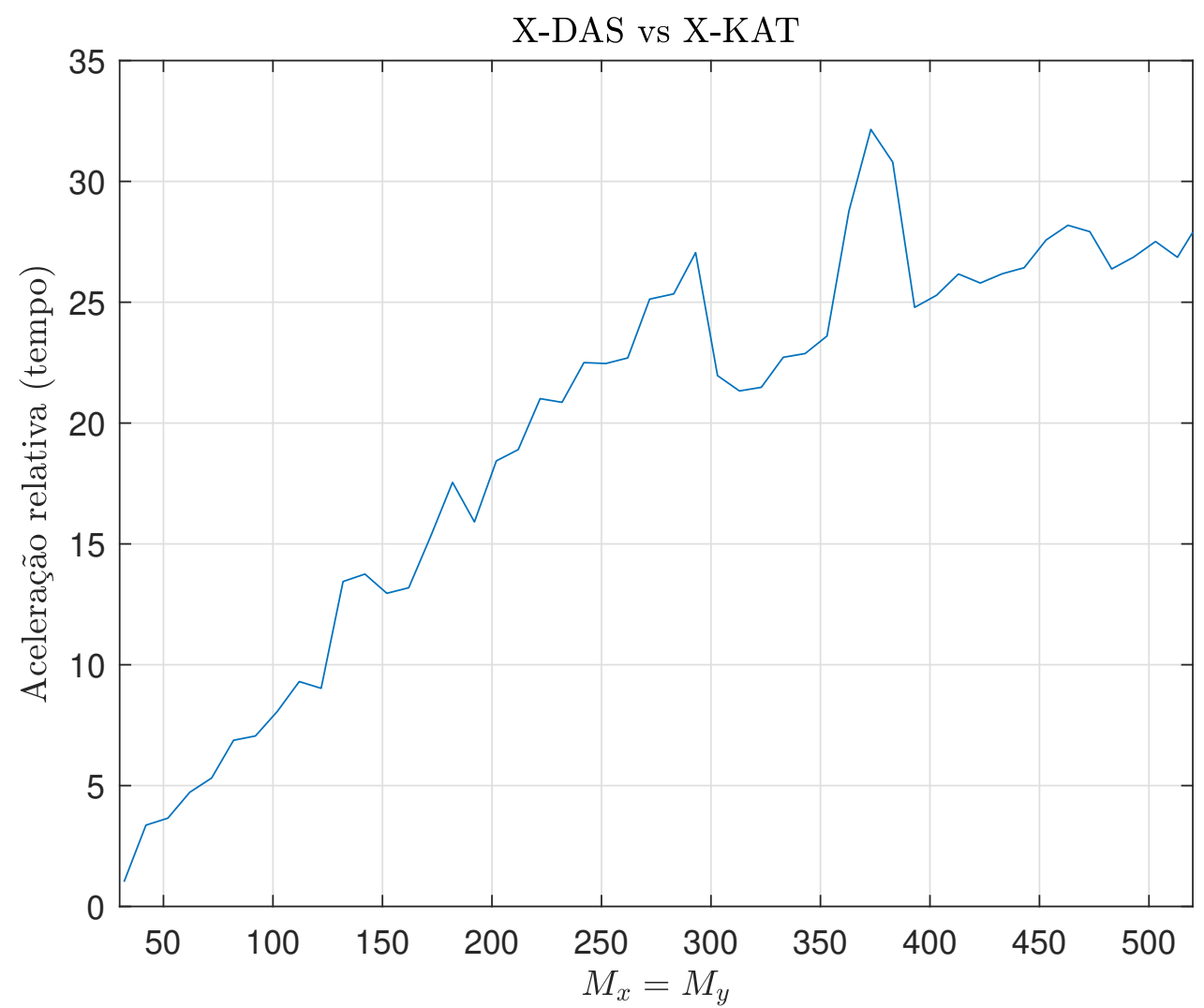

Figura 9 - Aceleração média relativa do tempo de cálculo de X-KAT em relação a X-DAS para uma distribuição com três fontes pontuais e $N=64$.

A Figura 10 mostra a aceleração relativa do tempo de cálculo de uma mesma imagem acústica utilizando CSM-DAS, CSM-KAT e NFFT baseada nas equações de CSM. A NFFT realmente reduz o custo computacional do método por produto matricial direto (CSM-DAS), porém a KAT apresenta um desempenho superior.

Note que a aceleração relativa entre X-DAS e X-KAT é aproximadamente de ordem $\sqrt{N}$ e a aceleração relativa entre CSM-DAS e CSM-KAT é aproximadamente de ordem $N$. Comparando os dois métodos acelerados, temos que a aceleração relativa entre X-KAT e CSM-KAT é da ordem de

$$
\frac{\sqrt{M} N^{2}+M N}{N \sqrt{M}+M \sqrt{N}} \approx \frac{M N}{M \sqrt{N}}=\frac{N}{\sqrt{N}}=\sqrt{N}
$$

isto é, X-KAT requer aproximadamente $\sqrt{N}$ vezes menos MACs que CSM-KAT. Esse fato poderia levar à conclusão de que é mais vantajoso utilizar CSM-KAT, porém os dois algoritmos são relativamente diferentes: um utiliza a matriz espectral e o outro trabalha diretamente com as amostras nos microfones. No entanto, a X-KAT estima valores complexos e não necessita da hipótese de fontes descorrelacionadas, porém pode ser usada para reconstruir sinais no tempo e vai além do imageamento de intensidade sonora; enquanto a CSM-KAT estima valores reais, requer fontes descorrelacionadas e não permite a reconstrução do sinal. 


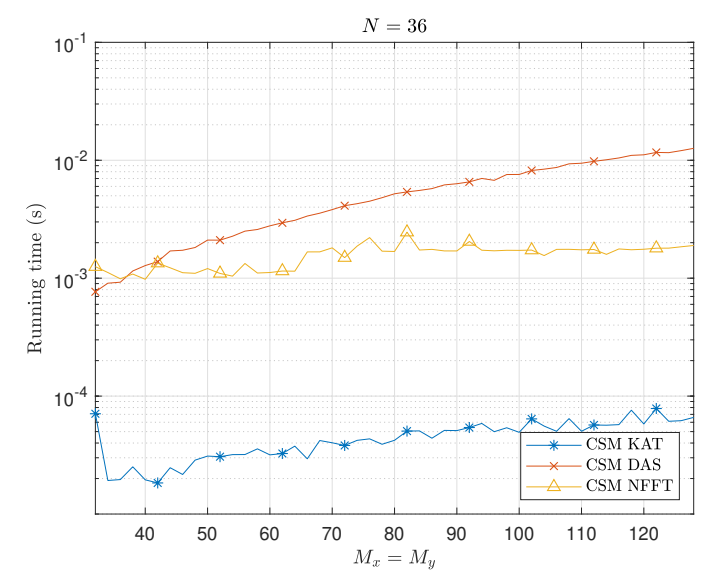

(a)

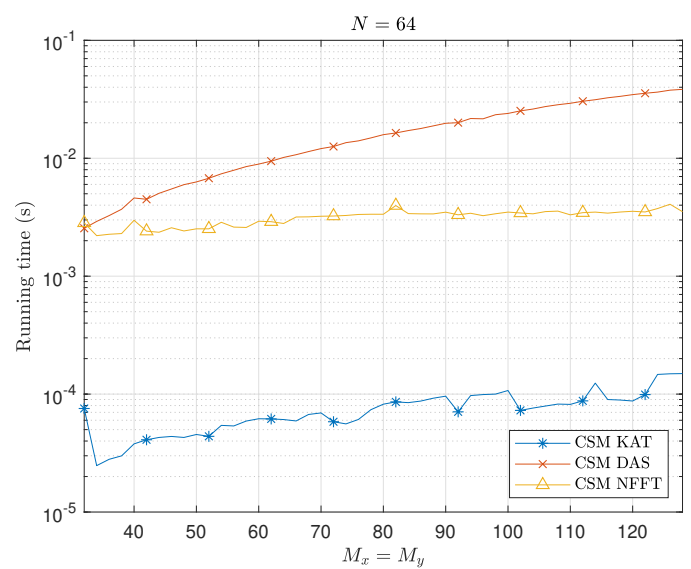

(b)

Figura 10 - Comparação dos tempos de cálculo de uma mesma imagem acústica utilizando CSM-DAS, CSM-KAT e NFFT baseada em CSM. As curvas correspondem a arranjos separáveis com $N=36$ (a) e $N=64$ (b) microfones. Cada ponto corresponde a uma média de 100 realizações de uma distribuição com três fontes na frequência de $10 \mathrm{kHz}$.

\subsubsection{DAMAS acelerado por KAT}

DAMAS, se implementado com convolução, assume a forma da equação (4.48), repetida a seguir:

$$
\mathbf{Y}^{(k+1)}=\max \left\{\mathbf{Y}^{(k)}+\frac{1}{a}\left[\breve{Y}-\left(\mathbf{P} * \mathbf{Y}^{(k)}\right)\right], 0\right\}
$$

em que $\breve{Y}$ é a imagem acústica obtida com DAS.

O cálculo da convolução $\mathbf{B}=\mathbf{P} * \mathbf{Y}^{(k)}$, depois de se fazer $\mathbf{b}=\operatorname{vec}(\mathbf{B})$, pode ser substituído por

$$
\mathbf{b}=\mathbf{A}^{H} \mathbf{A} \operatorname{vec}(\mathbf{Y})=\mathbf{A}^{H} \mathbf{A y}
$$

e o algoritmo vetorizado fica

$$
\mathbf{y}^{(k+1)}=\max \left\{\mathbf{y}^{(k)}+\frac{1}{a}\left[\breve{\mathbf{y}}-\mathbf{A}^{H} \mathbf{A} \mathbf{y}^{(k)}\right], 0\right\} .
$$

De acordo com a seção 5, equação (5.72), o produto $\mathbf{b}=\mathbf{A}^{H} \mathbf{A y}$ pode ser calculado fazendo

$$
\mathbf{B}=\left(\tilde{\mathbf{V}}_{y}^{H} \tilde{\mathbf{V}}_{y}\right) \mathbf{Y}\left(\tilde{\mathbf{V}}_{x}^{T} \tilde{\mathbf{V}}_{x}^{*}\right)
$$

em que as matrizes $\tilde{\mathbf{V}}_{y}^{H} \tilde{\mathbf{V}}_{y}$ e $\tilde{\mathbf{V}}_{x}^{T} \tilde{\mathbf{V}}_{x}^{*}$ devem ser pré-computadas para maior aceleração.

Considerando que a matriz $\mathbf{A}^{H} \mathbf{A}$ é pré-computada, de dimensões $M_{x} M_{y} \times M_{x} M_{y}$, e que y é $M_{x} M_{y} \times 1$, então o cálculo de b requer $M_{x} M_{y} M_{x} M_{y}$ MACs. Supondo também que os produtos $\tilde{\mathbf{V}}_{y}^{H} \tilde{\mathbf{V}}_{y}$, de dimensões $M_{y} \times M_{y}$, e $\tilde{\mathbf{V}}_{x}^{T} \tilde{\mathbf{V}}_{x}^{*}$, de dimensões $M_{x} \times M_{x}$, são précomputados, então o cálculo de $\mathbf{B}$ requer $M_{y} M_{x} M_{x}+M_{y} M_{y} M_{x}$ MACs independentemente da ordem que os produtos forem executados. 


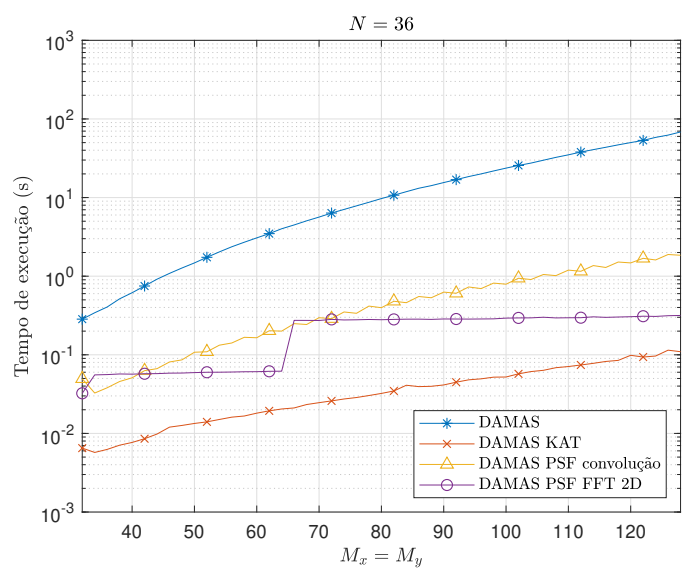

(a)

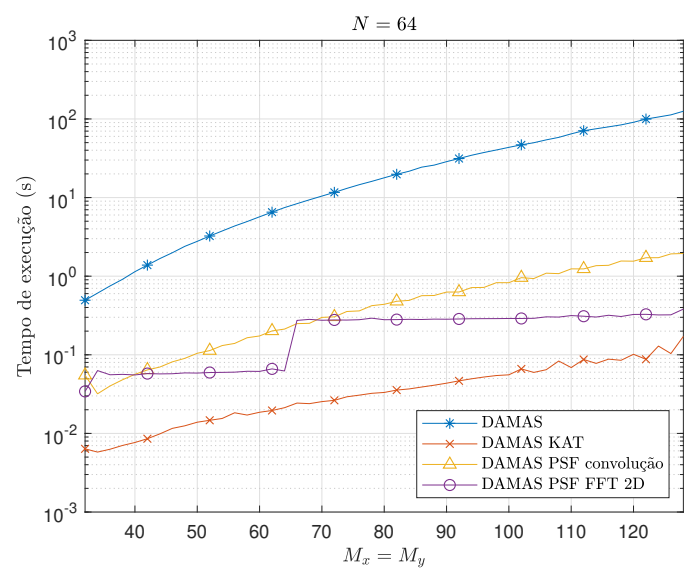

(b)

Figura 11 - Comparação dos tempos de cálculo para quatro implementações diferentes de DAMAS: produto matricial; convolução bidimensional por PSF; convolução bidimensional acelerada por FFT; e acelerada por KAT. As curvas correspondem a arranjos separáveis com $N=36$ (a) e $N=64$ (b) microfones. Cada ponto corresponde a uma média de 100 realizações de uma distribuição com três fontes na frequência de $10 \mathrm{kHz}$ e foram realizadas 100 iterações do algoritmo para cada realização. A FFT bidimensional foi implementada com aplicações sequenciais de FFTs unidimensionais de tamanhos múltiplos de potências de dois.

A aceleração relativa obtida com o uso da KAT é da ordem de

$$
\frac{M_{x} M_{y} M_{x} M_{y}}{M_{y} M_{x} M_{x}+M_{y} M_{y} M_{x}}=\frac{M^{2}}{M \sqrt{M}+M \sqrt{M}}=\frac{M}{2 \sqrt{M}}=\frac{M \sqrt{M}}{2 M}=\frac{\sqrt{M}}{2} .
$$

Note que os algoritmos anteriores tinham acelerações da ordem de $N$ ou $\sqrt{N}$, isto é, dependentes do número de microfones, mesmo o caso mais comum no cálculo de imagens acústicas ser $M \gg N$. No entanto, DAMAS apresenta um ganho de ordem $\sqrt{M}$, de forma que esse algoritmo terá aceleração significativa e ainda mais quando se leva em conta que o cálculo é repetido a cada iteração do algoritmo.

A Figura 11 mostra a aceleração relativa do tempo de cálculo de uma mesma imagem acústica utilizando DAMAS implementado por convolução com a PSF, DAMAS implementado por convolução acelerada por FFT, DAMAS implementado com produto matricial e DAMAS acelerado por KAT.

\subsubsection{Implementações com a matriz A}

Algoritmos que necessitam de uma representação explícita das matrizes $\mathbf{A}, \mathbf{A}^{H}$, $\mathbf{A}^{T}, \mathbf{A}^{*}$, etc, demandam uma grande quantidade de memória, tornando-os mais lentos mesmo para imagens de baixa resolução.

O método DAMAS, por exemplo, utiliza a matriz $\mathbf{A}^{H} \mathbf{A}$ de dimensões $M_{x} M_{y} \times$ $M_{x} M_{y}$. Essa matriz é bem maior que $\mathbf{A}$ e também ocupa muita memória. Nesse caso, a 
implementação de DAMAS por convolução acelerada por FFT pode ser vantajosa. No entanto, a convolução ainda é mais custosa que a KAT. De fato, a KAT pode ser acelerada ainda mais com NFFT ou NNFFT nos casos que as matrizes $\mathbf{V}_{x}, \mathbf{V}_{\mathbf{y}}, \tilde{\mathbf{V}}_{x}, \tilde{\mathbf{V}}_{y}$, etc sejam grandes o suficiente.

A Tabela 1 relaciona a memória ocupada pelas principais matrizes usadas nos algoritmos de estimação de imagens acústicas para $N_{x}=N_{y}=8$. Note que a KAT não só é vantajosa em aceleração computacional, mas também em uso de memória. Por exemplo, para uma imagem $1024 \times 1024$, a matriz A (usada em CSM-DAS) ocupa 68,72 GB enquanto as matrizes $\tilde{\mathbf{V}}_{x}$ e $\tilde{\mathbf{V}}_{y}$ (usadas em CSM-KAT) ocupam 2,1 MB para armazenar a mesma quantidade de informação, isto é, uma redução de 32768 vezes. A diferença gigantesca entre essas duas representações, que armazenam essencialmente a mesma informação, é devida à natureza do produto de Kronecker (Definição 3) que, a partir de duas matrizes $\mathbf{A}$ e $\mathbf{B}$, retorna uma matriz $\mathbf{C}$ de dimensões bem maiores.

Tabela 1 - Memória ocupada pelas principais matrizes complexas usadas no cálculo de imagens acústicas com precisão double.

\begin{tabular}{|c|c|c|c|c|c|}
\hline$M_{x}=M_{y}$ & \multicolumn{5}{|c|}{ Matriz } \\
\hline- & $\mathbf{A}$ & $\tilde{\mathbf{V}}_{x}$ e $\tilde{\mathbf{V}}_{y}$ & $\mathbf{V}$ & $\mathbf{V}_{x}$ e $\mathbf{V}_{\mathbf{y}}$ & $\tilde{\mathbf{V}}_{x}^{T} \tilde{\mathbf{V}}_{x}^{*}$ e $\tilde{\mathbf{V}}_{y}^{H} \tilde{\mathbf{V}}_{y}$ \\
\hline 16 & $1,04 \mathrm{MB}$ & $32,77 \mathrm{kB}$ & $262,14 \mathrm{kB}$ & $4,1 \mathrm{kB}$ & $8,19 \mathrm{kB}$ \\
32 & $67,11 \mathrm{MB}$ & $65,54 \mathrm{kB}$ & $1,04 \mathrm{MB}$ & $8,1 \mathrm{kB}$ & $32,77 \mathrm{kB}$ \\
64 & $268,44 \mathrm{MB}$ & $131,07 \mathrm{kB}$ & $4,19 \mathrm{MB}$ & $16,38 \mathrm{kB}$ & $131,07 \mathrm{kB}$ \\
128 & $1,07 \mathrm{~GB}$ & $262,14 \mathrm{kB}$ & $16,78 \mathrm{MB}$ & $32,77 \mathrm{kB}$ & $524,29 \mathrm{kB}$ \\
256 & $4,29 \mathrm{~GB}$ & $524,29 \mathrm{kB}$ & $67,11 \mathrm{MB}$ & $65,54 \mathrm{kB}$ & $2,1 \mathrm{MB}$ \\
512 & $17,18 \mathrm{~GB}$ & $1,05 \mathrm{MB}$ & $268,44 \mathrm{MB}$ & $131,07 \mathrm{kB}$ & $8,39 \mathrm{MB}$ \\
1024 & $68,72 \mathrm{~GB}$ & $2,1 \mathrm{MB}$ & $1,07 \mathrm{~GB}$ & $262,14 \mathrm{kB}$ & $33,55 \mathrm{MB}$ \\
\hline
\end{tabular}

Na Figura 12, podemos ver a memória requerida para os casos $N=64$ e $N=100$ variando com $M_{x}=M_{y}$. É importante destacar que as duas maiores matrizes, $\mathbf{A}^{H} \mathbf{A}$ e $\mathbf{V}^{H} \mathbf{V}$, dependem apenas de $M$ e não de $N$ de forma que podem crescer muito mesmo para arranjos com poucos microfones. Note também que, apesar do cálculo direto de $\mathbf{A}^{H} \mathbf{A}$ requerer muito mais operações, $\mathbf{V}^{H} \mathbf{V}$ possui o mesmo tamanho na memória. Isso significa que algoritmos a serem discutidos mais adiante que dependem de uma formulação baseada em $\mathbf{V}$, que em geral trabalham com vetores e outras matrizes de tamanhos menores, podem ser tão ineficientes quanto algoritmos envolvendo A e a matriz espectral, pelo menos em termos de memória. 


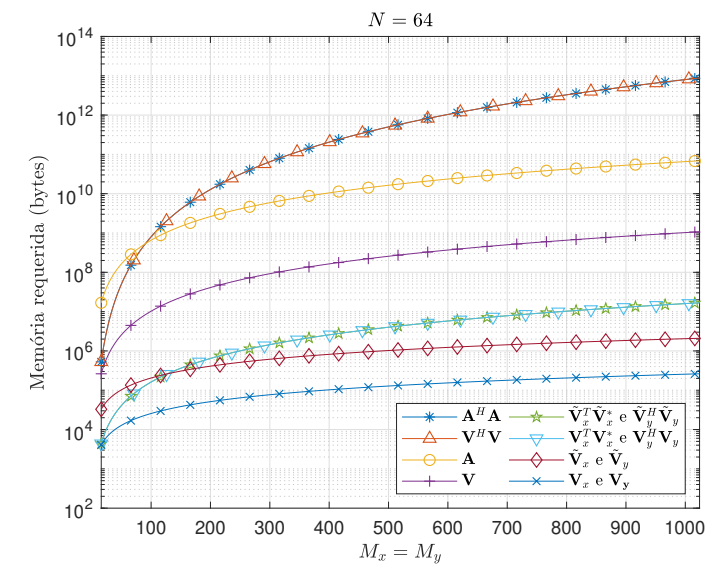

(a)

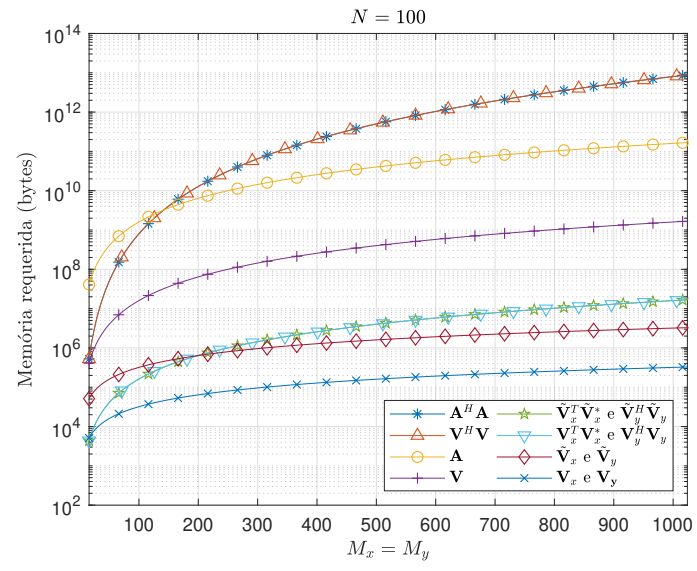

(b)

Figura 12 - Memória ocupada pelas principais matrizes utilizadas nos métodos de estimação de imagens acústicas. O fato de algumas dessas matrizes serem hermitianas foi levado em consideração no cálculo da memória. 



\section{Imageamento por otimização regularizada}

Muitos problemas de reconstrução de imagens podem ser reescritos como problemas de otimização convexa, os quais possuem muitas ferramentas computacionais e algoritmos desenvolvidos especificamente para sua solução. Muitas dessas técnicas, inclusive, foram originalmente desenvolvidas para aplicações de imagens médicas, porém ainda não foram aplicadas a imageamento acústico [4].

Podemos reescrever a equação (4.23) considerando a presença de ruído como

$$
\mathbf{s}=\mathbf{A y}+\operatorname{vec}\left(E\left\{\boldsymbol{\eta} \boldsymbol{\eta}^{H}\right\}\right)=\mathbf{A y}+\sigma^{2} \operatorname{vec}(\mathbf{I})
$$

em que $\boldsymbol{\eta}$ é ruído branco não correlacionado espacialmente. Em geral, a matriz A possui mais colunas (igual ao número de pixels) que linhas (igual ao número de sensores no arranjo ao quadrado), portanto, o sistema da equação (6.1) é indeterminado ${ }^{1}$. Para tornar possível a solução do sistema, modelos relacionados à distribuição de fontes podem ser incorporados como restrições e o problema pode ser resolvido com métodos de otimização.

De forma análoga, podemos trabalhar com a equação (4.12) na forma

$$
\mathrm{x}=\mathrm{Vf}+\boldsymbol{\eta} .
$$

Nos problemas de estimação de imagem acústica que estamos considerando, em geral, o sistema da equação anterior também é indeterminado.

Neste capítulo mostraremos como recuperar os vetores $\mathbf{f}$ e $\mathbf{y}$ das equações (6.1) e (6.2) utilizando métodos de otimização regularizada passíveis de aceleração usando as diversas versões KAT. Os métodos de estimação de imagem acústica que envolvem a formulação (6.2) são denominados métodos diretos, já os que envolvem a formulação (6.1) são chamados métodos de ajuste de covariância.

Como o desempenho computacional é fortemente dependente da arquitetura, a contagem de operações e complexidade do algoritmo serve apenas como uma aproximação. Portanto, neste capítulo apresentamos o ganho esperado dos algoritmos com e sem a aplicação de métodos de aceleração e também mostramos exemplos de tempo de cálculo utilizando apenas um núcleo de um processador ${ }^{2}$ Intel Core i7 de $2 \mathrm{GHz}$.

Apresentamos uma nova extensão do método com regularização por variação total e sua aceleração com a KAT. Estendemos também o uso de algoritmos de homotopia de

1 Isto é, possui mais variáveis que equações.

2 Citamos o processador apenas para referência. Já que o desempenho varia com a arquitetura, um hardware dedicado para operações específicas (como FFT, por exemplo) poderia obter aceleração maior para alguns dos métodos mostrados. No entanto, as relações de números de operações ainda seriam verdadeiras. 
baixa complexidade baseados em coordenadas descendentes para o cálculo de imagens acústicas. Além disso, propomos uma nova classe de algoritmos de homotopia baseados em dicionários decomponíveis em produtos de Kronecker que aproveita a estrutura dos arranjos separáveis e a KAT para conseguir implementações eficientes com baixo uso de memória.

\subsection{Algoritmos esparsos}

Seja $\mathbf{z} \in \mathbb{C}^{M}$ um vetor que representa um sinal esparso (com $K \ll M$ elementos diferentes de zero) que se deseja recuperar. Seja $\mathbf{w} \in \mathbb{C}^{N}$ um vetor conhecido que esteja relacionado com $\mathbf{z}$ pela equação

$$
\mathbf{w}=\Phi \mathbf{z}
$$

em que $\boldsymbol{\Phi}$ é uma matriz conhecida e fixa de dimensões $N \times M$.

Nas aplicações de compressive sensing (CS), o ideal é escolher a matriz $\boldsymbol{\Phi}$ de forma a minimizar ao máximo o número de medidas $N$ tal que $N<M$ e ao mesmo tempo permitir a recuperação de uma grande classe de sinais $\mathbf{z}$ a partir do vetor de medida $\mathbf{w}$ [50]. Na estimação de imagens acústicas, como veremos mais adiante, teremos $\boldsymbol{\Phi}=\mathbf{A}$ ou $\Phi=\mathrm{V}$ de forma que a escolha da matriz $\boldsymbol{\Phi}$ corresponde à escolha da geometria do arranjo. Conforme discutido no seção 5.3, [47] mostrou que os arranjos separáveis de geometria não-redundante (Figura 8) possuem maior potencial de recuperação do sinal z em campo distante. Portanto, neste trabalho consideramos as matrizes que possuem melhor capacidade de reconstrução para aplicações de imagens acústicas.

Como $N<M$, a matriz $\boldsymbol{\Phi}$ será de posto deficiente ou, equivalentemente, o sistema $\mathbf{w}=\mathbf{\Phi} \mathbf{z}$ será subdeterminado. Sabemos que os sistemas subdeterminados possuem infinitas soluções ou nenhuma solução. Uma das maneiras de resolver esse tipo de problema é restringir as soluções possíveis explorando alguma estrutura previamente conhecida de z. No caso de CS, a estrutura escolhida para $\mathbf{z}$ que permite a solução do problema é a esparsidade.

Seja $\mathbf{z} \in \mathbb{C}^{M}$ um sinal com representação numa base $\left\{\boldsymbol{\psi}_{0}, \boldsymbol{\psi}_{1}, \ldots, \boldsymbol{\psi}_{M-1}\right\}, \boldsymbol{\psi}_{m} \in \mathbb{C}^{M}$, dada por $\mathbf{z}=\sum_{m=0}^{M-1} \theta_{m} \boldsymbol{\psi}_{m}$, em que $\left\{\theta_{0}, \theta_{1}, \ldots, \theta_{M-1}\right\}$ são $M$ coeficientes que formam o vetor $\boldsymbol{\theta} \in \mathbb{C}^{M}$. Podemos, então, escrever $\mathbf{z}=\boldsymbol{\Psi} \boldsymbol{\theta}$, em que $\boldsymbol{\Psi}$ é a matriz cujas colunas são os vetores da base, também chamada dicionário. O mesmo vale se utilizarmos um frame ao invés de uma base formando uma matriz $\Psi \in \mathbb{C}^{N \times M}, N<M$, então existirão infinitas decomposições $\boldsymbol{\theta} \in \mathbb{C}^{M}$ tais que $\mathbf{z}=\boldsymbol{\Psi} \boldsymbol{\theta}, \mathbf{z} \in \mathbb{C}^{N}$. Dizemos que um sinal $\mathbf{z}$ é $K$-esparso na base ou frame $\boldsymbol{\Psi}$ se existir um vetor $\boldsymbol{\theta} \operatorname{com} K \ll M$ elementos diferentes de zero tal que $\mathbf{z}=\boldsymbol{\Psi} \boldsymbol{\theta}$. Também dizemos que o conjunto de índices de $\boldsymbol{\theta}$ correspondentes aos elementos diferentes de zero é chamado suporte de $\boldsymbol{\theta}$. Vamos assumir que $\boldsymbol{\Psi}$ é a matriz identidade de forma que $\mathbf{z}=\boldsymbol{\theta}$ é um sinal esparso na base canônica de $\mathbb{C}^{M}$ sobre $\mathbb{C}$. Note que essa última 
hipótese faz com que $\mathbf{w}$ seja um sinal $K$-esparso no frame $\boldsymbol{\Phi}$, já que $\mathbf{z}$ possui apenas $K$ entradas diferentes de zero, e os índices correspondentes ao elementos não nulos em $\mathbf{z}$ são o suporte.

Definimos a norma $\ell_{0}$ de um vetor como sua quantidade de elementos não nulos. Para impor esparsidade no vetor $\mathbf{z}$ e resolver $\mathbf{w}=\mathbf{\Phi} \mathbf{z}$ podemos minimizar a norma $\ell_{0}$ :

$$
\hat{\mathbf{z}}=\underset{\mathbf{z}}{\arg \min }\|\mathbf{z}\|_{0}, \quad \text { sujeito a } \mathbf{w}=\boldsymbol{\Phi} \mathbf{z}
$$

A norma $\ell_{0}$, apesar de ser chamada assim, não é uma norma, portanto o problema é não convexo. Esse problema pode ser resolvido de forma combinatória quando a matriz $\Psi$ possui algumas propriedades que garantem a unicidade da reconstrução [50], porém é computacionalmente intratável até para dimensões pequenas [51].

A solução do sistema subdeterminado de equações $\mathbf{w}=\mathbf{\Phi} \mathbf{z}$ que possui norma $\ell_{1}$ mínima é, em geral, também a solução mais esparsa do mesmo sistema [52]. Portanto, uma alternativa para a norma $\ell_{0}$ é usar a norma $\ell_{1}$, que também promove esparsidade, e resolver

$$
\hat{\mathbf{z}}=\underset{\mathbf{z}}{\arg \min }\|\mathbf{z}\|_{1}, \quad \text { sujeito a } \mathbf{w}=\mathbf{\Phi} \mathbf{z} .
$$

Esse problema é convexo e conhecido como basis pursuit (BP) [50, 51, 53] e pode ser resolvido eficientemente. BP pode ser reformulado para o caso de ruído aditivo nas medidas, cujo modelo é

$$
\mathbf{w}=\Phi \mathbf{z}+\boldsymbol{\eta}
$$

resultando no problema basis pursuit denoising (BPDN):

$$
\hat{\mathbf{z}}=\underset{\mathbf{z}}{\arg \min }\|\mathbf{z}\|_{1}, \quad \text { sujeito a }\|\boldsymbol{\Phi} \mathbf{z}-\mathbf{w}\|_{2} \leq \epsilon ;
$$

sendo um tipo de problema de otimização estudado em detalhe na literatura de CS [5, 4]. Existem muitos algoritmos eficientes para a solução de problemas do tipo basis pursuit (BP) e variações como mostrado em [54].

No caso da equação $(6.7), \epsilon \geq\|\boldsymbol{\eta}\|_{2}$ é um limite escolhido com base na norma $\ell_{2}$ do ruído $\boldsymbol{\eta}$. Em algumas aplicações é razoável assumir uma distribuição para o ruído em questão, ao invés de uma restrição da magnitude da norma, e alguma característica $a$ priori de probabilidade ou complexidade do sinal observado [50]. Técnicas de otimização também podem ser aplicadas nesses casos e uma das mais populares é o chamado Dantzig selector:

$$
\hat{\mathbf{z}}=\underset{\mathbf{z}}{\arg \min }\|\mathbf{z}\|_{1}, \quad \text { sujeito a }\left\|\Phi^{T}(\mathbf{w}-\mathbf{\Phi} \mathbf{z})\right\|_{\infty} \leq \lambda \sqrt{\log M} \sigma^{2}
$$

em que $\|\cdot\|_{\infty}$ denota a norma $\ell_{\infty}, \lambda$ é um parâmetro que controla a probabilidade da reconstrução de $\mathbf{z}, M$ é o número de elementos em $\mathbf{z}$ e $\sigma^{2}$ é a variância de um ruído AWGN IID. 
Outros métodos de solução possíveis são os chamados algoritmos gulosos ${ }^{3}$. Esses métodos selecionam colunas de $\boldsymbol{\Phi}$ de acordo com a correlação delas com o vetor $\mathbf{w}$ calculada através de um produto interno [50].

\section{$6.2 \mathrm{MP}$}

Matching pursuit (MP) [55], um dos algoritmos mais simples de aproximação esparsa, produz iterativamente uma sequência de aproximações esparsas para um sinal. Um novo elemento para ser adicionado ao suporte é selecionado com base no maior produto interno do resíduo da iteração atual $\mathbf{r}_{k}$ e as colunas do dicionário $\boldsymbol{\Phi}$, depois o vetor $\mathbf{z}$ é atualizado e o resíduo da iteração seguinte calculado.

Seja um dicionário $\boldsymbol{\Phi}$, um sinal conhecido $\mathbf{w}$, um vetor $\mathbf{z}$ esparso que se deseja conhecer e considerando ruído aditivo $\boldsymbol{\eta}$ nas medidas conforme (6.6), em que

$$
\Phi=\left[\begin{array}{llll}
\phi_{0} & \phi_{1} & \cdots & \phi_{M-1}
\end{array}\right]
$$

MP recebe como entrada o sinal w, a matriz $\Phi$ e o número de iterações $K$; e retorna como saída o vetor $\mathbf{z}$ que estima o sinal ideal. A inicialização do algoritmo consiste em fazer o resíduo inicial $\mathbf{r}_{0}=\mathbf{w}$ e iniciar o vetor de saída $\mathbf{z}_{0}=\mathbf{0}$.

Seja o conjunto de índices $\Lambda=\{0,1, \ldots, M-1\}$. Na $k$-ésima iteração o algoritmo procura o maior valor no conjunto de projeções $\mathcal{P}_{k-1}=\left\{\left\langle\mathbf{r}_{k-1}, \boldsymbol{\phi}_{m}\right\rangle /\left\|\boldsymbol{\phi}_{m}\right\|_{2}\right\}, m \in \Lambda$. Se as colunas do dicionário possuírem norma $\ell_{2}$ unitária, essa procura é equivalente a calcular

$$
\mathbf{p}_{k}=\boldsymbol{\Phi}^{H} \mathbf{r}_{k-1}
$$

e resolver o problema de otimização

$$
\lambda_{k}=\underset{m=0, \ldots, M-1}{\arg \max }\left|\left[\mathbf{p}_{k}\right]_{m}\right| .
$$

Depois de encontrado o índice $\lambda_{\mathbf{k}}$ do novo elemento do suporte, o algoritmo atualiza o vetor de saída fazendo

$$
\left[\mathbf{y}_{k}\right]_{\lambda_{k}}=\left[\mathbf{y}_{k-1}\right]_{\lambda_{k}}+\left[\mathbf{p}_{k}\right]_{\lambda_{k}}
$$

e atualiza o resíduo subtraindo a projeção ortogonal do resíduo anterior $\mathbf{r}_{k-1}$ no espaço gerado por $\phi_{\lambda_{k}}$, isto é,

$$
\mathbf{r}_{k}=\mathbf{r}_{k-1}-\left[\mathbf{p}_{k}\right]_{\lambda_{k}} \boldsymbol{\phi}_{\lambda_{k}}
$$

O problema de MP é que as colunas do dicionário não são necessariamente vetores mutuamente ortogonais, de forma que subtrair os resíduos pode introduzir componentes no sinal que não são ortogonais ao espaço gerado pelas colunas anteriores. Esse problema 
pode ser contornado com uso de orthogonal matching pursuit (OMP), que trataremos na próxima seção, já que OMP faz a projeção ortogonal de w no espaço gerado em todas as colunas anteriores do dicionário correspondentes ao suporte ao invés de fazer a projeção em apenas uma coluna por iteração [56].

A complexidade do algoritmo MP será dominada pelos cálculos do produto $\boldsymbol{\Phi}^{H} \mathbf{r}_{k-1}$ a cada iteração. Porém, caso seja possível armazenar os produtos internos $\left\langle\boldsymbol{\phi}_{i}, \boldsymbol{\phi}_{i}\right\rangle$, isto é, armazenar a matriz de Gram $\boldsymbol{\Phi}^{H} \boldsymbol{\Phi}$ podemos atualizar as projeções $\mathbf{p}_{k}$ a cada iteração sem calcular o produto do dicionário pelo resíduo fazendo $[56,57]$

$$
\mathbf{p}_{k}=\mathbf{p}_{k}-\left[\mathbf{p}_{k}\right]_{\lambda_{k}}\left[\mathbf{V}^{H} \mathbf{V}\right]_{:, \lambda_{k}}
$$

Para estimar imagens acústicas, resolvemos o problema

$$
\hat{\mathbf{f}}=\underset{\mathbf{f}}{\arg \min }\|\mathbf{f}\|_{0}, \quad \text { sujeito a } \mathbf{x}=\mathbf{V f}
$$

fazendo $\Phi=\mathbf{V}, \mathbf{w}=\mathbf{x}$ e $\mathbf{z}=\mathbf{f}$. Esse método será denominado MP-X.

Os produtos do dicionário pelo resíduo $\mathbf{p}_{k}=\mathbf{V}^{H} \mathbf{r}_{k-1}$ podem ser acelerados pela KAT adjunta:

$$
\mathbf{P}_{k}=\mathbf{V}_{y}^{H} \mathbf{R}_{k-1} \mathbf{V}_{x}^{*}
$$

em que $\mathbf{p}_{k}=\mathbf{V}^{H} \mathbf{r}_{k-1}, \mathbf{p}_{k}=\operatorname{vec}\left\{\mathbf{P}_{k}\right\}$ e $\mathbf{r}_{k-1}=\operatorname{vec}\left\{\mathbf{R}_{k-1}\right\}$, em que $\mathbf{R}_{k-1}$ possui dimensões $N_{x} \times N_{y}$.

No caso do cálculo recursivo do vetor de projeções $\mathbf{p}_{k}$ (equação (6.14)), como não há necessidade de calcular $\mathbf{V}^{H} \mathbf{r}_{k-1}$ a cada iteração, a KAT adjunta só pode ser usada para acelerar os cálculos da inicialização. Esse algoritmo recursivo, apesar de eliminar a operação mais custosa do método de MP, requer muita memória conforme a matriz $\mathbf{V}^{H} \mathbf{V}$, de dimensões $M \times M$, cresce, portanto focamos a análise na implementação que realiza os produtos do dicionário pelo resíduo a cada iteração.

O produto do dicionário pelo resíduo requer $8 M N-2 M$ operações reais por iteração, incluindo multiplicações e adições; a atualização do resíduo requer $8 N$ operações reais por iteração; a atualização do vetor de resposta requer apenas 2 operações reais por iteração; e a procura do maior valor do conjunto de projeções requer $4 N$ operações reais por iteração. Portanto o custo total de MP em $K$ iterações será $O_{\mathrm{MP}}=(8 M N-2 M+12 N+2) K+8 M N-$ $2 M$, em que o último termo corresponde à inicialização do vetor de projeções. A aplicação da KAT, supondo $M_{x}=M_{y}$ e $N_{x}=N_{y}$, reduz o custo total de operações para $O_{\mathrm{MP} \text { KAT }}=$ $(4 N \sqrt{M}-2 \sqrt{N M}+4 M \sqrt{N}-2 M+12 N+2) K+4 N \sqrt{M}-2 \sqrt{N M}+4 M \sqrt{N}-2 M$. Para $M$ grande, esse ganho tende ao da KAT. Na Figura 13, vemos a comparação dos tempos de cálculo para MP-X com e sem a KAT para duas geometrias separáveis, $N=64$ e $N=100$, ao se variar o tamanho da imagem $M$ e a quantidade de iterações $K$. 


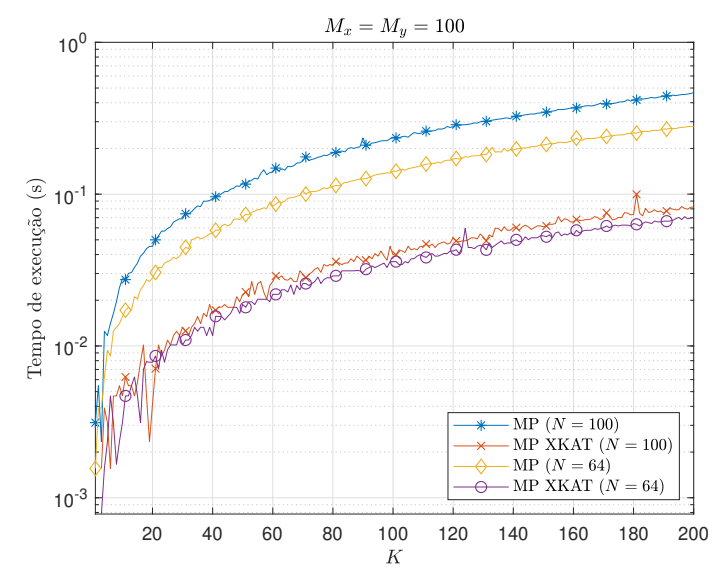

(a)

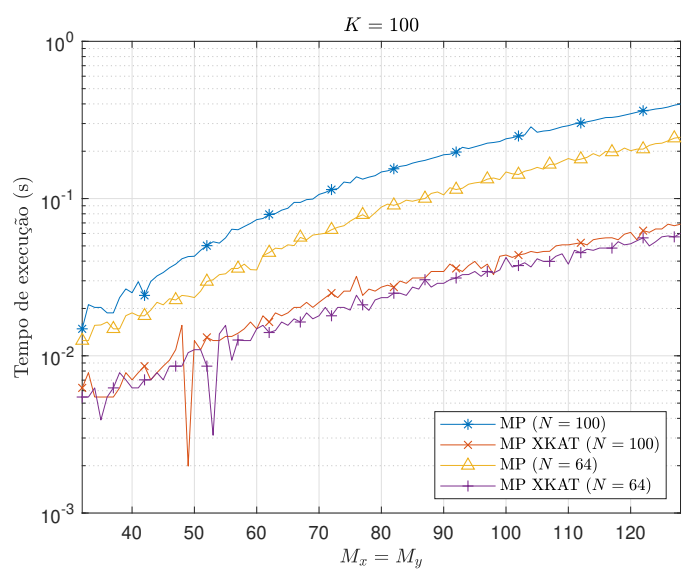

(b)

Figura 13 - Comparação dos tempos de execução para MP-X considerando as acelerações conseguidas com aplicação da KAT. Todos as imagens foram calculadas na frequência de $11 \mathrm{kHz}$ com arranjos de geometria separável para uma distribuição de 10 fontes pontuais e cada ponto corresponde a uma média de 100 realizações. As rotinas foram implementadas em $\mathrm{C} / \mathrm{C}++$ e chamadas no Matlab na forma de funções MEX.

\subsection{OMP}

Orthogonal matching pursuit (OMP) é um algoritmo guloso que pode ser aplicado a problemas de estimação esparsa. Seja um dicionário $\boldsymbol{\Phi}$, um sinal conhecido w e um vetor $\mathbf{z}$ que se deseja conhecer. Considerando ruído aditivo $\boldsymbol{\eta}$ nas medidas, temos

$$
\mathbf{w}=\Phi \mathbf{z}+\boldsymbol{\eta}
$$

em que

$$
\Phi=\left[\begin{array}{llll}
\phi_{0} & \phi_{1} & \cdots & \phi_{M-1}
\end{array}\right]
$$

Seja o conjunto de índices $\Lambda=\{0,1, \ldots, M-1\}$, denotamos $\Lambda_{k} \subset \Lambda$ um conjunto de índices ordenado tal que $\left|\Lambda_{k}\right|=k$. Portanto a matriz $\boldsymbol{\Phi}_{\Lambda_{k}}$ é composta a partir de um subconjunto das colunas de $\Phi$ escolhidas e ordenadas de acordo com $\Lambda_{k}$.

OMP recebe como entrada o sinal w, a matriz $\boldsymbol{\Phi}$ e o nível de esparsidade $K$ do sinal ideal; e retorna como saída o vetor $\mathbf{z}$ que estima o sinal ideal. A inicialização do algoritmo consiste em iniciar o contador $k=1$, iniciar o conjunto de índices $\Lambda_{0}=\varnothing$, iniciar a matriz $\Phi_{\Lambda_{0}}=\varnothing$ e iniciar o resíduo $\mathbf{r}_{0}=\mathbf{w}$.

$\mathrm{Na} k$-ésima iteração, o algoritmo procura o maior valor do conjunto de projeções $\mathcal{P}_{k-1}=\left\{\left\langle\mathbf{r}_{k-1}, \phi_{m}\right\rangle /\left\|\phi_{m}\right\|_{2}\right\}, m \in \Lambda$. Essa procura pode ser feita resolvendo o problema de otimização

$$
\lambda_{k}=\underset{m=0, \ldots, M-1}{\arg \max }\left|\left\langle\mathbf{r}_{k-1}, \boldsymbol{\phi}_{m}\right\rangle /\left\|\boldsymbol{\phi}_{m}\right\|_{2}\right|=\underset{m=1, \ldots, M-1}{\arg \max }\left|\left[\mathbf{r}_{k-1}^{H} \hat{\boldsymbol{\Phi}}\right]_{m}\right|
$$


em que $\hat{\boldsymbol{\Phi}}$ denota a matriz $\boldsymbol{\Phi}$ com colunas normalizadas. Esse passo possui complexidade $\mathcal{O}(M N)$ por iteração.

Depois atualiza-se o conjunto de índices $\Lambda_{k}=\Lambda_{k-1} \cup\left\{\lambda_{t}\right\}$ e a matriz $\Phi_{k}=$ $\left[\begin{array}{ll}\boldsymbol{\Phi}_{k-1} & \boldsymbol{\phi}_{\lambda_{k}}\end{array}\right]$.

Por fim, o algoritmo atualiza o resíduo calculando a projeção ortogonal do sinal de entrada no espaço nulo à esquerda de $\boldsymbol{\Phi}_{\Lambda_{k}}$ denotado $\mathcal{N}\left(\boldsymbol{\Phi}_{\Lambda_{k}}^{H}\right)$ fazendo $\mathbf{r}_{k}=\mathbf{P}_{k}^{\perp} \mathbf{w}$, em que $\mathbf{P}_{k}^{\perp}$ denota a matriz de projeção ortogonal. Temos $\mathbf{P}_{k}^{\perp}=\mathbf{I}-\mathbf{P}_{k}$, em que $\mathbf{P}_{k}$ é a matriz de projeção ortogonal no espaço coluna de $\boldsymbol{\Phi}_{\Lambda_{k}}$ denotado $\mathcal{R}\left(\boldsymbol{\Phi}_{\Lambda_{k}}\right)$. Portanto

$$
\mathbf{r}_{k}=\mathbf{P}_{k}^{\perp} \mathbf{w}=\left(\mathbf{I}-\mathbf{P}_{k}\right) \mathbf{w}=\mathbf{w}-\mathbf{P}_{k} \mathbf{w}
$$

Da definição de matriz pseudoinversa dada por Moore [58], podemos afirmar que a projeção ortogonal do resíduo no espaço $\mathcal{R}\left(\boldsymbol{\Phi}_{\Lambda_{k}}\right)$ é dada por $\mathbf{P}_{k}=\boldsymbol{\Phi}_{\Lambda_{k}} \boldsymbol{\Phi}_{\Lambda_{k}}^{\dagger}$, em que $\boldsymbol{\Phi}_{\Lambda_{k}}^{\dagger}=\left(\boldsymbol{\Phi}_{\Lambda_{k}}^{H} \boldsymbol{\Phi}_{\Lambda_{k}}\right)^{-1} \boldsymbol{\Phi}_{\Lambda_{k}}^{H}$ denota a matriz pseudoinversa de $\boldsymbol{\Phi}_{\Lambda_{k}}$.

A atualização do resíduo é equivalente a resolver o problema de mínimos quadrados

$$
\mathbf{z}_{k}=\underset{\mathbf{z}}{\arg \min }\left\|\mathbf{w}-\boldsymbol{\Phi}_{\Lambda_{k}} \mathbf{z}\right\|_{2}
$$

e calcular o valor para a nova aproximação do resíduo

$$
\mathbf{r}_{k}=\mathbf{w}-\boldsymbol{\Phi}_{\Lambda_{k}} \mathbf{z}_{k}
$$

Incrementa-se $k$ e o algoritmo repete esse processo se $k \leq K$. A estimativa $\hat{\mathbf{z}}$ do sinal ideal na saída do algoritmo possui entradas diferentes de zero somente nos índices de $\Lambda_{m}$ e o valor de $\mathbf{z}$ no componente $\lambda_{j}$ é igual ao $j$-ésimo componente de $\mathbf{z}_{k}$ [59].

A ortogonalização do resíduo tem custo por iteração de no máximo $\mathcal{O}\left(N k+N k^{2}+k^{3}\right)$ caso seja realizado o cálculo direto da pseudoinversa, portanto o custo total da $k$-ésima iteração de OMP é $\mathcal{O}\left(M N+N k+N k^{2}+k^{3}\right)$. A seguir veremos que essas complexidade pode ser reduzida.

Suponha que uma matriz $\mathbf{A}_{k-1} \in \mathbb{C}^{(k-1) \times(k-1)}$ possua decomposição de Cholesky conhecida dada por $\mathbf{A}_{k-1}=\mathbf{L}_{k-1} \mathbf{L}_{k-1}^{H}$, então a decomposição da matriz

$$
\mathbf{A}_{k}=\left[\begin{array}{cc}
\mathbf{A}_{k-1} & \mathbf{h} \\
\mathbf{h}^{H} & c
\end{array}\right] \in \mathbb{C}^{k \times k}
$$

tal que $\mathbf{A}_{k}=\mathbf{L}_{k} \mathbf{L}_{k}^{H}$ será

$$
\mathbf{L}_{k}=\left[\begin{array}{cc}
\mathbf{L}_{k-1} & 0 \\
\mathbf{v}^{H} & \sqrt{c-\mathbf{v}^{H} \mathbf{v}}
\end{array}\right]
$$

em que $\mathbf{L}_{k-1} \mathbf{v}=\mathbf{h}[60]$. 
Podemos calcular recursivamente a decomposição de Cholesky de $\boldsymbol{\Phi}_{\Lambda_{k}}^{H} \boldsymbol{\Phi}_{\Lambda_{k}}$ para reduzir os custos computacionais. Para isso, veja que $\boldsymbol{\Phi}_{\Lambda_{k}}=\left[\begin{array}{ll}\boldsymbol{\Phi}_{\Lambda_{k-1}} & \boldsymbol{\phi}_{\lambda_{k}}\end{array}\right]$, isto é, difere da matriz da iteração anterior $\Phi_{\Lambda_{k-1}}$ por apenas uma coluna que foi adicionada ao suporte, portanto

$$
\boldsymbol{\Phi}_{\Lambda_{k}}^{H} \boldsymbol{\Phi}_{\Lambda_{k}}=\left[\begin{array}{c}
\boldsymbol{\Phi}_{\Lambda_{k-1}}^{H} \\
\boldsymbol{\phi}_{\lambda_{k}}^{H}
\end{array}\right]\left[\begin{array}{ll}
\boldsymbol{\Phi}_{\Lambda_{k-1}} & \phi_{\lambda_{k}}
\end{array}\right]=\left[\begin{array}{cc}
\boldsymbol{\Phi}_{\Lambda_{k-1}}^{H} \boldsymbol{\Phi}_{\Lambda_{k-1}} & \boldsymbol{\Phi}_{\Lambda_{k-1}}^{H} \boldsymbol{\phi}_{\lambda_{k}} \\
\boldsymbol{\phi}_{\lambda_{k}}^{H} \boldsymbol{\Phi}_{\Lambda_{k-1}} & \boldsymbol{\phi}_{\lambda_{k}}^{H} \boldsymbol{\phi}_{\lambda_{k}}
\end{array}\right]=\left[\begin{array}{cc}
\boldsymbol{\Phi}_{\Lambda_{k-1}}^{H} \boldsymbol{\Phi}_{\Lambda_{k-1}} & \mathbf{h} \\
\mathbf{h}^{H} & \left\|\boldsymbol{\phi}_{\lambda_{k}}\right\|_{2}^{2}
\end{array}\right]
$$

Temos $c=\left\|\phi_{\lambda_{k}}\right\|_{2}^{2}$ e $\mathbf{h}=\boldsymbol{\Phi}_{\Lambda_{k-1}}^{H} \boldsymbol{\phi}_{\lambda_{k}}$. Se supusermos que a decomposição de Cholesky de $\boldsymbol{\Phi}_{\Lambda_{k-1}}^{H} \boldsymbol{\Phi}_{\Lambda_{k-1}}=\mathbf{L}_{k-1} \mathbf{L}_{k-1}^{H}$ é conhecida, então $\boldsymbol{\Phi}_{\Lambda_{k}}^{H} \boldsymbol{\Phi}_{\Lambda_{k}}=\mathbf{L}_{k} \mathbf{L}_{k}^{H}$ é tal que

$$
\mathbf{L}_{k}=\left[\begin{array}{cc}
\mathbf{L}_{k-1} & \mathbf{0} \\
\mathbf{v}^{H} & \sqrt{\left\|\phi_{\lambda_{k}}\right\|_{2}^{2}-\|\mathbf{v}\|_{2}^{2}}
\end{array}\right]
$$

em que precisamos resolver o sistema triangular

$$
\mathbf{L}_{k} \mathbf{v}=\Phi_{\Lambda_{k-1}}^{H} \phi_{\lambda_{k}}
$$

para obter $\mathbf{v}$.

Para atualizar o resíduo podemos resolver o sistema formado pelas equações normais do passo de mínimos quadrados (o que é equivalente ao produto do resíduo pela pseudoinversa) dadas por

$$
\boldsymbol{\Phi}_{\Lambda_{k}}^{H} \boldsymbol{\Phi}_{\Lambda_{k}} \mathbf{z}_{k}=\mathbf{L}_{k} \mathbf{L}_{k}^{H} \mathbf{z}_{k}=\boldsymbol{\Phi}_{\Lambda_{k}}^{H} \mathbf{w}
$$

O sistema da equação (6.28) possui complexidade $\mathcal{O}\left(k^{3}\right)$, porém pode ser resolvido para $\mathbf{z}_{k}$ em dois passos em que cada passo consiste na solução de um sistema triangular de complexidade $\mathcal{O}\left(k^{2}\right)$ :

$$
\begin{aligned}
\mathbf{L}_{k} \mathbf{g} & =\boldsymbol{\Phi}_{\Lambda_{k}}^{H} \mathbf{w} \\
\mathbf{L}_{k}^{H} \mathbf{z}_{k} & =\mathbf{g} .
\end{aligned}
$$

Se assumirmos que o cálculo do maior valor do conjunto $\mathcal{P}_{k-1}$ continua sendo realizado diretamente, então a complexidade total da implementação com decomposição Cholesky recursiva é $\mathcal{O}\left(M N+N k+k^{2}\right)$. Existem maneiras mais eficientes computacionalmente de implementar o cálculo de $\mathcal{P}_{k-1}$, porém requerem o cálculo prévio de matrizes do tipo $\boldsymbol{\Phi}^{H} \boldsymbol{\Phi}$ resultando num alto uso de memória. Mostraremos que a KAT pode ser aplicada a esse cálculo resultando numa redução significativa do número de operações.

Podemos também adotar uma decomposição QR recursiva no passo de ortogonalização do resíduo. Para isso, mantemos a decomposição QR de $\boldsymbol{\Phi}_{\Lambda_{k-1}}=\mathbf{Q}_{k-1} \mathbf{R}_{k-1}$. Depois de encontrar o índice $\lambda_{k}$ resolvendo o problema de minimização no conjunto de projeções 
$\mathcal{P}_{k-1}$, definimos $\mathbf{v}=\mathrm{Q}_{k-1}^{H} \phi_{\lambda_{k}}$, de forma que a decomposição QR pode ser atualizada fazendo

$$
\begin{aligned}
& \mathbf{R}_{k}=\left[\begin{array}{cc}
\mathbf{R}_{k-1} & \mathbf{v} \\
\mathbf{0}^{T} & \sqrt{\left\|\boldsymbol{\phi}_{\lambda_{k}}\right\|_{2}^{2}-\|\mathbf{v}\|_{2}^{2}}
\end{array}\right] \\
& \mathbf{Q}_{k}=\left[\begin{array}{ll}
\mathbf{Q}_{k-1} & \frac{\boldsymbol{\phi}_{\lambda_{k}}-\mathbf{Q}_{k-1} \mathbf{v}}{\sqrt{\left\|\boldsymbol{\phi}_{\lambda_{k}}\right\|_{2}^{2}-\|\mathbf{v}\|_{2}^{2}}}
\end{array}\right] .
\end{aligned}
$$

A atualização das matrizes possui custo $\mathcal{O}(M+N k)$.

O algoritmo pode calcular o resíduo fazendo $\mathbf{r}_{k}=\mathbf{r}_{k-1}-\mathbf{q}_{k} \mathbf{q}_{k}^{H} \mathbf{w}$ ao custo de $\mathcal{O}\left(N^{2}\right)$ ou atualizar o conjunto de projeções $\mathcal{P}_{k}$ ao custo de $\mathcal{O}(M N)$ sem atualizar o resíduo diretamente.

Por fim, basta resolver dois sistemas triangulares na última iteração $K$ com custo $\mathcal{O}\left(K^{2}\right)$ cada:

$$
\begin{aligned}
& \mathbf{R}_{k}^{H} \mathbf{g}=\boldsymbol{\Phi}_{\Lambda_{k}}^{H} \mathbf{w} \\
& \mathbf{R}_{k} \mathbf{z}_{k}=\mathbf{g} .
\end{aligned}
$$

O algoritmo de OMP pode ser usado para resolver o problema

$$
\hat{\mathbf{f}}=\underset{\mathbf{f}}{\arg \min }\|\mathbf{f}\|_{0}, \quad \text { sujeito a } \mathbf{x}=\mathbf{V f} .
$$

fazendo $\boldsymbol{\Phi}=\mathbf{V}, \mathbf{w}=\mathbf{x}$ e $\mathbf{z}=\mathbf{f}$. Esse método será denominado OMP-X para o cálculo de imagens acústicas.

O passo de ortogonalização do resíduo pode ser calculado de forma eficiente usando as implementações recursivas com decomposições QR ou Cholesky. Além disso, podemos explorar a estrutura da matriz $\mathbf{V}$ quando assumimos as mesmas hipóteses de geometria de arranjo e amostragem que permitem aplicação da KAT.

Assumindo que as colunas de $\mathbf{V}$ já foram normalizadas previamente, temos

$$
\lambda_{k}=\underset{m=1, \ldots, M-1}{\arg \max }\left|\left[\mathbf{r}_{k-1}^{H} \mathbf{V}\right]_{m}\right|,
$$

em que $\mathbf{r}_{k-1}^{H} \mathbf{V}=\left(\mathbf{V}^{H} \mathbf{r}_{k-1}\right)^{H}$. Sejam $\mathbf{p}=\mathbf{V}^{H} \mathbf{r}_{k-1}, \mathbf{p}=\operatorname{vec}\{\mathbf{P}\}$ e $\mathbf{r}_{k-1}=\operatorname{vec}\left\{\mathbf{R}_{k-1}\right\}$, em que $\mathbf{R}_{k-1}$ possui dimensões $N_{x} \times N_{y}$. Portanto podemos aplicar a KAT adjunta para acelerar esse produto matriz vetor fazendo

$$
\mathbf{P}=\mathbf{V}_{y}^{H} \mathbf{R}_{k-1} \mathbf{V}_{x}^{*}
$$

A Figura 14 ilustra a comparação dos tempos de execução para uma mesma imagem acústica considerando a aplicação da KAT e dos métodos rápidos baseados nas 


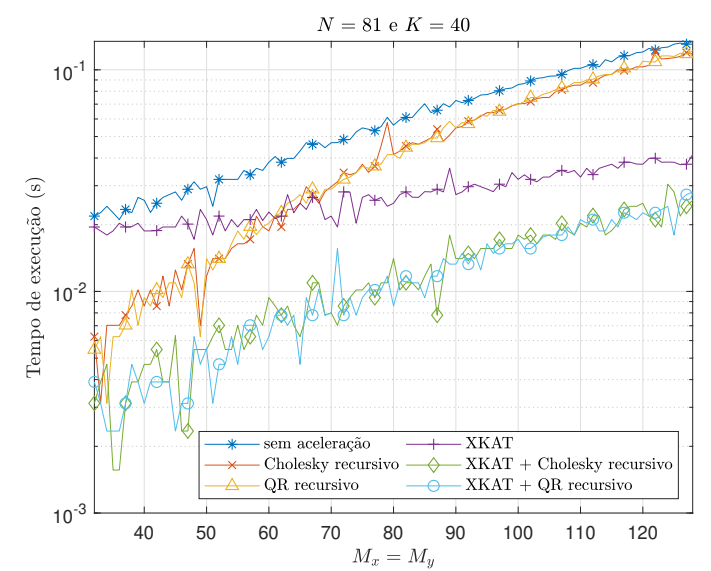

(a)

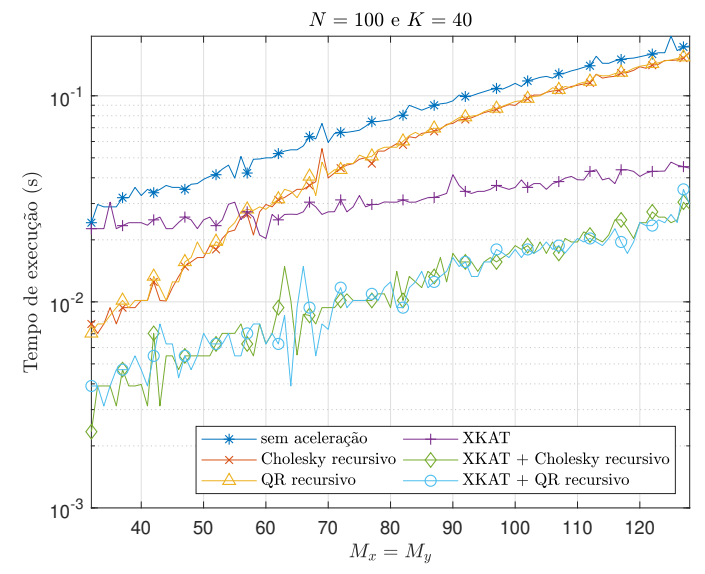

(c)

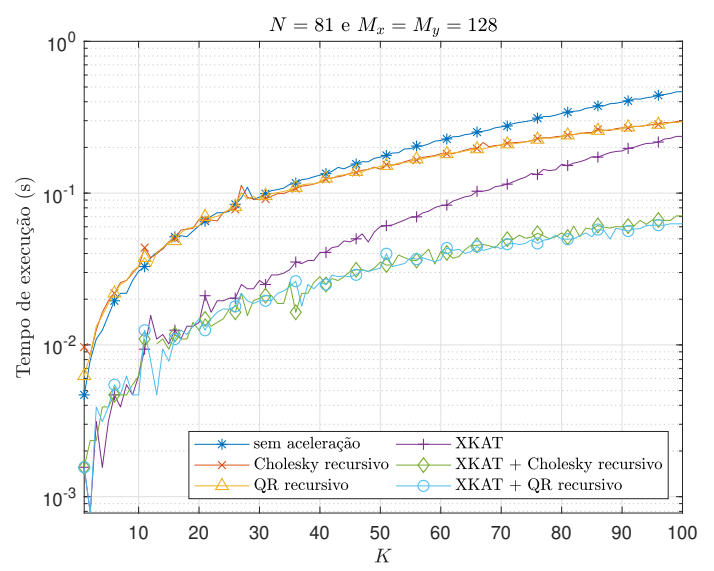

(b)

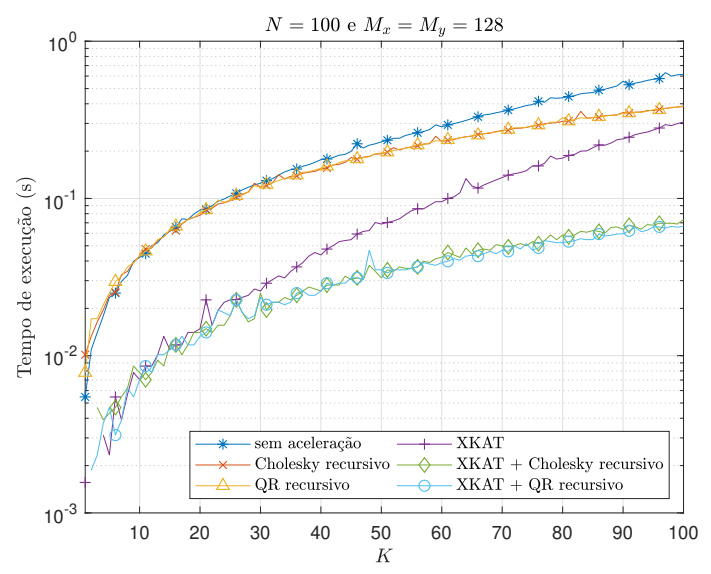

(d)

Figura 14 - Comparação dos tempos de execução para OMP-X considerando as acelerações conseguidas com aplicação da KAT e de implementações recursivas baseadas nas decomposições de Cholesky e QR. Todos as imagens foram calculadas na frequência de $11 \mathrm{kHz}$ com arranjos de geometria separável e cada ponto corresponde a uma média de 100 realizações. As rotinas foram implementadas em $\mathrm{C} / \mathrm{C}++$ e chamadas no Matlab na forma de funções MEX.

decomposições QR e de Cholesky. Nas Figuras 14a e 14b, que correspondem a um arranjo de $N=81$ microfones, notamos que, para uma esparsidade fixa $(K=40)$ e o tamanho da imagem $M=M_{x} M_{y}$ variando, a aceleração conseguida pela KAT sozinha domina quando $M$ aumenta e o ganho adicional conseguido ao aplicar as decomposições em conjunto com a KAT é pequeno, porém para $M$ pequeno o ganho adicional é significativo. Nas curvas da Figura 14b, o tamanho da imagem é mantido fixo e esparsidade varia resultando em um domínio do passo de ortogonalização do resíduo. Isso nos diz que no caso típico de uma imagem acústica esparsa com $M \gg K$, a KAT é o fator mais importante na diminuição da complexidade computacional do algoritmo, porém a combinação de KAT com uma implementação rápida do OMP sempre é vantajosa em todos os cenários. Nas Figuras 14c e 14 d, o número de microfones do arranjo muda para $N=100$, mostrando que as curvas mantêm o mesmo formato porém com tempos de cálculo ligeiramente maiores para todos 
os casos.

No caso da Figura 14, foi dada prioridade para implementações que não armazenam a matriz $\mathbf{V}^{H} \mathbf{V}$, o que iria requerer muita memória. Dependendo dos valores de $N, M$ e $K$ pode ser que possamos trocar um pouco mais de redução no número de cálculos por uma quantidade significativa de memória extra ao armazenar $\mathbf{V}^{H} \mathbf{V}$. Se o dicionário $\mathbf{V}$ for muito grande para ser salvo na memória, a KAT permite uma redução de várias ordens de magnitude na memória requerida salvando apenas $\mathbf{V}_{x}$ e $\mathbf{V}_{y}$ desde que geremos as colunas de $\mathbf{V}_{\Lambda_{k}}$ durante a execução do algoritmo. Além disso, manter as decomposições QR e Cholesky requer memória adicional.

A geração de colunas de $\mathbf{V}_{\Lambda_{k}}$ mencionada anteriormente pode ser feita mapeando os elementos do produto de Kronecker:

$$
[\mathbf{V}]_{i, j}=\left[\mathbf{V}_{x} \otimes \mathbf{V}_{y}\right]_{i, j}=\left[\mathbf{V}_{x}\right]_{\left\lfloor(i-1) / N_{x}\right\rfloor+1,\left\lfloor(j-1) / M_{x}\right\rfloor+1}\left[\mathbf{V}_{y}\right]_{(i-1) \bmod \left(N_{y}\right)+1,(j-1) \bmod \left(M_{y}\right)+1} .
$$

Também podemos gerar elementos de $\mathbf{V}^{H} \mathbf{V}$ explorando a relação

$$
\mathbf{V}^{H} \mathbf{V}=\left(\mathbf{V}_{x}^{H} \otimes \mathbf{V}_{y}^{H}\right)\left(\mathbf{V}_{x} \otimes \mathbf{V}_{y}\right)=\left(\mathbf{V}_{x}^{H} \mathbf{V}_{x}\right) \otimes\left(\mathbf{V}_{y}^{H} \mathbf{V}_{y}\right)
$$

e depois mapear os elementos do produto de Kronecker.

\subsection{Compressive beamforming}

Os autores de [51] propõem usar CS para estimação de direção de chegada (DOA) com arranjos de sensores. O método mais simples para estimação de DOA é beamforming DAS, já explorado anteriormente neste trabalho. DAS, além de estimar as direções de chegada das fontes, também fornece estimativas das respectivas intensidades. No entanto, existem algoritmos que podem ser usados só para estimativa de direção de chegada como MUSIC [61] e ESPRIT [62, 63].

Em [51], compressive beamforming (CB) é comparado a DAS, MVDR e MUSIC para um arranjo linear de $N$ sensores, assumindo campo distante, processamento de banda estreita e um problema confinado a duas dimensões. O modelo de sinal é similar ao definido nesse trabalho, a diferença é que [51] se preocupa apenas em estimar o ângulo $\theta \in\left[-90^{\circ}, 90^{\circ}\right]$ em relação ao eixo do arranjo, já que utiliza um arranjo linear. Temos

$$
\mathbf{x}=\mathbf{V f}+\boldsymbol{\eta}
$$

em que as colunas de $\mathbf{V}$ são os vetores diretores correspondentes ao ângulos $\theta_{m}$ escolhidos numa grade fina, $\mathbf{f}$ é o vetor desconhecido das $M$ fontes, $\mathbf{x}$ é o vetor medido pelos sensores do arranjo e $\boldsymbol{\eta}$ é um ruído aditivo gaussiano. 
Vamos assumir poucas fontes, isto é, f possui $K \ll M$ elementos diferentes de zero. [51] propõe resolver o problema de BPDN dado por

$$
\min _{\mathbf{f}}\|\mathbf{f}\|_{1}, \quad \text { sujeito a }\|\mathbf{V f}-\mathbf{x}\|_{2} \leq \epsilon
$$

em que $\epsilon \geq\|\boldsymbol{\eta}\|_{2}$ e $\mathbf{x}$ é obtido de um único snapshot, ou seja, similar a como os algoritmos X-KAT e X-DAS obtêm x. Os autores de [51] propõem também uma versão do problema para vários snapshots. $\mathrm{CB}$ apresenta resolução mais alta que os outros métodos mesmo nos casos de poucos snapshots, fontes correlacionadas e maiores níveis de ruído.

Esse método pode ser estendido para a estimação de imagens acústicas e para uma grade retangular amostrada de alguma forma específica no espaço $U$. Veremos que, no caso de geometria e amostragem separáveis, podemos aplicar a KAT para acelerar o cálculo de imagens por CB.

Os autores de [51] utilizam o pacote CVX [64, 65] para problemas de otimização convexa, porém preferimos utilizar o pacote SPGL1 [66, 5], específico para os problemas esparsos BP, BPDN e Lasso, conforme sugerido por $[17,18]$. A vantagem do pacote SPGL1 sobre o pacote CVX para essa aplicação consiste no fato de SPGL1 requerer apenas produtos matriz-vetor do tipo $\mathbf{V}^{H} \mathbf{x}$ e $\mathbf{V x}$, em que $\mathbf{x}$ não necessariamente representa o vetor medido nos microfones, que podem ser facilmente acelerados pelas KATs adjunta e direta.

SPGL1 possui duas tarefas que correspondem à maior parte do tempo de execução do algoritmo: um produto por iteração matriz-vetor da forma $\mathbf{V}^{H} \mathbf{x}$ e um produto por iteração da forma $\mathbf{V} \mathbf{x}$, totalizando um custo de $\mathcal{O}(N M)$; e uma operação de projeção por iteração que possui custo $\mathcal{O}(M \log M)$ [5]. Os dois produtos podem ser acelerados pela KAT causando uma redução significativa do tempo de execução. Note que isso não significa que o algoritmo realize apenas os dois produtos matriz-vetor a cada iteração, já que mais produtos matriz-vetor são requeridos na inicialização e em alguns passos condicionais na execução do algoritmo, porém é correto afirmar que esses dois produtos por iteração correspondem à grande maioria das operações desse tipo.

Na Figura 15, podemos ver a fração do tempo total de cálculo de uma imagem acústica pelo método de compressive beamforming que é gasta pelos produtos matriz-vetor, pela projeção e pelas outras tarefas realizadas pelo algoritmo. Nas Figuras 15a e 15b observamos que os produtos matriz-vetor correspondem a aproximadamente $65 \%$ do tempo total de execução do algoritmo, para uma geometria com $N=100$ e uma imagem de tamanho $M_{x}=M_{y}=100$, sendo a operação mais custosa. Após a aplicação da KAT ao algoritmo, a porcentagem do tempo de cálculo de produtos matriz-vetor cai para cerca de $10 \%$ do tempo total. Nas Figuras 15c e 15d observamos o mesmo teste, porém com $N=64$ para que possamos analisar o efeito da variação de $N$; note que as curvas mantêm o mesmo formato com a porcentagem devida à KAT aproximadamente constante conforme 


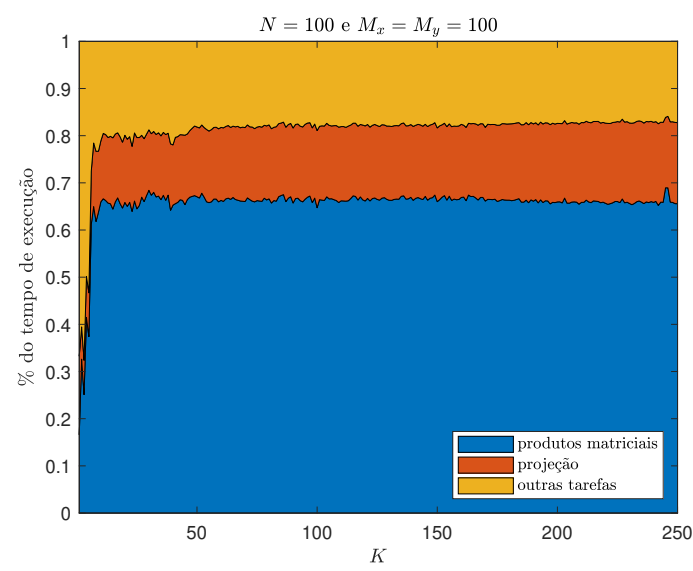

(a)

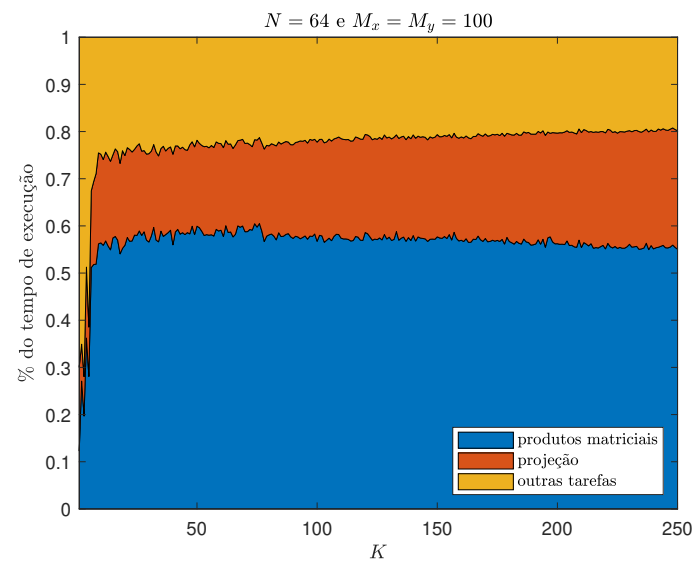

(c)

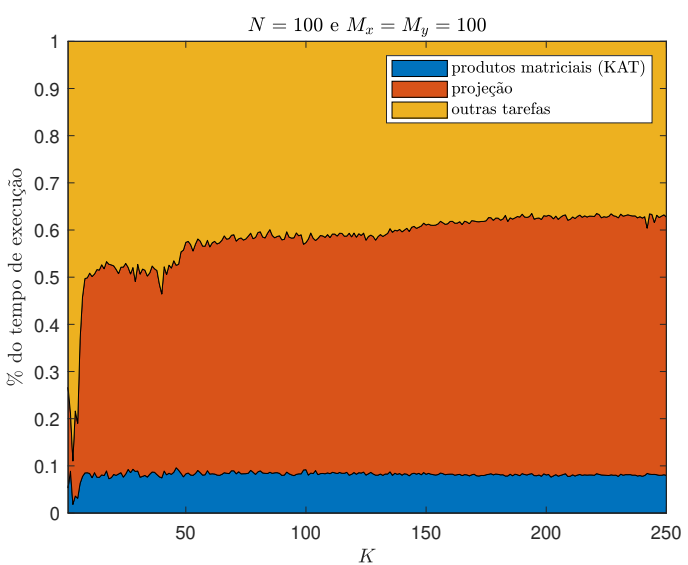

(b)

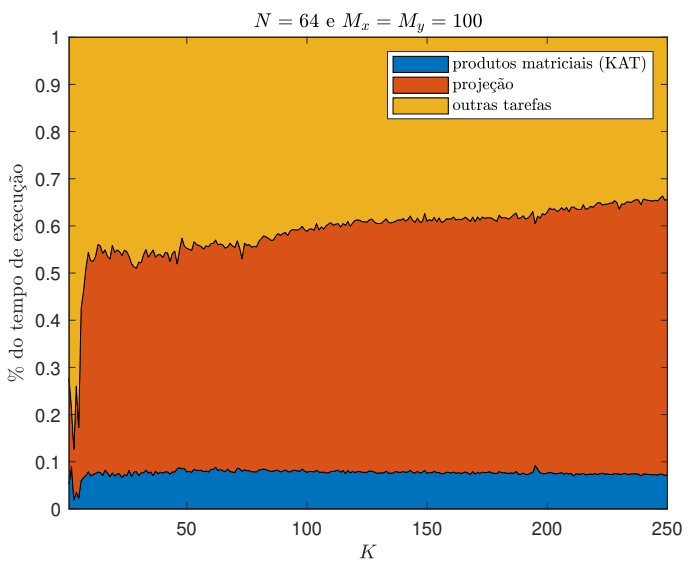

(d)

Figura 15 - Porcentagem do tempo de total de cálculo devida a cada uma das tarefas principais realizadas pelo método de compressive beamforming para estimar uma imagem acústica em função da quantidade de iterações $K$. São mostrados dois casos com arranjos de geometria separável com $N=100$ e $N=64$. Todas as imagens possuíam uma distribuição com 30 fontes pontuais na frequência de $11 \mathrm{kHz}$ e $M_{x}=M_{y}=100$. A quantidade de iterações foi escolhida de forma que o algoritmo ainda não atingiu a convergência, executando todas as operações mencionadas a cada iteração.

o número de iterações $K$ varia, porém agora correspondendo a aproximadamente $55 \%$ do tempo total de execução.

Analisemos agora o perfil de tempo de execução do algoritmo com a variação do tamanho da imagem $M$ e com a quantidade de iterações $K$ fixada, conforme mostrado na Figura 16. Nas Figuras 16a e 16c vemos que a fração do tempo total devida aos produtos matriz-vetor aumenta conforme $M$ aumenta. Nas Figuras 16b e 16d observamos novamente a redução significativa na fração do tempo de execução dos produtos matriz-vetor para cerca de $10 \%$ do tempo total.

Por fim, conforme Figura 17, comparamos tempos de execução do algoritmo com e sem as acelerações proporcionadas pelas KATs direta e adjunta. O ganho esperado por 


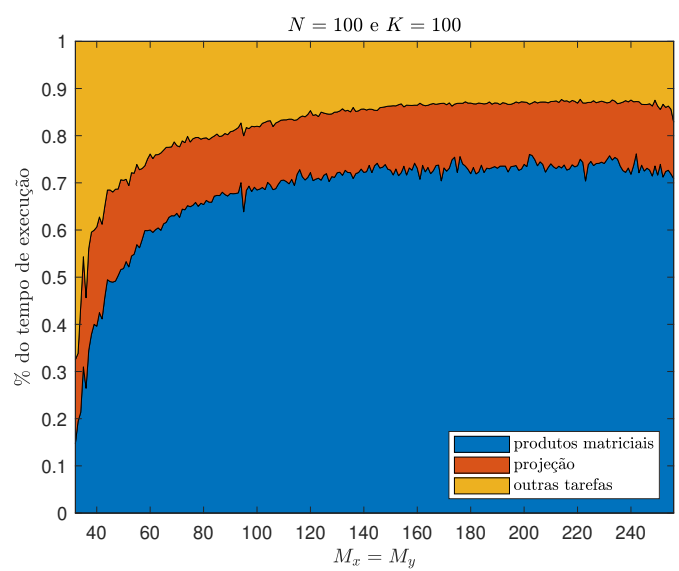

(a)

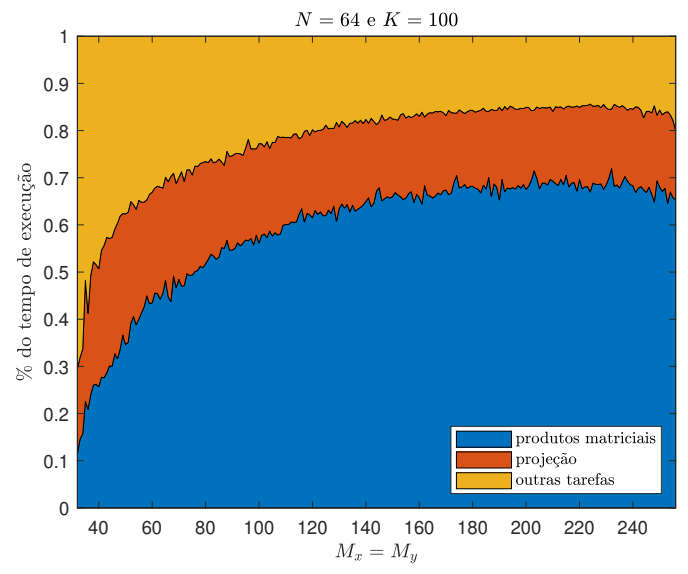

(c)

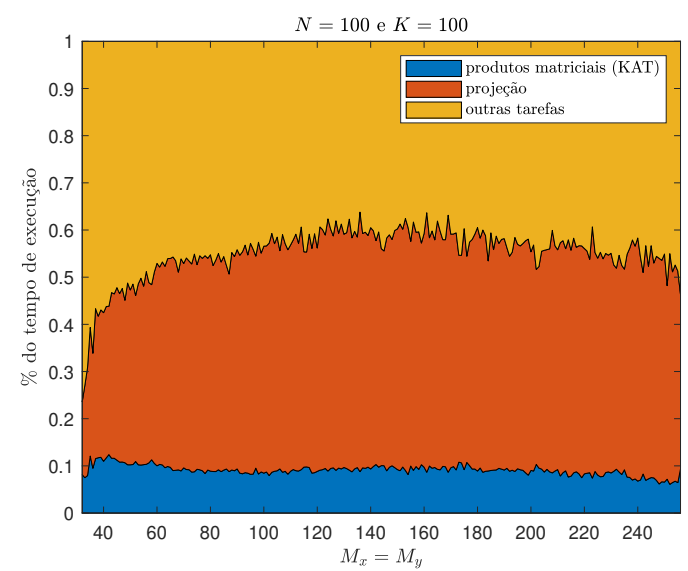

(b)

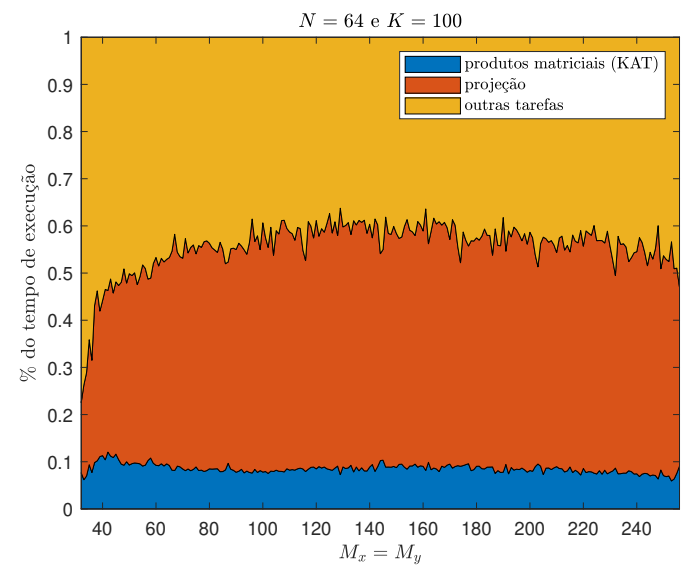

(d)

Figura 16 - Porcentagem do tempo de total de cálculo devida a cada uma das tarefas principais realizadas pelo método de compressive beamforming para estimar uma imagem acústica em função do tamanho da imagem $M_{x}=M_{y}$. São mostrados dois casos com arranjos de geometria separável com $N=100 \mathrm{e}$ $N=64$. Todas as imagens possuíam uma distribuição com 30 fontes pontuais na frequência de $11 \mathrm{kHz}$ e $K=100$. A quantidade de iterações foi escolhida de forma que o algoritmo ainda não atingiu a convergência, executando todas as operações mencionadas a cada iteração.

iteração é uma redução de $\mathcal{O}(M N+M \log M) \operatorname{para} \mathcal{O}(N \sqrt{M}+M \sqrt{N}+M \log M)$.

\subsection{Ajuste de covariância com regularização $\ell_{1}$}

Assumindo uma distribuição de fontes esparsa, podemos aplicar uma restrição para sistemas esparsos a fim de regularizar a solução de (6.1), como foi feito por [67] que propôs resolver o seguinte problema de otimização convexa

$$
\min _{\mathbf{y}, \sigma^{2}}\left\|\mathbf{s}-\mathbf{A y}-\sigma^{2} \operatorname{vec}(\mathbf{I})\right\|_{2}^{2}, \quad \text { sujeito a } y_{i, j} \geq 0, \sigma^{2} \geq 0, \text { e }\|\mathbf{y}\|_{1} \leq \lambda,
$$




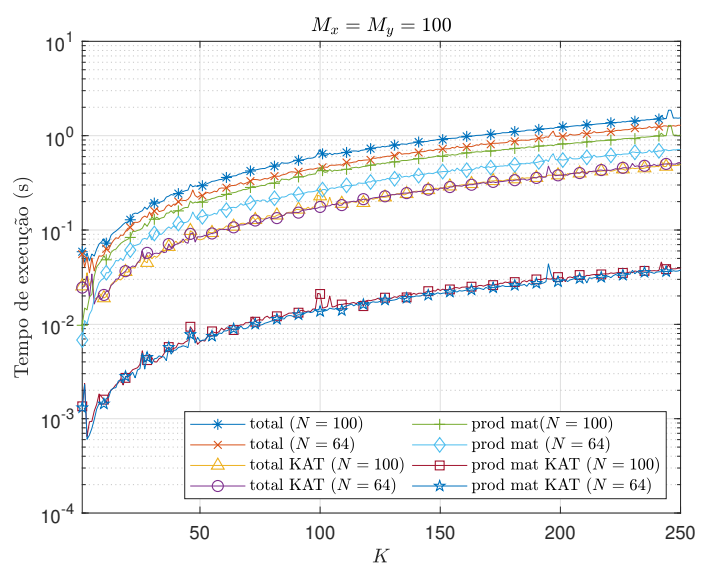

(a)

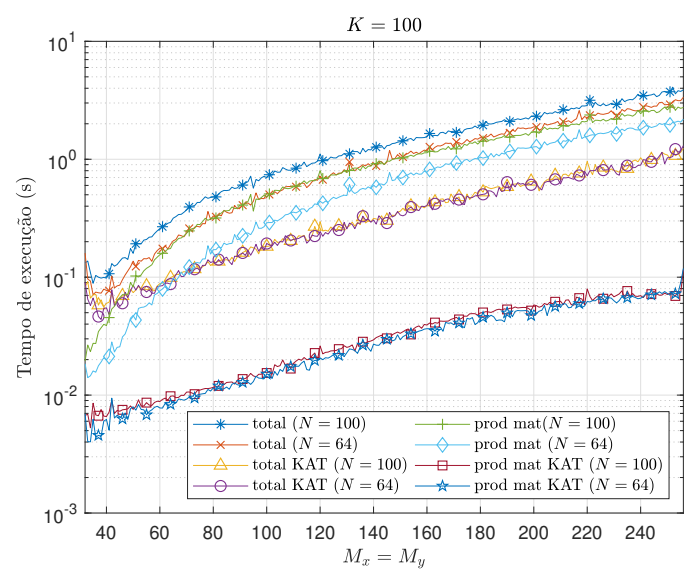

(b)

Figura 17 - Comparação dos tempos de execução para compressive beamforming considerando as acelerações conseguidas com a aplicação das KATs direta e adjunta. Todas as imagens possuíam uma distribuição com 30 fontes pontuais na frequência de $11 \mathrm{kHz}$ e correspondem exatamente aos casos analisados nas Figuras 15 e 16. A opera de projeção foi implementada em C e chamada pelo Matlab [5]. total se refere ao tempo total de cálculo e prod mat ao tempo gasto com os produtos matriz-vetor.

em que $\|\mathbf{y}\|_{1}$ denota a norma $\ell_{1}$ do vetor $\mathbf{y},\|\cdot\|_{2}$ denota a norma $\ell_{2}, M$ é o número total de elementos de y e $\lambda$ é um parâmetro de regularização. A restrição da norma $\ell_{1}$ dada por $\|\mathbf{y}\|_{1} \leq \lambda$ serve para regularizar o problema enquanto força uma solução esparsa.

Devido à natureza do sistema considerado, este método não se aplica caso a distribuição de fontes não seja esparsa. Este método supera DAMAS, pois utiliza regularização quando DAMAS apenas força a não negatividade e também não requer uma convolução intermediária. Mesmo assim, o custo computacional e de memória permanece alto pois é necessário trabalhar com uma representação explícita de $\mathbf{A}$ [8].

Os autores de [17] propõem reescrever o problema (6.42) como um problema BPDN:

$$
\min _{\mathbf{y}}\|\mathbf{y}\|_{1}, \quad \text { sujeito a }\|\mathbf{s}-\mathbf{A y}\|_{2} \leq \epsilon
$$

Note que esse problema é similar ao método de compressive beamforming, porém aqui é usada a matriz espectral em sua forma vetorizada $\mathbf{s}$ ao invés do vetor de medidas nos microfones $\mathbf{x}$.

Novamente utilizamos SPGL1 para resolver um problema de BPDN, fazendo com que a solução só dependa de multiplicações matriz-vetor envolvendo as matrizes $\mathbf{A}$ e $\mathbf{A}^{H}$ que podem ser prontamente aceleradas pela CSM KAT direta e pela CSM KAT adjunta, respectivamente. Com o uso da KAT eliminamos a necessidade de trabalhar com uma representação explícita de $\mathbf{A}$, reduzindo em várias ordens de magnitude o custo de memória e acelerando significativamente os tempos de cálculo. 


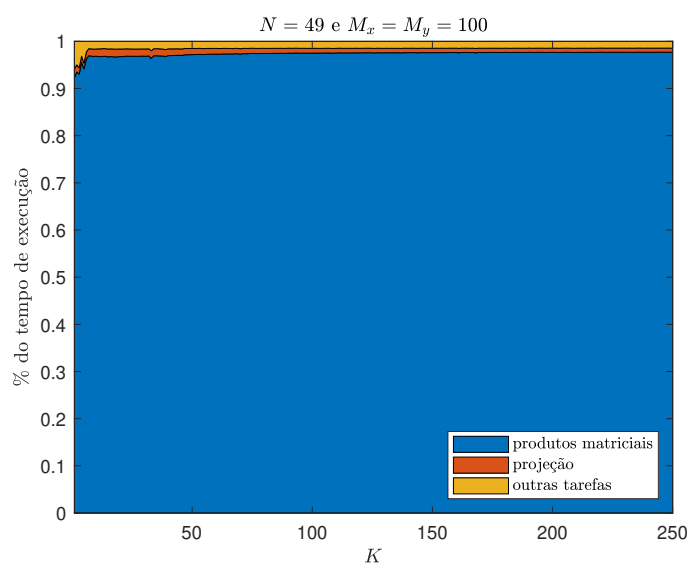

(a)

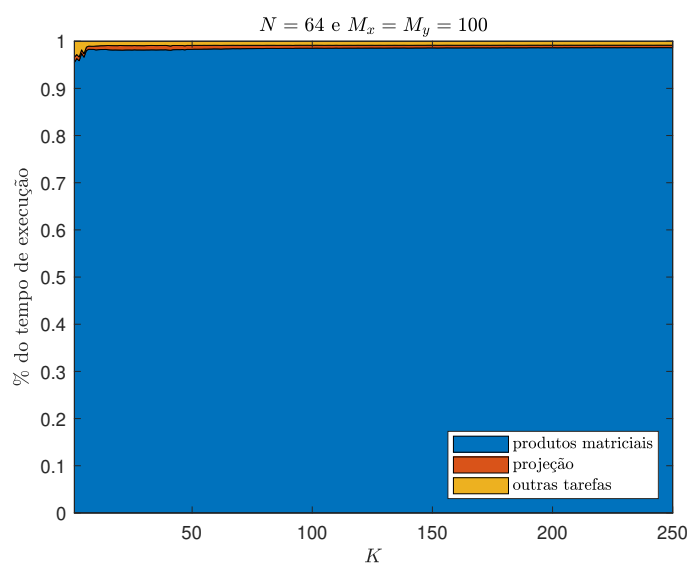

(c)

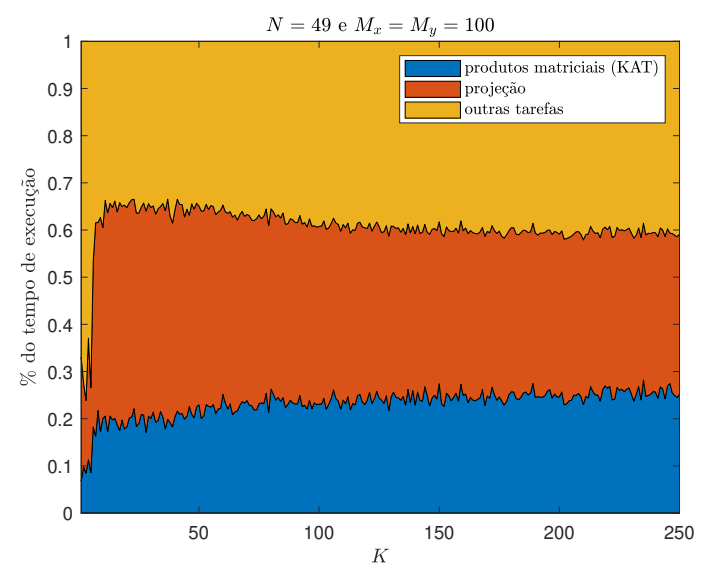

(b)

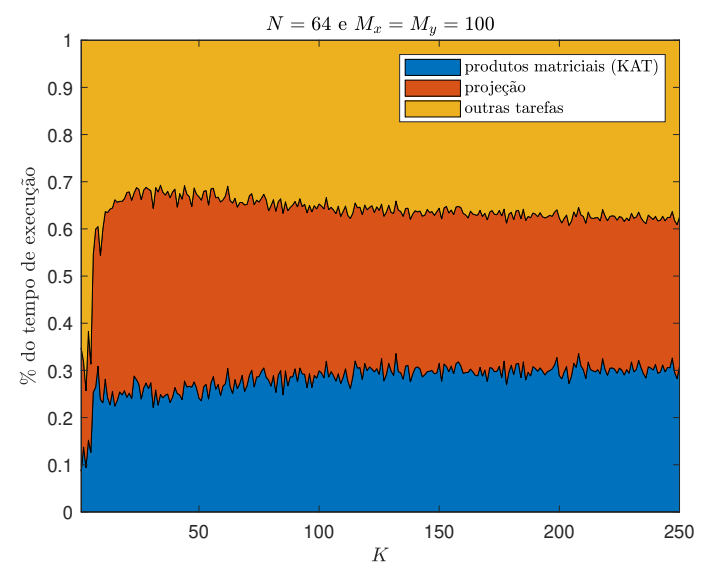

(d)

Figura 18 - Porcentagem do tempo de total de cálculo devida a cada uma das tarefas principais realizadas pelo método de ajuste de covariância reescrito como um problema do tipo BPDN para estimar uma imagem acústica em função da quantidade de iterações $K$. São mostrados dois casos com arranjos de geometria separável com $N=100$ e $N=64$. Todas as imagens possuíam uma distribuição com 30 fontes pontuais na frequência de $11 \mathrm{kHz}$ e $M_{x}=M_{y}=100$. A quantidade de iterações foi escolhida de forma que o algoritmo ainda não atingiu a convergência, executando todas as operações mencionadas a cada iteração.

A Figura 18 ilustra a fração do tempo total de cálculo devido às operações de projeção, produtos matriz vetor e todas as outras tarefas do algoritmo SPGL1 em função da quantidade de iterações para uma imagem fixada. É interessante comparar esse perfil com o da Figura 15 que representa o cálculo de uma imagem de mesmo tamanho $M_{x}=M_{y}=100$. Note que aqui os produtos matriz-vetor são responsáveis por mais de $95 \%$ do tempo total de execução, em contraste com cerca de $65 \%$ do apresentado no método de compressive beamforming da Figura 15. A dominância maior dos produtos matriz-vetor nesse caso se deve ao fato da matriz $\mathbf{A}$ ser muito maior que a matriz $\mathbf{V}$, tornando os cálculos muito mais custosos e, além disso, implica que o potencial de redução relativo é maior, já que a 


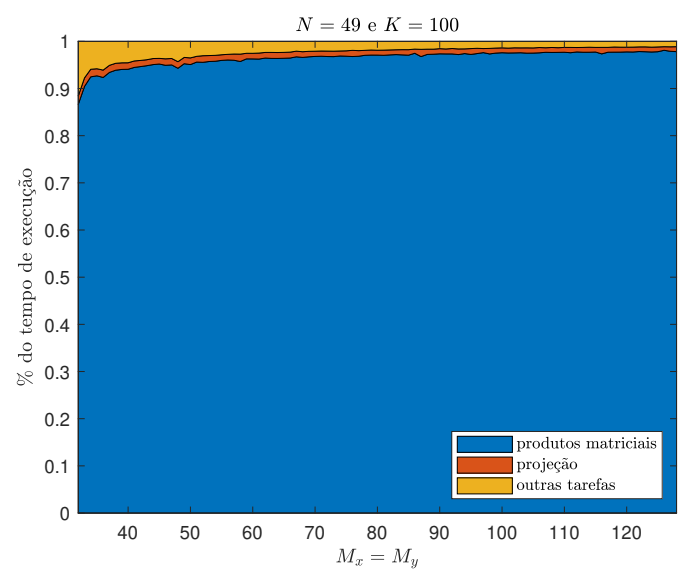

(a)

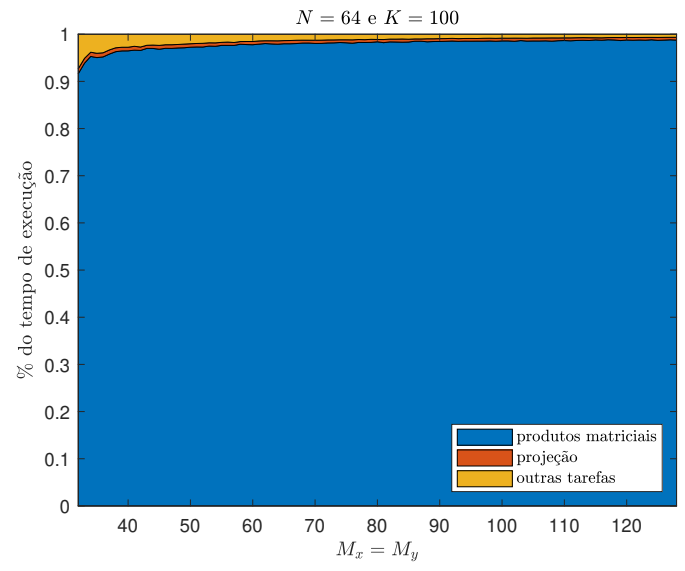

(c)

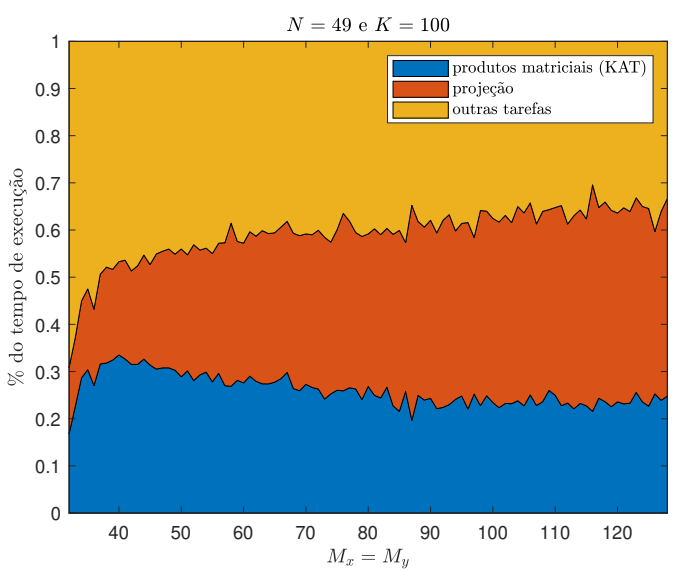

(b)

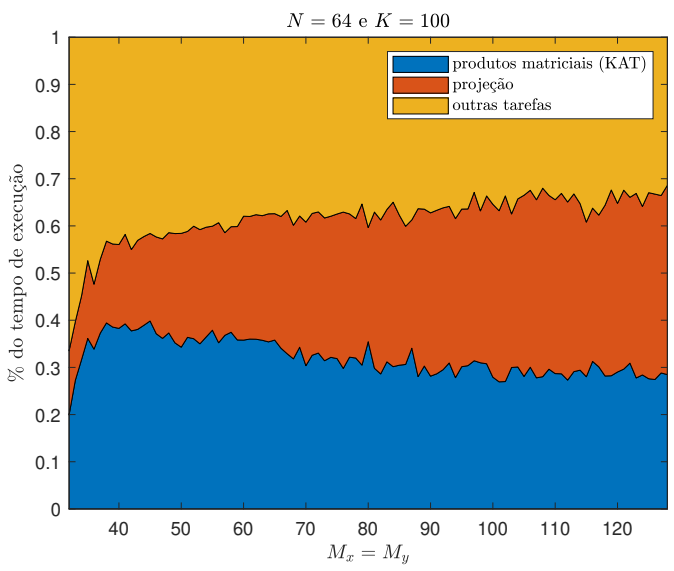

(d)

Figura 19 - Porcentagem do tempo de total de cálculo devida a cada uma das tarefas principais realizadas pelo método de ajuste de covariância reescrito como um problema do tipo BPDN para estimar uma imagem acústica em função do tamanho da imagem $M_{x}=M_{y}$. São mostrados dois casos com arranjos de geometria separável com $N=100$ e $N=64$. Todas as imagens possuíam uma distribuição com 30 fontes pontuais na frequência de $11 \mathrm{kHz}$ e $M_{x}=M_{y}=100$. A quantidade de iterações foi escolhida de forma que o algoritmo ainda não atingiu a convergência, executando todas as operações mencionadas a cada iteração.

CSM KAT diminui relativamente uma maior quantidade de cálculos que a KAT. Nota-se também que o padrão de fração constante de cálculos com o aumento do número de iterações observado na Figura 15 se manteve.

Na Figura 19 temos o mesmo padrão de aumento do tempo gasto com produtos matriz-vetor à medida que o tamanho da imagem aumenta observado na Figura 16. A aplicação da CSM KAT reduz consideravelmente o peso total dos produtos matriz-vetor para cerca de $25 \%$ do tempo total e notamos que a operação de projeção tende a ser a operação mais custosa com o aumento de $M$.

Por fim, na Figura 20, comparamos os tempos de execução do algoritmo com 


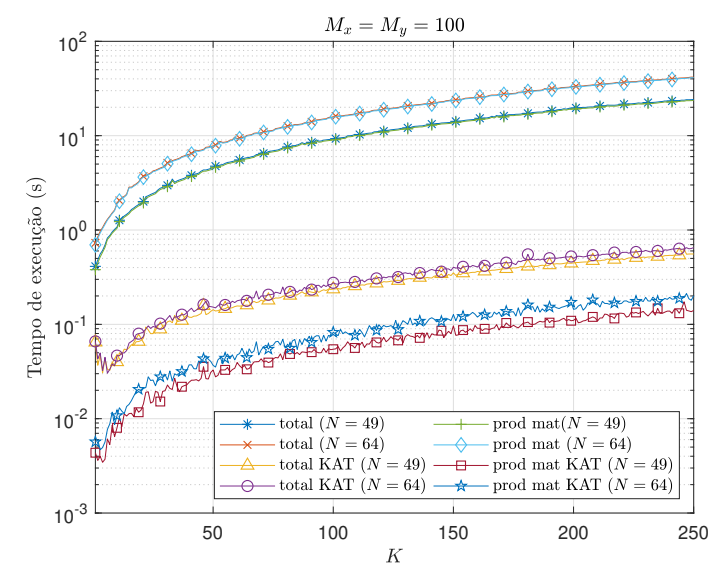

(a)

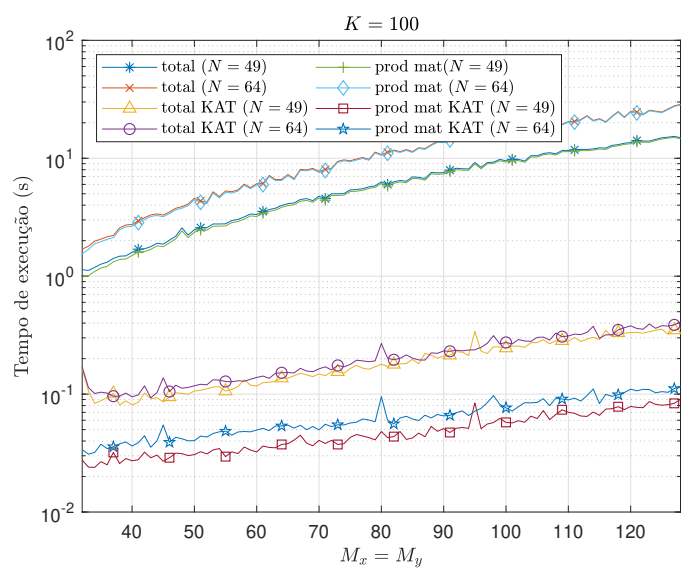

(b)

Figura 20 - Comparação dos tempos de execução para ajuste de covariância reescrito como um problema BPDN considerando as acelerações conseguidas com a aplicação das KATs direta e adjunta. Todas as imagens possuíam uma distribuição com 30 fontes pontuais na frequência de $11 \mathrm{kHZ}$ e correspondem exatamente aos casos analisados nas Figuras 18 e 19. A operação de projeção foi implementada em C e chamada pelo Matlab [5]. total se refer ao tempo total de cálculo e prod mat ao tempo gasto com os produtos matriz-vetor.

e sem o uso da KAT. A redução no número de operações executadas é da ordem de $\mathcal{O}\left(M N^{2}+M \log M\right)$ para $\mathcal{O}\left(\sqrt{M} N^{2}+M N+M \log M\right)$.

\subsection{Ajuste de covariância com regularização por variação total}

Como a regularização $\ell_{1}$ só é efetiva para casos em que a imagem é esparsa na sua representação canônica, convém apresentar um método alternativo que possa ser aplicado para imagens que apresentam fontes distribuídas. Uma possibilidade é reconstruir as imagens aplicando regularização por variação total $\left(\mathrm{TV}^{4}\right)[4]$.

De acordo com [8], a variação total isotrópica para uma imagem 2D é definida como

$$
\|\mathbf{Y}\|_{\mathrm{BV}}=\sum_{i, j} \sqrt{\left|\left[\nabla_{x} \mathbf{Y}\right]_{i, j}\right|^{2}+\left|\left[\nabla_{y} \mathbf{Y}\right]_{i, j}\right|^{2}}
$$

em que $\nabla_{x}$ e $\nabla_{y}$ são os operadores de primeira diferença nos eixos $x$ e $y$ com fronteiras periódicas dados por

$$
\begin{aligned}
& {\left[\nabla_{x} \mathbf{Y}\right]_{i, j}=[\mathbf{Y}]_{i+1, j}-[\mathbf{Y}]_{i, j}} \\
& {\left[\nabla_{y} \mathbf{Y}\right]_{i, j}=[\mathbf{Y}]_{i, j+1}-[\mathbf{Y}]_{i, j}}
\end{aligned}
$$

$i$ e $j$ são são os índices dos eixos $x$ e $y$, respectivamente; e $\|\mathbf{Y}\|_{\mathrm{BV}}$ é a chamada semi-norma de variação limitada ${ }^{5}$.

\footnotetext{
4 Do inglês total variation.

5 Do inglês bounded variation.
} 
Seja

$$
[\nabla \mathbf{Y}]_{i, j}=\left[\begin{array}{l}
{\left[\nabla_{x} \mathbf{Y}\right]_{i, j}} \\
{\left[\nabla_{y} \mathbf{Y}\right]_{i, j}}
\end{array}\right]
$$

o gradiente discreto de $\mathbf{Y}$ no pixel de índice $(i, j)$. Podemos entender $\nabla$ como um operador discreto que mapeia uma imagem $\mathbf{Y} \in \mathbb{R}^{M_{x} \times M_{y}}$ para o campo vetorial $\nabla \mathbf{Y} \in\left(\mathbb{R}^{2}\right)^{M_{x} \times M_{y}}$ (a última coluna e linha podem ser completadas com zeros para manter o mesmo tamanho da imagem), então $\|\mathbf{Y}\|_{\mathrm{BV}}=\|\operatorname{vec}\{|\nabla \mathbf{Y}|\}\|_{1}$, em que $|\nabla \mathbf{Y}| \in \mathbb{R}^{M_{x} \times M_{y}}$ é uma matriz cujos elementos são $\left[\mid \nabla \mathbf{Y}\left\|_{i, j}=\right\|[\nabla \mathbf{Y}]_{i, j} \|_{2}\right.$. Portanto, a regularização TV considera que o gradiente do sinal (ou imagem) é esparso, ao minimizar a norma $\ell_{1}$ de sua magnitude, ao invés de considerar o próprio sinal como esparso.

O problema de otimização para este caso fica

$$
\min _{\mathbf{Y}}\|\mathbf{Y}\|_{\mathrm{BV}}+\frac{\mu}{2}\|\mathbf{s}-\mathbf{A y}\|_{2}^{2} \quad \text { sujeito a }[\mathbf{Y}]_{i, j} \geq 0
$$

Como a variação total mede as descontinuidades da imagem e sua minimização busca a solução com gradiente mais esparso, então as imagens reconstruídas irão preservar bordas e fronteiras de maneira mais precisa do que minimização $\ell_{1}$ [68]. Isso significa que esse método se aplica melhor a imagens constantes por partes ou que variam monotonicamente, como no caso de fontes distribuídas, porém também é capaz de recuperar imagens esparsas.

Esta formulação foi criada inicialmente para aplicações de remoção de ruído em imagens por [69] e depois generalizada para problemas de reconstrução de imagens.

Para resolver esse tipo de problema, utilizaremos o solver TVAL3 [68] conforme sugerido por [8]. TVAL3 é capaz de resolver o problema (6.48) e variações para um sinal qualquer $\mathbf{y} \in \mathbb{C}^{M}$ ou $\mathbb{R}^{M}$ e é capaz de lidar com a restrição de não negatividade.

O solver TVAL3 implementa um método de multiplicadores de Lagrange aplicado a uma função lagrangiana aumentada específica. A função lagrangiana é minimizada de maneira alternada e utiliza um passo do método do gradiente descendente (ou gradiente projetado, no caso de restrição de não negatividade) por iteração.

A maior parte da complexidade do algoritmo se deve aos produtos do tipo Ax e $\mathbf{A}^{H} \mathbf{x}$ requeridos nas aplicação do método do gradiente para atualizar a direção do gradiente a cada iteração [68]. Essa característica encoraja o uso da CSM KAT que, além de reduzir o custo de memória em algumas ordens de magnitude, também irá reduzir consideravelmente o tempo de cálculo.

Na Figura 21 observamos a aceleração conseguida com a aplicação da CSM KAT em dois testes: variando a quantidade de iterações numa mesma imagem fixada; e variando o tamanho da imagem mantendo a quantidade de iterações fixa. 


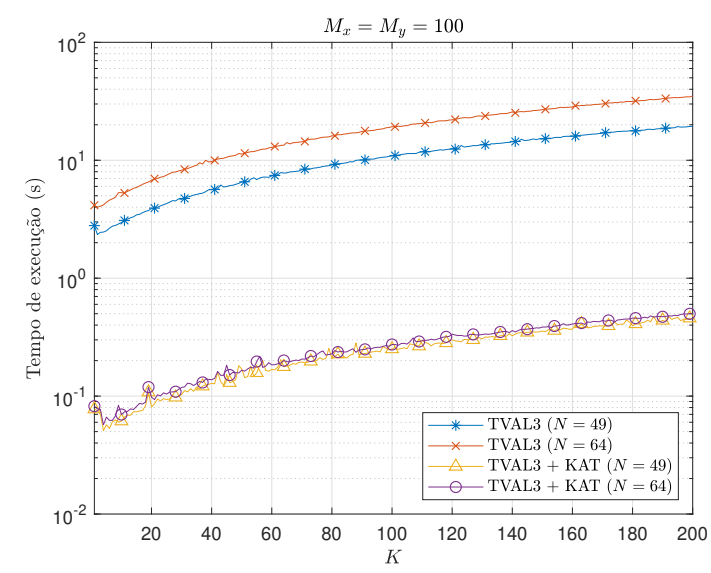

(a)

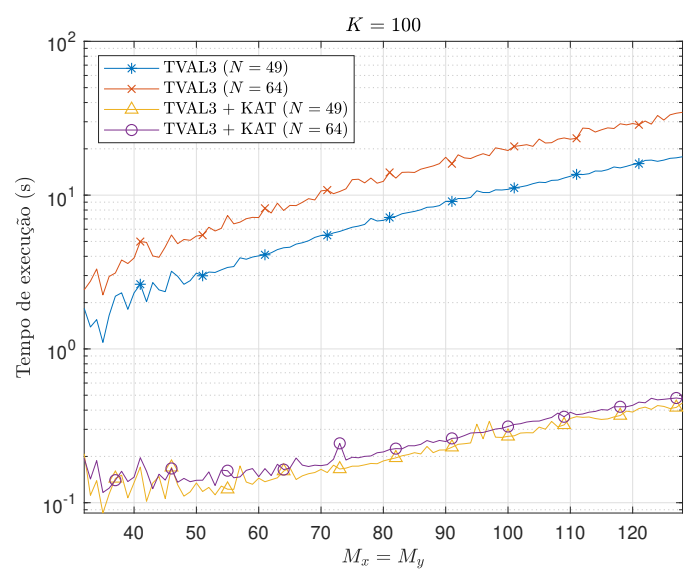

(b)

Figura 21 - Comparação dos tempos de execução para TVAL3 considerando as acelerações conseguidas com a aplicação das CSM KATs direta e adjunta. Todas as imagens possuíam uma distribuição com 10 fontes pontuais na frequência de $11 \mathrm{kHz}$ e $K$ representa a quantidade de iterações.

\subsection{Ajuste do sinal com regularização por variação total}

O imageamento por regularização TV para o problema de ajuste da matriz de covariância foi proposto por $[17,16]$ fazendo o uso do pacote TVAL3. Já o autor de [40] resolveu o problema convexo

$$
\min _{\mathbf{F}}\|\nabla \mathbf{F}\|_{2} \quad \text { sujeito a } \mathbf{V}_{y} \mathbf{F} \mathbf{V}_{x}^{T}=\mathbf{X}
$$

utilizando o pacote CVXPY [70, 71], que se mostrou o mais lento dentre os analisados mesmo usando a decomposição da KAT para reduzir o tamanho das matrizes envolvidas, porém não aproveitando a aceleração da KAT, já que o pacote não depende apenas de produtos por $\mathbf{V}^{H}$ e $\mathbf{V}$.

Como extensão do problema resolvido em [40], propomos resolver o problema TV diretamente no sinal $\mathbf{f}$ :

$$
\min _{\mathbf{F}}\|\mathbf{F}\|_{\mathrm{BV}} \quad \text { sujeito a } \mathbf{x}=\mathbf{V f}
$$

ou, no caso em que existe ruído de medida,

$$
\min _{\mathbf{F}}\|\mathbf{F}\|_{\mathrm{BV}}+\frac{\mu}{2}\|\mathbf{x}-\mathbf{V f}\|_{2}^{2}
$$

Ambos os problemas (6.50) e (6.51) podem ser resolvidos por TVAL3 mesmo $\mathbf{F}$ sendo uma matriz de números complexos. O operador $\nabla$, conforme seção anterior, mapeia uma imagem $\mathbf{Y} \in \mathbb{R}^{M_{x} \times M_{y}}$ para o campo vetorial $\nabla \mathbf{Y} \in\left(\mathbb{R}^{2}\right)^{M_{x} \times M_{y}}$. No entanto, TVAL3 também é capaz de lidar com $\nabla$ mapeando uma imagem $\mathbf{F} \in \mathbb{C}^{M_{x} \times M_{y}}$ para um campo vetorial $\nabla \mathbf{F} \in\left(\mathbb{C}^{2}\right)^{M_{x} \times M_{y}}$. 


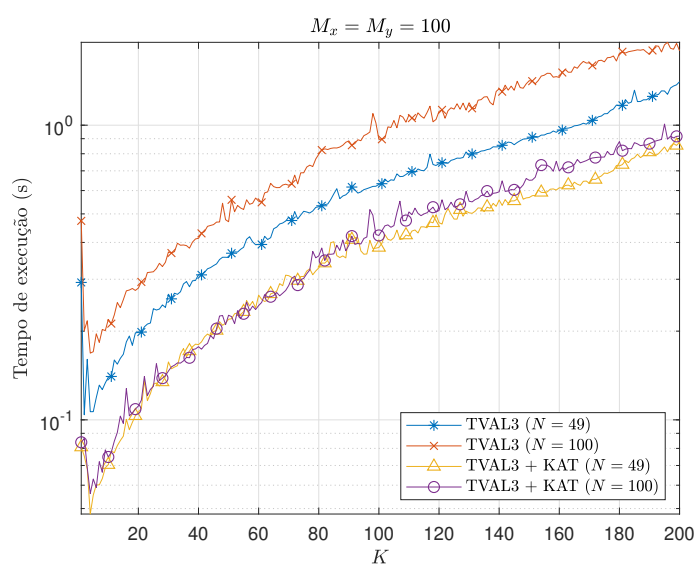

(a)

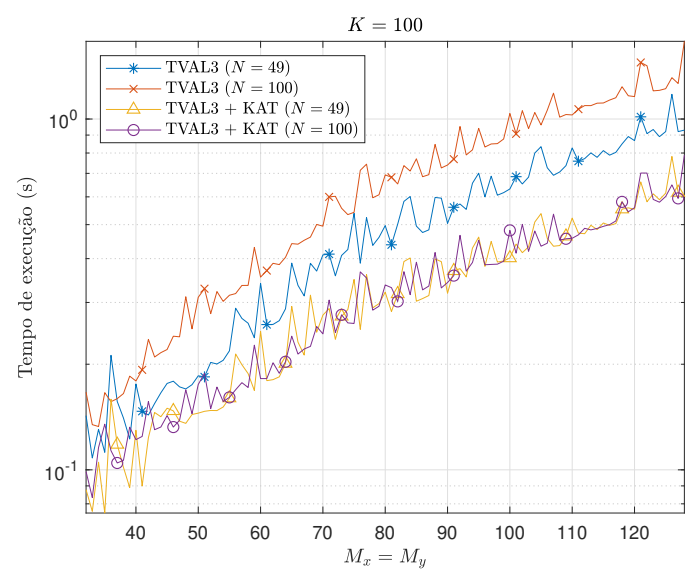

(b)

Figura 22 - Comparação dos tempos de execução para TVAL3 considerando as acelerações conseguidas com a aplicação das KATs direta e adjunta. Todas as imagens possuíam uma distribuição com 10 fontes pontuais na frequência de $11 \mathrm{kHz}$ e $K$ representa a quantidade de iterações.

O uso de TVAL3 permite também a aceleração dos cálculos utilizando as KATs direta e adjunta. Podemos observar a diferença nos tempos de execução com e sem KAT na Figura 22.

\subsection{Métodos de coordenadas descendentes}

As técnicas discutidas anteriormente para estimar imagens acústicas envolvem algoritmos gulosos ou a solução iterativa de algum problema de otimização. Os algoritmos gulosos (OMP e MP, por exemplo) tendem a requerer menor complexidade computacional que algoritmos de otimização, como SPGL1 e TVAL3, e são bons candidatos para implementações práticas em hardware [72].

Os autores de [72] observaram que os algoritmos de coordenadas descendentes (CD) são de baixa complexidade quando os sinais são esparsos e desenvolveram algoritmos baseados em iterações CD para problemas de otimização dos tipos $\ell_{2} \ell_{1}$ e $\ell_{2} \ell_{0}$. Essas iterações são de um tipo especial chamado de coordenadas descendentes dicotômicas $\left(\mathrm{DCD}^{6}\right)$ [73] que minimizam o uso de multiplicações e fornecem algoritmos altamente desejáveis pra aplicações práticas.

Algoritmos de coordenadas descendentes tentam encontrar a solução de um problema de otimização de várias variáveis minimizando uma variável de cada vez. A ideia central é minimizar uma coordenada a cada iteração (de forma exata ou não) ao invés de minimizar a função custo inteira a cada iteração.

Os autores de [72] também observaram que o algoritmos gulosos baseados em

6 Do inglês dichotomous coordinate descent. 
homotopia $\ell_{1}$ possuem alta precisão e baixa complexidade quando comparados com outros métodos de solução. De acordo com [52], resolver o problema de BP

$$
\min _{\mathbf{f}}\|\mathbf{f}\|_{1}, \quad \text { sujeito a } \mathbf{x}=\mathbf{V f}
$$

tradicionalmente envolve a solução de um sistema linear completo a cada iteração no caso denso. A homotopia $\ell_{1}$ é mais vantajosa no caso esparso pois começa com a solução $\mathbf{f}=\mathbf{0} \mathrm{e}$ vai adicionando e removendo elementos do suporte, atingindo a solução em poucos passos. Podemos comparar o comportamento do algoritmo de homotopia com o comportamento do OMP, porém capaz de remover elementos do suporte ao invés de apenas adicionar.

O método de homotopia $\ell_{1}$ envolve a solução em sequência de vários problemas de otimização do tipo

$$
\min _{\mathbf{f}} \frac{1}{2}\|\mathbf{x}-\mathbf{V f}\|_{2}^{2}+\lambda\|\mathbf{f}\|_{1}
$$

para diferentes valores do parâmetro de regularização $\lambda$ [52]. Como os algoritmos DCD são de natureza iterativa, [72] utiliza a estimativa atual da solução como inicialização da próxima iteração da homotopia (a chamada inicialização morna) para reduzir a complexidade, já que menos iterações DCD serão necessárias para atingir a solução. De modo que algoritmos gulosos baseados em homotopia com uso de iterações DCD serão de baixa complexidade e alta precisão. Essas mesmas vantagens se aplicam a homotopia $\ell_{0}$.

Sendo assim, estenderemos o uso dos algoritmos eficientes baseados em iterações DCD e homotopia de [72] para estimação de imagens acústicas. Além disso, apresentamos um novo algoritmo baseado em CD que usa a estrutura separável do arranjo em sua derivação e KAT direta-adjunta para aceleração dos cálculos.

Vamos descrever a seguir o algoritmo $\ell_{2} \ell_{0}$ de [72] que explora homotopia para regularização $\ell_{0}$. Queremos minimizar a função custo

$$
J_{\lambda}(\mathbf{f})=\frac{1}{2}\|\mathbf{x}-\mathbf{V f}\|_{2}^{2}+\lambda\|\mathbf{f}\|_{0}
$$

Seja $\mathcal{I}$ um conjunto de índices que representa o suporte de f. Quando $\lambda$ é grande, o segundo termo domina e a solução tende a $\mathcal{I}=\varnothing$, portanto a condição inicial do algoritmo é $\lambda$ grande e suporte vazio. Definimos $\lambda_{\max }$ como o mínimo valor de $\lambda$ para o qual ainda temos $\mathcal{I}=\varnothing$, então iniciamos $\lambda=\lambda_{\max }$ e o atualizamos no início de cada iteração de homotopia de acordo com a regra $\lambda \leftarrow \gamma \lambda$, em que $\gamma \in[0,1)$ é escolhido próximo da unidade.

Uma vez fixados $\lambda$ e $\mathcal{I}$, como ocorre no início de uma iteração da homotopia, minimizar $J_{\lambda}(\mathbf{f})$ é equivalente a minimizar apenas o primeiro termo da função custo, ou seja, resolver um problema de mínimos quadrados no suporte. Ao fazer isso, mantemos o tamanho do problema de otimização pequeno a cada iteração e conseguimos baixa complexidade [72]. 
Depois de resolvido o problema de mínimos quadrados, o algoritmo decide quais elementos adicionar ou remover do suporte. Essas decisões são feitas conforme o comportamento da função custo.

Sejam $\mathbf{b}=\mathbf{V}^{H} \mathbf{x}, \mathbf{R}=\mathbf{V}^{H} \mathbf{V}, \mathbf{c}=\mathbf{b}-\mathbf{R} \mathbf{f}$ e $\mathbf{e}_{t}$ o vetor cujo t-ésimo elemento é igual à unidade e todos os outros elementos são zero. A regra para adicionar elementos no suporte é obtida notando que, para o t-ésimo elemento nulo (fora do suporte) de $\mathbf{f}$ denotado $f_{t}$, fazer $f_{t}=\alpha$ de forma que teremos $\tilde{\mathbf{f}}=\mathbf{f}+\alpha \mathbf{e}_{t}$ leva a uma variação $\Delta J_{\lambda}$ da função custo dada por

$$
\Delta J_{\lambda}=J_{\lambda}(\tilde{\mathbf{f}})-J_{\lambda}(\mathbf{f})=\frac{1}{2}|\alpha|^{2} R_{t, t}-|\alpha| \operatorname{Re}\left\{e^{-j \arg \alpha} c_{t}\right\}+\lambda .
$$

Da equação anterior encontramos o $\alpha$ que minimiza $\Delta J_{\lambda}$ e verificamos se esse valor reduz a função custo, isto é, se teremos $\Delta J_{\lambda}<0$. Isso resulta no fato de que adicionar o $t$-ésimo elemento ao suporte dado por

$$
t=\underset{k \in \mathcal{I}^{c}}{\arg \max } \frac{\left|c_{k}\right|^{2}}{R_{k, k}} \quad \text { sujeito a } \quad\left|c_{t}\right|^{2}>2 \lambda R_{t, t}
$$

reduz a função custo. Após adicionar um elemento ao suporte, devemos fazer $f_{t}=c_{t} / R_{t, t}$ e atualizar $\mathbf{c} \leftarrow \mathbf{c}-f_{t} \mathbf{R}_{, t}$. Ao fazer $\mathbf{f}=0$, obtemos da regra o valor inicial

$$
\lambda_{\max }=\frac{1}{2} \max _{k} \frac{\left|b_{k}\right|^{2}}{R_{k, k}} .
$$

De forma análoga, remover o $t$-ésimo elemento que faz parte do suporte de acordo com a regra

$$
t=\underset{k \in \mathcal{I}}{\arg \min }\left\{\frac{1}{2}\left|f_{k}\right|^{2} R_{k, k}+\operatorname{Re}\left\{x_{k}^{*} c_{k}\right\}\right\} \quad \text { sujeito a } \frac{1}{2}\left|f_{t}\right|^{2} R_{t, t}+\operatorname{Re}\left\{x_{t}^{*} c_{t}\right\}<\lambda
$$

também reduz a função custo. Para cada elemento removido, devemos atualizar $\mathbf{c} \leftarrow$ $\mathbf{c}+f_{t} \mathbf{R}_{:, t}$ e fazer $f_{t}=0$.

Note que, da forma que foram escritas, as regras incluem ou removem apenas um elemento no suporte por iteração, porém podemos também adotar a estratégia de incluir ou remover todos os elementos que obedecem às regras, ou seja, todos os que reduzem a função custo.

É importante dizer que uma iteração do algoritmo funciona na seguinte ordem: resolução do problema de mínimos quadrados; atualização de $\lambda$; remoção dos elementos do suporte; e adição de elementos ao suporte.

O problema de mínimos quadrados pode ser resolvido diretamente, por decomposição de Cholesky ou QR (de forma análoga ao OMP), por inversão recursiva de matriz, etc, porém [72] propõe o uso de iterações DCD para obter um algoritmo computacionalmente mais eficiente. 
Resolver o problema de mínimos quadrados corresponde a minimizar (6.54) com $\lambda$ fixado, logo é equivalente a minimizar

$$
J(\mathbf{f})=\|\mathbf{x}-\mathbf{V f}\|_{2}^{2}
$$

Adicionar um valor $\alpha$ a uma coordenada de $\mathbf{f}$ implica numa variação da função custo dada por

$$
\Delta J=J\left(\mathbf{f}+\alpha \mathbf{e}_{t}\right)-J(\mathbf{f}) .
$$

Expandimos a expressão anterior para chegar a

$$
\begin{aligned}
\Delta J & =\left[\mathbf{V}\left(\mathbf{f}+\alpha \mathbf{e}_{t}\right)-\mathbf{x}\right]^{H}\left[\mathbf{V}\left(\mathbf{f}+\alpha \mathbf{e}_{t}\right)-\mathbf{x}\right]-(\mathbf{V} \mathbf{f}-\mathbf{x})^{H}(\mathbf{V} \mathbf{f}-\mathbf{x}) \\
& =\alpha \mathbf{f}^{H} \mathbf{V}^{H} \mathbf{V} \mathbf{e}_{t}+\alpha^{*} \mathbf{e}_{t}^{H} \mathbf{V}^{H} \mathbf{V} \mathbf{f}+|\alpha|^{2} \mathbf{e}_{t}^{H} \mathbf{V}^{H} \mathbf{V} \mathbf{e}_{t}-\alpha^{*} \mathbf{e}_{t}^{H} \mathbf{V}^{H} \mathbf{x}-\alpha \mathbf{x}^{H} \mathbf{V} \mathbf{e}_{t} .
\end{aligned}
$$

Depois de alguma álgebra e de fazer $\mathbf{b}=\mathbf{V}^{H} \mathbf{x}, \mathbf{R}=\mathbf{V}^{H} \mathbf{V}$ e $\mathbf{c}=\mathbf{b}-\mathbf{R f}$, chegamos a

$$
\Delta J=|\alpha|^{2} R_{t, t}-2 \operatorname{Re}\left\{\alpha^{*} c_{t}\right\}
$$

De (6.61), temos que o valor $\alpha=c_{t} / R_{t, t}$ minimiza $\Delta J$ para um $t$ fixo. Isso significa que podemos resolver o problema de mínimos quadrados escolhendo o $\alpha$ que minimiza (6.61), atualizar $f_{t} \leftarrow f_{t}+\alpha$ e $\mathbf{c} \leftarrow \mathbf{c}-\alpha \mathbf{R}_{:, t}$ (o que corresponde a uma iteração de CD) e repetir o processo alternando os valores de $t$ ciclicamente.

A resolução do problema por iterações CD já possui complexidade mais baixa que outros métodos de pesquisa linear [57], porém a complexidade pode ser reduzida ainda mais quando utilizamos iterações DCD. Note que o uso de CD requer divisões e multiplicações de números complexos.

Já nas iterações DCD, os números são de ponto fixo com $M_{b}$ bits limitados a um intervalo $[-H, H]$. Se escolhermos $H$ como uma potência de dois, todas as operações de multiplicação e divisão são substituídas por simples deslocamentos de bits, tornando o algoritmo mais indicado para implementação em hardware. O tamanho do passo $\alpha$ é escolhido de forma ótima no caso $\mathrm{CD}$, já no DCD iniciamos $\delta=H$ e a cada iteração fazemos $\delta \leftarrow \delta / 2$, eliminando assim divisões. Além disso, como estamos interessados em sinais complexos, podemos atualizar cada iteração em quatro direções diferentes no plano complexo; para fazer isso basta definir um vetor

$$
\boldsymbol{\alpha}=\left[\begin{array}{llll}
\delta & -\delta & j \delta & -j \delta
\end{array}\right]
$$

A pesquisa linear exata (calculando $\alpha$ ótimo) consegue uma redução maior da função custo a cada iteração, porém a convergência da pesquisa linear inexata (como no caso do DCD com $\delta$ fixado) pode levar a uma convergência mais rápida [72]. Utilizamos a inicialização morna quando passamos de uma iteração da homotopia para a seguinte; 
fazendo isso, poucas iterações DCD são necessárias para obter uma solução [72] a cada iteração da homotopia.

Como nosso passo $\delta$ é fixado, pode acontecer de $\Delta J$ não diminuir para um $\delta$ específico, portanto teremos iterações nas quais a solução x do problema de mínimos quadrados não será atualizada, resultando numa iteração de custo computacional mínimo que requer apenas uma comparação. A condição de comparação é dada por $\Delta J<0$ ou

$$
\operatorname{Re}\left\{\alpha_{p} c_{t}^{*}\right\}>\frac{\delta^{2} R_{t, t}}{2},
$$

em que $p=0,1,2,3$. Note que estamos atualizando apenas uma direção no plano complexo por vez resultando em quatro atualizações para cada elemento no suporte. Já que existem iterações de sucesso, nas quais a solução é atualizada, e iterações de nas quais só é feita uma comparação, podemos definir um número máximo de iterações de sucesso $N_{u} \ll M$ para controlar a complexidade do algoritmo, já que a inicialização morna reduz muito a quantidade de iterações DCD necessárias para alta precisão [74].

Aplicar a homotopia $\ell_{0}$ combinada com as iterações DCD nos permite obter um algoritmo com complexidade comparável a de um método guloso com o MP porém com performance próxima a algoritmos de otimização convexa de alta performance [72]. Uma desvantagem desses algoritmos é o fato de dependerem de aplicações nas quais a matriz $\mathbf{R}=\mathbf{V}^{H} \mathbf{V}$ está disponível. De fato, o ganho na complexidade depende exatamente dessa característica. Nos casos em que $\mathbf{R}$ não está disponível, a complexidade computacional depende do cálculo de $\mathbf{R}$ em tempo real. Sabemos, dos capítulos anteriores, que a matriz $\mathbf{R}$ ocupa muito espaço na memória, de forma que trabalhar com sua representação explícita é impraticável já para problemas de tamanho médio. Portanto, deduziremos um novo algoritmo de homotopia $\ell_{0}$ para o cálculo de imagens acústicas envolvendo arranjos decomponíveis em produtos de Kronecker e veremos como a KAT direta-adjunta é capaz de reduzir significativamente a complexidade computacional do algoritmo.

\subsection{Homotopia para dicionários decomponíveis em produtos de Kronecker}

Vamos deduzir um novo algoritmo de homotopia $\ell_{0}$ que leva em conta a decomposição em produtos de Kronecker $\mathbf{V}=\mathbf{V}_{x} \otimes \mathbf{V}_{y}$. Considere o problema de minimizar a função custo

$$
J_{\lambda}(\mathbf{f})=\|\mathbf{x}-\mathbf{V f}\|_{2}^{2}+\lambda\|\mathbf{f}\|_{0}
$$

Para facilitar as derivações, vamos assumir $N_{x}=N_{y}=\sqrt{N}$ e $M_{x}=M_{y}=\sqrt{M}$.

Vale que

$$
\|\mathbf{x}-\mathbf{V f}\|_{2}^{2}=\left\|\operatorname{vec}^{-1}\{\mathbf{x}-\mathbf{V f}\}\right\|_{F}^{2}=\left\|\mathbf{X}-\mathbf{V}_{y} \mathbf{F} \mathbf{V}_{x}^{T}\right\|_{F}^{2},
$$


em que $\operatorname{vec}^{-1}\{\cdot\}$ desfaz a operação de vetorização obedecendo às dimensões originais das matrizes envolvidas, $\|\cdot\|_{F}$ denota a norma de Frobenius e usamos a identidade $\operatorname{vec}\left\{\mathbf{B Q A}^{T}\right\}=(\mathbf{A} \otimes \mathbf{B}) \operatorname{vec}\{\mathbf{Q}\}$. Podemos então reescrever a função custo na forma

$$
J_{\lambda}(\mathbf{F})=\left\|\mathbf{X}-\mathbf{V}_{y} \mathbf{F} \mathbf{V}_{x}^{T}\right\|_{F}^{2}+\lambda\|\mathbf{F}\|_{0},
$$

em que $\|\mathbf{F}\|_{0}$ representa o número de elementos não nulos na matriz $\mathbf{F}$.

Agora expandimos a função custo usando a propriedade da norma de Frobenius $\|\mathbf{A}\|_{F}=\sqrt{\operatorname{tr}\left\{\mathbf{A}^{H} \mathbf{A}\right\}}$ para obter

$$
J_{\lambda}(\mathbf{F})=\operatorname{tr}\left\{\left(\mathbf{X}-\mathbf{V}_{y} \mathbf{F} \mathbf{V}_{x}^{T}\right)^{H}\left(\mathbf{X}-\mathbf{V}_{y} \mathbf{F} \mathbf{V}_{x}^{T}\right)\right\}+\lambda\|\mathbf{F}\|_{0} .
$$

Depois de de alguma álgebra, chegamos a

$$
J_{\lambda}(\mathbf{F})=\|\mathbf{X}\|_{F}^{2}+\operatorname{tr}\left\{\mathbf{V}_{x}^{*} \mathbf{F}^{H} \mathbf{V}_{y}^{H} \mathbf{V}_{y} \mathbf{F} \mathbf{V}_{x}^{T}\right\}-\operatorname{tr}\left\{2 \operatorname{Re}\left\{\mathbf{X}^{H} \mathbf{V}_{y} \mathbf{F} \mathbf{V}_{x}^{T}\right\}\right\}+\lambda\|\mathbf{F}\|_{0} .
$$

Agora definimos as matrizes $\mathbf{R}^{X}=\mathbf{V}_{x}^{T} \mathbf{V}_{x}^{*}, \mathbf{R}^{Y}=\mathbf{V}_{y}^{H} \mathbf{V}_{y}$ e $\mathbf{C}=\mathbf{V}_{x}^{T} \mathbf{X}^{H} \mathbf{V}_{y}$; que são fixas durante a estimação de uma imagem acústica para uma frequência e podem ser calculadas previamente. Podemos reescrever a função custo em função dessas novas matrizes como

$$
J_{\lambda}(\mathbf{F})=\|\mathbf{X}\|_{F}^{2}+\operatorname{tr}\left\{\mathbf{R}^{X} \mathbf{F}^{H} \mathbf{R}^{Y} \mathbf{F}\right\}-2 \operatorname{Re}\{\operatorname{tr}\{\mathbf{C F}\}\}+\lambda\|\mathbf{F}\|_{0}
$$

em que usamos a propriedade de permutações cíclicas do traço.

Agora usamos a equação (6.69) para analisar o impacto de adicionar um valor $\alpha$ a $\mathbf{F}_{i, j}$ calculando a diferença

$$
\Delta J_{i, j}(\alpha)=J_{\lambda}\left(\mathbf{F}+\alpha \mathbf{e}_{i} \mathbf{e}_{j}^{H}\right)-J_{\lambda}(\mathbf{F}) .
$$

Depois de várias simplificações algébricas, chegamos a

$$
\begin{aligned}
\Delta J_{i, j}(\alpha)=|\alpha|^{2} \operatorname{tr}\left\{\mathbf{R}^{X} \mathbf{e}_{j} \mathbf{e}_{i}^{H} \mathbf{R}^{Y} \mathbf{e}_{i} \mathbf{e}_{j}^{H}\right\}+2 \operatorname{Re}\left\{\alpha\left(\mathbf{R}_{i,:}^{Y} \mathbf{F} \mathbf{R}_{:, j}^{X}\right)^{*}\right\} \\
-2 \operatorname{Re}\left\{\operatorname{tr}\left\{\alpha \mathbf{C e}_{i} \mathbf{e}_{j}^{H}\right\}\right\}+\lambda\left(\left\|\mathbf{F}+\alpha \mathbf{e}_{i} \mathbf{e}_{j}^{H}\right\|_{0}-\|\mathbf{F}\|_{0}\right),
\end{aligned}
$$

em que $\left(\left\|\mathbf{F}+\alpha \mathbf{e}_{i} \mathbf{e}_{j}^{H}\right\|_{0}-\|\mathbf{F}\|_{0}\right)=1$ se $\mathbf{F}_{i, j}=0$, ou seja, estamos adicionando um novo elemento ao suporte.

Se quisermos estudar o efeito de remover um elemento do suporte, devemos obrigatoriamente escolher $\alpha=-\mathbf{F}_{i, j}$ de forma que a diferença na função custo possuirá o mesmo formato, porém agora $\left(\left\|\mathbf{F}+\alpha \mathbf{e}_{i} \mathbf{e}_{j}^{H}\right\|_{0}-\|\mathbf{F}\|_{0}\right)=-1$ se $\mathbf{F}_{i, j} \neq 0$.

Podemos simplificar ainda mais a expressão obtida observando o formato dos produtos pelos vetores $\mathbf{e}_{i}$ e $\mathbf{e}_{j}$ :

$$
\begin{aligned}
\Delta J_{i, j}(\alpha)=|\alpha|^{2} \mathbf{R}_{j, j}^{X} \mathbf{R}_{i, i}^{Y}+2|\alpha| \operatorname{Re}\left\{e^{j \arg \alpha}\left[\left(\mathbf{R}_{i,:}^{Y} \mathbf{F} \mathbf{R}_{:, j}^{X}\right)^{*}-\mathbf{C}_{j, i}\right]\right\} \\
+\lambda\left(\left\|\mathbf{F}+\alpha \mathbf{e}_{i} \mathbf{e}_{j}^{H}\right\|_{0}-\|\mathbf{F}\|_{0}\right) .
\end{aligned}
$$


De posse dessa última expressão, podemos derivar as regras da homotopia para adicionar ou remover os elementos do suporte. $\mathrm{O}$ valor de $\arg \alpha$ que minimiza $\Delta J_{i, j}(\alpha)$ é $\arg \alpha=$ $-\arg \left\{\left(\mathbf{R}_{i,:}^{Y} \mathbf{F} \mathbf{R}_{:, j}^{X}\right)^{*}-\mathbf{C}_{j, i}\right\}$ que, ao ser substituído na equação anterior, leva a

$$
\Delta J_{i, j}(\alpha)=|\alpha|^{2} \mathbf{R}_{j, j}^{X} \mathbf{R}_{i, i}^{Y}+2|\alpha|\left[\left(\mathbf{R}_{i,:}^{Y} \mathbf{F} \mathbf{R}_{:, j}^{X}\right)^{*}-\mathbf{C}_{j, i}\right]+\lambda\left(\left\|\mathbf{F}+\alpha \mathbf{e}_{i} \mathbf{e}_{j}^{H}\right\|_{0}-\|\mathbf{F}\|_{0}\right) .
$$

Minimizar a expressão anterior em relação a $|\alpha|$ nos fornece

$$
|\alpha|=\frac{\left|\mathbf{C}_{j, i}-\left(\mathbf{R}_{i,:}^{Y} \mathbf{F} \mathbf{R}_{:, j}^{X}\right)^{*}\right|}{\mathbf{R}_{j, j}^{X} \mathbf{R}_{i, i}^{Y}} .
$$

Já substituímos o argumento de $\alpha$ que minimiza $\Delta J_{i, j}(\alpha)$, agora substituímos $|\alpha|$ para obter a diferença na função custo:

$$
\Delta J_{i, j}(\alpha)=-\frac{\left|\mathbf{C}_{j, i}-\left(\mathbf{R}_{i,:}^{Y} \mathbf{F} \mathbf{R}_{:, j}^{X}\right)^{*}\right|^{2}}{\mathbf{R}_{j, j}^{X} \mathbf{R}_{i, i}^{Y}}+\lambda\left(\left\|\mathbf{F}+\alpha \mathbf{e}_{i} \mathbf{e}_{j}^{H}\right\|_{0}-\|\mathbf{F}\|_{0}\right) .
$$

Além disso, como temos o argumento e o módulo de $\alpha$, podemos escrever

$$
\alpha=\frac{\mathbf{C}_{j, i}^{*}-\mathbf{R}_{i,:}^{Y} \mathbf{F R}_{:, j}^{X}}{\mathbf{R}_{j, j}^{X} \mathbf{R}_{i, i}^{Y}}
$$

De forma análoga ao algoritmo de homotopia $\ell_{0}$ de [72], dessas relações decidimos quais elementos adicionar ou remover do suporte com base em $\Delta J_{i, j}$.

Primeiro, para todo $(i, j)$ tal que $\mathbf{F}_{i, j} \neq 0$, calculamos $\Delta J_{i, j}\left(-\mathbf{F}_{i, j}\right)$ e resolvemos o simples problema de otimização

$$
\left(i^{*}, j^{*}\right)=\underset{i, j}{\arg \min } \Delta J_{i, j}\left(-\mathbf{F}_{i, j}\right)
$$

Se $\Delta J_{i^{*}, j^{*}}\left(-\mathbf{F}_{i^{*}, j^{*}}\right)<0$ fazemos $\mathbf{F}_{i^{*}, j^{*}}=0$, ou seja, removemos o elemento do suporte. Novamente podemos remover apenas um elemento por iteração da homotopia ou remover todos os que obedecem essa condição. Para remover mais de um elemento, basta repetir o problema de otimização nos elementos do suporte que ainda não foram excluídos, isto é, enquanto houver $\Delta J_{i^{*}, j^{*}}<0$.

Depois da etapa de remover elementos do suporte, decidimos quais elementos adicionar ao suporte. Para isso, calculamos $\alpha$ usando a equação (6.76) e depois calculamos $\Delta J_{i, j}(\alpha)$ para todos os $(i, j)$ tal que $\mathbf{F}_{i, j}=0$. Resolvemos outro problema de otimização simples dado por

$$
\left(i^{*}, j^{*}\right)=\underset{i, j}{\arg \min } \Delta J_{i, j}(\alpha)
$$

Se $\Delta J_{i^{*}, j^{*}}(\alpha)<0$, fazemos $\mathbf{F}_{i^{*}, j^{*}}=\alpha$.

A próxima etapa é calcular a solução $\mathbf{f}_{\mathcal{I}}$ no suporte identificado na iteração atual calculando $\mathbf{f}_{\mathcal{I}}=\mathbf{V}_{:, \mathcal{I}}^{\dagger} \mathbf{x}$, em que $\mathcal{I}$ é o suporte e $\mathbf{B}^{\dagger}$ denota a pseudo-inversa de uma matriz 
qualquer B, que não necessariamente precisa ser calculada diretamente. Podemos fazer esse cálculo usando algum método já discutido, como Cholesky ou DCD. O dicionário V pode ser usado diretamente ou podemos mapear os elementos do produto de Kronecker para salvar apenas $\mathbf{V}_{x}$ e $\mathbf{V}_{y}$ na memória e obter um algoritmo de baixo uso de memória em troca de alguns cálculos a mais.

Repetimos esses passos até que alguma condição de parada seja atingida. Essa condição pode ser relacionada ao parâmetro $\lambda$ da homotopia, um número máximo de iterações, uma esparsidade máxima ou algum erro residual.

Note que as expressões de $\alpha$ e de $\Delta J_{i, j}(\alpha)$ são calculadas de forma independente e a operação mais custosa computacionalmente é o cálculo de

$$
\hat{\mathbf{F}}_{i, j}=\mathbf{R}_{i,:}^{Y} \mathbf{F R}_{:, j}^{X}
$$

que é repetido para cada $(i, j)$ pelo menos uma vez (possivelmente até três vezes: uma ao remover elementos do suporte e duas ao adicionar) em apenas uma iteração de homotopia. Note que $\hat{\mathbf{F}}_{i, j}$ é o único produto matricial que aparece no meio das iterações do algoritmo, excetuando-se o passo de mínimos quadrados, todas as outras contas são operações escalares. Note também que, conforme o número de pixels da imagem acústica aumenta e para esparsidade baixa, o passo de mínimos quadrados não domina o tempo de cálculo; esse comportamento é análogo ao observado no algoritmo de OMP.

Se definirmos

$$
\hat{\mathbf{F}}=\mathbf{R}^{Y} \mathbf{F} \mathbf{R}^{X}=\mathbf{V}_{y}^{H} \mathbf{V}_{y} \mathbf{F} \mathbf{V}_{x}^{T} \mathbf{V}_{x}^{*},
$$

vemos imediatamente que $\hat{\mathbf{F}}$ é obtido ao se aplicar a KAT direta-adjunta a $\mathbf{F}$. De forma que podemos calcular $\mathbf{F}$ apenas uma vez por iteração e obter todos os escalares $\hat{\mathbf{F}}_{i, j}$ de uma só vez ao invés de recalculá-los para cada elemento a ser removido ou adicionado ao suporte.

Derivamos então um algoritmo de homotopia $\ell_{0}$ que usa a estrutura decomponível da matriz $\mathbf{V}$ para obter as regras usadas pra encontrar o suporte do sinal. Além disso, o algoritmo se vale da KAT direta-adjunta para acelerar os cálculos intermediários de forma que o custo computacional é limitado a uma KAT direta-adjunta por iteração. Existem outras operações realizadas no algoritmo porém são poucas multiplicações escalares e comparações.

A grande vantagem desse algoritmo comparado com a homotopia $\ell_{0}$ de [72] é o fato de ele não depender da matriz $\mathbf{R}=\mathbf{V}^{H} \mathbf{V}$ e armazenar somente as matrizes $\mathbf{V}_{\mathbf{x}}$ e $\mathbf{V}_{y}$. Esse ganho é, de fato, muito importante para implementação prática do algoritmo conforme exposto na Figura 12. O algoritmo de [72] foi projetado para aplicações nas quais $\mathbf{R}$ está disponível e sua baixa complexidade computacional depende exatamente deste fato. Quando $\mathbf{R}$ não está disponível, o custo computacional será dominado pelo cálculo de $\mathbf{R}$ e 
aumenta consideravelmente. No nosso caso, trocamos o custo de calcular $\mathbf{R}$ uma vez por uma KAT a cada iteração e reduzimos o uso de memória em várias ordens de magnitude.

Primeiro vamos analisar o impacto de utilizar a KAT direta-adjunta para calcular $\hat{\mathbf{F}}$ uma vez por iteração ao invés de calcular os $\hat{\mathbf{F}}_{i, j}$ conforme o suporte é atualizado. Para isso vamos considerar dois critérios de parada do algortimo: o primeiro consiste em parar o algoritmo quando a homotopia atingir um valor $\lambda_{\min }=\mu \lambda_{\max }$, em que $\lambda_{\max }$ (obtido de $(6.75)$ ao se fazer $\mathbf{F}=0)$ é o menor valor de $\lambda$ para o qual a homotopia ainda possui suporte vazio dado por

$$
\lambda_{\max }=\max _{i, j} \frac{\left|\mathbf{C}_{j, i}-\left(\mathbf{R}_{i,:}^{Y} \mathbf{F} \mathbf{R}_{:, j}^{X}\right)^{*}\right|^{2}}{\mathbf{R}_{i, j}^{X} \mathbf{R}_{i, j}^{Y}}
$$

e $\mu \in[0,1)$ geralmente é escolhido como um número próximo de zero; e o segundo critério é o número máximo de iterações de homotopia $T$.

Na Figura 23, observamos os tempos de cálculo com e sem o uso da KAT para situações que envolvem a variação do tamanho da imagem estimada e a esparsidade do sinal.

No caso da Figura 23a temos um critério de parada de $\lambda<\lambda_{\min }$, ou seja, o número de iterações $T$ da homotopia será um valor fixo determinado por $\gamma$ e o valor de $\lambda_{\min }$ e uma imagem com esparsidade fixa $K=10$. A aceleração observada é maior conforme o tamanho da imagem aumenta já que as matrizes envolvidas aumentam de tamanho e os produtos matriciais acelerados pela KAT representam a maior parte do tempo de execução do algoritmo. Note que a diferença entre um arranjo de $N=100$ sensores e um de $N=64$ é bem pequena, conforme discutido anteriormente para acelerações envolvendo o problema de estimar o sinal f e não a potência $\mathbf{y}$. Para uma imagem $128 \times 128$ observamos uma aceleração de duas ordens de magnitude.

$\mathrm{Na}$ Figura 23b temos o mesmo critério de parada da Figura 23a, porém com o tamanho da imagem acústica fixado e a esparsidade $K$ de $\mathbf{f}$ variando. Observe que o número de iterações $T$ da homotopia é fixo, de forma que a aceleração da KAT é fixa para cada iteração independentemente do valor de $K$. Essa curva comprova também que a maior parte do tempo de cálculo é gasto com os produtos matriciais acelerados pela KAT, já que as iterações de homotopia com KAT envolvem produtos de números complexos e buscas simples do máximo ou mínimo de uma matriz.

Já na Figura 23c, a tamanho da imagem e a esparsidade da solução são mantidas fixas e o número de iterações da homotopia varia. Como a KAT direta-adjunta possui uma capacidade de aceleração fixa a cada iteração de homotopia, já que o tamanho das matrizes é fixo, observamos uma razão de ganho menor que a observada no Figura 23a. No entanto, a KAT ainda se mostra muito vantajosa reduzindo o custo computacional em aproximadamente duas ordens de magnitude para o caso considerado. 


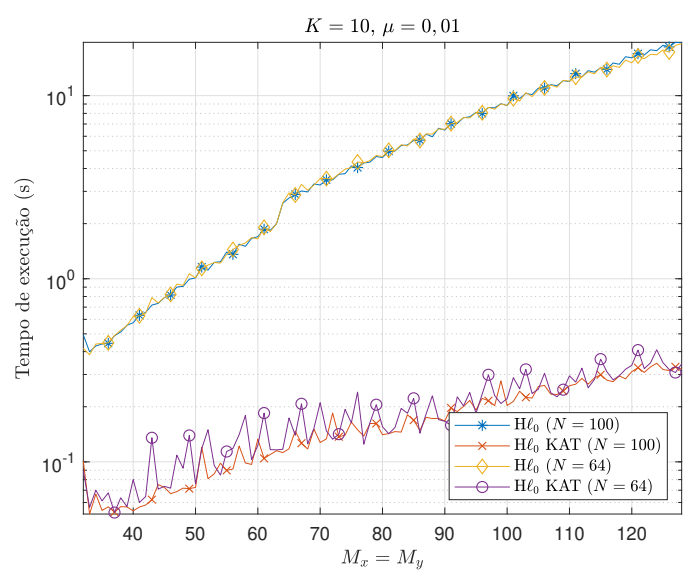

(a)

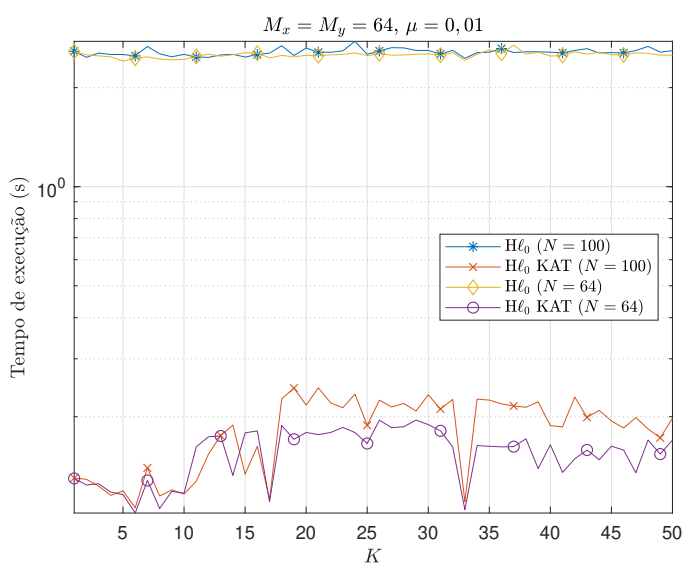

(b)

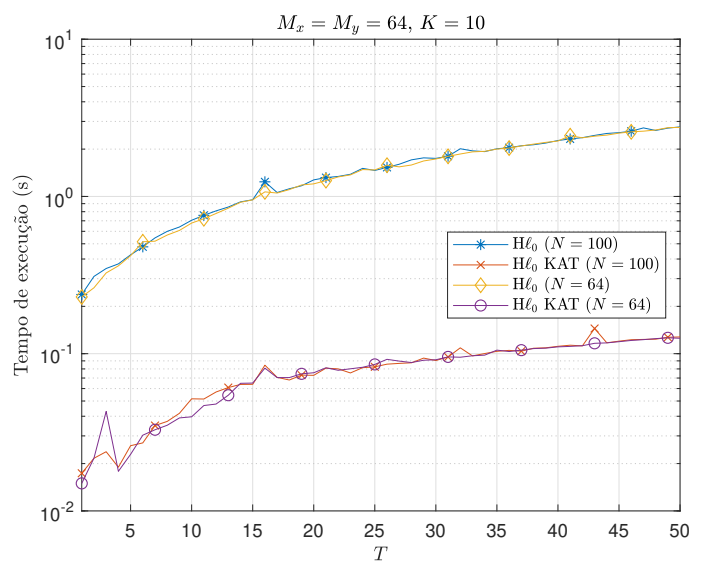

(c)

Figura 23 - Comparação dos tempos de execução para o algoritmo de homotopia $\ell_{0}$ para matriz decomponível em produtos de Kronecker considerando a aceleração conseguida com a KAT direta-adjunta. Todas as imagens foram calculadas na frequência de $10 \mathrm{kHz}$ e $\gamma=0,9$.

O uso da KAT direta-adjunta não só torna o custo de memória desses algoritmos muitas ordens de magnitude menor, tornando-os implementáveis, como também ameniza o custo extra computacional perdido por não sermos capazes de utilizar operações DCD como nos algoritmos de [72] que requerem a representação explícita da matriz $\mathbf{R}$ e são portanto proibitivos para aplicações de imagens acústicas. 


\section{Métodos similares à KAT}

A KAT foi proposta em [16] para acelerar estimação de imagens acústicas considerando arranjos com um geometria específica (separável) que permitia uma grande redução no custo computacional de produtos matriz-vetor.

Em [48], o autor propõe uma ideia similar à KAT para reduzir a complexidade computacional de problemas de mínimos quadrados associados a arranjos de sensores usados para aplicações de radar e sonar. A KAT explora a relação entre produtos de Kronecker e de Khatri-Rao; por outro lado, [48] explora a relação entre produtos de Kronecker e Schur-Hadamard para obter uma redução significativa no número de operações aritméticas necessárias para resolver um problema de mínimos quadrados.

Por outro lado, [75] propõe aplicar a transformada chirp-Z (CZT) para reduzir consideravelmente o número de operações requeridas num beamformer convencional. $\mathrm{O}$ método proposto requer arranjos planos ou volumétricos de geometria regular, isto é, arranjos que podem ser vistos como compostos por múltiplos segmentos paralelos de arranjos lineares uniformes.

Neste capítulo, analisaremos se a KAT pode ser aplicada nesses mesmos problemas e, em caso afirmativo, como ela se compara ao dois algoritmos.

\subsection{Transformada chirp-Z}

A saída de um beamformer convencional para uma frequência $\omega_{k}$ é dada por

$$
y\left(\mathbf{u}, \omega_{\mathbf{k}}\right)=\sum_{n=0}^{N-1} w_{n} x_{n}\left(\omega_{k}\right) e^{-j \frac{\omega_{k}}{c} \mathbf{u}^{T} \mathbf{p}_{n}},
$$

em que $x_{n}$ são os elementos de $\mathbf{x}\left(\omega_{k}\right)$ e $w_{n}$ são pesos ou ganhos aplicados aos sensores.

Se o arranjo possuir geometria linear uniforme com espaçamento entre elementos $d$ e $\theta$ for o ângulo de elevação, a saída do beamformer será

$$
y\left(\theta, \omega_{k}\right)=\sum_{n=0}^{N-1} w_{n} x_{n}\left(\omega_{k}\right) e^{-j \frac{\omega_{k}}{c} n d \sin \theta},
$$

portanto pode ser escrita como um polinômio complexo

$$
y\left(\theta, \omega_{k}\right)=\sum_{n=0}^{N-1} a_{n} z_{\theta}^{n},
$$

em que $z_{\theta}=e^{-j \frac{\omega_{k}}{c} d \sin \theta}$ e $a_{n}=w_{n} x_{n}\left(\omega_{k}\right)$. 
Como a largura de feixe é constante em função de $\sin \theta$, é usual escolher as direções $\theta$ uniformemente espaçadas no seno, ao invés de espaçadas uniformemente no próprio ângulo $\theta$ [76]. Portanto, para $M$ direções escolhemos ângulos $\theta_{m}$ tais que $\sin \theta_{m}=\sin \theta_{i}+m \Delta s$, $\Delta s=\left(\sin \theta_{f}-\sin \theta_{i}\right) /(M-1)$, em que $\theta_{i}$ e $\theta_{f}$ são respectivamente os ângulos inicial e final da região a ser observada.

A CZT é uma generalização da DFT usada para avaliar polinômios em contornos espirais no plano complexo dados por $z_{m}=A W^{-m}$, em que $A=A_{0} e^{-j 2 \pi \alpha_{0}}, W=W_{0} e^{j 2 \pi \omega_{0}}$ e $m=0,1, \ldots, M-1$. No caso do arranjo linear uniforme com amostragem uniforme no seno das direções $\theta$, basta fazer

$$
\begin{aligned}
A_{0} & =1, \quad \alpha_{0}=f_{k} \frac{d}{c} \sin \theta_{i} \\
W_{0} & =1, \quad \omega_{0}=f_{k} \frac{d}{c} \Delta s,
\end{aligned}
$$

em que $\omega_{k}=2 \pi f_{k}$. Agora substituímos $z_{\theta}$ por $z_{m}$ na equação (7.3) e chegamos a

$$
y\left(\theta_{m}, \omega_{k}\right)=W^{-\frac{m^{2}}{2}} \sum_{n=0}^{N-1} w_{n} A^{n} W^{-\frac{n^{2}}{2}} x_{n}\left(\omega_{k}\right) W^{-\frac{(m-n)^{2}}{2}}, m=0, \ldots, M-1 .
$$

Se definirmos $h(n)=w_{n} A^{n} W^{-\frac{n^{2}}{2}} x_{n}\left(\omega_{k}\right)$, a equação anterior é equivalente a uma convolução entre $h(n)$ e $v(n)=W^{\frac{n^{2}}{2}}$, permitindo a aceleração dos cálculos por FFT. Note que é necessário calcular a convolução apenas uma vez, bastando uma multiplicação por $W^{-\frac{m^{2}}{2}}$ para determinar a resposta para cada direção $\theta_{m}$.

Como as duas sequências a serem convoluídas são de tamanhos diferentes, precisamos usar zero padding e devemos escolher $L \geq N+M-1$. A CZT, então, requer duas FFTs de $L$ pontos para o cálculo da convolução entre $h(n)$ e $v(n)$.

Até agora, descrevemos a aceleração conseguida com o uso da CZT para um arranjo linear uniforme. Segundo [75], a aceleração pode ser explorada para arranjos planos e volumétricos com geometria regular, isto é, arranjos que podem ser vistos como compostos por múltiplos arranjos lineares uniformes.

Descrevemos agora o método rápido de [75] para beamforming. Considere um arranjo plano com geometria regular composto por $N$ microfones dispostos em $N_{y}$ linhas paralelas ao eixo $x \operatorname{com} N_{x}$ microfones cada tal que $N=N_{x} N_{y}$. Suponha que queiramos formar uma imagem com $M=M_{A} M_{E}$ direções tal que $M_{A}$ é o número de diferentes azimutes e $M_{E}$ é o número de diferentes elevações.

Seja $\mathbf{p}_{n}=\mathbf{p}_{i \ell}$ a posição do $n$-ésimo microfone escolhido como $\ell$-ésimo microfone na $i$-ésima linha, ou seja, estamos considerando um mapa $n=n(i, \ell)$. Seja $\Delta \mathbf{p}$ o espaçamento de dois microfones numa mesma linha, então

$$
\mathbf{p}_{i \ell}=\mathbf{p}_{i 0}+\ell \Delta \mathbf{p}
$$


em que $\mathbf{p}_{i 0}$ é a posição do primeiro elemento da $i$-ésima linha. Reescrever (7.1) em termos de $(i, l)$ leva a

$$
y\left(\mathbf{u}, \omega_{k}\right)=\sum_{i=0}^{N_{y}-1} \sum_{\ell=0}^{N_{x}-1} w_{i \ell} x_{i \ell}\left(\omega_{k}\right) e^{-j \frac{\omega_{k}}{c} \mathbf{u}^{T} \mathbf{p}_{i \ell}}
$$

Agora, consideramos apenas apenas a $i$-ésima linha do arranjo plano e substituímos (7.7) para chegar a

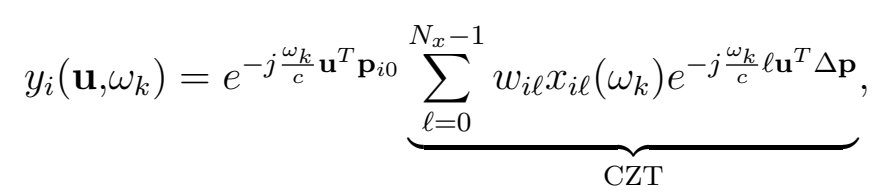

em que $y_{i}\left(\mathbf{u}, \omega_{k}\right)$ representa a operação de beamforming para uma arranjo linear uniforme multiplicada por uma constante complexa.

A parte destacada da equação (7.9) corresponde ao caso discutido anteriormente de CZT para um arranjo linear uniforme, porém com os azimutes distribuídos uniformemente no cosseno do ângulo $\phi$. Basta fazer

$$
\begin{aligned}
A_{0} & =1, \quad \alpha_{0}=\frac{\omega_{k}}{2 \pi c} \mathbf{u}_{0}^{T} \Delta \mathbf{p} \\
W_{0} & =1, \quad \omega_{0}=\frac{\omega_{k}}{2 \pi c} \Delta \mathbf{u}^{T} \Delta \mathbf{p},
\end{aligned}
$$

em que $\mathbf{u}_{0}$ e $\mathbf{u}_{M_{A}-1}$ são as direções correspondentes aos azimute inicial e final e

$$
\Delta \mathbf{u}=\frac{\mathbf{u}_{M_{A}-1}-\mathbf{u}_{1}}{M_{A}-1}
$$

é a separação entre cada azimute, que obteremos a soma presente em (7.9) de forma eficiente usando a CZT para as direções $\mathbf{u}_{0}, \ldots, \mathbf{u}_{M_{A}-1}$ correspondentes aos azimutes $\phi_{m}$, $0 \leq m<M_{A}$. Por fim, basta multiplicar o resultado da CZT pela constante complexa $e^{-j \frac{\omega_{k}}{c} \mathbf{u}_{m}^{T} \mathbf{p}_{i 0}}$ correspondente a cada azimute que teremos realizado beamforming para todos os azimutes numa única elevação para uma linha do arranjo plano. Necessitamos ainda formar os feixes para todas as elevações de interesse e depois somar todas as linhas do arranjo para obter o resultado final.

Para formar os feixes de todas as elevações para uma linha do arranjo, observamos que os feixes correspondentes a duas elevações diferentes, $\mathbf{u}_{0}$ e $\mathbf{u}_{1}$, num mesmo azimute só diferem por uma constante complexa. Temos

$$
y_{i}\left(\mathbf{u}_{0}, \omega_{k}\right)=e^{-j \frac{\omega_{k}}{c} \mathbf{u}_{0}^{T} \mathbf{p}_{i 0}} \sum_{\ell=0}^{N_{x}-1} w_{i \ell} x_{i \ell}\left(\omega_{k}\right) e^{-j \frac{\omega_{k}}{c} \ell \mathbf{u}_{0}^{T} \Delta \mathbf{p}}
$$

e, da equação (7.8),

$$
y_{i}\left(\mathbf{u}_{1}, \omega_{k}\right)=\sum_{\ell=0}^{N_{x}-1} w_{i \ell} x_{i \ell}\left(\omega_{k}\right) e^{-j \frac{\omega_{k}}{c}\left(\mathbf{u}_{0}+\delta \mathbf{u}\right)^{T} \mathbf{p}_{i \ell}}
$$

em que $\delta \mathbf{u}=\mathbf{u}_{1}-\mathbf{u}_{0}$. Substituir (7.7) leva a

$$
y_{i}\left(\mathbf{u}_{1}, \omega_{k}\right)=e^{-j \frac{\omega_{k}}{c} \mathbf{u}_{1}^{T} \mathbf{p}_{i 0}} \sum_{\ell=0}^{N_{x}-1} w_{i \ell} x_{i \ell}\left(\omega_{k}\right) e^{-j \frac{\omega_{k}}{c} \ell \mathbf{u}_{0}^{T} \Delta \mathbf{p}} e^{-j \frac{\omega_{k}}{c} \ell \delta \mathbf{u}^{T} \Delta \mathbf{p}} .
$$


Como as linhas do arranjo são paralelas ao eixo $x$ e escolhendo um azimute apropriado teremos $\mathbf{u}^{T} \Delta \mathbf{p}=0$, então

$$
y_{i}\left(\mathbf{u}_{1}, \omega_{k}\right)=e^{-j \frac{\omega_{k}}{c} \delta \mathbf{u}^{T} \mathbf{p}_{i 0}} y_{i}\left(\mathbf{u}_{0}, \omega_{k}\right)
$$

Portanto podemos formar diferentes feixes em um mesmo azimute realizando apenas multiplicações por uma constante complexa. Note que a soma que aparece na equação é a mesma da CZT, fazendo com que possamos calcular todos os feixes para uma linha do arranjo realizando apenas uma CZT e $M$ multiplicações complexas, depois basta repetir o processo para as $N_{y}$ linhas e somar todos os resultados. O cálculo por método direto requer a realização de uma soma dupla para cada feixe ou, equivalentemente, um produto matriz-vetor.

Assim, concluímos a descrição do método rápido de [75]. Mostraremos a seguir que a KAT não é aplicável a esse problema exato, pois as escolhas de direções (azimutes e elevações) necessárias para aplicação da CZT não produzem uma amostragem separável no espaço $U$. No entanto, podemos definir um caso análogo para KAT aplicada a beamforming convencional e comparar o desempenho computacional dos dois métodos com o mesmo arranjo e mesma quantidade de direções, porém com amostragem diferente no espaço $U$.

Considere, por exemplo, uma amostragem no espaço $U$ com $M=120$ direções de forma que $M_{A}=20$ e $M_{E}=6$, no caso da CZT, e $M_{x}=20$ e $M_{y}=6$ no caso da KAT. A Figura 24 mostra as amostragens no espaço $U$ correspondentes à KAT e CZT. Note que a amostragem da KAT foi tomada de forma uniforme nesse exemplo, porém qualquer amostragem separável também poderia ser aplicada. Além disso, a amostragem da KAT poderia ser escolhida de maneira a estar contida apenas na região visível. O ponto é que a CZT requer direções amostradas em circunferências arbitrárias que dependem das elevações escolhidas e as direções nesses circunferências devem obedecer a uma distribuição uniforme no cosseno do ângulo de azimute. Sendo assim, a CZT requer uma amostragem que não é separável, portanto a KAT não pode ser aplicada.

Podemos, mesmo assim, comparar os custos computacionais das duas transformadas utilizando um arranjo com o mesmo número de microfones e a mesma quantidade de direções. Note que o método de [75] é aplicável a arranjos planos regulares que também possuem a propriedade de separabilidade, porém possuem geometria mais restrita. $\mathrm{O}$ método da CZT também só é aplicável aos casos de beamforming convencional, portanto é adequado compará-lo com DAS acelerado por KAT. Como o processamento realizado em ambos os casos é o mesmo tipo de beamforming, devemos esperar resultados parecidos na resolução das imagens obtidas.

$\mathrm{O}$ método direto requer a realização do produto $\mathbf{V}^{H} \mathbf{x}$ que necessita de $O_{D}=$ $8(N-2) M$ operações reais incluindo somas e multiplicações ou $C_{D}=N M$ multiplicações 

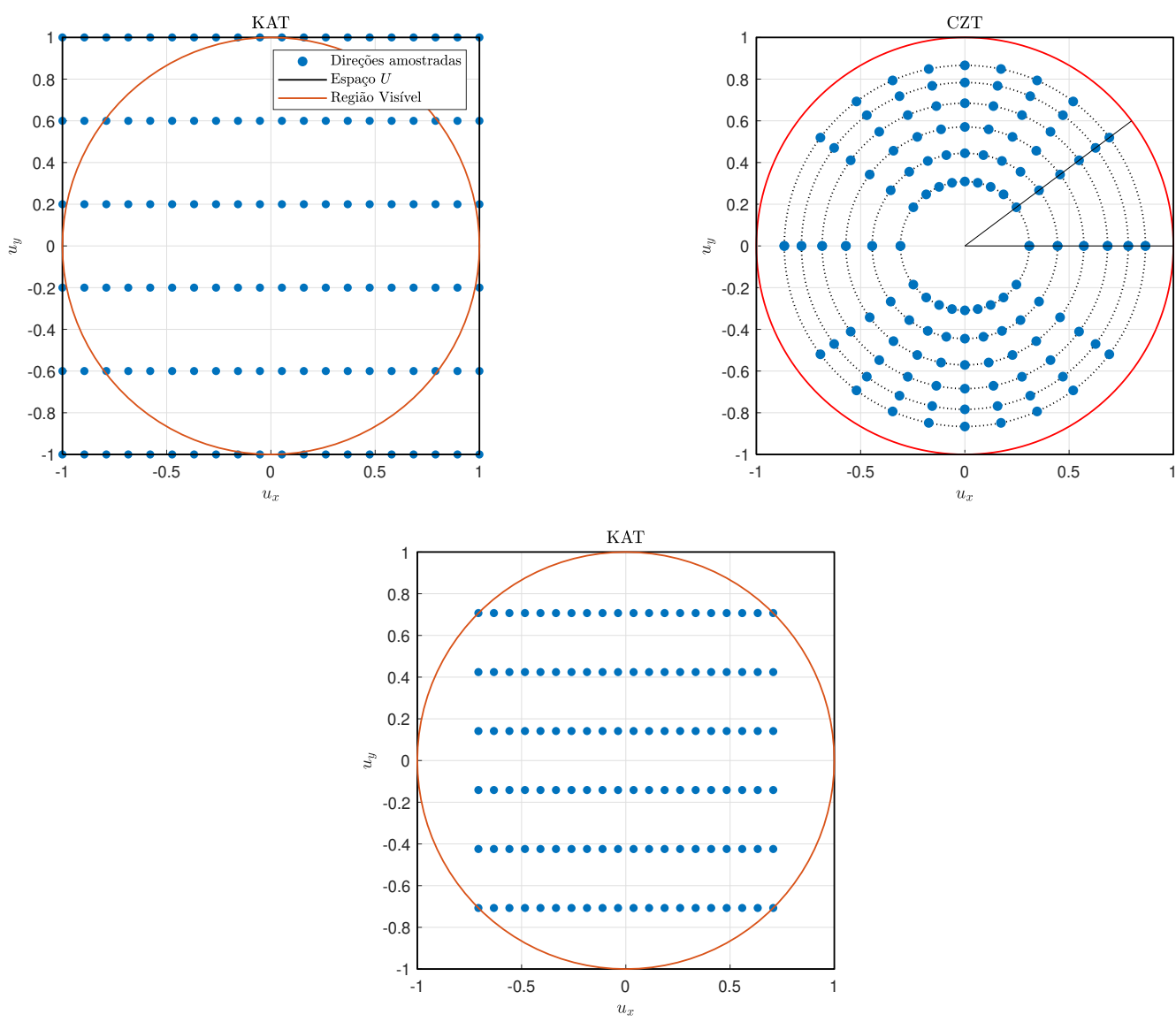

Figura 24 - Comparação de duas possíveis amostragens com o mesmo número de direções $M=120$ para KAT, uma restrita à região visível e outra espalhada por todo o espaço $U$, e uma possível amostragem para CZT. No caso da KAT, as direções foram distribuídas uniformemente nos retângulos $[-1,1]^{2}$ e $[-1 / \sqrt{2}, 1 / \sqrt{2}]^{2}$. No caso da CZT, as direções são distribuídas uniformemente no ângulo de elevação e uniformemente no cosseno do ângulo de azimute. Cada circunferência representa uma elevação e cada semi-reta partindo da origem representa um azimute.

complexas. O método da CZT requer [75]

$$
O_{C Z T}=6 N_{y}\left(N_{x}+L+M_{A}+M\right)+2 M\left(N_{y}-1\right)+10 N_{y} L \log _{2} L
$$

operações reais ou

$$
C_{C Z T}=N_{y}\left(N_{x}+L+M_{A}+M\right)+N_{y} L \log _{2} L
$$

multiplicações complexas, em que $L \geq M_{A}+N_{x}-1$ é uma potência de dois que representa o tamanho das FFTs envolvidas no cálculo da CZT. A aceleracão de DAS pela XKAT, por sua vez, requer

$$
O_{K A T}=4 N M_{x}+4 M N y+4 N M_{x}-2 N_{y} M_{x}+4 M N_{y}-2 M
$$

$\mathrm{Ou}$

$$
O_{K A T}=4 M_{y} N+4 N_{x} M+4 M_{y} N-2 M_{y} N_{x}+4 M N_{x}-2 M
$$


operações reais dependendo da ordem que as operações são tomadas. O número de multiplicações complexas correspondente será $C_{K A T}=N M_{x}+M N_{y}$ ou $C_{K A T}=M_{y} N+$ $N_{x} M$.

O método direto requer armazenamento da matriz $\mathbf{V}$ composta por $N M$ números complexos. Supondo precisão double, precisamos de

$$
M=16 N M
$$

bytes. Usar a CZT requer o armazenamento das constantes $A^{n} W^{\frac{n^{2}}{2}}$ que fazem parte da sequência $h(n)$, da sequência $W^{-\frac{n^{2}}{2}}$, da FFT da sequência $v(n)$ e das constantes complexas a serem multiplicadas para formação dos feixes de azimute e elevação. Portanto, a CZT requer

$$
M_{C Z T}=16\left(N_{x}+M_{A}+L+N_{y} M\right)
$$

bytes de armazenamento. Já para a XKAT basta armazenar as matrizes $\mathbf{V}_{x}$ e $\mathbf{V}_{y}$ que requerem

$$
M_{K A T}=16\left(N_{x} M_{x}+N_{y} M_{y}\right)
$$

bytes de memória.

A Figura 25 compara o número de multiplicações complexas e a quantidade total de operações reais entre a KAT e a CZT. O formato das curvas é praticamente o mesmo para ambos os casos. Os saltos observados na curva da CZT ocorrem exatamente nas mudanças de $L$, que é sempre tomado como uma potência de dois. Notamos que a KAT e a CZT possuem desempenho muito próximo em números de operações, com regiões nas quais a KAT é mais vantajosa e outras regiões nas quais a CZT custa menos. Na curva de memória, ainda na Figura 25, vemos que a KAT utiliza quase duas ordens de magnitude menos memória que a CZT.

Apesar do pequeno ganho no número de operações em relação à KAT, o método baseado em CZT de [75] se mostra menos vantajoso, já que só pode ser aplicado a uma geometria regular bem específica e somente para problemas de beamforming convencional. A KAT, além de permitir uma geometria mais geral, pode ser usada para acelerar outros métodos além de beamforming. Além disso, a KAT é mais simples de programar, uma vez que as matrizes $\mathbf{V}_{x}$ e $\mathbf{V}_{y}$ podem ser pré-computadas, e envolve apenas produtos matriciais. No entanto, o método da CZT pode ser aplicado a arranjos volumétricos nos quais a KAT não poderia ser aplicada, já que a distribuição de $u_{z}$ em função de $u_{x}$ e $u_{y}$ não seria uniforme não permitindo a decomposição da matriz $\mathbf{V}$ em produtos de Kronecker. 

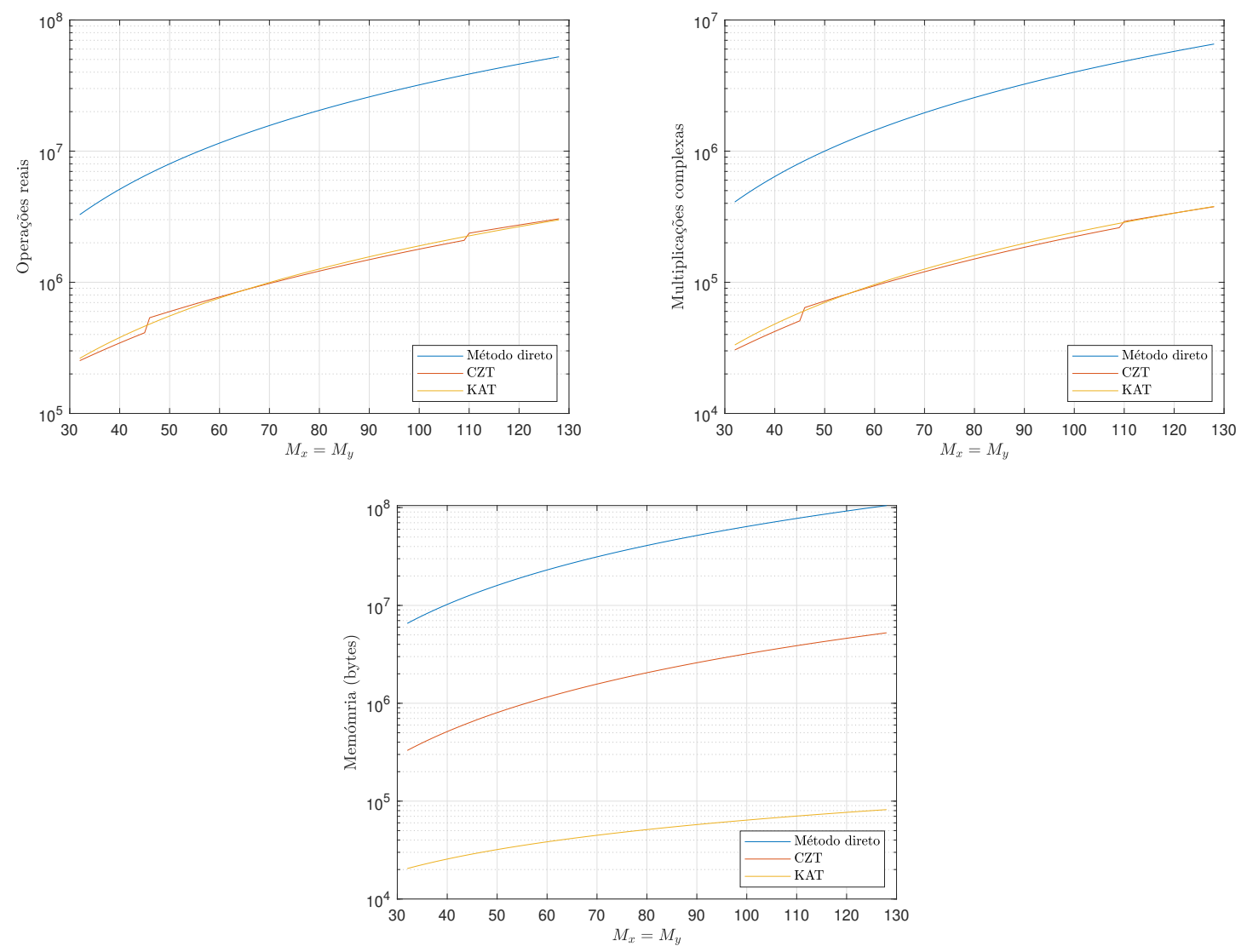

Figura 25 - Comparação do número de operações requeridas e memória em função da quantidade de direções pelos métodos XKAT e CZT com um mesmo arranjo de $N=400$ microfones arranjados numa grade $N_{x}=N_{y}=20$. Tomamos $M_{A}=M_{E}=M_{x}=M_{y}$ por simplicidade.

\subsection{Imagens acústicas por Least Squares acelerado com produto de Schur-Hadamard}

Nessa seção estendemos o método de [48] para o cálculo de imagens acústicas explorando as relações entre produtos de Khatri-Rao e Schur-Hadamard para obter uma redução significativa na quantidade de operações aritméticas necessárias para resolver um problema de mínimos quadrados

No cálculo de imagens acústicas, ao ignorar o ruído, temos

$$
\mathbf{S}=\mathbf{V} \mathbf{Y} \mathbf{V}^{H}=\mathbf{V} \mathbf{Y}\left(\mathbf{V}^{*}\right)^{T}
$$

em que $\mathbf{S}$ é a matriz espectral do arranjo, $\mathbf{Y}$ é a matriz espectral das fontes e $\mathbf{V}$ é a matriz diretora do arranjo. A imagem acústica consiste em obter uma estimativa $\hat{\mathbf{Y}}$ de $\mathbf{Y}$.

Assumindo fontes descorrelacionadas, $\hat{Y}$ é diagonal. Seja $L$ o número total de fontes pontuais diferentes de zero no campo das quais vamos assumir por hora que se conheça a direção de chegada. Vamos assumir também que $L<N^{2}$, ou seja, o número de fontes é menor que o número de microfones ao quadrado. 
Como $\hat{Y}$ é diagonal, então (7.23) é sobredeterminado, o que sugere resolver o problema de mínimos quadrados [48]

$$
\min _{\hat{\mathbf{Y}}}\left\|\mathbf{S}-\mathbf{V} \hat{\mathbf{Y}}\left(\mathbf{V}^{*}\right)^{T}\right\|_{F}^{2}
$$

sujeito à restrição que $\hat{\mathbf{Y}}$ é uma matriz diagonal.

A equação (7.23) pode ser reduzida quando $\mathbf{Y}$ possui uma diagonal esparsa:

$$
\mathbf{S}=\mathbf{V} \mathbf{Y} \mathbf{V}^{H}=\sum_{m=0}^{M-1} y_{m} \mathbf{v}\left(\mathbf{u}_{m}\right) \mathbf{v}^{H}\left(\mathbf{u}_{m}\right)=\sum_{l=0}^{L-1} y_{l} \mathbf{v}\left(\mathbf{u}_{l}\right) \mathbf{v}^{H}\left(\mathbf{u}_{l}\right)
$$

em que $y_{m}$ são os elementos da diagonal de $\mathbf{Y}$. Note que, na equação anterior, a enumeração $\mathbf{u}_{m}$ não necessariamente possui a mesma ordem de $\mathbf{u}_{l}$.

Definindo

$$
\begin{aligned}
& \overline{\mathbf{V}}=\left[\begin{array}{lll}
\mathbf{v}\left(\mathbf{u}_{1}\right) & \cdots & \mathbf{v}\left(\mathbf{u}_{L}\right)
\end{array}\right] \\
& \overline{\mathbf{Y}}=\operatorname{diag}\left(\left[\begin{array}{llll}
y_{0} & y_{1} & \cdots & y_{L-1}
\end{array}\right]\right),
\end{aligned}
$$

em que as direções correspondem às $L$ fontes, temos

$$
\mathbf{S}=\overline{\mathbf{V}} \overline{\mathbf{Y}}\left(\overline{\mathbf{V}}^{*}\right)^{T}
$$

e

$$
\min _{\overline{\mathbf{Y}}}\left\|\mathbf{S}-\overline{\mathbf{V}} \overline{\mathbf{Y}}\left(\overline{\mathbf{V}}^{*}\right)^{T}\right\|_{F}^{2}
$$

O problema (7.29) pode ser escrito de duas outras maneiras equivalentes. A primeira delas é

$$
\min _{\overline{\mathbf{Y}}}\left\|\operatorname{vec}\{\mathbf{S}\}-\left(\overline{\mathbf{V}}^{*} \otimes \overline{\mathbf{V}}\right) \operatorname{vec}\{\overline{\mathbf{Y}}\}\right\|_{2}^{2},
$$

em que $\otimes$ representa o produto de Kronecker, cuja solução é

$$
\operatorname{vec}\{\overline{\mathbf{Y}}\}=\left[\left(\overline{\mathbf{V}}^{*} \otimes \overline{\mathbf{V}}\right)^{H}\left(\overline{\mathbf{V}}^{*} \otimes \overline{\mathbf{V}}\right)\right]^{-1}\left(\overline{\mathbf{V}}^{*} \otimes \overline{\mathbf{V}}\right) \operatorname{vec}\{\mathbf{S}\}
$$

Como a matriz $\overline{\mathbf{Y}}$ é diagonal, resolver esse problema é altamente ineficiente, já que a maior parte dos elementos de $\overline{\mathbf{Y}}$ são iguais a zero, isto é, a matriz é esparsa.

A segunda maneira de reescrever o problema é

$$
\min _{\hat{\mathbf{Y}}}\left\|\operatorname{vec}\{\mathbf{S}\}-\left(\overline{\mathbf{V}}^{*} \odot \overline{\mathbf{V}}\right) \operatorname{vecd}\{\overline{\mathbf{Y}}\}\right\|_{2}^{2}
$$

em que $\odot$ denota o produto de Khatri-Rao, cuja solução é

$$
\operatorname{vecd}\{\overline{\mathbf{Y}}\}=\left[\left(\overline{\mathbf{V}}^{*} \odot \overline{\mathbf{V}}\right)^{H}\left(\overline{\mathbf{V}}^{*} \odot \overline{\mathbf{V}}\right)\right]^{-1}\left(\overline{\mathbf{V}}^{*} \odot \overline{\mathbf{V}}\right)^{H} \operatorname{vec}\{\mathbf{S}\} .
$$

As matrizes presentes nas soluções das duas maneiras de resolver o problema são grandes quando comparadas com a matriz $\overline{\mathbf{V}}$, com a segunda forma sendo mais 
eficiente que a primeira em termos de número de operações. A segunda forma pode ser implementada com o produto de Schur-Hadamard levando a uma redução significativa do custo computacional. Isso é feito explorando a relação

$$
(\mathbf{A} \odot \mathbf{B})^{H}(\mathbf{A} \odot \mathbf{B})=\left(\mathbf{A}^{H} \mathbf{A}\right) \circ\left(\mathbf{B}^{H} \mathbf{B}\right)
$$

em que o denota o produto de Schur-Hadamard [48]. A solução mais eficiente é, então,

$$
\operatorname{vecd}\{\overline{\mathbf{Y}}\}=\left[\left(\left(\overline{\mathbf{V}}^{*}\right)^{H} \overline{\mathbf{V}}^{*}\right) \circ\left(\overline{\mathbf{V}}^{H} \overline{\mathbf{V}}\right)\right]^{-1} \operatorname{vecd}\left\{\overline{\mathbf{V}}^{H} \mathbf{S} \overline{\mathbf{V}}\right\}
$$

Este método produz resultados melhores que resolver (7.31), porém é necessário conhecer previamente a direção das fontes. Além disso, o custo computacional é menor pois as matrizes são de dimensão reduzida: calcular o lado esquerdo de (7.34) requer $N^{2} L+$ $N^{2} L(L+1) / 2$ multiplicações; já o lado direito requer $(2 N+1) L(L+1) / 2$ multiplicações. Essa quantidade de operações já explora o fato das matrizes do tipo $\mathbf{A}^{H} \mathbf{A}$ que aparecem nas equações anteriores serem hermitianas e positivas definidas [48] e o fato de o produto de Schur-Hadamard ser fechado para matrizes hermitianas e positivas definidas [77].

Resolver (7.33) requer $N^{2} L+N^{2} L(L+1) / 2+\mathcal{O}\left(L^{3}\right)+L^{2} N^{2}+L N^{2}$ MACs complexas, em que o termo $\mathcal{O}\left(L^{3}\right)$ corresponde à inversão de matriz que aparece na equação. Já calcular (7.35) requer $(2 N+1) L(L+1) / 2+\mathcal{O}\left(L^{3}\right)+L N^{2}+L^{2} N+L^{2}$, em que o termo $\mathcal{O}\left(L^{3}\right)$ novamente corresponde à inversão de matriz.

Escolher um método para estimação das direções de chegada das fontes é um problema a ser resolvido e necessário para explorar a redução de complexidade computacional proporcionada pelo produto de Schur-Hadamard. Os métodos mais tradicionais para estimar direção de chegada são MUSIC [61] e ESPRIT [62], sendo aplicáveis apenas ao caso $L<N^{2}$. Ambos os métodos possuem resolução maior que um beamformer convencional, porém apresentam performance deficiente em situações de baixo SNR, poucos snapshots e fontes correlacionadas; além disso, métodos esparsos, como compressive beamforming alcançam resolução muito maior na estimação de direções de chegada [51].

Nesse mesmo contexto, podemos utilizar métodos de estimação esparsa ao invés de soluções de mínimos quadrados para encontrar uma estimativa $\hat{\mathbf{Y}}$. Por exemplo, o algoritmo de OMP aplicado ao cálculo de imagens acústicas efetivamente estima a direção de chegada numa grade definida de direções e resolve o problema (7.33) a cada iteração. Na próxima seção mostraremos como aplicar a relação de Schur-Hadamard em conjunto com a KAT para reduzir o custo computacional do algoritmo de OMP. Note que qualquer algoritmo que requeira produtos matriz-vetor da forma $\mathbf{A x}$ e $\mathbf{A}^{H} \mathbf{x}$ é passível de ser acelerado pela KAT, e qualquer algoritmo com problemas de mínimos quadrados da forma apresentada nessa seção pode ser acelerado pela relação de Schur-Hadamard, de forma que é apenas um exemplo de aplicação do método. 


\subsection{OMP acelerado por produto de Schur-Hadamard e KAT}

No seção 6.3, apresentamos o algoritmo OMP-X como uma forma iterativa de resolver aproximadamente o problema

$$
\min _{\mathbf{f}}\|\mathbf{f}\|_{0} \quad \text { sujeito a } \mathbf{x}=\mathbf{V f} .
$$

Podemos também aplicar OMP ao problema

$$
\min _{\mathbf{y}}\|\mathbf{y}\|_{0} \quad \text { sujeito a } \mathbf{s}=\mathbf{A y}
$$

Nesse caso, OMP recebe como entrada o sinal s, a matriz A e o nível de esparsidade $m$ do sinal ideal; e retorna como saída o vetor y que estima o sinal ideal. OMP inicializa o contador de iterações $\ell=1$, o conjunto de índices $\Lambda_{0}=\varnothing$ e o resíduo $\mathbf{r}_{0}=\mathbf{s}$.

Na iteração $\ell$, OMP encontrará a coluna $\mathbf{a}_{\lambda_{\ell}}$ de $\mathbf{A}$ mais correlacionada com o resíduo do sinal $\mathbf{r}_{\ell-1}$, resolvendo

$$
\lambda_{\ell}=\underset{j=1, \ldots, M}{\arg \max }\left|\left\langle\mathbf{r}_{\ell-1}, \mathbf{a}_{j}\right\rangle\right|,
$$

depois atualiza-se $\Lambda_{\ell}=\Theta_{\ell-1} \cup\left\{\lambda_{\ell}\right\}$. Então OMP achará uma aproximação para y com as colunas de $\mathbf{A}$ correspondentes ao conjunto de índices $\Lambda_{\ell}$ resolvendo o problema de mínimos quadrados

$$
\mathbf{y}_{t}=\underset{\mathbf{y}}{\arg \min }\left\|\mathbf{s}-\mathbf{A}_{\Lambda_{\ell}} \mathbf{y}\right\|_{2}
$$

Por fim, o algoritmo atualiza o resíduo

$$
\mathbf{r}_{\ell}=\mathbf{s}-\mathbf{A}_{\Lambda_{\ell}} \mathbf{y}_{\ell}
$$

e o processo é repetido se $\ell<L$.

O tempo de execução do algoritmo OMP pode ser reduzido explorando a solução acelerada de mínimos quadrados com produto de Schur-Hadamard e a KAT. Existem dois passos do algoritmo que possuem potencial de aceleração: o problema de otimização (7.38) e o problema de mínimos quadrados (7.39).

Seja $\mathbf{p}=\mathbf{A}^{H} \mathbf{r}$, então resolver (7.38) é equivalente a resolver

$$
\lambda_{t}=\underset{j=1, \ldots, M}{\arg \max }\left|\left\langle\mathbf{r}_{t-1}, \mathbf{a}_{j}\right\rangle\right|=\underset{j=1, \ldots, M}{\arg \max }\left|\left[\mathbf{p}^{H}\right]_{j}\right|=\underset{j=1, \ldots, M}{\arg \max }\left|\left[\mathbf{r}^{H} \mathbf{A}\right]_{j}\right| .
$$

Podemos desenvolver uma versão da KAT para o cálculo acelerado de p. Sabemos que a estrutura da matriz A, no caso de imagens acústicas com um arranjo de geometria separável, é dada por

$$
\mathbf{A}=\mathbf{V}^{*} \odot \mathbf{V}=\left(\mathbf{V}_{x} \otimes \mathbf{V}_{y}\right)^{*} \odot\left(\mathbf{V}_{x} \otimes \mathbf{V}_{y}\right)
$$


Ao aplicar a relação

$$
(\mathbf{A} \otimes \mathbf{B}) \odot(\mathbf{C} \otimes \mathbf{D})=\Xi[(\mathbf{A} \odot \mathbf{C}) \otimes(\mathbf{B} \odot \mathbf{D})]
$$

à equação anterior, temos

$$
\mathbf{A}=\left(\mathbf{V}_{x} \otimes \mathbf{V}_{y}\right)^{*} \odot\left(\mathbf{V}_{x} \otimes \mathbf{V}_{y}\right)=\Xi\left[\left(\mathbf{V}_{x}^{*} \odot \mathbf{V}_{x}\right) \otimes\left(\mathbf{V}_{y}^{*} \odot \mathbf{V}_{y}\right)\right]=\Xi\left(\tilde{\mathbf{V}}_{x} \otimes \tilde{\mathbf{V}}_{y}\right)
$$

em que $\Xi$ é uma matriz de permutação.

Assuma que $\mathbf{r}=\operatorname{vec}\{\mathbf{R}\}$, em que $\mathbf{R}$ é uma matriz de dimensão $N_{x} \times N_{y}$, e que $\mathbf{p}=\operatorname{vec}\{\mathbf{P}\}$, em que $\mathbf{P}$ é uma matriz $M_{x} \times M_{y}$. Temos

$$
\begin{aligned}
\operatorname{vec}\{\mathbf{P}\} & =\mathbf{A}^{H} \operatorname{vec}\{\mathbf{R}\} \\
& =\left[\Xi\left(\tilde{\mathbf{V}}_{x} \otimes \tilde{\mathbf{V}}_{y}\right)\right]^{H} \operatorname{vec}\{\mathbf{R}\} \\
& =\left(\tilde{\mathbf{V}}_{x} \otimes \tilde{\mathbf{V}}_{y}\right)^{H} \Xi^{T} \operatorname{vec}\{\mathbf{R}\} \\
& =\left(\tilde{\mathbf{V}}_{x} \otimes \tilde{\mathbf{V}}_{y}\right)^{H} \operatorname{vec}\{\mathbf{Z}\} \\
& =\left(\tilde{\mathbf{V}}_{x}^{H} \otimes \tilde{\mathbf{V}}_{y}^{H}\right) \operatorname{vec}\{\mathbf{Z}\} .
\end{aligned}
$$

Sabemos que

$$
\operatorname{vec}\left\{\mathbf{B Q A}^{T}\right\}=(\mathbf{A} \otimes \mathbf{B}) \operatorname{vec}\{\mathbf{Q}\}
$$

portanto

$$
\operatorname{vec}\{\mathbf{P}\}=\left(\tilde{\mathbf{V}}_{x}^{H} \otimes \tilde{\mathbf{V}}_{y}^{H}\right) \operatorname{vec}\{\mathbf{Z}\}=\operatorname{vec}\left\{\tilde{\mathbf{V}}_{y}^{H} \mathbf{Z} \tilde{\mathbf{V}}_{x}^{*}\right\}
$$

$\mathrm{Ou}$

$$
\mathbf{P}=\tilde{\mathbf{V}}_{y}^{H} \mathbf{Z} \tilde{\mathbf{V}}_{x}^{*}, \quad \operatorname{vec}\{\mathbf{Z}\}=\boldsymbol{\Xi}^{T} \operatorname{vec}\{\mathbf{R}\}=\boldsymbol{\Xi}^{T} \mathbf{r}
$$

A equação (7.52) é uma implementação da KAT adjunta que pode ser usada para acelerar o cálculo de (7.38).

Como $L \ll M$ e geralmente $N \ll M$, então o tamanho de $\mathbf{A}$ é muito grande mesmo para problemas de dimensões moderadas $[16,17]$. Sendo assim, o tempo de execução do algoritmo de OMP é dominado pelo cálculo de (7.38) que requer $L N^{2} M$ MACs complexas para $L$ iterações, e não por (7.39), que é $\mathcal{O}\left(\ell^{3}\right)+\mathcal{O}\left(2 N \ell^{2}\right)$.

O problema de mínimos quadrados (7.39) pode ser acelerado pela relação envolvendo o produto de Schur-Hadamard apresentada na seção 7.2. Como dito anteriormente, o método de [48] estendido para o cálculo de imagens acústicas necessita de uma maneira para se estimar a direção de chegada e a quantidade de sinais incidentes no arranjo. O algoritmo OMP já faz, de certa forma, essa estimativa de direções de chegada a cada iteração escolhendo a coluna de $\mathbf{A}$ mais correlacionada com o resíduo $\mathbf{r}$.

Para validar a aceleração obtida usando a KAT e a relação envolvendo os produtos de Schur-Hadamard, simulamos o algoritmo proposto num único núcleo de um processador 
Intel $2.0 \mathrm{GHz}$ com 100 realizações por ponto. As rotinas foram escritas em $\mathrm{C} / \mathrm{C}++\mathrm{e}$ chamadas no Matlab na forma de funções MEX.

Na Figura 26 observamos os tempos de execução de OMP com aplicação de aceleração por decomposição de Cholesky e aceleração pela relação envolvendo produtos de Schur-Hadamard derivada na seção anterior. Testamos os seguintes casos com e sem KAT: solução direta do problema de mínimos quadrados usando decomposição QR baseada em rotações de Householder; solução de mínimos quadrados de forma fechada usando a formulação de Khatri-Rao conforme equação (7.33); formulação de Schur-Hadamard conforme equação (7.35); e implementação rápida de OMP baseada em decomposição de Cholesky.

Fixamos o tamanho da imagem acústica em $M=4096$ e $M=10000$ para que tenhamos $M>N^{2}$ para os arranjos específicos de geometria separável com $N=49$ e $N=64$ microfones, ou seja, fixamos as dimensões da matriz $\mathbf{A}$ e então observamos o tempo de execução como uma função de $L$, a esparsidade de y. Os resultados são mostrados nas Figuras 26b e 26d. Note que, para $L$ pequeno, a aceleração obtida pela KAT é bem similar àquela obtida com a KAT combinada com produtos de Schur-Hadamard e com KAT combinada com decomposição de Cholesky, já que os produtos matriz-vetor dominam o tempo total de cálculo. Uma vez que $L$ aumenta, vemos que a combinação dos métodos de aceleração (Schur-Hadarmard combinado com KAT e Cholesky combinado com KAT) tem um ganho significativo quando comparada com o uso só da KAT, já que o passo de mínimos quadrados começa a responder por uma parcela maior do tempo de execução do algoritmo. Observamos uma aceleração pequena quando aplicamos apenas a relação de Schur-Hadamard ou a implementação rápida com decomposição de Cholesky, já que, como dito anteriormente, os produtos matriz-vetor tendem a dominar em aplicações de imagens acústicas.

Para investigar a aceleração como uma função de $M$, fixamos a esparsidade em $L=30$ e utilizamos os mesmo arranjos separáveis com $N=49$ e $N=64$ microfones. Os resultados são mostrados nas Figuras 26a e 26c. Vemos que o tempo de execução dos métodos acelerados que empregam a KAT estão crescendo a uma taxa muito menor que os outros métodos com uma aceleração de cerca de duas ordens de magnitude para $M_{x}=M_{y}=128$ e $N=64$. Conforme $M$ aumenta, as combinações de Schur-Hadamard com KAT e Cholesky com KAT tendem a se aproximar da curva que implementa apenas a KAT, já que para $L$ pequeno o passo de mínimos quadrados não é muito complexo e os produtos matriz-vetor dominam o tempo de execução.

Note que o método proposto baseado em produtos de Schur-Hadamard combinado coma KAT é competitivo e até ligeiramente mais rápido que a implementação rápida de OMP baseada em decomposição Cholesky, com e sem a KAT. No entanto, espera-se que o método de Cholesky seja mais rápido para casos de $L$ grande, porém o método de 


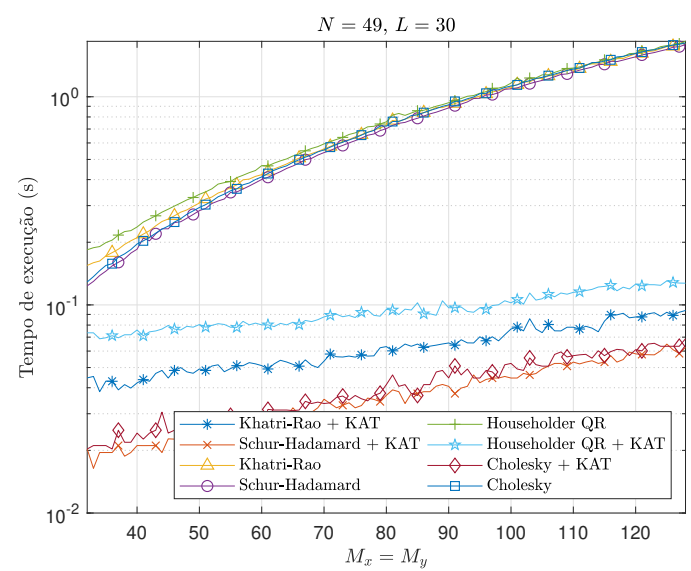

(a)

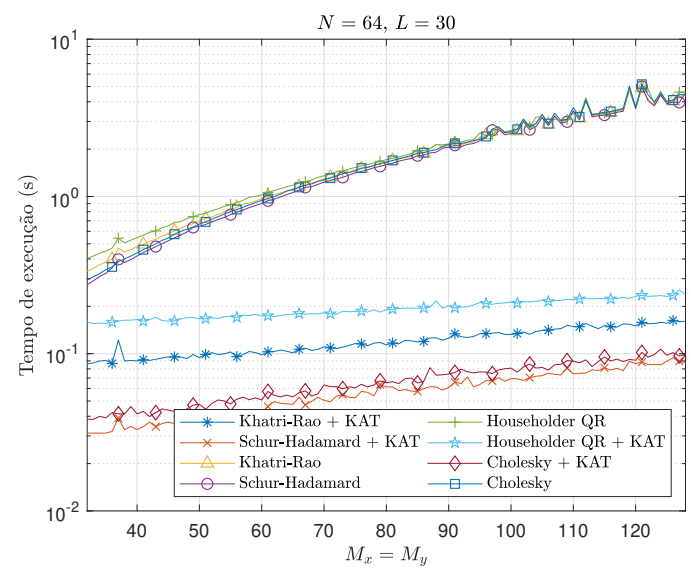

(c)

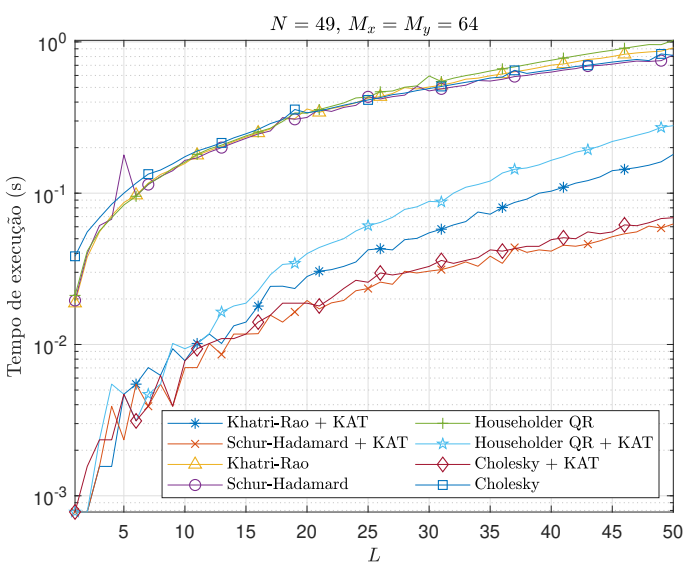

(b)

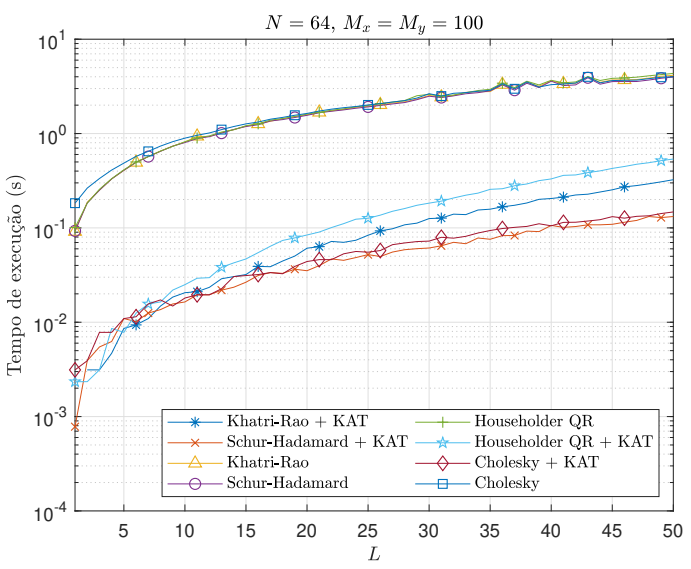

(d)

Figura 26 - Comparação do tempo de execução para OMP acelerado por Schur-Hadamard, as outras soluções baseadas em mínimos quadrados e a implementação rápida baseada em decomposição de Cholesky. Todas as rotinas foram escritas em $\mathrm{C} / \mathrm{C}++$ e chamadas no Matlab como funções MEX. São mostrados casos para dois arranjos separáveis, $N=49$ e $N=64$ e todas as imagens foram calculadas na frequência de $10 \mathrm{kHz}$.

Schur-Hadamard só é aplicável para $L<N^{2}$ e funciona melhor ainda para casos bem esparsos em que $L \ll N^{2}$. 



\section{Simulador para estimação de imagens acús- ticas}

Para que as análises de desempenho e comparações de diferentes algoritmos de estimação de imagens acústicas sejam feitas, foi desenvolvido um simulador no ambiente MATLAB.

O simulador é capaz de trabalhar com um arranjo de geometria qualquer, produz os sinais amostrados nos microfones do arranjo e calcula as principais matrizes usadas pelos algoritmos de estimação de imagens acústicas. O simulador também incorpora alguns métodos de estimação de imagens acústicas e mais métodos podem ser adicionados conforme a necessidade.

\subsection{Geometria do arranjo}

O simulador lida com a geometria do arranjo por meio de uma matriz que contém as coordenadas espaciais de cada microfone em uma de suas linhas. Sendo assim, é possível trabalhar com qualquer geometria arbitrária.

\subsection{Campo incidente e sinais nos microfones}

O simulador gera os sinais dos microfones a partir de uma representação em fontes pontuais em direções arbitrárias do campo incidente a ser definida pelo usuário. Conforme discutido no Capítulo 4, em campo distante podemos assumir a superposição de fontes pontuais. O usuário então decide um determinado padrão de fontes e o salva numa estrutura de dados similar a uma tabela; o simulador então lê a tabela de fontes, que possui todas as informações de direção de propagação, e gera o sinal nos microfones usando um modelo de ondas planas e atrasos.

Supondo uma distribuição de $M$ fontes, com direções correspondentes denotadas por $\mathbf{u}_{0}, \mathbf{u}_{1}, \ldots, \mathbf{u}_{M-1}$, dada por

$$
\mathbf{f}(t)=\left[\begin{array}{c}
f_{0}(t) \\
f_{1}(t) \\
\vdots \\
f_{M-1}(t)
\end{array}\right]
$$


o sinal da $m$-ésima fonte será recebido por todos os microfones, porém cada microfone receberá uma versão atrasada do mesmo sinal. O atraso dependerá da velocidade de propagação da onda $c$, da direção de propagação representada pelo vetor $\mathbf{u}_{m}$ e também do posicionamento de cada microfone $\mathbf{p}_{n}$ :

$$
\tau_{m, n}=\frac{\mathbf{a}_{m}^{T} \mathbf{p}_{n}}{c}=-\frac{\mathbf{u}_{m}^{T} \mathbf{p}_{n}}{c}
$$

será o atraso, em relação à origem do sistema de coordenadas adotado, do sinal da $m$-ésima fonte ao chegar no $n$-ésimo microfone. Por superposição, o sinal para o campo todo é a soma dos sinais de todas as fontes pontuais, isto é,

$$
\mathbf{x}(t)=\left[\begin{array}{c}
\sum_{m=0}^{M-1} f_{m}\left(t-\tau_{m, 0}\right) \\
\sum_{m=0}^{M-1} f_{m}\left(t-\tau_{m, 1}\right) \\
\vdots \\
\sum_{m=0}^{M-1} f_{m}\left(t-\tau_{m, N-1}\right)
\end{array}\right] .
$$

Todos os sinais de fontes $f_{m}(t)$ são senóides em uma frequência específica que se queira observar, pois basta implementar processamento de banda estreita, já que o processamento de banda larga é obtido com sucessivas aplicações do processamento de banda estreita para cada componente de frequência do sinal. Depois de gerado $\mathbf{x}(t)$, ruído AWGN é aplicado a cada componente.

A equação (8.3) é aplicada fazendo com que o simulador tenha uma versão amostrada $\mathbf{x}_{n}(t)$ dos sinais $\mathbf{x}(t)$. Por fim, bastaria aplicar a transformada discreta de Fourier (DFT) na forma de FFT para obter $\tilde{\mathbf{x}}(\omega)$. No entanto, o simulador, depois de obter $\mathbf{x}(t)$, utiliza o modelo de snapshots para estimar $\tilde{\mathbf{x}}\left(\omega_{k}\right)$, que consiste em aplicar a FFT em cada snapshots separadamente.

Os algoritmos eficientes também necessitam de uma amostragem separável das direções do campo, porém essa amostragem deve ser separável no espaço $U=[-1,1]^{2} \mathrm{e}$ não na semi-esfera superior. O simulador utiliza amostragem uniforme para satisfazer as condições do método que utiliza NFFT e, assim, poder contemplar todos os algoritmos eficientes. Um exemplo da amostragem gerada pelo simulador de um campo $M=8 \times 8=64$ é mostrado na Figura 27.

Outra possibilidade é gerar $\tilde{\mathbf{x}}\left(\omega_{k}\right)$ indiretamente. Para isto, considere a equação (4.12) sem o ruído:

$$
\tilde{\mathbf{x}}\left(\omega_{k}\right)=\mathbf{V}\left(\omega_{k}\right) \tilde{\mathbf{f}}\left(\omega_{k}\right) .
$$

Então, basta gerar uma estimativa de $\mathbf{f}\left(\omega_{k}\right)$ denotada $\tilde{\mathbf{f}}\left(\omega_{k}\right)$ a partir dos cossenos criados pelo algoritmo utilizando a transformada discreta de Fourier e multiplicar pela matriz diretora do arranjo $\mathbf{V}\left(\omega_{k}\right)$. 


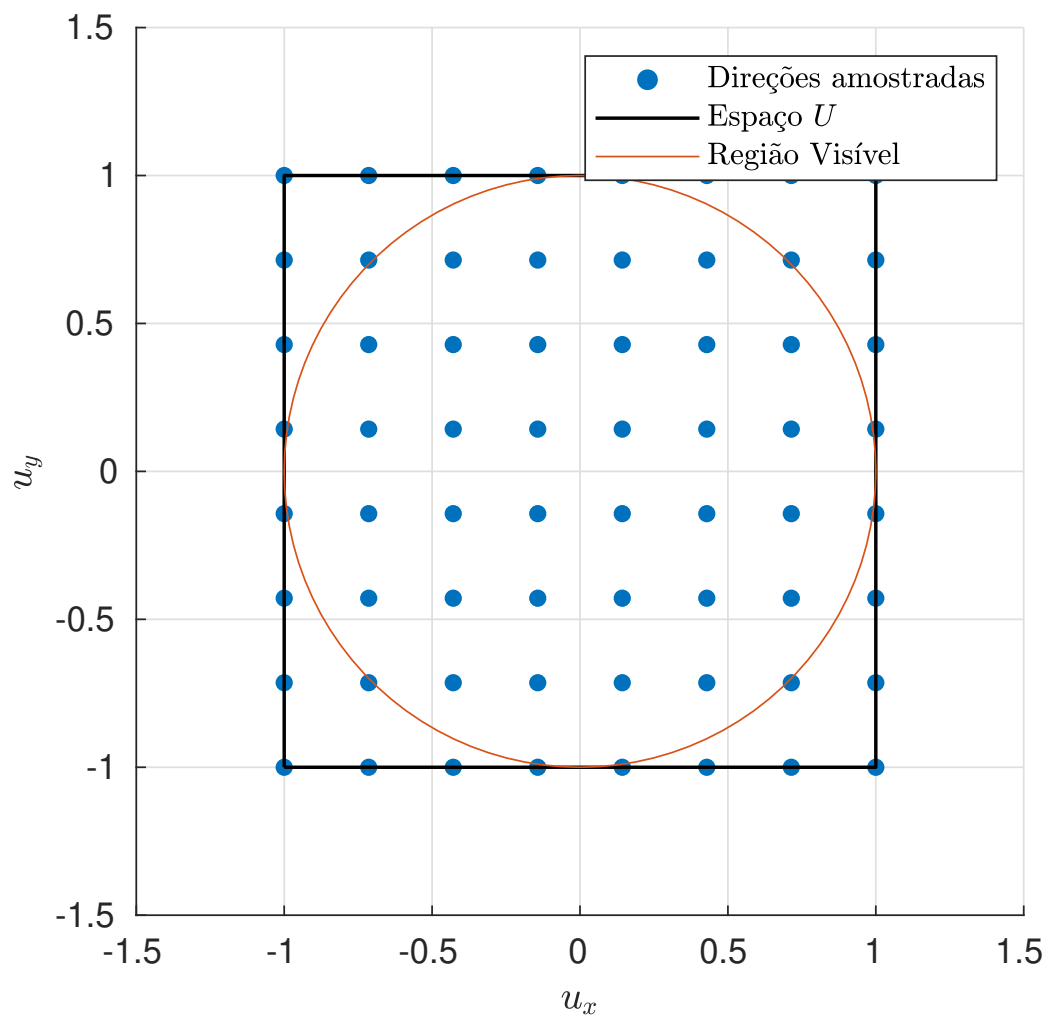

Figura 27 - Amostragem das direções de visada para $M_{x}=M_{y}=8$.

Uma das fontes de erro é a resolução em frequência da DFT. Sabemos que a DFT produz um vetor de $K$ componentes para uma entrada de $K$ amostras e só possui conteúdo de frequência até metade da frequência de amostragem $f_{s}$. Sabe-se também que o uso da DFT implica numa resolução em frequência $d f=f_{s} / K$ devido à amostragem espectral intrínseca à transformada [10,78]. Isto significa que, para um determinado valor de $\omega_{k} \mathrm{e}$ dependendo de $K$, não existe uma amostra em frequência de $\tilde{\mathbf{x}}$ na frequência $\omega_{k}$, mas sim numa frequência próxima.

Como exemplo, imagine que tomemos a DFT de um sinal senoidal de frequência $f=8000 \mathrm{~Hz}$ com $K=100$ amostras e $f_{s}=48000 \mathrm{~Hz}$, teremos uma resolução de frequência $d f=480 \mathrm{~Hz}$. Se quisermos observar ${ }^{1} f_{k}=8000 \mathrm{~Hz}$ teremos que observar a frequência mais próxima fazendo $\omega_{k}=8160 \mathrm{~Hz}$, pois o espectro não está amostrado no valor escolhido com esta resolução de frequência.

\subsection{Modelo de snapshots}

A primeira etapa do processamento é mostrada na Figura 28 e consiste na segmentação dos sinais de cada microfone em quadros de $K$ amostras cada.

Fazendo $\omega_{k}=2 \pi f_{k}$. 


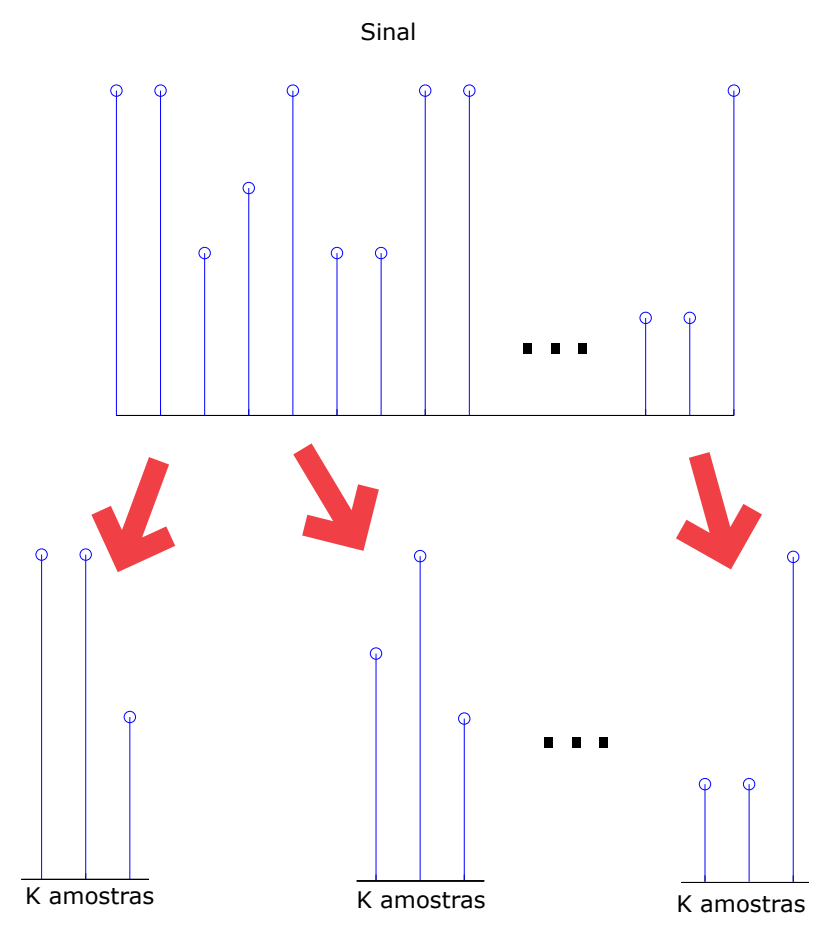

Figura 28 - Método de segmentação dos sinais de entrada de cada microfone em quadros de $K$ amostras cada.

Como estamos interessados em obter um vetor $\mathbf{x}\left(\omega_{k}\right)$ com uma componente de frequência de cada snapshot, o simulador já salva as FFTs dos quadros de $K$ amostras ao invés de salvar o quadro no domínio do tempo.

O simulador pede ao usuário que entre a frequência de observação $\omega_{k}$. A DFT (calculada na forma de FFT) possui uma certa resolução em frequência, fazendo com que a frequência de observação escolhida provavelmente não faça parte da amostragem em frequência. Aqui é feita uma aproximação escolhendo a frequência mais próxima. É possível remediar este erro com zero padding. No entanto, o simulador é capaz de trabalhar com qualquer valor de $K$, sendo a aproximação melhor quanto maior for $K$. A escolha de $K$ ótimo para uma determinada aplicação envolve os conceitos definidos no capítulo 3.

Com a aproximação da frequência $\tilde{\omega}_{k}$ calculada pelo simulador, um vetor $\tilde{\mathbf{x}}_{\ell}\left(\tilde{\omega}_{k}\right)$ é tomado para cada $\ell$-ésimo snapshot. Este vetor possui $N$ componentes, isto é, uma componente correspondendo a cada microfone. Por fim, a matriz espectral $\mathbf{S}$ é estimada utilizando exatamente a equação

$$
\tilde{\mathbf{S}}\left(\omega_{k}\right)=\frac{1}{L} \sum_{\ell=0}^{L-1} \tilde{\mathbf{x}}_{\ell}\left(\omega_{k}\right) \tilde{\mathbf{x}}_{\ell}^{H}\left(\omega_{k}\right)
$$

A matriz espectral possui dimensões $N \times N$. 


\subsection{Geração das matrizes V e $\mathbf{A}$}

A matriz diretora do arranjo $\mathbf{V}$ é gerada iterando a equação do vetor diretor (4.8) repetida a seguir

$$
\mathbf{v}_{\mathbf{u}}(\omega, \mathbf{u})=\left[\begin{array}{c}
e^{-j \omega \mathbf{u}^{T} \mathbf{p}_{0} / c} \\
e^{-j \omega \mathbf{u}^{T} \mathbf{p}_{1} / c} \\
\vdots \\
e^{-j \omega \mathbf{u}^{T} \mathbf{p}_{N-1} / c}
\end{array}\right]
$$

conforme equação (4.9), também repetida aqui:

$$
\mathbf{V}\left(\omega_{k}\right)=\left[\begin{array}{llll}
\mathbf{v}_{\mathbf{u}}\left(\omega_{k}, \mathbf{u}_{0}\right) & \mathbf{v}_{\mathbf{u}}\left(\omega_{k}, \mathbf{u}_{1}\right) & \cdots & \mathbf{v}_{\mathbf{u}}\left(\omega_{k}, \mathbf{u}_{M-1}\right)
\end{array}\right] .
$$

O simulador opera com loops que calculam o vetor diretor para cada direção $\mathbf{u}_{m}$ na forma de vetores coluna e os concatenam em uma única matriz. A matriz $\mathbf{V}$ possui dimensões $N \times M$.

A partir da equação (4.25), vemos que a matriz $\mathbf{A}$ é formada a partir do vetor diretor $\mathbf{v}_{\mathbf{u}}$ do arranjo, ou da matriz $\mathbf{V}$ que contém todos os vetores diretores para todas as direções amostradas, de acordo com a equação

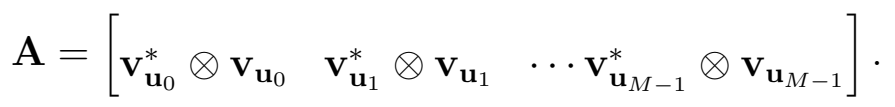

O algoritmo então secciona a matriz $\mathbf{V}$ coluna a coluna e aplica os produtos de Kronecker. A matriz A possui dimensões $N^{2} \times M$.

\subsection{Geração de imagens acústicas}

Nesta seção, alguns exemplos de geração de imagens acústicas são mostrados. O simulador é capaz de trabalhar com algoritmos de beamforming, deconvolução e estimação esparsa.

Considere um campo de teste com $M_{x}=M_{y}=64$ composto por três fontes pontuais de banda estreita na frequência de $6000 \mathrm{~Hz}$ em campo distante em direções arbitrárias. A Figura 29 ilustra a imagem acústica ideal em comparação com as imagens acústicas obtidas pelo simulador utilizando os algoritmos X-DAS, CSM-DAS, DAMAS, DAMAS calculado por PSF e DAMAS utilizando FFT bidimensional. Todas as imagens foram normalizadas em relação ao seu valor máximo.

Considere agora um campo de teste com $M_{x}=M_{y}=32$ composto por duas fontes pontuais de banda estreita na frequência de $8000 \mathrm{~Hz}$ em campo distante em direções arbitrárias. Na Figura 30, pode-se observar as imagens acústicas obtidas pelo simulador utilizando novamente os algoritmos de beamforming e variações de DAMAS. 


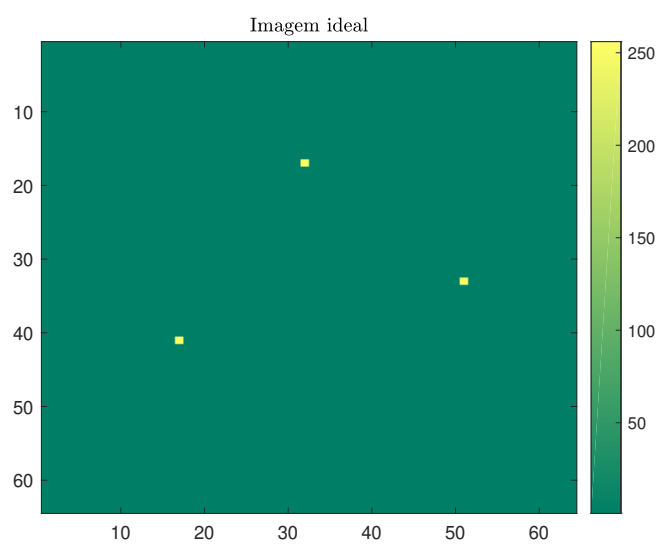

(a)

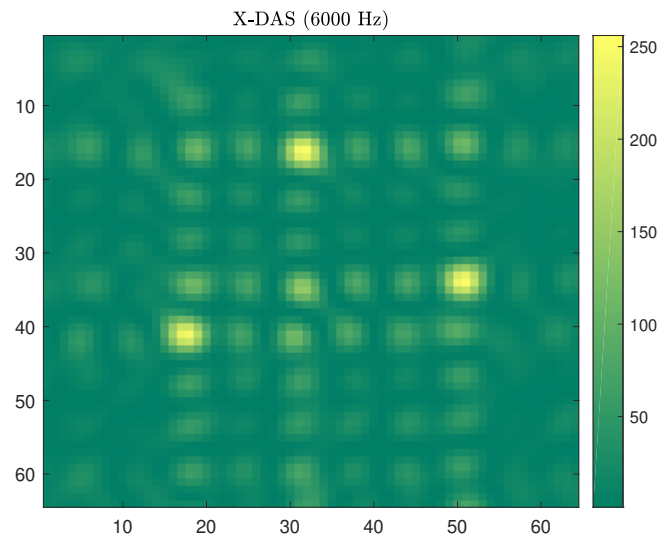

(c)

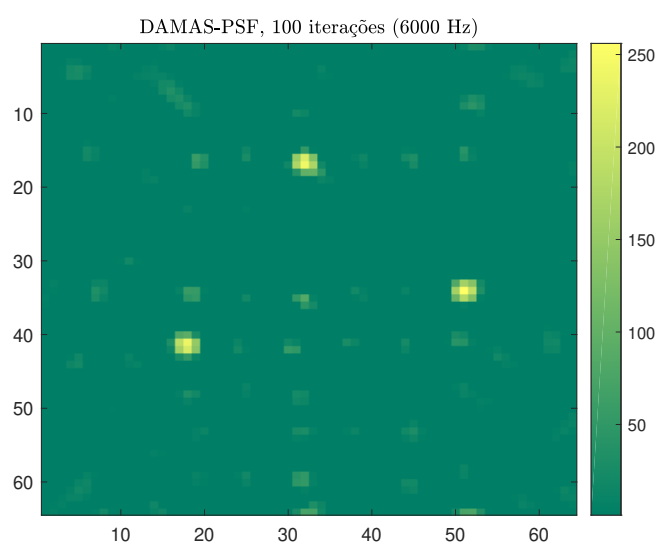

(e)

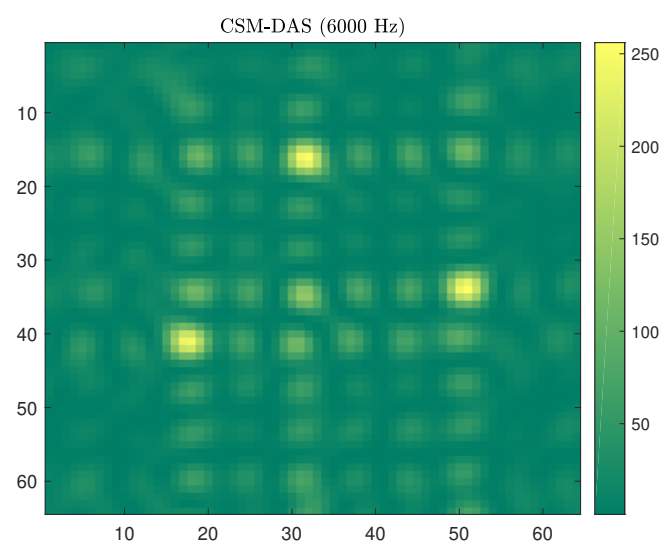

(b)

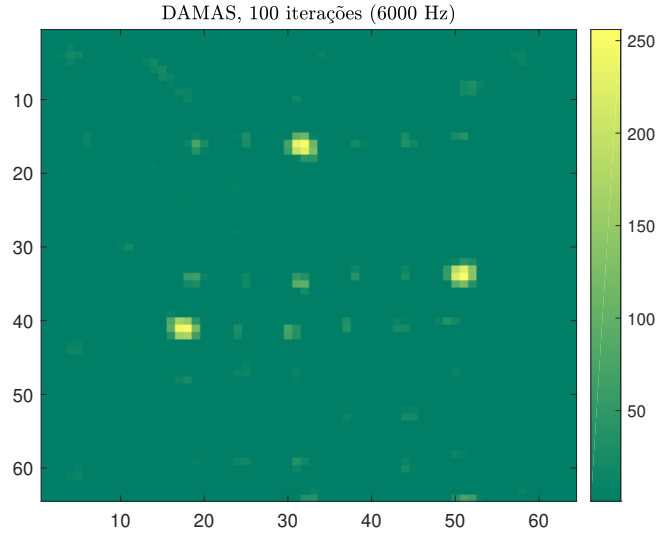

(d)

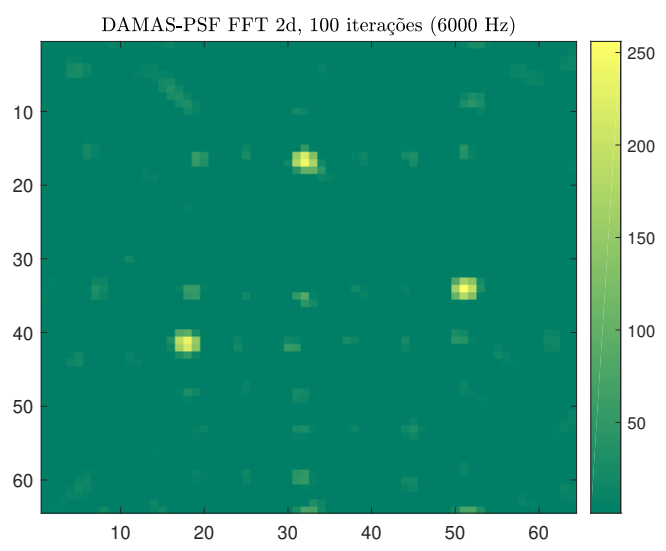

(f)

Figura 29 - Imagens acústicas geradas pelo simulador para um campo de $M=4096$ direções com três fontes pontuais em campo distante na frequência de $6 \mathrm{kHz}$. 


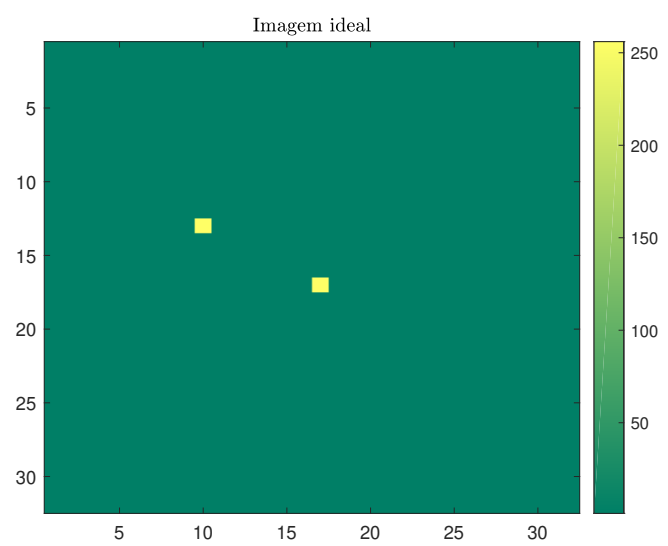

(a)

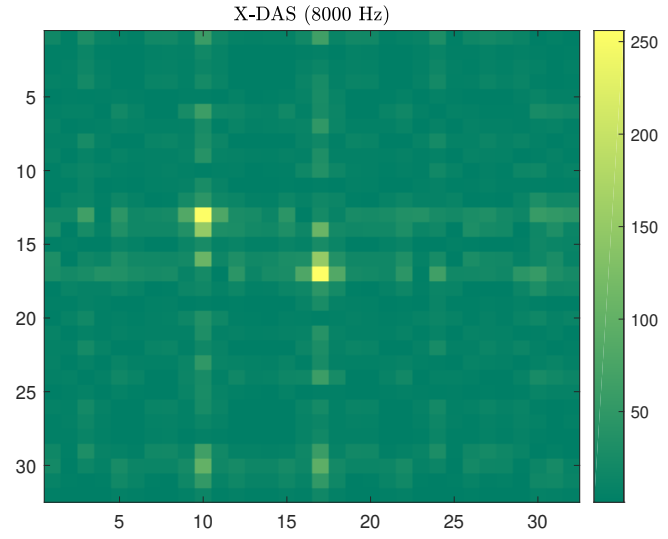

(c)

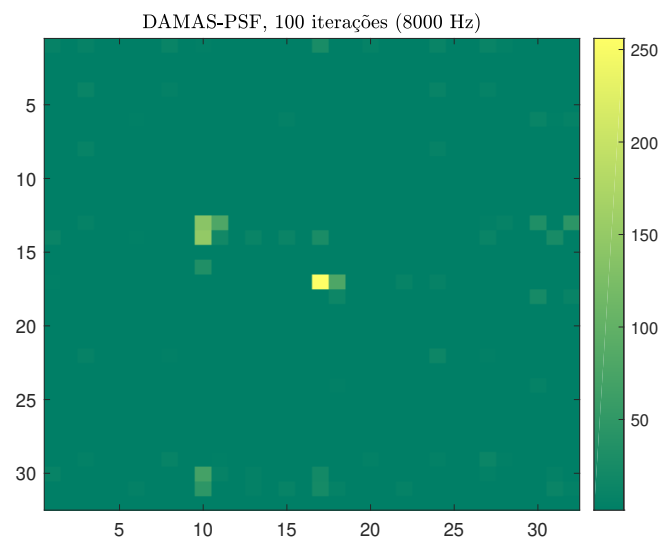

(e)

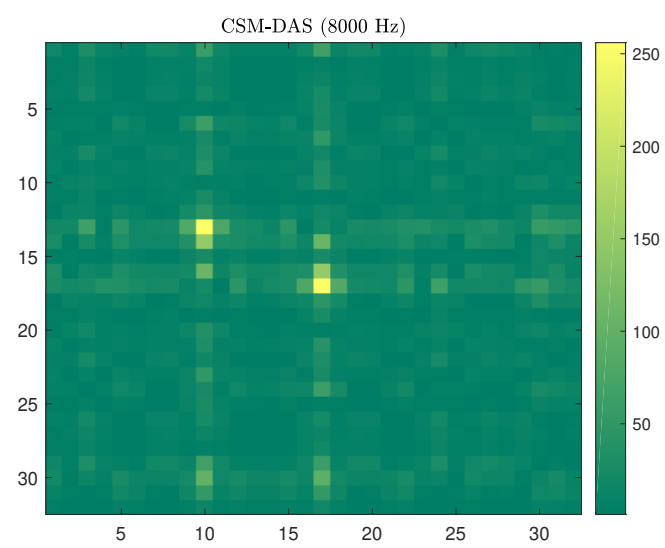

(b)

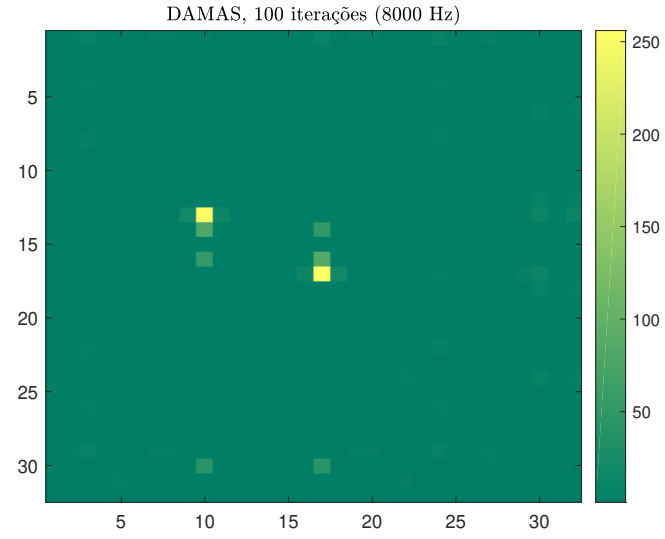

(d)

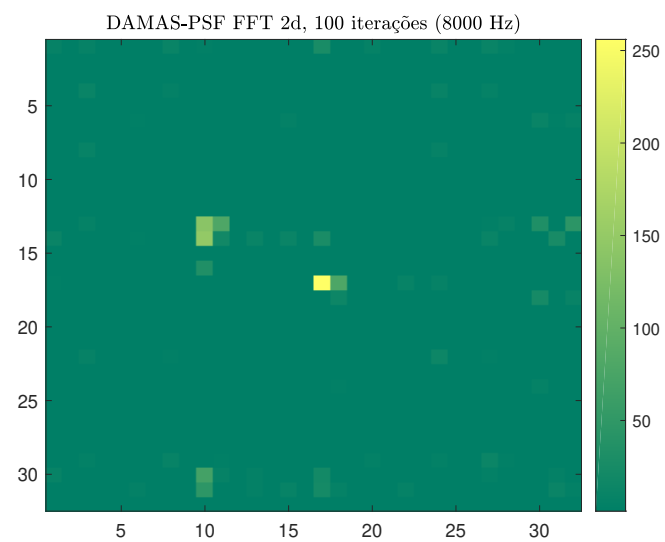

(f)

Figura 30 - Imagens acústicas geradas pelo simulador para um campo de $M=1024$ direções com duas fontes pontuais em campo distante na frequência de $8 \mathrm{kHz}$. 


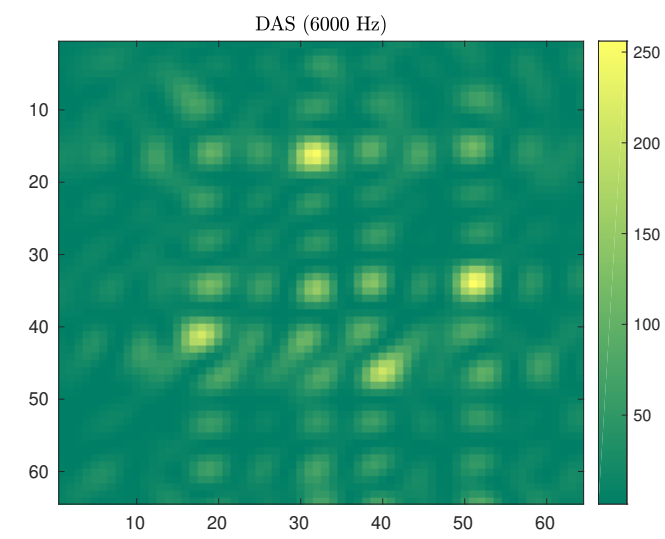

(a)

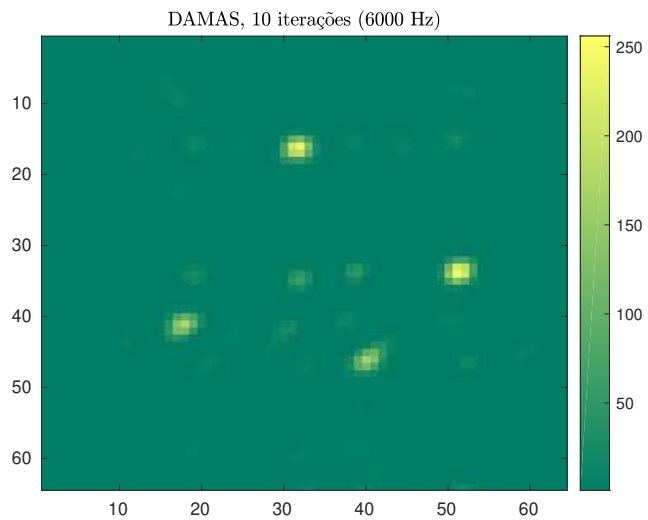

(c)

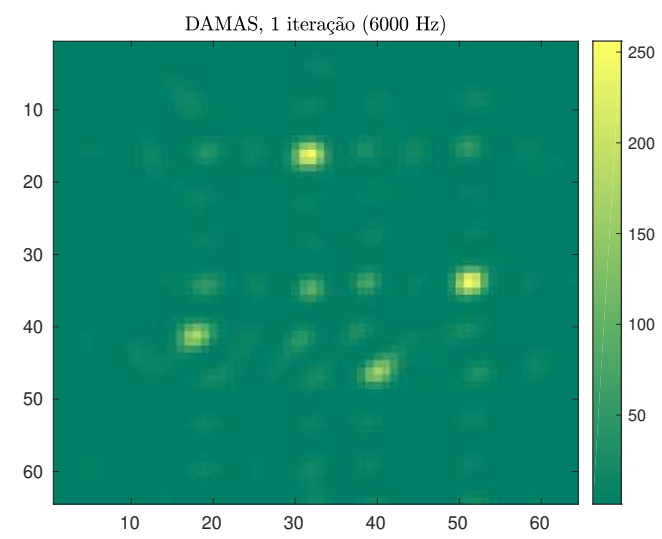

(b)

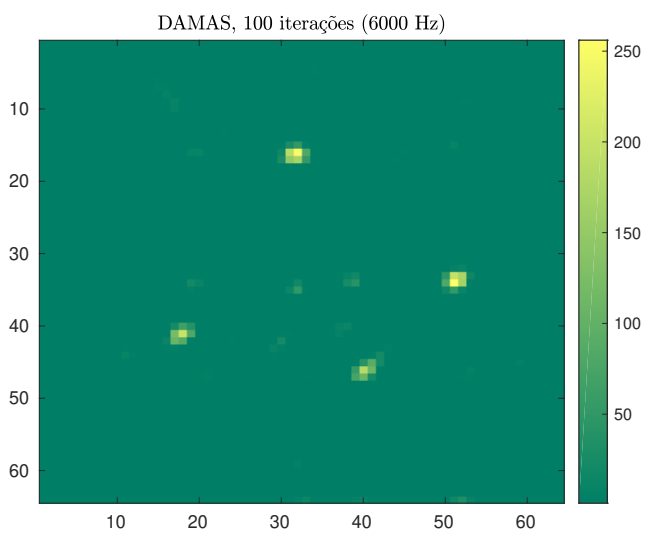

(d)

Figura 31 - Imagens acústicas geradas pelo simulador para um campo de $M=4096$ direções com quatro fontes pontuais em campo distante na frequência de $6 \mathrm{kHz}$ para diferentes números de iterações de DAMAS.

A Figura 31 ilustra a evolução de DAMAS para um caso específico de quatro fontes pontuais em campo distante de frequência igual a $6000 \mathrm{~Hz}$. Já na primeira iteração, o algoritmo é capaz de limpar uma grande quantidade de artefatos da imagem. Na iteração 100, o padrão de fontes possui poucos artefatos intermediários e a aproximação das fontes se encontra menos distribuída que no caso DAS. Os padrões mostrados corroboram o desempenho esperado de DAMAS quando comparado com métodos de beamforming que envolvem a matriz espectral ou o sinal diretamente.

Considere agora um campo de teste com $M_{x}=M_{y}=32 \mathrm{com} 2$ fontes na frequência $11 \mathrm{kHz}$. A Figura 32 mostra o imageamento feito pelo simulador utilizando técnicas de otimização e reconstrução esparsa. Note que os resultados obtidos com técnicas esparsas e de otimização são mais limpos que os obtidos com técnicas de beamforming.

A maioria das imagens dadas como exemplo neste trabalho estão na faixa de $6 \mathrm{kHz}$ a $15 \mathrm{kHz}$, porém nada impede que outras faixas de frequência sejam consideradas. Para fazer o imageamento de outras faixas de frequência, podemos diminuir ou aumentar o 

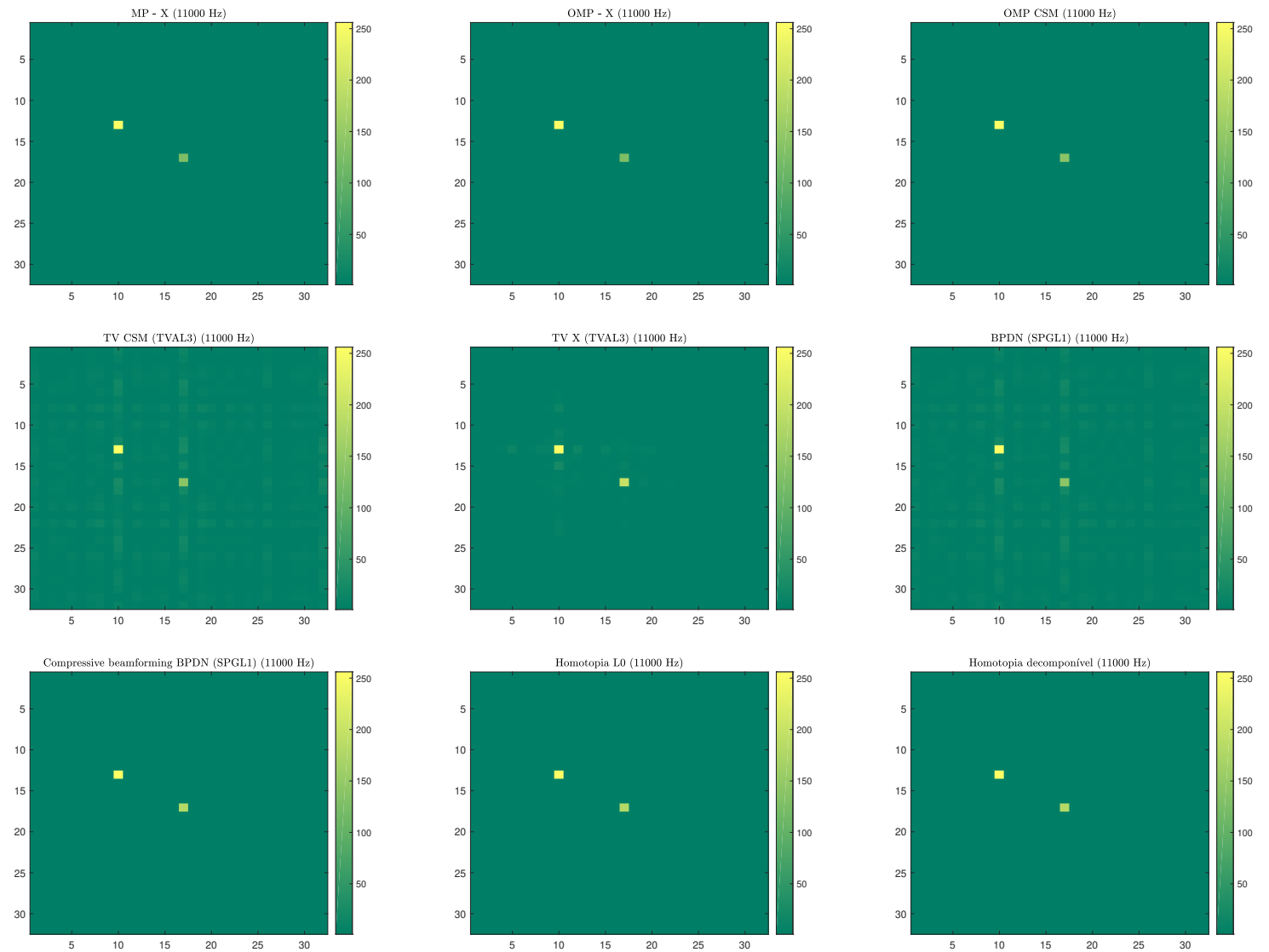

Figura 32 - Imagens acústicas geradas pelo simulador para um campo de dimensões $M_{x}=M_{y}=32 \mathrm{com}$ duas fontes pontuais em campo distante na frequência de $11 \mathrm{kHz}$ para vários algoritmos diferentes de otimização esparsa implementados no sumulador. A posição das fontes é a mesma da Figura 30 porém uma das fontes possui aproximadamete a metade da potência da outra.

tamanho físico do arranjo enquanto mantemos o mesmo tipo de geometria separável e não-redundante mostrada na Figura 8. As bandas de melhor performance na reconstrução de sinais para uma geometria e resolução específicas podem ser determinadas fazendo uma análise análoga à feita por [40].

\subsection{Comparação de qualidade das imagens obtidas}

Uma das maneiras de comparar os resultados é estabelecer métricas de qualidade da imagem obtida.

Neste caso, um campo de teste arbitrário precisa ser escolhido pelo usuário para que o simulador estime as imagens acústicas, ou seja, sempre é possível obter o campo de referência utilizado para estimar as imagens. Portanto pode-se utilizar métricas de comparação que necessitam de uma referência completa ${ }^{2}$, isto é, métodos que compraram

2 Full-reference quality metrics. 
uma imagem "distorcida" obtida por alguma algoritmo com uma "imagem original" sem nenhuma distorção.

A métrica mais simples de qualidade de uma imagem em relação a uma referência é o erro quadrático médio $\left(\mathrm{MSE}^{3}\right)$ definido como

$$
\mathrm{MSE}=\frac{1}{M} \sum_{m=0}^{M-1}\left(\left[\mathbf{y}_{1}\right]_{m, 1}-\left[\mathbf{y}_{2}\right]_{m, 1}\right)^{2},
$$

em que $\mathbf{y}_{1}$ e $\mathbf{y}_{2}$ são versões vetorizadas de imagens acústicas e $M$ é o número de pixels da imagem. O valores do MSE dependem da escala de intensidade da imagem, isto é, um mesmo valor de MSE pode ser muito ruim para uma imagem de 8 bits (com intensidades dos pixels no intervalo [0,255]), mas muito bom para uma imagem de 10 bits (com intensidades dos pixels no intervalo $[0,1023])$.

O PSNR ${ }^{4}$, outra métrica bastante utilizada, resolve o problema de diferentes escalas de intensidade dos pixels normalizando o MSE pelo valor máximo assumido pela imagem

$$
\mathrm{PSNR}=-10 \log _{10} \frac{\mathrm{MSE}}{p^{2}},
$$

em que $p$ é o valor máximo que pode ser assumido por um pixel; para uma imagem de 10 bits, $p=1023$.

Tanto MSE quanto PSNR possuem a vantagem de serem fáceis de calcular, porém são capazes apenas de fazer comparações quando uma mesma imagem de referência é considerada.

A Figura 33a mostra o PSNR para o campo da Figura 29; e a Figura 33b mostra o PSNR para o campo da Figura 30. Os gráficos mostram que OMP se sai melhor nos dois casos. Além disso, pode-se comparar o ganho obtido com a aplicação de DAMAS à imagem obtida com DAS. As descontinuidades que aparecem na 33 podem ser explicadas pela combinação dos seguintes fatores: um ruído de medida foi aplicado em todas as simulações: existem os erros inerentes ao processo de amostragem do sinal; regiões de menor frequência apresentam pior desempenho de reconstrução esparsa para esse tamanho de arranjo [40]; em geral essas descontinuidades acontecem quando o OMP estima um pixel numa direção adjacente ao pixel correto, prejudicando o PSNR porém num caso prático isso seria apenas um erro de DOA. Essas curvas corroboram as vantagem de se utilizar algoritmos de otimização esparsa (representados pelo OMP) resultando em menor erro e mais precisão em aplicações de estimação de direção de chegada. 


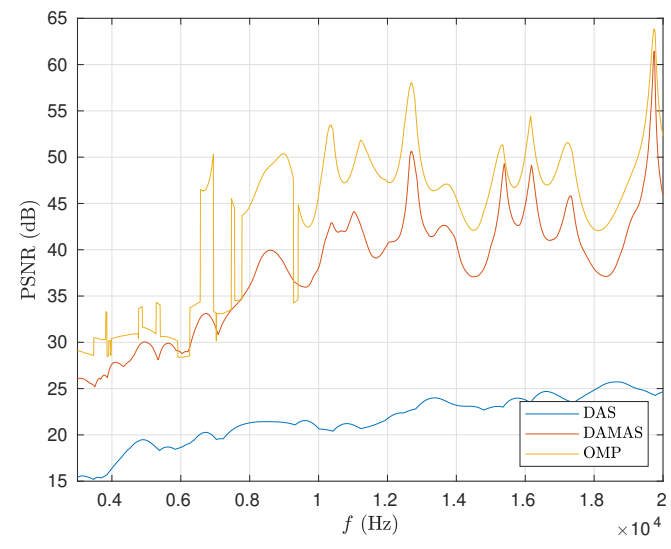

(a)

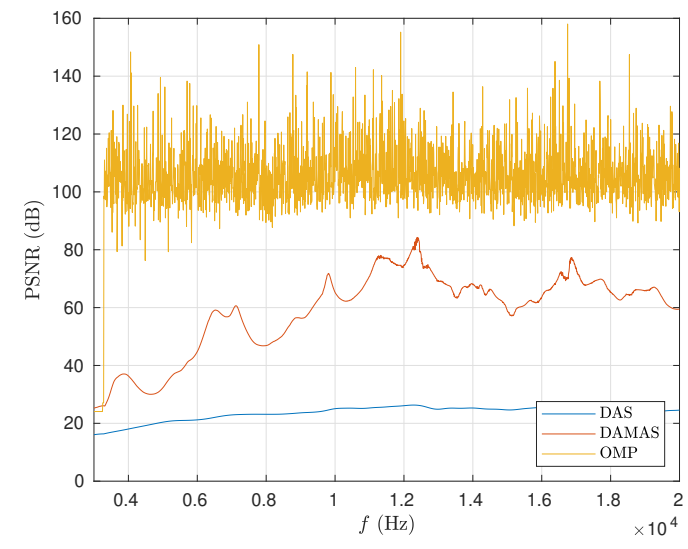

(b)

Figura 33 - PSNR em função da frequência para as distribuições de fontes das Figuras 29 (a) e 30 (b). 



\section{Conclusão}

Este trabalho tratou de algoritmos eficientes para estimação de imagens acústicas que exploram arranjos de geometria separável para reduzir consideravelmente o custo computacional de estimar imagens.

Os arranjos planos separáveis permitem a aplicação da KAT que reduz o custo computacional e os requisitos de memória em várias ordens de magnitude quando comparados ao cálculo direto. A KAT se mostrou muito versátil podendo ser aplicada para algoritmos convencionais de beamforming e deconvolução bem como para técnicas avançadas de otimização.

Depois de descritas as diversas versões da KAT, comparamos os ganhos computacionais obtidos nos métodos convencionais de beamforming e deconvolução mostrando que a KAT possui desempenho superior a outros métodos baseados em transformadas rápidas como NFFT, que ainda são úteis nos casos em que a geometria do arranjo em questão não é separável. Discutimos também o uso de memória dos algoritmos que trabalham com representações explícitas da matriz diretora $\mathbf{V}$ e da matriz $\mathbf{A}$ concluindo que implementações que não dependem dessas matrizes são muito mais vantajosas.

Observando a grande versatilidade da KAT, estendemos o seu uso para alguns algoritmos de otimização esparsa para os quais ela ainda não havia sido aplicada, como regularização TV para estimação direta do sinal e para algoritmos de homotopia. A KAT direta-adjunta em conjunto com a estrutura separável dos arranjos permitiu derivar algoritmos de homotopia independentes da geração de matrizes de dimensão elevada resultando em baixo uso de memória com um custo computacional mais baixo que algoritmos mais avançados como SPGL1 e TVAL3.

Combinamos a KAT com outros métodos rápidos para solução de problemas de mínimos quadrados, como decomposição de Cholesky e decomposição QR, resultando em implementações eficientes do algoritmo guloso OMP.

O estudo dos métodos de compressive beamforming utilizando diretamente o sinal e resolver o mesmo problema de BPDN no equacionamento de ajuste de covariância nos mostrou a diferença computacional entre utilizar métodos diretos e métodos que envolvem a matriz de covariância.

Dois métodos com ideias similares à KAT foram estudados para fins de comparação. O primeiro envolvia o uso da CZT para acelerar cálculos de beamforming com arranjos planos e tridimensionais. Concluímos que a CZT pode ser mais rápida que a KAT em alguns casos, porém é restrita a uma amostragem específica no espaço $U$ e a uma geometria 
mais específica que a separável, de forma que a KAT é mais vantajosa. No entanto, a CZT é uma opção rápida para arranjos tridimensionais, para os quais a KAT não é aplicável.

O segundo envolve soluções eficientes de problemas de mínimos quadrados explorando os produtos de Khatri-Rao, Schur-Hadamard e Kronecker. Como esses três produtos matriciais estão relacionados para o caso específico que ocorre no problema de imagens acústicas para fontes descorrelacionadas, fomos capazes de derivar um novo algoritmo para solução rápida de OMP com equacionamento envolvendo a matriz espectral. O uso desse algoritmo em conjunto com a KAT fornece novas versões eficientes do OMP.

Para fazer as análises, um simulador pra imagens acústicas foi desenvolvido no ambiente Matlab. O simulador é capaz de gerar os sinais dos microfones, calcular as principais matrizes envolvidas, gerar campos de teste e estimar imagens acústicas.

A redução de complexidade computacional conseguida com a KAT em conjunto com os algoritmos que também aproveitam a estrutura separável dos arranjos para reduzir os requisitos de memória, como a nova classe de algoritmos de homotopia, aumentam a viabilidade de aplicações em tempo real de geração de imagens acústicas e permitem o cálculo rápido de imagens de maior dimensão. Uma quantificação mais precisa da viabilidade de aplicações em tempo real precisaria levar em conta a arquitetura específica e os recursos de hardware disponíveis.

Alguns trabalhos futuros que podem ser realizados e não foram discutidos nesse trabalho:

- Estender o conceito de homotopia decomponível para dicionários da forma $\mathbf{V}=$ $\mathbf{V}_{1} \otimes \mathbf{V}_{2} \otimes \cdots \mathbf{V}_{k}$ que não necessariamente ocorrem em aplicações de imagens acústicas;

- Estender o conceito de homotopia decomponível para dicionários da forma $\mathbf{V}=$ $\sum_{k} \mathbf{V}_{1 k} \otimes \mathbf{V}_{2 k}$ que ocorrem na estimação de imagens acústicas em campo próximo $[79]$

- Estender o uso da KAT para outros algoritmos de estimação esparsa que possuem a mesma estrutura de dependência de produtos por $\mathbf{V}$ e $\mathbf{V}^{H}$ ou outras matrizes análogas;

- Aplicações da KAT a problemas de telecomunicações;

- Analisar os novos métodos propostos em aplicações de imagens acústicas. 


\section{Referências}

1 TREES, H. L. V. Optimum Array Processing: Part iv of detection, estimation, and modulation theory. 1. ed. New York: John Wiley \& Sons, 2002.

2 NORDBORG, A.; SEGERHOLM, M. Microphone array measurements of sound radiation from cars. In: Joint Congress CFA/DAGA'04. [S.l.: s.n.], 2004. v. 1, p. 17-18. ISBN 2-9521105-1-4.

3 O'DONOVAN, A.; DURAISWAMI, R.; ZOTKIN, D. Imaging concert hall acoustics using visual and audio cameras. In: 2008 IEEE International Conference on Acoustics, Speech and Signal Processing. [S.l.: s.n.], 2008. p. 5284-5287. ISSN 1520-6149.

4 NASCIMENTO, V. H.; MASIERO, B. S.; RIBEIRO, F. P. Acoustic imaging using the kronecker array transform. In: Signals and Images: Advances and results in speech, estimation, compression, recognition, filtering, and processing. 1. ed. Boca raton: CRC Press, 2016. cap. 6, p. 153-178.

5 BERG, E. van den; FRIEDLANDER, M. P. Probing the pareto frontier for basis pursuit solutions. SIAM Journal on Scientific Computing, v. 31, n. 2, p. 890-912, 2009. Disponível em: <https://doi.org/10.1137/080714488>.

6 MASIERO, B.; NASCIMENTO, V. H. Revisiting the kronecker array transform. IEEE Signal Processing Letters, v. 24, n. 5, p. 525-529, May 2017. ISSN 1070-9908.

7 SANTOS, P. M.; NASCIMENTO, V. H. Estimação de imagens acústicas por orthogonal matching pursuit acelerada por produtos de schur-hadamard e pela transformada de arranjo de kronecker. In: XXXVIII Simpósio Brasileiro de Telecomunicações e Processamento de Sinais. [S.l.: s.n.], 2020. p. 1-6.

8 RIBEIRO, F. P. Arrays de Microfones para Medida de Campos Acústicos. Tese (Doutorado) — Escola Politécnica da Universidade de São Paulo, São Paulo, 2012.

Disponível em: <http://www.teses.usp.br/teses/disponiveis/3/3142/tde-26032012-115753/ pt-br.php>. Acesso em: 8 dez. 2017.

9 JOhnson, D. H.; DudgeOn, D. E. Array Signal Processing: Concepts and techniques. 1. ed. [S.l.]: Prentice Hall, 1993.

10 OpPenheim, A. V.; SCHAFER, R. W. Processamento em tempo discreto de sinais. 3. ed. São Paulo: Pearson Education do Brasil, 2012.

11 LEON-GARCIA, A. Probability, Statistics, and Random Processes for Electrical Engineering. 3. ed. Upper Saddle River: Pearson, 2007.

12 HODGKISS, W. S.; NOLTE, L. W. Covariance between fourier coefficients representing the time waveforms observed from an array of sensors. The Journal of the Acoustic Society of America, v. 59, n. 3, p. 582-590, 1976.

13 BLACHMAN, N. M. On fourier series for gaussian noise. Information and Control, n. 1 , p. 56-63, 1957. 
14 TREES, H. L. V. Detection, Estimation and Modulation Theory: Part ii radar-sonar signal processing and gaussian signals in noise. 1. ed. Danvers: John Wiley \& Sons, 2001.

15 TREES, H. L. V.; BELL, K. L.; TIAN, Z. Detection, Estimation and Modulation Theory: Part 1 - detection, estimation, and filtering theory. 2. ed. Hoboken: John Wiley \& Sons, 2013.

16 RIBEIRO, F. P.; NASCIMENTO, V. H. Fast transforms for acoustic imaging - part i: Theory. IEEE Transactions on Image Processing, v. 20, n. 8, p. 2229-2240, Aug 2011. ISSN 1057-7149.

17 RIBEIRO, F. P.; NASCIMENTO, V. H. Fast transforms for acoustic imaging - part ii: Applications. IEEE Transactions on Image Processing, v. 20, n. 8, p. 2241-2247, Aug 2011. ISSN 1057-7149.

18 NASCIMENTO, V. H.; SILVA, M. C.; MASIERO, B. S. Acoustic image estimation using fast transforms. In: 2016 IEEE Sensor Array and Multichannel Signal Processing Workshop (SAM). [S.l.: s.n.], 2016. p. 1-5.

19 MAYNARD, J. D.; WILLIAMS, E. G.; LEE, Y. Nearfield acoustic holography: I. theory of generalized holography and the development of nah. The Journal of the Acoustical Society of America, v. 78, n. 4, p. 1395-1413, 1985. Disponível em: $<$ https://doi.org/10.1121/1.392911>.

20 PARK, M.; RAFAELY, B. Sound-field analysis by plane-wave decomposition using spherical microphone array. The Journal of the Acoustical Society of America, v. 118, n. 5, p. 3094-3103, 2005. Disponível em: < https://doi.org/10.1121/1.2063108>.

21 RAFAELY, B.; BALMAGES, I.; EGER, L. High-resolution plane-wave decomposition in an auditorium using a dual-radius scanning spherical microphone array. The Journal of the Acoustical Society of America, v. 122, n. 5, p. 2661-2668, 2007. Disponível em: $<$ https://asa.scitation.org/doi/abs/10.1121/1.2783204>.

22 O'DONOVAN, A.; DURAISWAMI, R.; NEUMANN, J. Microphone arrays as generalized cameras for integrated audio visual processing. In: $200^{7}$ IEEE Conference on Computer Vision and Pattern Recognition. [S.l.: s.n.], 2007. p. 1-8. ISSN 1063-6919.

23 RAFAELY, B. Analysis and design of spherical microphone arrays. IEEE Transactions on Speech and Audio Processing, v. 13, n. 1, p. 135-143, Jan 2005. ISSN 1063-6676.

24 HORNE, W. C. et al. Measurements of 26\%-scale 777 airframe noise in the nasa ames 40-by 80 foot wind tunnel. In: 11th AIAA/CEAS Aeroacoustics Conference (26th AIAA Aeroacoustics Conference). [S.l.: s.n.], 2005. p. 1-19.

25 HUMPHREYS, J. W. M.; BROOKS, T. F. Noise spectra and directivity for a scale-model landing gear. In: 13th AIAA/CEAS Aeroacoustics Conference (28th AIAA Aeroacoustics Conference). [S.l.: s.n.], 2007. p. 1-22.

26 LEE, S. S. Phased-array measurement of modern regional aircraft turbofan engine noise. In: 12th AIAA/CEAS Aeroacoustics Conference (27th AIAA Aeroacoustics Conference). [S.l.: s.n.], 2006. p. 1-22. 
27 OERLEMANS, S.; SIJTSMA, P.; LÓPEZ, B. M. Location and quantification of noise sources on a wind turbine. Journal of Sound and Vibration, v. 299, n. 4, p. $869-883$, 2007. ISSN 0022-460X. Disponível em: < http://www.sciencedirect.com/science/article/ $\mathrm{pii} / \mathrm{S} 0022460 \mathrm{X} 06006316>$.

28 WANG, F. Y.; GULSRUD, H. S. W. ans A. Acoustic imaging of aircraft wake vortex dynamics. In: 23rd AIAA Applied Aerodynamics Conference. [S.l.: s.n.], 2005. p. 1-13.

29 BRUSNIAK, L.; UNDERBRINK, J. R.; STOKER, R. W. Acoustic imaging of aircraft noise sources using large aperture phased arrays. In: 12th AIAA/CEAS Aeroacoustics Conference (27th AIAA Aeroacoustics Conference). [S.1.: s.n.], 2006. p. 1-20.

30 HORN, R. A.; JOHnSON, C. R. Topics in Matrix Analysis. [S.1.]: Cambridge University Press, 1991.

31 KHATRI, C. G.; RAO, C. R. Solutions to some functional equations and their applications to characterization of probability distributions. Sankhyā: The Indian Journal of Statistics, Series A (1961-2002), Springer, v. 30, n. 2, p. 167-180, 1968. ISSN 0581572X. Disponível em: <http://www.jstor.org/stable/25049527>.

32 KRIM, H.; VIBERG, M. Two decades of array signal processing research: the parametric approach. IEEE Signal Processing Magazine, v. 13, n. 4, p. 67-94, Jul 1996. ISSN 1053-5888.

33 BLACODON, D. Spectral estimation method for noisy data using a noise reference. Applied Acoustics, v. 72, n. 1, p. 11 - 21, 2011. ISSN 0003-682X. Disponível em: <http://www.sciencedirect.com/science/article/pii/S0003682X10002082>.

34 HUGHETT, P. Linearity and sigma-linearity in discrete-time linear shift-invariant systems. Signal Processing, v. 59, n. 3, p. 329 - 333, 1997. ISSN 0165-1684. Disponível em: <http://www.sciencedirect.com/science/article/pii/S0165168497000595>.

35 EHRENFRIED, K.; KOOP, L. Comparison of iterative deconvolution algorithms for the mapping of acoustic sources. AIA A Journal, v. 45, n. 7, p. $1584-1595,2007$. ISSN 1533-385X. Disponível em: <https://arc.aiaa.org/doi/abs/10.2514/1.26320?journalCode= aiaaj>.

36 DOUGHERTY, R. P. Extensions of damas and benefits and limitations of deconvolution in beamforming. In: 11th AIAA/CEAS Aeroacoustics Conference (26th AIAA Aeroacoustics Conference). [S.l.: s.n.], 2005. p. 1-13.

37 SIJTSMA, P. Clean based on spatial source coherence. In: 13th AIAA/CEAS Aeroacoustics Conference (28th AIAA Aeroacoustics Conference). [S.1.: s.n.], 2007. p. 1-14.

38 WANG, Y. et al. Wideband relax and wideband clean for aeroacoustic imaging. The Journal of the Acoustical Society of America, v. 115, n. 2, p. 757-767, 2004. Disponível em: < https://doi.org/10.1121/1.1639906>.

39 BROOKS, T. F.; HUMPHREYS, W. M. A deconvolution approach for the mapping of acoustic sources (damas) determined from phased microphone arrays. Journal of Sound and Vibration, v. 294, n. 4, p. 856 - 879, 2006. ISSN 0022-460X. Disponível em: <http://www.sciencedirect.com/science/article/pii/S0022460X06000289>. 
40 DAHER, M. A. Arranjos de Microfones para reconstrução de Imagens Acústicas. Dissertação (Mestrado) — Universidade de São Paulo, São Paulo, 2020.

41 HÖGBOM, J. A. Aperture synthesis with a non-regular distribution of interferometer baselines. Astronomy and Astrophysics Supplement, v. 15, p. 417-426, jun. 1974.

42 KEINER, J.; KUNIS, S.; POTTS, D. Using nfft3 - a software library for various nonequispaced fast fourier transforms. ACM Transactions on Mathematical Software, V, n. N, p. 1-23, 2008.

43 LEE, J.-Y.; GREENGARD, L. The type 3 nonuniform fft and its applications. Journal of Computational Physics, v. 206, n. 1, p. 1 - 5, 2005. ISSN 0021-9991.

44 POTTS, D.; STEIDL, G.; TASCHE, M. Fast fourier transforms for nonequispaced data: A tutorial. In:__. Modern Sampling Theory. 1. ed. Boston, MA: Birkhauser, 2001. (Applied and Numerical Harmonic Analysis), cap. 12, p. 247-270.

45 DUTT, A.; ROKHLIN, V. Fast fourier transforms for nonequispaced data. SIAM Journal on Scientific Computing, v. 14, n. 6, p. 1368-1393, 1993.

46 DUAN, H. et al. Spatial resolutions of the broadband nonredundant and minimum redundancy arrays. IEEE Signal Processing Letters, v. 14, n. 11, p. 852-855, 2007.

47 DAHER, M. A. et al. Compressive beamforming and directional sound reconstruction using the kronecker array transform. In: 2019 53rd Asilomar Conference on Signals, Systems, and Computers. [S.1.: s.n.], 2019. p. 133-137.

48 LEV-ARI, H. Efficient solution of linear matrix equations with application to multistatic antenna array processing. Communications in Information and Systems, v. 5, n. 1, p. 123-130, 2005.

49 HENDERSON, H.; SEARLE, S. The vec-permutation matrix, the vec operator and kronecker products: A review. Linear $\&$ Multilinear Algebra - LINEAR MULTILINEAR ALGEBRA, v. 9, p. 271-288, 011981.

50 DUARTE, M. F.; ELDAR, Y. C. Structured compressed sensing: From theory to applications. IEEE Transactions on Signal Processing, v. 59, n. 9, p. 4053-4085, Sep. 2011. ISSN 1053-587X.

51 XenAKI, A.; GERSTOFT, P.; MOSEGAARD, K. Compressive beamforming. The Journal of the Acoustical Society of America, v. 136, p. 260, 072014.

52 DONOHO, D. L.; TSAIG, Y. Fast solution of $\ell_{1}$-norm minimization problems when the solution may be sparse. IEEE Transactions on Information Theory, v. 54, n. 11, p. 4789-4812, 2008.

53 CHEN, S. S.; DONOHO, D. L.; SAUNDERS, M. A. Atomic decomposition by basis pursuit. SIAM Rev., Society for Industrial and Applied Mathematics, USA, v. 43, n. 1, p. 129-159, jan. 2001. ISSN 0036-1445. Disponível em: $<$ https://doi.org/10.1137/S003614450037906X>.

54 TROPP, J. A.; WRIGHT, S. J. Computational methods for sparse solution of linear inverse problems. Proceedings of the IEEE, v. 98, n. 6, p. 948-958, June 2010. ISSN 0018-9219. 
55 MALLAT, S. G.; ZHANG, Z. Matching pursuits with time-frequency dictionaries. IEEE Transactions on Signal Processing, v. 41, n. 12, p. 3397-3415, 1993.

56 BLUMENSATH, T.; DAVIES, M. E. Gradient pursuits. IEEE Transactions on Signal Processing, v. 56, n. 6, p. 2370-2382, 2008.

57 ZAKHAROV, Y. V.; NASCIMENTO, V. Orthogonal matching pursuit with dcd iterations. Electronics Letters, v. 49, n. 4, p. 295-297, Feb 2013. ISSN 0013-5194.

58 CAMPBELL, S. L.; MEYER, C. D. Generalized Inverses of Linear Transformations. 1. ed. Philadelphia, PA: SIAM, 2009. (56). ISBN 978-0-898716-71-9.

59 TROPP, J. A.; GILBERT, A. C. Signal recovery from random measurements via orthogonal matching pursuit. IEEE Transactions on Information Theory, v. 53, n. 12, p. 4655-4666, Dec 2007. ISSN 0018-9448.

60 RUBINSTEIN, R.; ZIBULEVSKY, M.; ELAD, M. Efficient implementation of the k-svd algorithm using batch orthogonal matching pursuit. CS Technion, v. 40, 012008.

61 Schmidt, R. Multiple emitter location and signal parameter estimation. IEEE Transactions on Antennas and Propagation, v. 34, n. 3, p. 276-280, March 1986. ISSN $1558-2221$.

62 Roy, R.; Paulraj, A.; Kailath, T. Esprit-a subspace rotation approach to estimation of parameters of cisoids in noise. IEEE Transactions on Acoustics, Speech, and Signal Processing, v. 34, n. 5, p. 1340-1342, October 1986. ISSN 0096-3518.

63 ROY, R.; KAILATH, T. Esprit-estimation of signal parameters via rotational invariance techniques. IEEE Transactions on Acoustics, Speech, and Signal Processing, v. 37, n. 7, p. 984-995, July 1989. ISSN 0096-3518.

64 GRANT, M.; BOYD, S. CVX: Matlab Software for Disciplined Convex Programming, version 2.1. 2014. <http://cvxr.com/cvx>.

65 GRANT, M.; BOYD, S. Graph implementations for nonsmooth convex programs. In: BLONDEL, V.; BOYD, S.; KIMURA, H. (Ed.). Recent Advances in Learning and Control. [S.l.]: Springer-Verlag Limited, 2008, (Lecture Notes in Control and Information Sciences). p. 95-110. <http://stanford.edu/ boyd/graph_dep.html>.

66 BERG, E. van den; FRIEDLANDER, M. P. SPGL1: A solver for large-scale sparse reconstruction. 2019. Https://friedlander.io/spgl1.

67 YARDIBI, T. et al. Sparsity constrained deconvolution approaches for acoustic source mapping. The Journal of the Acoustical Society of America, v. 123, n. 5, p. 2631-2642, 2008. Disponível em: <https://doi.org/10.1121/1.2896754>.

68 LI, C. An efficient algorithm for total variation regularization with applications to the single pixel camera and compressive sensing. Dissertação (Mestrado) - Rice University, USA, 2010.

69 RUDIN, L. I.; OSHER, S.; FATEMI, E. Nonlinear total variation based noise removal algorithms. Physica D: Nonlinear Phenomena, v. 60, n. 1, p. 259 - 268, 1992. ISSN 0167-2789. Disponível em: <http://www.sciencedirect.com/science/article/pii/ $016727899290242 \mathrm{~F}>$. 
70 DIAMOND, S.; BOYD, S. CVXPY: A Python-embedded modeling language for convex optimization. Journal of Machine Learning Research, v. 17, n. 83, p. 1-5, 2016.

71 AGRAWAL, A. et al. A rewriting system for convex optimization problems. Journal of Control and Decision, v. 5, n. 1, p. 42-60, 2018.

72 ZAKHAROV, Y. V. et al. Low-complexity dcd-based sparse recovery algorithms. IEEE Access, v. 5, p. 12737-12750, 2017. ISSN 2169-3536.

73 ZAKHAROV, Y. V.; TOZER, T. C. Multiplication-free iterative algorithm for ls problem. Electronics Letters, v. 40, n. 9, p. 567-569, 2004.

74 ZAKHAROV, Y. V.; NASCIMENTO, V. H. Dcd-rls adaptive filters with penalties for sparse identification. IEEE Transactions on Signal Processing, v. 61, n. 12, p. 3198-3213, June 2013. ISSN 1053-587X.

75 ODELOWO, B. O. A fast beamforming algorithm for planar/volumetric arrays. In: Conference Record of the Thirty-Ninth Asilomar Conference onSignals, Systems and Computers, 2005. [S.1.: s.n.], 2005. p. 1707-1710. ISSN 1058-6393.

76 MARANDA, B. Efficient digital beamforming in the frequency domain. The Journal of the Acoustical Society of America, v. 86, n. 5, p. 1813-1819, 1989. Disponível em: $<$ https://doi.org/10.1121/1.398614>.

77 JOHNSON, C. R. Closure properties of certain positivity classes of matrices under various algebraic operations. Linear Algebra and its Applications, v. 97, p. 243 - 247, 1987. ISSN 0024-3795. Disponível em: <http://www.sciencedirect.com/science/article/pii/ $0024379587901534>$.

78 LATHI, B. P. Linear Systems and Signals. 2. ed. New York: Oxford, 2004.

79 RIBEIRO, F. P.; NASCIMENTO, V. H. Fast near-field acoustic imaging with separable arrays. In: 2011 IEEE Statistical Signal Processing Workshop (SSP). [S.l.: s.n.], 2011. p. 429-432. 